\title{
UBB+1, an important switch in the onset of Alzheimer's disease
}

Citation for published version (APA):

Gentier, R. G. J. (2015). UBB+1, an important switch in the onset of Alzheimer's disease. [Doctoral Thesis, Maastricht University]. Uitgeverij BOXPress. https://doi.org/10.26481/dis.20151105rg

Document status and date:

Published: 01/01/2015

DOI:

10.26481/dis.20151105rg

Document Version:

Publisher's PDF, also known as Version of record

\section{Please check the document version of this publication:}

- A submitted manuscript is the version of the article upon submission and before peer-review. There can be important differences between the submitted version and the official published version of record.

People interested in the research are advised to contact the author for the final version of the publication, or visit the DOI to the publisher's website.

- The final author version and the galley proof are versions of the publication after peer review.

- The final published version features the final layout of the paper including the volume, issue and page numbers.

Link to publication

\footnotetext{
General rights rights.

- You may freely distribute the URL identifying the publication in the public portal. please follow below link for the End User Agreement:

www.umlib.nl/taverne-license

Take down policy

If you believe that this document breaches copyright please contact us at:

repository@maastrichtuniversity.nl

providing details and we will investigate your claim.
}

Copyright and moral rights for the publications made accessible in the public portal are retained by the authors and/or other copyright owners and it is a condition of accessing publications that users recognise and abide by the legal requirements associated with these

- Users may download and print one copy of any publication from the public portal for the purpose of private study or research.

- You may not further distribute the material or use it for any profit-making activity or commercial gain

If the publication is distributed under the terms of Article $25 \mathrm{fa}$ of the Dutch Copyright Act, indicated by the "Taverne" license above, 
$\mathrm{UBB}^{+1}$, an important switch in the onset of Alzheimer's disease 

$\mathrm{UBB}^{+1}$, an important switch in the onset of Alzheimer's disease Romina Jozefa Guillaume Gentier

Printed by: Uitgeverij BOXPress || Proefschriftmaken.nl Cover by: Niko leronymakis - www.mylittlekaleidoscope.com ISBN: 978-94-6295-370-3

(C) 2015 by Romina Gentier, Maastricht, the Netherlands All rights reserved 



\title{
$\mathrm{UBB}^{+1}$, an important switch in the onset of Alzheimer's disease
}

\author{
PROEFSCHRIFT \\ Ter verkrijging van de graad doctor \\ aan de Universiteit Maastricht \\ op gezag van de Rector Magnificus, Prof. Dr. L.L.G Soete \\ volgens het besluit van het Collega van Decanen, \\ in het openbaar te verdedigen \\ op 5 november 2015 om 16:00 uur
}

door

Romina Jozefa Guillaume Gentier 



\section{Promotores}

Prof. Dr. H.W.M. Steinbusch

Prof. Dr. D.A. Hopkins

\section{Co-promotor}

Dr. F.W. Van Leeuwen

\section{Beoordelingscommissie/Evaluation committee}

Prof.Dr. P.J. Peters (voorzitter/chairman)

Prof. Dr. M.H.V. de Baets

Dr. G. Hoogland

Prof. Dr. C. Korth (Heinrich Heine University, Düsseldorf, Germany)

Prof. Dr. J. B. Schulz (RWTH Aachen University, Aachen, Germany)

The research presented in this thesis was performed at the Department Neurosciences, School for Mental Health and Neuroscience, Maastricht University, Maastricht, The Netherlands.

The publication of this thesis was financially supported by: The Faculty of Health, Medicine and Life Sciences (FHML), Maastricht University (UM), Alzheimer Nederland and Internationale Stichting Alzheimer Onderzoek (ISAO). 



\section{Paranimfen}

Denise Hermes

Marjan Philippens 

Success does not come from recognition by others. It is the fruit of what you have planted with love. When the time of harvest comes, you say to yourself. I did it.

(Paulo Coelho)

Succes komt niet voort uit erkenning door anderen. Het is de vrucht van wat je zelf met liefde geplant hebt. Als het moment van oogsten daar is, kun je tegen jezelf zeggen. Het is me gelukt.

(Paulo Coelho) 



\section{Table of Contents}

CHAPTER 1: General introduction

p. 15

Misframed ubiquitin and lack of protein quality control; an early event in Alzheimer's disease. Aims and outline of this thesis.

\section{CHAPTER 2}

p. 45

Localization of mutant ubiquitin in the brain of a transgenic mouse line with proteasomal inhibition and its validation at specific sites in Alzheimer's disease.

\section{CHAPTER 3}

p. 101

Long-term proteasomal inhibition in transgenic mice by $\mathrm{UBB}^{+1}$ expression results in dysfunction of central respiration control reminiscent of brainstem neuropathology in Alzheimer's patients.

CHAPTER 4

p. 125

Accumulation of basic amino acids at mitochondria dictates the cytotoxicity of aberrant ubiquitin.

CHAPTER 5

p. 159

Mutant ubiquitin decrease amyloid $\beta$ plaque formation in a transgenic mouse model of Alzheimer's disease.

CHAPTER 6

p. 187

Paradoxical effects of mutant ubiquitin and $\mathrm{y}$-secretase during modelled Alzheimer's disease neuropathogenesis.

CHAPTER 7: General discussion

p. 219

Summary

p. 251

Samenvatting

p. 255

Valorization

p. 259 


\section{CHAPTER 1}

\section{General introduction}

Misframed ubiquitin and lack of protein quality control; an early event in Alzheimer's disease.

R.J. Gentier and F.W. van Leeuwen. Frontiers in Molecular Neuroscience.2015;8:1-12 


\section{Abstract}

$A \beta$ plaque formation is a prominent cellular hallmark of Alzheimer's disease (AD). To date, immunization trials in AD patients have not been effective in terms of curing or ameliorating dementia. In addition, $Y$-secretase inhibitor strategies await clinical improvements in AD. These approaches were based upon the idea that autosomal dominant mutations in amyloid precursor protein (APP) and presenilin 1 (PS1) genes are predictive for treatment of all AD patients. However most AD patients are of the sporadic form which partly explains the failures to treat this multifactorial disease. The major risk factor for developing sporadic $A D$ is aging whereas the $\varepsilon 4$ polymorphism is the most prominent genetic risk factor. Other medium-risk factors such as TREM 2 and nine low risk factors from Genome Wide Association Studies (GWAS) were associated with AD. Recently, pooled GWAS studies identified protein ubiquitination as one of the key modulators of AD. In addition, a brain site specific strategy was used to compare the proteomes of AD patients by an Ingenuity Pathway Analysis. This strategy revealed numerous proteins that strongly interact with ubiquitin signaling, and pointing to a dysfunctional ubiquitin proteasome system (UPS) as a causal factor in AD.

We reported that DNA-RNA sequence differences in several genes including ubiquitin do occur in $A D$, the resulting misframed protein of which accumulates in the cellular hallmarks of $A D$. This suggests again a functional link between neurodegeneration of the AD type and loss of protein quality control by the UPS. Progress in this field is discussed and modulating the activity of the UPS opens an attractive avenue of research towards slowing down the development of $A D$ and ameliorating its effects by discovering prime targets for AD therapeutics. 


\section{Alzheimer's disease}

Alzheimer's disease (AD) is a progressive multifactorial neurological disease and the most prevalent form of dementia, accounting for $60-80 \%$ of all cases of dementia (Barnes and Yaffe, 2011). Many reviews have been written about this complex neurodegenerative disease e.g., (Selkoe et al., 2012). The sporadic (SAD) and familial (FAD) forms are the two major types of $A D$ which differ in the age and cause of onset (Figure 1) (van Leeuwen et al., 2000). SAD is heterogeneous with risk factors for developing $A D$ including aging, cognitive inactivity, depression, oxidative stress, brain injury,epigenetic factors and mild cognitive impairment $(\mathrm{MCl})$. Cardiovascular factors (cholesterol status, diabetes, midlife hypertension, obesity, physical inactivy and smoking) as well as genetic risk or dubbed timing factors (apolipopprotein E4 polymorphism; Apo\&4) are also associated with the development of SAD (Querfurth and LaFerla, 2010; Thies, 2012). Other medium-risk factors such as a rare missense mutation ( $\mathrm{R} 47 \mathrm{H}$ subsitiution) in the gene encoding the triggering receptor expressed on myeloid cells 2 (TREM2), which has in a normal situation an antiinflammatory role in the brain. This mutation causes an increased risk for $A D$ through aberrant inflammatory processes (Jonsson et al., 2013). An additional nine low risk factors detected via Genome Wide Association Studies (GWAS) were reported to contribute as well to AD (Holton et al., 2013). Genetic factors associated with AD consists of mutations in the genes encoding for the amyloid precursor protein (APP; chromosome 21), presenilin 1 (PS1; chromosome 14) and presenilin 2 (PS2; chromosome 1). 


\section{Percentages of Alzheimer's disease}
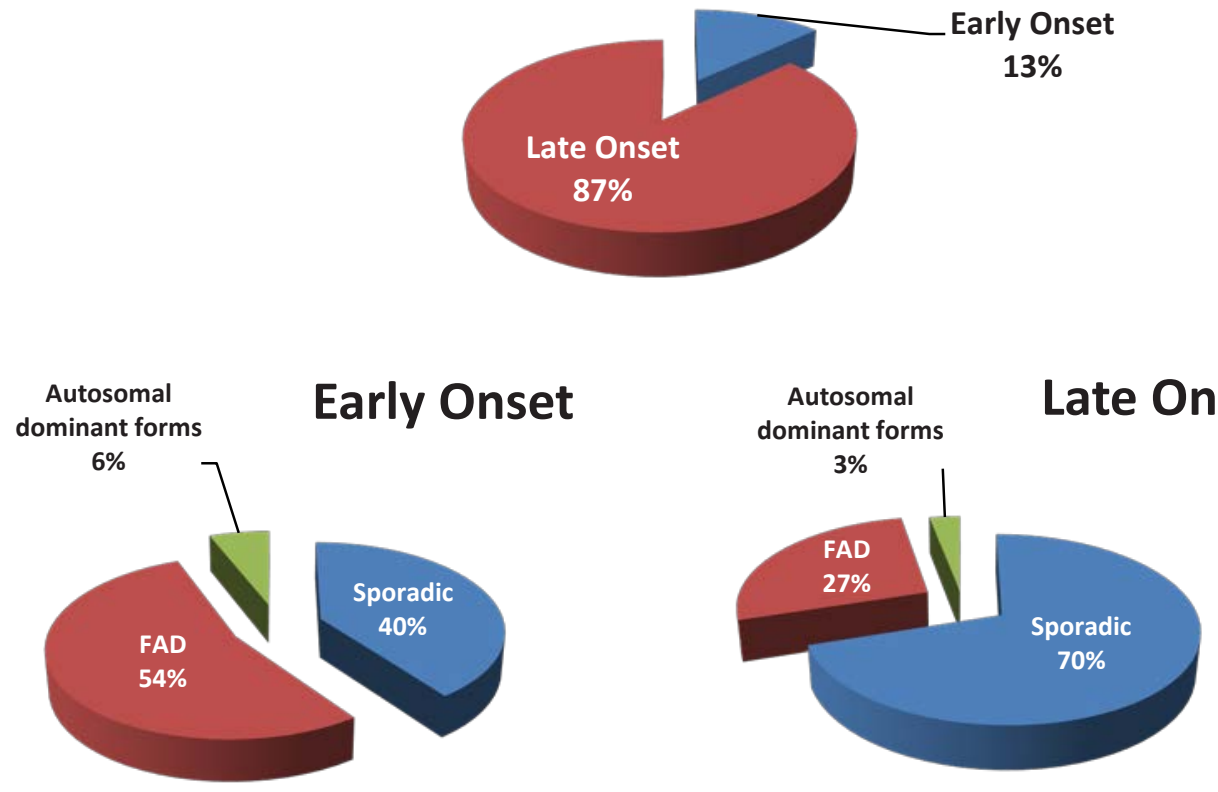

Figure 1: Early (<65 years; EOAD) and late onset (>65 years; $L O A D)$ forms of $A D$ subdivided in familial, sporadic and autosomal dominant forms of $A D$. "Familial" means that $A D$ was observed in relatives of the first degree. In familial EOAD, the majority (54\%) is not yet linked to a chromosome, whereas $6 \%$ is inherited in an autosomal dominant way and linked to chromosome 14 (PS1), chromosome 1 (PS2) and chromosome 21 (APP) (Harvey et al., 2003;Mercy et al., 2008). In familial LOAD, the majority is not yet linked to a chromosome, whereas a minority is inherited in an autosomal dominant way. A subset has been linked to chromosome 12 (Pericak-Vance et al., 1997).

A large number of pathogenic mechanisms in AD have been identified (Jellinger, 2009) including proteasomal dysfunction, oxidative stress, mitochondrial dysfunction, fragmentation of Golgi complex, cellular/axonal transport disruption, mutation of molecular chaperones, dysfunctional neurotrophins, neuroinflammatory processes and more recently seeding (Jucker and Christen, 2013). All these complex mechanisms are interconnected 
resulting in cell dysfunction, neuron shrinkage and cell death such as in parts of the hippocampal complex and locus coeruleus (Mann et al., 1980;West et al., 1994; Heneka et al., 2006). It needs to be noted that neuron loss is not a widespread phenomenon in AD (Regeur et al., 1994) and that cell shrinkage, resulting in weight loss of the brain, erroneously suggested neuron loss, is occurring (Hoogendijk et al., 1995; Lee et al., 2015). The present thesis focusses on 2 mechanisms mentioned above; proteasomal dysfunction by a mutant form of ubiquitin $B\left(U_{B B}^{+1}\right)$ in combination with oxidative stress, both contributing to disease-specific inclusions (van Leeuwen et al., 1998;Dennissen et al., 2012). These aspects are discussed below and in the general discussion in detail.

The neuropathogenesis of $A D$ includes so called negative and positive lesions. Negative lesions describe the loss of cholinergic neurons in the brain, whereas positive lesions are the accumulation of abnormal/misfolded proteins known as deposits (Serrano-Pozo et al., 2011). This accumulation of putative toxic protein species in the brain of the patients is one common hallmark of many neurodegenerative diseases, such as AD and, as well, Parkinson's (PD) and Huntington's (HD) diseases (Fischer et al., 2003). These three diseases belong to a group sharing the common feature of the accumulation of insoluble protein deposits in neurons and are now designated "conformational diseases" coined as such (Lomas and Carrell, 2002). AD is associated with progressive accumulation of two hallmarks, namely extracellular amyloid $\beta(A \beta)$ plaques and intracellular neurofibrillary tangles (NFTs) (Selkoe, 2001;Duyckaerts et al., 2009) Currently there are no indications of increased $A \beta$ and tau protein production in $A D$ which suggests that the accumulation of these proteins is caused by a lack of cellular clearance (Olsson et al., 2003). The degradation pathway of these proteins remains elusive. Although $A \beta$ and tau do not seem to be substrates of the $26 \mathrm{~S}$ proteasome, both proteins are able to impair functioning of the 26S proteasome (Saido and Leissring, 2012). Further details concerning $A \beta$, tau, and the proteasome are given in the following paragraphs.

\section{The $A \beta$ peptide hypothesis}

The "amyloid hypothesis" is supported by the fact that $A \beta$ plaques are present in AD brains and that mutations in the APP gene are involved in the autosomal dominant inherited form 
of AD (Masters and Selkoe, 2012). APP is a precursor molecule which experience proteolysis to generate $A \beta$ species of different length. The $y$-secretase cleavage is a heterogeneous event, so depending on the site of cleavage by this protease $A \beta$-peptides of different size are generated: $A \beta_{40}$ and $A \beta_{42}$ are the most common ones (Haass et al., 2012). The two additional amino acids in $A \beta_{42}$ compared to $A \beta_{40}$ render $A \beta_{42}$ more hydrophobic thereby making it more susceptible aggregate. Due to a higher rate of insolubility and fibrillization, $A \beta_{42}$ is more abundant than $A \beta_{40}$ in extracellular plaques. In many types of $A D$ the ratio of $A \beta_{42 /} A \beta_{40}$ is increased (Masters and Selkoe, 2012). $A \beta$ peptides oligomerize to fibrils plaques which eventually develop in extracellular $A \beta$ plaques (Duyckaerts et al., 2009). Three major types of $A \beta$ plaques can be distinguished in $A D$ : diffuse amyloid plaques, dense-core plaques and neuritic plaques (NPs) (Serrano-Pozo et al., 2011). Currently, there is still an ongoing debate about which species is exactly more toxic: intracellular oligomers or extracellular plaques? A lot of mutations are found within the $y$-secretase complex (PS1, PS2) which cause excessive production of $A \beta_{42}$. The "amyloid hypothesis" focuses on the endoproteolytic cleavage pathway of APP, a transmembrane protein (Glenner and Wong, 1984). Excessive $A \beta$ accumulation is the initiating event in the pathogenesis of $A D$ according to this hypothesis. Normally, a cascade of endoproteolytic cleavages of APP by $\alpha-, \beta$ - and $y$-secretases results in the production of both non-amyloidogenic (the $\alpha$-secretase pathway) and amyloidogenic ( $\beta$-secretase pathway) peptides (Figure 2). It turned out that the formation of $A \beta$ plaques in a transgenic ( $\mathrm{tg}$ ) model of $\mathrm{AD}$ can be modulated by $\mathrm{UBB}^{+1}$ expression via $\mathrm{Y}$-secretase and of this multimeric complex at least the presenilin expression (Chapters 5 and 6 ). 


\section{Non-amyloidogenic pathway}

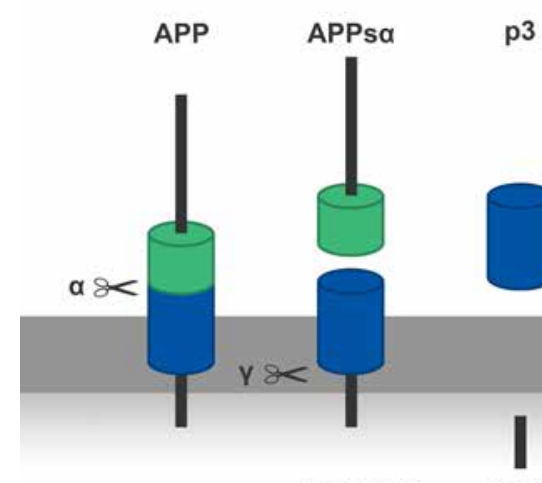

aAPP CTF AICD

\section{Amyloidogenic pathway}

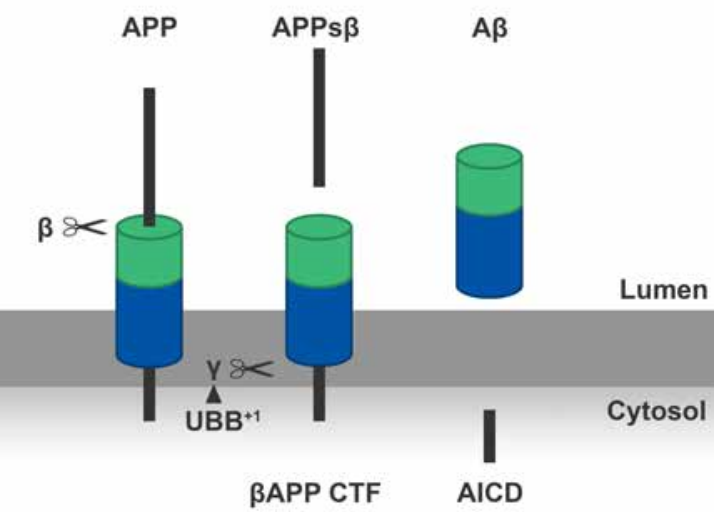

Figure 2: Left panel: $\alpha$-secretase ( $\alpha$ ) cleaves the APP molecules inside the $A \beta$ sequence in a nonamyloidogenic manner, creating a soluble N-terminal part of APP (APPs $\alpha$ ) and a C-terminal part ( $\alpha A P P$ CTF) which is anchored in the membrane. Subsequently the $\gamma$-secretase $(\gamma)$ cleaves the $C$ terminal part in a p3 peptide and an APP intracellular domain (AICD). Right panel: $\beta$-secretase ( $\beta$ ) cleaves the $A P P$ at the $N$-terminus of the $A \beta$-sequence in an amyloidogenic manner, generating an $N$-terminal fragment of APP (APPs $\beta$ ) and a C-terminal part ( $\beta A P P C T F)$. Following, the $\gamma$-secretase ( $V$ ) cleaves the $\beta A P P-C T F$ which results in $A \beta$ and $A I C D$. The present thesis showed that $U B B^{+1}$ is able to modulate the formation of $A \beta$ plaques in the tg line via $y$-secretase, this is shown in the right panel by the arrowhead.

Other studies showed genomic duplications in the APP locus in families suffering from EOAD with concurrent cerebral amyloid angiopathy. These data suggests as well that increased expression of APP has an important role in the AD disease aetiology (RoveletLecrux et al., 2006; Sleegers et al., 2006). In addition, the link between Down syndrome (DS) and $A D$ regarding neuropathogenesis and dementia, supports the relevance of $A \beta$ even further, because DS patients have 3 copies of chromosome 21 and APP is located at the same chromosome. In DS, A $\beta$ plaques already appear around 30 years of life (van Leeuwen et al., 2006). Development of dementia in DS patients is the result of overproduction of toxic $A \beta$ species as a direct consequence of the triplication of the APP gene (Weksler et al., 2013). In addition, a coding mutation (A673T) in the APP gene was 
found in an Icelandic population which protects against $A D$ and cognitive decline in the elderly without AD. This A673T mutation results in an approximately $40 \%$ reduction in the formation of amyloidegenic peptides in vitro and provides proof of principle for the hypothesis that reducing the amyloidogenic cleavage of APP may protect against $A D$ (Jonsson et al., 2012). At the present time, evidence is appearing which suggests that $A \beta$ is neither sufficient nor necessary in the development of $A D$ but only related to it. This finding is based on the observation that the amount and distribution of $A \beta$ accumulation do not correlate with the degree of cognitive impairment and that $A \beta$ is present as well in the brains of cognitively normal elderly people (Drachman, 2014). The prevalence of amyloid pathology increased from age 50 to 90 years from 10\% (95\% Cl, 8\%-13\%) to $44 \%$ (95\% $\mathrm{Cl}, 37 \%-51 \%$ ) among participants with normal cognition (Jansen et al., 2015). In addition, $A \beta$ immunization trials in $A D$ patients have been up to now unsuccessful in terms of curing or ameliorating dementia probably because these clinical trials started too late in the disease process and even resulted in meningoencephalitis in $6 \%$ of the cases (Holmes et al., 2008; Lannfelt et al., 2014). A detailed discussion on these trials goes beyond the scope of the present thesis. A limited clearance of pre-existing $A \beta$ plaques was also shown in experimental studies with the same tg $A D$ mouse model as used for the present thesis (Tucker et al., 2008). As mentioned before using this transgenic model we were able to lower the formation of $A \beta$ plaques. This shows again that an earlier start of immunization trials is an option (Reiman et al., 2012). Another strategy to lower the amount of $A \beta$ plaques or signs of dementia is the use of $y$-secretase inhibitors that were unsuccessful so far, because these trials were halted due to worsening of the outcomes (De Strooper, 2014). In the present thesis, in Chapter 6 , it is even suggested that stimulation of $\mathrm{y}$-secretase activity is a better policy for certain forms of AD.

\section{The tau hypothesis}

This so-called "Tau and tangle hypothesis" suggests that hyperphosphorylated tau is the primary causative factor in $A D$ development leading to neurotoxicity. This hypothesis is based on the observation that NFT density and distribution correlates with the clinical stage of the disease (Morris et al., 2011;Braak and Del Tredici, 2013). 
The tau gene (located on chromosome 17) is translated into a protein found predominantly in nerve cells, concentrated in axons in normal brain and the protein has six isoforms. It contains a microtubule-binding domain formed out of three or four repeating regions (tau 3 $\mathrm{R}$ and tau $4 \mathrm{R}$ ) (Goedert et al., 2012). The history of tau has been reviewed but mutations in the tau gene have only been shown for tauopathies related to $A D$ (Mandelkow and Mandelkow, 2012). Tau initiates and stabilizes neuronal microtubules, which are components of the cytoskeleton, by binding tubulin in healthy brain (Iqbal et al., 2009; Kadavath et al., 2015). When tau is hyperphoshorylated, the ability to bind microtubules is reduced. Accumulations of hyperphosphorylated and misfolded tau proteins are observed in affected neurons in AD. Under normal conditions tau is a soluble protein but it becomes insoluble in its hyperphosphorylated state. In contrast to the normal function of tau protein, the aberrant protein causes disruption of microtubules, dysregulated axonal transport, sequestration of normal tau and subsequently promotes self-assembly (Simic et al., 2009). Three kinds of tau aggregates can be differentiated in AD: pretangles and NFTs in the cell body of neurons, neuropil threads (NTs) in the dendrites, and dystrophic neurites associated with NPs in the axons (Duyckaerts et al., 2009). These different forms of tau are detected in the present thesis by using the MC1 antibody, recognizing aberrant tau in pretangles, and $\mathrm{CP} 13$, recognizing phosphorylated tau and appeared to coincide with the appearance of $\mathrm{UBB}^{+1}$ (see Chapters 2-4). How UBB ${ }^{+1}$ is related to aberrant and phosphorylated tau, both spatially and temporally needs to be determined. A common procedure to classify the degree of AD pathology is the so-called Braak staging (stages $\mathrm{I}-\mathrm{VI}$ ) based on topographical and time-dependent distribution of NFTs and NTs (Braak and Braak, 1991). It was generally accepted that the progression of tau pathology follows a consistent pattern of propagation, which initiates in the transentorhinal cortex and eventually affects all the subdivisions of the neocortex (Braak and Braak, 1995). Currently, however, more evidence is available suggesting that tau aggregation starts in other regions of the brain, such as the brainstem, in particular the locus coeruleus (Braak and Del Tredici, 2011). Based up on these data now also tau immunizations have started e.g., (Sankaranarayanan et al., 2015). 


\section{The ubiquitin proteasome system and $\mathrm{UBB}^{+1}$}

The levels of many proteins must be highly regulated both spatially and temporally in order for cellular functions to proceed accurately. The ubiquitin proteasome system (UPS) is one of the major intracellular mechanisms responsible for executing this process severely contributing to homeostasis in eukaryotic cells. Apart from cellular homeostasis, this system plays an essential role in maintaining neuronal functioning, regulation of chromatin structure, DNA repair, transcriptional regulation, cell cycle and cell division, synaptic development, maintaining synaptic connections and numerous other functions (Ciechanover, 2005). The UPS proteolytic pathway does this by the selective ATPdependent degradation of target proteins such as short-lived, truncated or misfolded proteins by tagging these with polyubiquitin chains. In addition to the ubiquitination, other post-transcriptional modifications such as SUMOylation and NEDDylation contribute to protein degradation (Dennissen et al., 2012) and autophagic processes are activated when the UPS capacity is exceeded (Nixon, 2013).

Ubiquitin (UBB) is a highly conserved signaling molecule of 76 amino acids with a molecular mass of $8.5 \mathrm{kDa}$, that acts in the membrane, nucleus, and cytoplasm of all eukaryotic cells (Gregori et al., 1995). The main function of this protein is a post-translational modification by covalent attachment via an isopeptide bond between ubiquitin and a target protein which is called ubiquitination. The addition of a chain of UBB moieties on the target molecule is called polyubiquitination. The C-terminal glycine (G76) of the UBB molecule will attach to an internal lysine $(K)$ of the target protein (Figure 3). After tagging the target protein with a polyubiquitin chain, the protein is subsequently translocated and degraded by a $26 \mathrm{~S}$ proteasome complex in an ATP-dependent manner. 


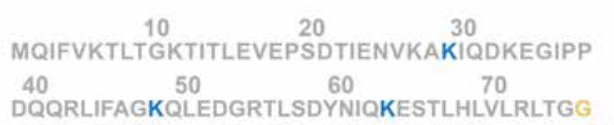

$\Delta \mathrm{GU}$
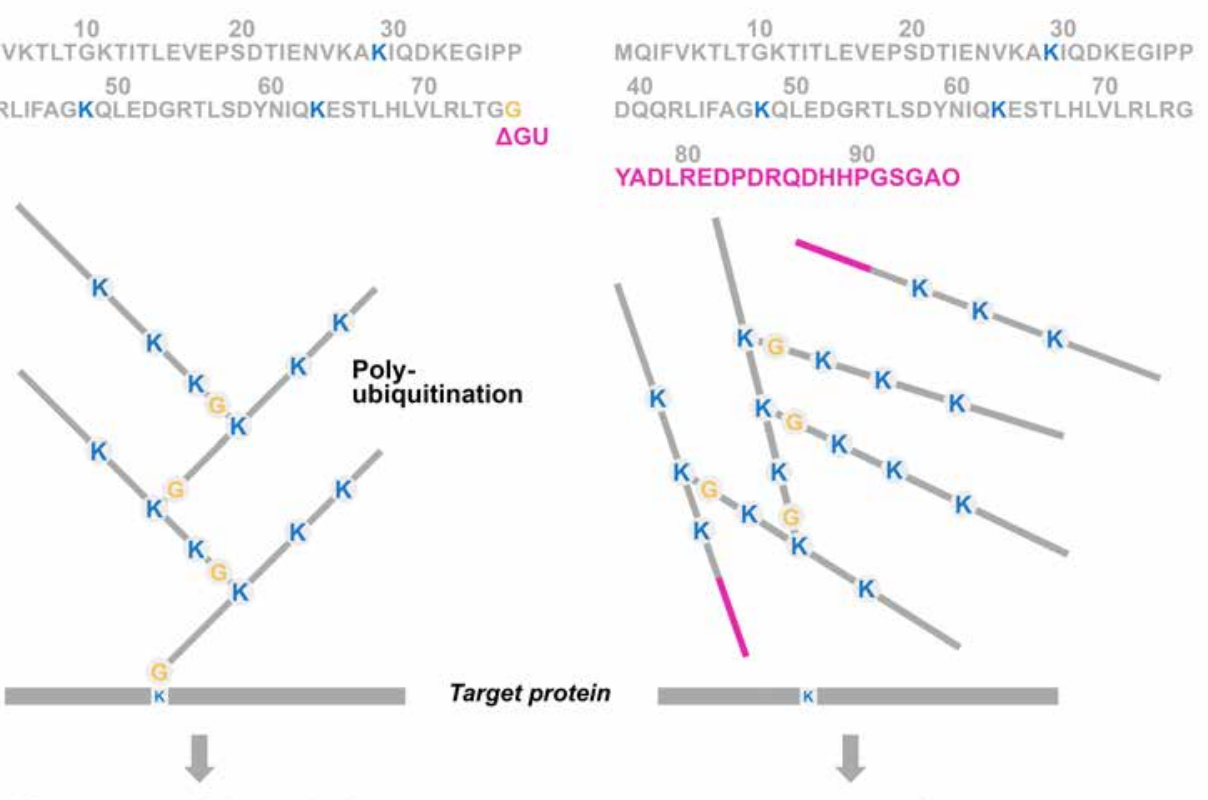

Proteasomal degradation

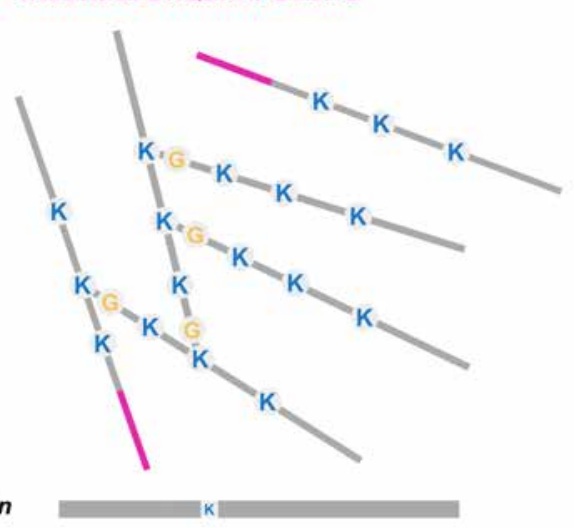

Proteasomal dysfunction

Figure 3: Simplified scheme of the polyubiquitination process of proteasome substrates. In the UPS, a substrate (= a degron, such as a misfolded protein) is (poly)ubiquitinated via a number of enzymatic steps (E1, E2 and E3). The C-terminal glycine (G76) of the UBB molecule will attach to lysine moieties (K) at positions 29, 48 and 63 which are involved in polyubiquitination and degradation. The UBB molecule "on top" of the target protein prone for degradation can be ubiquitinated itself, developing a polyubiquitination chain with at least 4 residues to be efficient for triggering proteasomal degradation (left panel). A GAGAG motif is present at the C-terminus of UBB and a dinucleotide deletion $(\triangle G U)$ occurs adjacent to this motif which results in a 20 amino acids extension (purple bar), called $U_{B B}{ }^{+1}$. This causes the absence of G76, necessary for binding to the target protein, and consequently it is not able to ubiquitinate (Ciechanover, 2015). Interestingly, E3 enzymes are able to form a 'forked' polyubiquitin chain in which 2 ubiquitin chains are linked ot adjacent lysines on a preceding ubiquitin moiety (e.g., K29, K48 and K63). These forked polyubiquitin chains are relatively resistant to degradation by the $26 \mathrm{~S}$ proteasome (Kim et al., 2009).

The conjugation of UBB to the internal lysine residues $(\mathrm{K})$ of the target protein is mediated by a cascade of E1-activating, E2-conjugating, E3-ligating and E4-elongating enzymes, which are stepwise described in Figure 4. UBB is generated from a precursor protein which 
is cleaved by ubiquitin C-terminal hydrolases (UCHs). UBB becomes activated in an ATPdependent manner by the enzyme E1 via a high-energy thiolester bond between the carboxyl group of UBB and the active-site cysteine of E1 enzyme (Pickart, 2001). Subsequently, activated UBB is conjugated to the active site of an E2 ubiquitin-conjugation enzyme by a transthioesterification reaction. Currently, at least 30 different E2 enzymes have been described in the human genome (Bhowmick et al., 2013). Thereafter, UBB is transferred to an internal lysine of the target protein by E3 ubiquitin-protein ligases. There are at least 600 E3 ligases encoded in the human genome (Bhowmick et al., 2013). E3 ligases mediate the ligation between UBB and the target protein resulting in the ubiquitination of the target protein (Ciechanover, 2015). Two different types of E3 enzymes are known: one type, the Homologous to E6-associated protein C-terminus (HECT) binds the E2-enzymes as well as the target protein and serves in this way as an intermediate docking station for UBB. A second type of E3-enzyme is the Real Interesting New Gene (RING) finger containing E3-ligase. In this instance, UBB is transferred directly from the E2-complex to the target protein by the RING-E3 ligase.

The polyubiquitin chain linked with the lysine at position 48 (K48) has to be at least 4 residues long for efficient proteosomal targeting (Thrower et al., 2000). UBB has seven lysine residues indicating that diversity in polyubiquitin chain topology exists in vivo (Peng et al., 2003). As mentioned, the $K 48$ bond involved in proteasome degradation is the most widely known and used topology. However, also different linkages exist like the K63 linkage which mediates non-proteolytic processes (Hadian et al., 2011) or the K11 linkage used for cell-cycle regulation and cell division (Matsumoto et al., 2010). The target protein undergoes several rounds of ubiquitination and in this way a polyubiquitin chain is formed (Ciechanover, 2014). Another, specialized type of E3 enzyme (E4) is necessary for some substrates to be able to become polyubiquitinated to the desired length (Koegl et al., 1999; Hoppe, 2005).

$\mathrm{UBB}^{+1}$ is characterized as the first and only naturally occurring ubiquitin fusion degradation (UFD) substrate, is associated with neurodegeneration and ubiquitinated at K29 and K48 (Lindsten et al., 2002). UBB ${ }^{+1}$ is also ubiquitinated at $\mathrm{K} 63$ which opens the possibility of additional effects of $\mathrm{UBB}^{+1}$ (e.g., kinase activation) (van Tijn et al., 2012). In summary, 
variation in the length of the polyubiquitin chain and the ubiquitin linkage location determine the fate of the ubiquitinated proteins, a process that is most probably more complex than previously assumed (Ciechanover, 2014;2015; Fan et al., 2015).

Subsequently, the polyubiquitin-protein conjugate is degraded by the $26 \mathrm{~S}$ proteasome complex by an ATP-dependent process (Peth et al., 2013) (Figure 5). The abnormal protein is cleaved into short peptide fragments of 6-10 amino acids (Ihara et al., 2012) and the polyubiquitin chain is released. The polyubiquitin chain is degraded by UCHL3 (Dennissen et al., 2011) and further processed by de-ubiquitinating enzymes (DUBs) into monomeric ubiquitin.

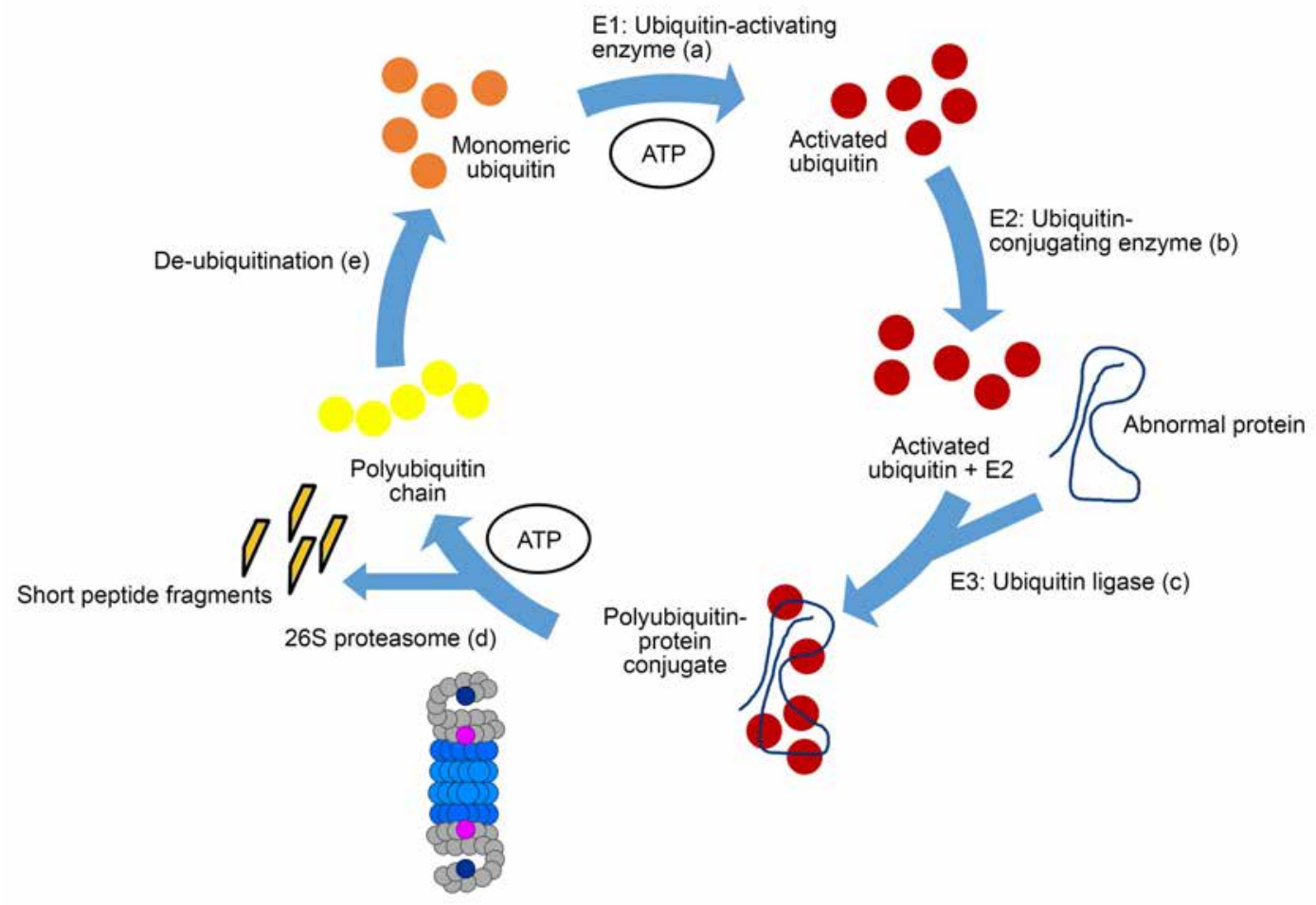

Figure 4: Schematic representation of the ubiquitin proteasome system (UPS). The degradation process by the UPS can be divided into five steps. a) Beginning with monomeric ubiquitin (orange circles). Ubiquitin becomes activated in an ATP-dependent manner by E1. b) Activated ubiquitin (red circles) is conjugated by E2 enzymes. c) Thereafter, UBB is transferred to an internal lysine of the target protein by E3 ligases. Following, activated and conjugated ubiquitin binds to the abnormal 
protein forming the polyubiquitin-protein conjugate. d) Subsequently, the polyubiquitin-protein conjugate is degraded by the 265 proteasome complex (Figure 5) by an ATP-dependent process (Peth et al., 2013). The abnormal protein is cleaved into short peptide fragments (orange pointed bars) and the polyubiquitin chain is released (yellow circles). e) The polyubiquitin chain is split by deubiquitination enzymes into monomeric ubiquitins. For details see (Layfield et al., 2005). Recently, it was shown in yeast cells how transcription errors affect cellular health. In line with our data, these transcription errors represent a molecular mechanism by which cells can acquire disease phenotypes and that it affects proteostasis, particularly in aging cells (Vermulst et al., 2015).

\section{The proteasome}

Polyubiquitinated proteins are translocated to the $26 \mathrm{~S}$ proteasome which is described in detail in Figure 5. The proteasome has to fulfill a whole range of specialized functions 1 ) cleavage of ubiquitin chains linked on the target protein; coined deubiquitination 2) recognition and binding selectively to substrates prone to degradation; 3 ) chaperoning and unfolding of these substrates; 4) opening of the gates on each side of the 20S subunit and 5) driving the substrate into the proteolytic core center in the 20 S cylinder (Wolf and Hilt, 2004). We have performed an extensive immunohistochemical screen of almost all subunits of the proteasome in the hippocampal complex of AD and non-demented controls to see if a differential expression pattern of these subunits could be related to $\mathrm{UBB}^{+1}$ (Zouambia et al., 2008). Therefore the proteasomal complex is now introduced. 


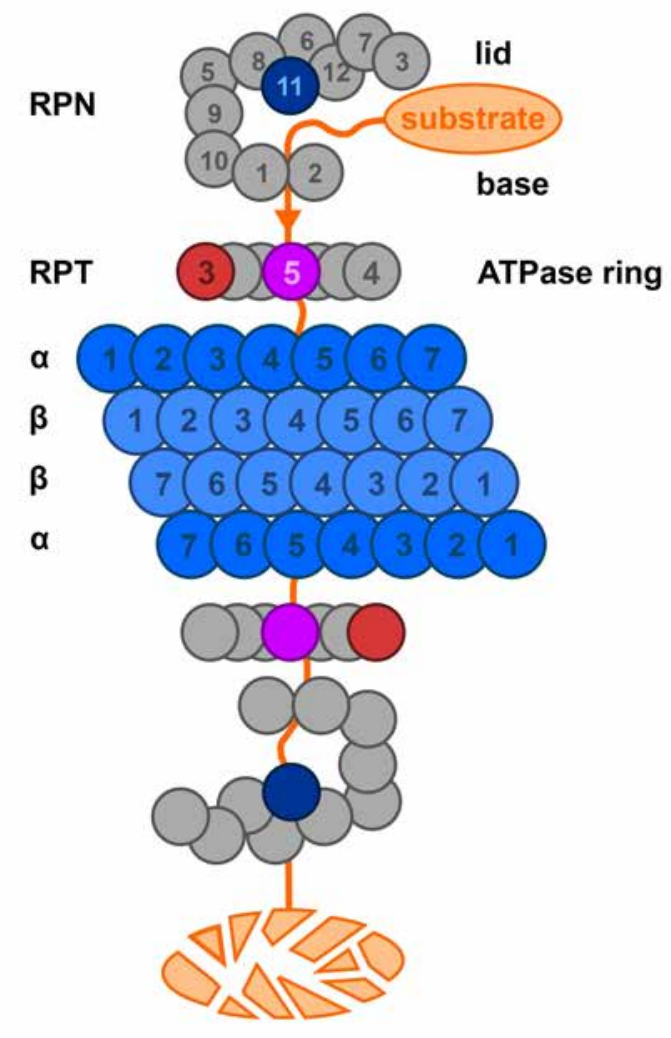

Figure 5: A representation of the 26S proteasome complex based on (Kostova and Wolf, 2003). The lid consists of 8 non-ATP-ase subunits, the base consists of 6 ATP-ase and 3 non-ATP-ase subunits. Please note here that the yeast nomenclature is used. The catalytic core (blue) is a barrel-like structure and consists of 4 stacked heptameric rings, The function of many of the lid, base and ATPase ring subunits such as (Rpt3 (red); Rpt 5 (purple); Rpn 11 (blue)) have been described (for details, see text) (Tsakiri and Trougakos, 2015).

The proteasome is a cylindrical complex of 2.5 MD which consists of two 19S caps and a proteolytic 20S core (Bedford et al., 2010;Ciechanover, 2015). The 20S core complex has a barrel shape comprised of 4 rings and each ring includes 7 subunits. The outer two rings are made up by 7 -subunits facilitating docking of the $19 \mathrm{~S}$ caps. The proteolytic chamber is composed of two inner rings consisting of $\beta$-subunits where $\beta 1, \beta 2$ and $\beta 5$ show proteolytic active sites. The proteolytic activity can be specified in peptidyl-glutamyl-like 
$(\beta 1)$, trypsin-like ( $\beta 2)$ and chymotrypsin-like ( $\beta 5)$ activity. Alternatively, $\beta 1 \mathrm{i}, \beta 2 \mathrm{i}$ and $\beta 5 \mathrm{i}$ can substitute for $\beta 1, \beta 2$ and $\beta 5$ causing a different composition of the $20 \mathrm{~S}$ complex which result in the immunoproteasome. These substitutions can be induced by pro-inflammatory (e.g., interferon- $\gamma$ ) cytokines resulting in immunoproteasomes, responsible for the generation of antigenic peptides presented by the MHC class I complex (Wolf and Hilt, 2004).

The $19 \mathrm{~S}$ activator complexes consist of a base and a lid and are linked to each other via subunit regulatory particle non-ATPase 10 (Rpn10) (S5a in mammals, please note that here the yeast nomenclature is used whereas aliases for the same subunit can be found at sites such as OMIM or Genecards). The base consists of 6 ATPases of the AAA-family of proteins called Regulatory particle triple A proteins 1 to 6 (Rpt1 to Rpt6), 3 additional non-ATPase subunits Rpn1 and Rpn2; and Rpn10/S5a (Zouambia et al., 2008;Ciechanover, 2014). Rpt 5 binds the ubiquitinated substrate. The lid on top of the base is built up out of 8 different subunits (Rpn3, Rpn5 to Rpn9, Rpn 11 and Rpn12). Rpn11 contains a conserved metallo-isopeptidase motif which is necessary for deubiquitination and proteolysis of substrates. It is responsible for the deubiquitination of the targeted substrate after this has been threaded into the $20 \mathrm{~S}$ unit (Tsakiri and Trougakos, 2015). It has been shown in immunohistochemical experiments that the Rpt3 subunit of the base is activated at the transcript level and colocalizes with $\mathrm{UBB}^{+1}$ in NFT of tauopathies including $A D$ but not in synucleinopathies. This upregulation and colocalization of Rpt3 (or subunit $6 \mathrm{~b}$; human alias) with $\mathrm{UBB}^{+1}$ suggests a neuronal reaction to compensate for an increased need to degrade aberrant proteins (Zouambia et al., 2008).

Ultimately, as stated above, the proteasome degrades the substrate into short peptides and, thereby, reusable ubiquitin is released by DUBs. This deubiquitination process is tightly regulated by enzymes called deubiquitinating enzymes (at least 183 DUBS are known) which are partly essential for neuronal functioning. These DUBs can be subdivided into 5 different classes: 1) the ubiquitin C-terminal hydrolases (UCHs); 2) the ubiquitin specific proteases (USPs); 3) the ovarian tumor proteases (OTUs); 4) the Machado-Joseph Disease protein domain proteases (MJDs) and 5) the JAB1/MPN/Mov34 metalloenzymes (JAMMs) (Todi and Paulson, 2011). DUBs are responsible for cleaving ubiquitin 
precursors, rescuing target substrates from degradation, cleaving the ubiquitin chain at the proteasome entrance, controlling epigenetic mechanisms by deubiquitinating histones, and for disassembly of unanchored ubiquitin chains generating single ubiquitin moieties (Komander et al., 2009). Since ubiquitination is a reversible process, DUBs are responsible for maintaining the balance between free UBB and processed UBB (de Vrij et al., 2004;Dennissen et al., 2012). Recently it turned out that extended ubiquitin species like $\mathrm{UBB}^{+1}$ are potent DUB inhibitors leading to accumulation of proteins (Krutauz et al., 2014). This suggests that downregulation of $\mathrm{UBB}^{+1}$ is an attractive strategy to restore neuronal function.

\section{UPS in Alzheimer's disease}

There is compelling evidence that the UPS is impaired in many tauopathies such as AD and is involved in the accumulation of ubiquitinated proteins in AD brains (de Vrij et al., 2004; Ciechanover, 2015). UBB ${ }^{+1}$ is formed during the transcription of the UBB gene by a mechanism that still has to be elucidated, but widespread DNA-RNA sequence differences (RDD) do occur and the mechanism of these changes is becoming clear (van Leeuwen et al., 1998;Gerez et al., 2005; Li et al., 2011;Li et al., 2012;Wang et al., 2014). This process was coined molecular misreading by our group. UBB ${ }^{+1}$ accumulation is found in the neurons of all AD patients and it co-localizes with markers like MC1 and CP13 for NFTs, "kinky and curly" fibres, neuropil threads and dystrophic neurites in plaques (van Leeuwen et al., 1998). UBB ${ }^{+1}$ mRNA was present in all tested brain specimens of non-demented controls, tauo- and synucleinopathies, while the aberrant protein specifically accumulates only all tauopathies investigated so far (e.g., AD) (Fischer et al., 2003) and polyglutaminopathies (de Pril et al., 2004). Interestingly, the accumulation of the $U_{B B}^{+1}$ protein seems not to be initiated by an increase in molecular misreading, as the UBB mRNA concentration measured in cells from temporal cortices of AD patient aged between 54-88 is not increased, nor is it in non-demented controls aged between 38-90 (Gerez et al., 2005). So, question like the driving force of this process, the mechanism of molecular misreading and the difference in protein expression between tauopathies and synucleinopathies remain to be determined. A mechanism to check for errors in mRNA was described by the group of Maquat (Nagy and Maquat, 1998). Apparently $U_{B B}^{+1}$ transcripts escape mRNA 
surveillance as a downstream intron ( $>50$ nucleotides downstream the mutation) is required which lacks in the UBB gene. This process is also called non-sense mediated RNA decay (NMD)(Maquat, 2015).

So $\mathrm{UBB}^{+1}$ accumulates specifically in tauo- and polyglutaminopathies. Apparently, the UPS partly contributes to these phenomena:

a. Ubiquitination of $\mathrm{UBB}^{+1}$ is mediated by the enzymes E2-25K (Ko et al., 2010) and E3 ligases TRIP12 and HUWE1 (Park et al., 2009;Poulsen et al., 2012). E2-25K has an important role in providing a functional interaction between $\mathrm{UBB}^{+1}$ and $A \beta$ toxicity (Ko et al., 2010).

b. $U_{B B}{ }^{+1}$ chains seem to be resistant to disassembly by the DUB isopeptidase $T$, as $\mathrm{UBB}^{+1}$ lacks the C-terminal glycine residue (Lam et al., 2000).

c. Another explanation for $\mathrm{UBB}^{+1}$ accumulation is that it may be due to the inefficient hydrolysis of the C-terminus of $\mathrm{UBB}^{+1}$ by the deubiquitinating enzyme $\mathrm{UCHL} 3$, possibly of the oxidative stress in neurodegenerative diseases (Dennissen et al., 2011).

d. Furthermore it has been shown that $\mathrm{UBB}^{+1}$ is a few amino acids too short to be efficiently degraded by the proteasome (Verhoef et al., 2009).

e. At low levels, the $\mathrm{UBB}^{+1}$ protein will be polyubiquitinated and degraded by the proteasome itself. However, at higher levels it will act as an inhibitor of the proteasome (right panel of Figure 3) (van Tijn et al., 2007). Decreased proteasome activity has been shown in affected brain areas (e.g., hippocampus and cortex) of AD patients (Keller et al., 2000). The UPS exhibited a failure in proteasomal activity in cortical brain areas in AD (Lopez Salon et al., 2000;Zouambia et al., 2008). Interestingly, the accumulation of $A \beta$ in extracellular plaques may have an additional UPS inhibition, an effect shown in a cell-free system (Gregori et al., 1995), in neuronal primary cultures (Lopez Salon et al., 2003) and in several AD tg mouse models (Almeida et al., 2006; Tseng et al., 2008). In addition, paired helical filaments (PHFs) isolated from AD brains are able to inhibit the proteasome via the $20 \mathrm{~S}$ core (Keck et al., 2003). Moreover, the UPS inhibition not only increases the 
accumulation of $\mathrm{UBB}^{+1}$, but it also increases the deposition of hyperphosphorylated tau in NFTs (Morishima-Kawashima et al., 1993; Hol et al., 2005).

f. Earlier it was reported that $U_{B B}^{+1}$ causes neuritic beading, impairment of mitochondrial movements, mitochondrial stress and degeneration of primary neurons (Tan et al., 2007). The underlying mechanism may be due to mitochondrial dysfunction as we showed in a yeast strain that $U_{B B}^{+1}$ disturbs the UPS which causes mitochondrial stress and apoptosis. An unexpected enhancement of basic amino acid synthesis (arginine, ornithine and lysine) at mitochondria was induced by the expression of $\mathrm{UBB}^{+1}$ and this increase was identified as the decisive toxic event. This could be reversed by Cdc48/Vms1-mediated proteolysis which propose this pathway as a novel target for preventing neuronal dysfunction in AD (Chapter 4/ (Braun et al., 2015)).

g. It was shown that $\mathrm{UBB}^{+1}$ expression induces expression of heat-shock proteins (HSPs).This priming of the chaperone system promotes a subsequent resistance to oxidative stress (Hope et al., 2003).

These data suggest that the UPS is a central component in the pathogenesis of AD, a hypothesis recently firmly supported by other studies (Manavalan et al., 2013;IGAP, 2015).

To elucidate the effects of $\mathrm{UBB}^{+1}$ accumulation in the brain, we generated a tg mouse line (line 3413) which shows overexpression of human $\mathrm{UBB}^{+1}$ in neurons of the postnatal brain, with strongest neuronal expression in the forebrain. In in vivo studies, a different behavioral phenotype from wild-type (WT) controls was found in the $3413 \mathrm{tg}$ mice showing deficits in spatial memory retention in the Morris water maze (MWM) as well as deficits in contextdependent fear conditioning (FC) compared with WT mice. The proteome also showed changes compatible with AD (Fischer et al., 2009).

These data together (e.g. cognitive and respiratory dysfunction in combination with a comorbidity such as epilepsy) (Mucke and Selkoe, 2012) highlight the pivotal role of UBB ${ }^{+1}$ in basic mechanisms like homeostasis and breathing (see aim and outline of this thesis). According to Maslow's hierarchy (Maslow, 1943), these basal levels needs to be fulfilled to jump to the next level of satisfaction (see discussion). 


\section{Aim and outline of the thesis}

The aim of the present thesis was to determine the significance of proteasomal dysfunction by the expression of $\mathrm{UBB}^{+1}$, not only in a mouse model for $\mathrm{AD}$ but also in yeast and to further validate the obtained data in postmortem brain tissue of $A D$ donors.

The starting point of this thesis was the use of our tg mouse line 3413 expressing human $\mathrm{UBB}^{+1}$ in the postnatal brain. Chapter 2 presents a comprehensive topographic mapping of $\mathrm{UBB}^{+1}$ in the brain of line 3413 . The extensive $\mathrm{UBB}^{+1}$ expression in these mice led us to compare this with the distribution of $\mathrm{UBB}^{+1}$ and established markers for $\mathrm{AD}$ in the human postmortem brain. The most intense sites of $\mathrm{UBB}^{+1}$ accumulation in the basal ganglia, raphe nuclei. olfactory bulb, inferior colliculus and brainstem show that line 3413 is also useful to study other conformational diseases like HD and Familial encephalopathy with neuroserpin inclusion bodies (FENIB) (Schipanski et al., 2014). This FENIB tg line was crossed with a line 3413 and resulted in an effect on endoplasmic reticulum associated degradation (ERAD). It was also shown in this paper that $\mathrm{UBB}^{+1}$ localization coincides with NFT appearance.

Chapter 3 presents a specific aspect of the $\mathrm{UBB}^{+1}$ mapping study that demonstrates strong $\mathrm{UBB}^{+1}$ immunoreactivity in brainstem regions of tg mice controlling respiration. This has been linked to the changes in spontaneous breathing patterns and an altered response to hypoxic conditions as revealed in a functional analysis in the German Mouse Clinic. The focus in $A D$ research has so far been on the entorhinal cortex, temporal cortex and hippocampus while the brainstem has been under investigated. Very recently, a study appeared showing significant total volume reduction and deformations in the brainstem of $A D$ patients (Lee et al., 2015). To validate our findings, $U_{B B}^{+1}$ expression was examined in the same human $A D$ brainstem regions to possibly link the disproportional contribution of bronchopneumonia as a cause of death in AD patients to proteasomal dysfunction. It is suggested that brainstem dysfunction (e.g., respiration problems) is an early symptom as a result of UPS dysfunction. Indeed our data are consistent with respiratory symptoms also observed in AD patients (see for details (Irmler et al., 2012). This study confirms the power of a complete phenotypic screening of a tg line, such as was performed by the German Mouse Clinic. 
In Chapter 4 a yeast model has been used for exploring the detrimental effects of $\mathrm{UBB}^{+1}$ on cell death mechanisms, in which mitochondria play a pivotal role in the induction of cell death. The link between $\mathrm{UBB}^{+1}$ expression and enhancement of the basic amino acids arginine, ornithine and lysine was shown as a toxic event which can be reversed by the mitochondrial mediated proteolysis by $\mathrm{CdC} 48 / \mathrm{Vms} 1$ proteins. These role of these proteins obtained in yeast studies were validated in human brain tissue by immunohistochemical analyses. The protective mechanism of Vms1 has potentially far-reaching pathophysiological implications.

In Chapter 5 a remarkable decrease in $A \beta$ plaque formation and $A \beta_{42}$ levels was found in a crossbreed between $\mathrm{UBB}^{+1}$ tg mice and the APPPS1 mice carrying the Swedish mutation (K594M/N595L) and human PS1 with a deletion of exon 9 (APPPS1). This was in contrast to what was expected: an increase in $A \beta$ plaque load due to proteasomal inhibition by $\mathrm{UBB}^{+1}$.

In Chapter 6 the molecular mechanisms behind the decrease in $A \beta$ plaque load in an environment of proteasomal stress and the effect on behavioral levels were examined in APPPS1 and the crossbreed APPPS1/UBB ${ }^{+1}$. Contrary to most of the literature on $\mathrm{Y}^{-}$ secretase activity in $A D$, a decreased $Y$-secretase activity, a flawed expression of PS1 and a worsened behavioral phenotype were shown APPPS1 mice. Moreover, in the crossbreed (APPPS1/UBB ${ }^{+1}$ ) an even worse behavioral phenotype was observed with less $A \beta$ plaque load. These data suggest a protective role for $A \beta$ plaques in $A D$. In addition, the unsuccessful search for $\mathrm{y}$-secretase inhibitors (De Strooper, 2014) must take this aspect in to account.

In Chapter 7, the main conclusions of the present thesis are summarized, a valorization paragraph is added and an outlook for follow up experiments is presented. 


\section{References}

Almeida, C.G., Takahashi, R.H., and Gouras, G.K. (2006). Beta-amyloid accumulation impairs multivesicular body sorting by inhibiting the ubiquitin-proteasome system. J Neurosci 26, 4277-4288.

Barnes, D.E., and Yaffe, K. (2011). The projected effect of risk factor reduction on Alzheimer's disease prevalence. Lancet Neurology 10, 819-828.

Bedford, L., Paine, S., Sheppard, P.W., Mayer, R.J., and Roelofs, J. (2010). Assembly, structure, and function of the 26S proteasome. Trends Cell Biol 20, 391-401.

Bhowmick, P., Pancsa, R., Guharoy, M., and Tompa, P. (2013). Functional diversity and structural disorder in the human ubiquitination pathway. PLoS One 8, e65443.

Braak, H., and Braak, E. (1991). Neuropathological stageing of Alzheimer-related changes. Acta Neuropathol 82, 239-259.

Braak, H., and Braak, E. (1995). Staging of Alzheimer's disease-related neurofibrillary changes. Neurobiol Aging 16, 271-278; discussion 278-284.

Braak, H., and Del Tredici, K. (2011). The pathological process underlying Alzheimer's disease in individuals under thirty. Acta Neuropathol 121, 171-181.

Braak, H., and Del Tredici, K. (2013). Evolutional aspects of Alzheimer's disease pathogenesis. $J$ Alzheimers Dis 33 Suppl 1, S155-161.

Braun, R.J., Sommer, C., Leibiger, C., Gentier, R.J., Dumit, V.I., Paduch, K., Eisenberg, T., Habernig, L., Trausinger, G., Magnes, C., Pieber, T., Sinner, F., Dengjel, J., Van Leeuwen, F.W., Kroemer, G., and Madeo, F. (2015). Accumulation of Basic Amino Acids at Mitochondria Dictates the Cytotoxicity of Aberrant Ubiquitin. Cell Reports 9, 1557-1571.

Ciechanover, A. (2005). Proteolysis: from the lysosome to ubiquitin and the proteasome. Nat Rev $\mathrm{Mol}$ Cell Biol 6, 79-87.

Ciechanover, A. (2014). The complexity of recognition of ubiquitinated substrates by the $26 \mathrm{~S}$ proteasome. Biochim Biophys Acta 1843, 86-96.

Ciechanover, A. (2015). Degradation of misfolded proteins in neurodegenerative diseases: therapeutic targets and strategies. Exp Mol Med 47, e147.

De Pril, R., Fischer, D.F., Maat-Schieman, M.L., Hobo, B., De Vos, R.A., Brunt, E.R., Hol, E.M., Roos, R.A., and Van Leeuwen, F.W. (2004). Accumulation of aberrant ubiquitin induces aggregate formation and cell death in polyglutamine diseases. Human molecular genetics 13, 18031813.

De Strooper, B. (2014). Lessons from a failed gamma-secretase Alzheimer trial. Cell 159, 721-726.

De Vrij, F.M., Fischer, D.F., Van Leeuwen, F.W., and Hol, E.M. (2004). Protein quality control in Alzheimer's disease by the ubiquitin proteasome system. Prog Neurobiol 74, 249-270.

Dennissen, F.J., Kholod, N., Hermes, D.J., Kemmerling, N., Steinbusch, H.W., Dantuma, N.P., and Van Leeuwen, F.W. (2011). Mutant ubiquitin (UBB+1) associated with neurodegenerative disorders is hydrolyzed by ubiquitin C-terminal hydrolase L3 (UCH-L3). FEBS Lett 585, 2568-2574. 
Dennissen, F.J., Kholod, N., and Van Leeuwen, F.W. (2012). The ubiquitin proteasome system in neurodegenerative diseases: culprit, accomplice or victim? Progress in neurobiology 96, 190-207.

Drachman, D.A. (2014). The amyloid hypothesis, time to move on: Amyloid is the downstream result, not cause, of Alzheimer's disease. Alzheimers Dement 10, 372-380.

Duyckaerts, C., Delatour, B., and Potier, M.C. (2009). Classification and basic pathology of Alzheimer disease. Acta Neuropathol 118, 5-36.

Fan, J.B., Arimoto, K.L., Motamedchaboki, K., Yan, M., Wolf, D.A., and Zhang, D.E. (2015). Identification and characterization of a novel ISG15-ubiquitin mixed chain and its role in regulating protein homeostasis. Nature Scientific Reports 5, 12704.

Fischer, D.F., De Vos, R.A., Van Dijk, R., De Vrij, F.M., Proper, E.A., Sonnemans, M.A., Verhage, M.C., Sluijs, J.A., Hobo, B., Zouambia, M., Steur, E.N., Kamphorst, W., Hol, E.M., and Van Leeuwen, F.W. (2003). Disease-specific accumulation of mutant ubiquitin as a marker for proteasomal dysfunction in the brain. FASEB journal : official publication of the Federation of American Societies for Experimental Biology 17, 2014-2024.

Fischer, D.F., Van Dijk, R., Van Tijn, P., Hobo, B., Verhage, M.C., Van Der Schors, R.C., Li, K.W., Van Minnen, J., Hol, E.M., and Van Leeuwen, F.W. (2009). Long-term proteasome dysfunction in the mouse brain by expression of aberrant ubiquitin. Neurobiology of Aging 30, 847-863.

Gerez, L., De Haan, A., Hol, E.M., Fischer, D.F., Van Leeuwen, F.W., Van Steeg, H., and Benne, R. (2005). Molecular misreading: the frequency of dinucleotide deletions in neuronal mRNAs for beta-amyloid precursor protein and ubiquitin B. Neurobiol Aging 26, 145-155.

Glenner, G.G., and Wong, C.W. (1984). Alzheimer's disease and Down's syndrome: sharing of a unique cerebrovascular amyloid fibril protein. Biochem Biophys Res Commun 122, 11311135.

Goedert, M., Ghetti, B., and Spillantini, M.G. (2012). Frontotemporal dementia: implications for understanding Alzheimer disease. Cold Spring Harb Perspect Med 2, a006254.

Gregori, L., Fuchs, C., Figueiredo-Pereira, M.E., Van Nostrand, W.E., and Goldgaber, D. (1995). Amyloid beta-protein inhibits ubiquitin-dependent protein degradation in vitro. $\mathrm{J} \mathrm{Biol} \mathrm{Chem}$ 270, 19702-19708.

Haass, C., Kaether, C., Thinakaran, G., and Sisodia, S. (2012). Trafficking and proteolytic processing of APP. Cold Spring Harb Perspect Med 2, a006270.

Hadian, K., Griesbach, R.A., Dornauer, S., Wanger, T.M., Nagel, D., Metlitzky, M., Beisker, W., Schmidt-Supprian, M., and Krappmann, D. (2011). NF-kappaB essential modulator (NEMO) interaction with linear and lys-63 ubiquitin chains contributes to NF-kappaB activation. $J$ Biol Chem 286, 26107-26117.

Harvey, R.J., Skelton-Robinson, M., and Rossor, M.N. (2003). The prevalence and causes of dementia in people under the age of 65 years. J Neurol Neurosurg Psychiatry 74, 12061209.

Heneka, M.T., Ramanathan, M., Jacobs, A.H., Dumitrescu-Ozimek, L., Bilkei-Gorzo, A., Debeir, T., Sastre, M., Galldiks, N., Zimmer, A., Hoehn, M., Heiss, W.D., Klockgether, T., and Staufenbiel, M. (2006). Locus ceruleus degeneration promotes Alzheimer pathogenesis in amyloid precursor protein 23 transgenic mice. The Journal of neuroscience : the official journal of the Society for Neuroscience 26, 1343-1354. 
Hol, E.M., Van Leeuwen, F.W., and Fischer, D.F. (2005). The proteasome in Alzheimer's disease and Parkinson's disease: lessons from ubiquitin B+1. Trends Mol Med 11, 488-495.

Holmes, C., Boche, D., Wilkinson, D., Yadegarfar, G., Hopkins, V., Bayer, A., Jones, R.W., Bullock, R., Love, S., Neal, J.W., Zotova, E., and Nicoll, J.A. (2008). Long-term effects of Abeta42 immunisation in Alzheimer's disease: follow-up of a randomised, placebo-controlled phase I trial. Lancet 372, 216-223.

Holton, P., Ryten, M., and Nalls, M. (2013). Initial assessment of the pathogenic mechanisms of the recently identified Alzheimer risk Loci. Annals of human genetics 77, 85-105.

Hoogendijk, W.J., Pool, C.W., Troost, D., Van Zwieten, E., and Swaab, D.F. (1995). Image analyserassisted morphometry of the locus coeruleus in Alzheimer's disease, Parkinson's disease and amyotrophic lateral sclerosis. Brain 118 ( Pt 1), 131-143.

Hope, A.D., De Silva, R., Fischer, D.F., Hol, E.M., Van Leeuwen, F.W., and Lees, A.J. (2003). Alzheimer's associated variant ubiquitin causes inhibition of the $26 \mathrm{~S}$ proteasome and chaperone expression. Journal of Neurochemistry 86, 394-404.

Hoppe, T. (2005). Multiubiquitylation by E4 enzymes: 'one size' doesn't fit all. Trends Biochem Sci 30, 183-187.

Igap (2015). International Genomics of Alzheimer's Disease Consortium. Convergent genetic and expression data implicate immunity in Alzheimer's disease. Alzheimer's \& Dementia 11, 658-671.

Ihara, Y., Morishima-Kawashima, M., and Nixon, R. (2012). The ubiquitin-proteasome system and the autophagic-lysosomal system in Alzheimer disease. Cold Spring Harb Perspect Med 2.

Iqbal, K., Liu, F., Gong, C.X., Alonso Adel, C., and Grundke-lqbal, I. (2009). Mechanisms of tauinduced neurodegeneration. Acta Neuropathol 118, 53-69.

Irmler, M., Gentier, R.J., Dennissen, F.J., Schulz, H., Bolle, I., Holter, S.M., Kallnik, M., Cheng, J.J., Klingenspor, M., Rozman, J., Ehrhardt, N., Hermes, D.J., Gailus-Durner, V., Fuchs, H., Hrabe De Angelis, M., Meyer, H.E., Hopkins, D.A., Van Leeuwen, F.W., and Beckers, J. (2012). Long-term proteasomal inhibition in transgenic mice by UBB $(+1)$ expression results in dysfunction of central respiration control reminiscent of brainstem neuropathology in Alzheimer patients. Acta neuropathologica 124, 187-197.

Jansen, W.J., Ossenkoppele, R., Knol, D.L., Tijms, B.M., Scheltens, P., Verhey, F.R., Visser, P.J., Amyloid Biomarker Study, G., Aalten, P., Aarsland, D., Alcolea, D., Alexander, M., Almdahl, I.S., Arnold, S.E., Baldeiras, I., Barthel, H., Van Berckel, B.N., Bibeau, K., Blennow, K., Brooks, D.J., Van Buchem, M.A., Camus, V., Cavedo, E., Chen, K., Chetelat, G., Cohen, A.D., Drzezga, A., Engelborghs, S., Fagan, A.M., Fladby, T., Fleisher, A.S., Van Der Flier, W.M., Ford, L., Forster, S., Fortea, J., Foskett, N., Frederiksen, K.S., Freund-Levi, Y., Frisoni, G.B., Froelich, L., Gabryelewicz, T., Gill, K.D., Gkatzima, O., Gomez-Tortosa, E., Gordon, M.F., Grimmer, T., Hampel, H., Hausner, L., Hellwig, S., Herukka, S.K., Hildebrandt, H., Ishihara, L., Ivanoiu, A., Jagust, W.J., Johannsen, P., Kandimalla, R., Kapaki, E., Klimkowicz-Mrowiec, A., Klunk, W.E., Kohler, S., Koglin, N., Kornhuber, J., Kramberger, M.G., Van Laere, K., Landau, S.M., Lee, D.Y., De Leon, M., Lisetti, V., Lleo, A., Madsen, K., Maier, W., Marcusson, J., Mattsson, N., De Mendonca, A., Meulenbroek, O., Meyer, P.T., Mintun, M.A., Mok, V., Molinuevo, J.L., Mollergard, H.M., Morris, J.C., Mroczko, B., Van Der Mussele, S., Na, D.L., Newberg, A., Nordberg, A., Nordlund, A., Novak, G.P., Paraskevas, G.P., Parnetti, L., Perera, G., Peters, O., Popp, J., Prabhakar, S., Rabinovici, G.D., Ramakers, I.H., Rami, L., Resende De Oliveira, C., Rinne, J.O., Rodrigue, 
K.M., et al. (2015). Prevalence of cerebral amyloid pathology in persons without dementia: a meta-analysis. JAMA 313, 1924-1938.

Jellinger, K.A. (2009). Recent advances in our understanding of neurodegeneration. J Neural Transm 116, 1111-1162.

Jonsson, T., Atwal, J.K., Steinberg, S., Snaedal, J., Jonsson, P.V., Bjornsson, S., Stefansson, H., Sulem, P., Gudbjartsson, D., Maloney, J., Hoyte, K., Gustafson, A., Liu, Y., Lu, Y., Bhangale, T., Graham, R.R., Huttenlocher, J., Bjornsdottir, G., Andreassen, O.A., Jonsson, E.G., Palotie, A., Behrens, T.W., Magnusson, O.T., Kong, A., Thorsteinsdottir, U., Watts, R.J., and Stefansson, K. (2012). A mutation in APP protects against Alzheimer's disease and age-related cognitive decline. Nature 488, 96-99.

Jonsson, T., Stefansson, H., Steinberg, S., Jonsdottir, I., Jonsson, P.V., Snaedal, J., Bjornsson, S., Huttenlocher, J., Levey, A.I., Lah, J.J., Rujescu, D., Hampel, H., Giegling, I., Andreassen, O.A., Engedal, K., Ulstein, I., Djurovic, S., Ibrahim-Verbaas, C., Hofman, A., Ikram, M.A., Van Duijn, C.M., Thorsteinsdottir, U., Kong, A., and Stefansson, K. (2013). Variant of TREM2 associated with the risk of Alzheimer's disease. The New England journal of medicine 368, 107-116.

Jucker, M., and Christen, Y. (2013). Proteopathic seeds and neurodegenerative diseases. Springer Heidelberg.

Kadavath, H., Hofele, R.V., Biernat, J., Kumar, S., Tepper, K., Urlaub, H., Mandelkow, E., and Zweckstetter, M. (2015). Tau stabilizes microtubules by binding at the interface between tubulin heterodimers. Proc Natl Acad Sci U S A.

Keck, S., Nitsch, R., Grune, T., and Ullrich, O. (2003). Proteasome inhibition by paired helical filament-tau in brains of patients with Alzheimer's disease. J Neurochem 85, 115-122.

Keller, J.N., Hanni, K.B., and Markesbery, W.R. (2000). Impaired proteasome function in Alzheimer's disease. J Neurochem 75, 436-439.

Kim, H.T., Kim, K.P., Uchiki, T., Gygi, S.P., and Goldberg, A.L. (2009). S5a promotes protein degradation by blocking synthesis of nondegradable forked ubiquitin chains. EMBO J 28, 1867-1877.

Ko, S., Kang, G.B., Song, S.M., Lee, J.G., Shin, D.Y., Yun, J.H., Sheng, Y., Cheong, C., Jeon, Y.H., Jung, Y.K., Arrowsmith, C.H., Avvakumov, G.V., Dhe-Paganon, S., Yoo, Y.J., Eom, S.H., and Lee, W. (2010). Structural basis of E2-25K/UBB+1 interaction leading to proteasome inhibition and neurotoxicity. J Biol Chem 285, 36070-36080.

Koegl, M., Hoppe, T., Schlenker, S., Ulrich, H.D., Mayer, T.U., and Jentsch, S. (1999). A novel ubiquitination factor, E4, is involved in multiubiquitin chain assembly. Cell 96, 635-644.

Komander, D., Clague, M.J., and Urbe, S. (2009). Breaking the chains: structure and function of the deubiquitinases. Nat Rev Mol Cell Biol 10, 550-563.

Kostova, Z., and Wolf, D.H. (2003). For whom the bell tolls: protein quality control of the endoplasmic reticulum and the ubiquitin-proteasome connection. EMBO J 22, 2309-2317.

Krutauz, D., Reis, N., Nakasone, M.A., Siman, P., Zhang, D., Kirkpatrick, D.S., Gygi, S.P., Brik, A., Fushman, D., and Glickman, M.H. (2014). Extended ubiquitin species are protein-based DUB inhibitors. Nat Chem Biol 10, 664-670.

Lam, Y.A., Pickart, C.M., Alban, A., Landon, M., Jamieson, C., Ramage, R., Mayer, R.J., and Layfield, R. (2000). Inhibition of the ubiquitin-proteasome system in Alzheimer's disease. 
Proceedings of the National Academy of Sciences of the United States of America 97, 99029906.

Lannfelt, L., Moller, C., Basun, H., Osswald, G., Sehlin, D., Satlin, A., Logovinsky, V., and Gellerfors, P. (2014). Perspectives on future Alzheimer therapies: amyloid-beta protofibrils - a new target for immunotherapy with BAN2401 in Alzheimer's disease. Alzheimers Res Ther 6, 16.

Layfield, R., Lowe, J., and Bedford, L. (2005). The ubiquitin-proteasome system and neurodegenerative disorders. Essays Biochem 41, 157-171.

Lee, J.H., Ryan, J., Andreescu, C., Aizenstein, H., and Lim, H.K. (2015). Brainstem morphological changes in Alzheimer's disease. Neuroreport 26, 411-415.

Li, M., Wang, I.X., and Cheung, V.G. (2012). Response to comments on "Widespread RNA and DNA sequence differences in the human transcriptome". 335.

Li, M., Wang, I.X., Li, Y., Bruzel, A., Richards, A.L., Toung, J.M., and Cheung, V.G. (2011). Widespread RNA and DNA sequence differences in the human transcriptome. Science 333, 53-58.

Lindsten, K., De Vrij, F.M., Verhoef, L.G., Fischer, D.F., Van Leeuwen, F.W., Hol, E.M., Masucci, M.G., and Dantuma, N.P. (2002). Mutant ubiquitin found in neurodegenerative disorders is a ubiquitin fusion degradation substrate that blocks proteasomal degradation. The Journal of cell biology 157, 417-427.

Lomas, D.A., and Carrell, R.W. (2002). Serpinopathies and the conformational dementias. Nat Rev Genet 3, 759-768.

Lopez Salon, M., Morelli, L., Castano, E.M., Soto, E.F., and Pasquini, J.M. (2000). Defective ubiquitination of cerebral proteins in Alzheimer's disease. $J$ Neurosci Res 62, 302-310.

Lopez Salon, M., Pasquini, L., Besio Moreno, M., Pasquini, J.M., and Soto, E. (2003). Relationship between beta-amyloid degradation and the $26 \mathrm{~S}$ proteasome in neural cells. Exp Neurol 180, 131-143.

Manavalan, A., Mishra, M., Feng, L., Sze, S.K., Akatsu, H., and Heese, K. (2013). Brain site-specific proteome changes in aging-related dementia. Experimental \& Molecular Medicine 45, e39.

Mandelkow, E.M., and Mandelkow, E. (2012). Biochemistry and cell biology of tau protein in neurofibrillary degeneration. Cold Spring Harb Perspect Med 2, a006247.

Mann, D.M., Lincoln, J., Yates, P.O., Stamp, J.E., and Toper, S. (1980). Changes in the monoamine containing neurones of the human CNS in senile dementia. Br J Psychiatry 136, 533-541.

Maquat, L.E. (2015). The amazing web of post-transcriptional gene control: the sum of small changes can make for significant consequences. RNA 21, 488-489.

Maslow, A.H. (1943). A Theory of Human Motivation. Psychological Review 50, 370-396.

Masters, C.L., and Selkoe, D.J. (2012). Biochemistry of amyloid beta-protein and amyloid deposits in Alzheimer disease. Cold Spring Harb Perspect Med 2, a006262.

Matsumoto, M.L., Wickliffe, K.E., Dong, K.C., Yu, C., Bosanac, I., Bustos, D., Phu, L., Kirkpatrick, D.S., Hymowitz, S.G., Rape, M., Kelley, R.F., and Dixit, V.M. (2010). K11-linked polyubiquitination in cell cycle control revealed by a K11 linkage-specific antibody. Mol Cell 39, 477-484.

Mercy, L., Hodges, J.R., Dawson, K., Barker, R.A., and Brayne, C. (2008). Incidence of early-onset dementias in Cambridgeshire, United Kingdom. Neurology 71, 1496-1499. 
Morishima-Kawashima, M., Hasegawa, M., Takio, K., Suzuki, M., Titani, K., and Ihara, Y. (1993). Ubiquitin is conjugated with amino-terminally processed tau in paired helical filaments. Neuron 10, 1151-1160.

Morris, M., Maeda, S., Vossel, K., and Mucke, L. (2011). The many faces of tau. Neuron 70, 410426.

Mucke, L., and Selkoe, D.J. (2012). Neurotoxicity of amyloid beta-protein: synaptic and network dysfunction. Cold Spring Harb Perspect Med 2, a006338.

Nagy, E., and Maquat, L.E. (1998). A rule for termination-codon position within intron-containing genes: when nonsense affects RNA abundance. Trends Biochem Sci 23, 198-199.

Nixon, R.A. (2013). The role of autophagy in neurodegenerative disease. Nat Med 19, 983-997.

Olsson, A., Hoglund, K., Sjogren, M., Andreasen, N., Minthon, L., Lannfelt, L., Buerger, K., Moller, H.J., Hampel, H., Davidsson, P., and Blennow, K. (2003). Measurement of alpha- and betasecretase cleaved amyloid precursor protein in cerebrospinal fluid from Alzheimer patients. Exp Neurol 183, 74-80.

Park, Y., Yoon, S.K., and Yoon, J.B. (2009). The HECT domain of TRIP12 ubiquitinates substrates of the ubiquitin fusion degradation pathway. J Biol Chem 284, 1540-1549.

Peng, J., Schwartz, D., Elias, J.E., Thoreen, C.C., Cheng, D., Marsischky, G., Roelofs, J., Finley, D., and Gygi, S.P. (2003). A proteomics approach to understanding protein ubiquitination. Nat Biotechnol 21, 921-926.

Pericak-Vance, M.A., Bass, M.P., Yamaoka, L.H., Gaskell, P.C., Scott, W.K., Terwedow, H.A., Menold, M.M., Conneally, P.M., Small, G.W., Vance, J.M., Saunders, A.M., Roses, A.D., and Haines, J.L. (1997). Complete genomic screen in late-onset familial Alzheimer disease. Evidence for a new locus on chromosome 12. JAMA 278, 1237-1241.

Peth, A., Nathan, J.A., and Goldberg, A.L. (2013). The ATP costs and time required to degrade ubiquitinated proteins by the 26 S proteasome. J Biol Chem 288, 29215-29222.

Pickart, C.M. (2001). Mechanisms underlying ubiquitination. Annu Rev Biochem 70, 503-533.

Poulsen, E.G., Steinhauer, C., Lees, M., Lauridsen, A.M., Ellgaard, L., and Hartmann-Petersen, R. (2012). HUWE1 and TRIP12 collaborate in degradation of ubiquitin-fusion proteins and misframed ubiquitin. PLoS One 7, e50548.

Querfurth, H.W., and Laferla, F.M. (2010). Alzheimer's disease. N Engl J Med 362, 329-344.

Regeur, L., Jensen, G.B., Pakkenberg, H., Evans, S.M., and Pakkenberg, B. (1994). No global neocortical nerve cell loss in brains from patients with senile dementia of Alzheimer's type. Neurobiol Aging 15, 347-352.

Reiman, E.M., Quiroz, Y.T., Fleisher, A.S., Chen, K., Velez-Pardo, C., Jimenez-Del-Rio, M., Fagan, A.M., Shah, A.R., Alvarez, S., Arbelaez, A., Giraldo, M., Acosta-Baena, N., Sperling, R.A., Dickerson, B., Stern, C.E., Tirado, V., Munoz, C., Reiman, R.A., Huentelman, M.J., Alexander, G.E., Langbaum, J.B., Kosik, K.S., Tariot, P.N., and Lopera, F. (2012). Brain imaging and fluid biomarker analysis in young adults at genetic risk for autosomal dominant Alzheimer's disease in the presenilin 1 E280A kindred: a case-control study. Lancet Neurol 11, 1048-1056.

Rovelet-Lecrux, A., Hannequin, D., Raux, G., Le Meur, N., Laquerriere, A., Vital, A., Dumanchin, C., Feuillette, S., Brice, A., Vercelletto, M., Dubas, F., Frebourg, T., and Campion, D. (2006). 
APP locus duplication causes autosomal dominant early-onset Alzheimer disease with cerebral amyloid angiopathy. Nat Genet 38, 24-26.

Saido, T., and Leissring, M.A. (2012). Proteolytic degradation of amyloid beta-protein. Cold Spring Harb Perspect Med 2, 1-18.

Sankaranarayanan, S., Barten, D.M., Vana, L., Devidze, N., Yang, L., Cadelina, G., Hoque, N., Decarr, L., Keenan, S., Lin, A., Cao, Y., Snyder, B., Zhang, B., Nitla, M., Hirschfeld, G., Barrezueta, N., Polson, C., Wes, P., Rangan, V.S., Cacace, A., Albright, C.F., Meredith, J., Jr., Trojanowski, J.Q., Lee, V.M., Brunden, K.R., and Ahlijanian, M. (2015). Passive immunization with phospho-tau antibodies reduces tau pathology and functional deficits in two distinct mouse tauopathy models. PLoS One 10, e0125614.

Schipanski, A., Oberhauser, F., Neumann, M., Lange, S., Szalay, B., Krasemann, S., Van Leeuwen, F.W., Galliciotti, G., and Glatzel, M. (2014). The lectin OS-9 delivers mutant neuroserpin to endoplasmic reticulum associated degradation in familial encephalopathy with neuroserpin inclusion bodies. Neurobiol Aging 35, 2394-2403.

Selkoe, D.J. (2001). Alzheimer's disease: genes, proteins, and therapy. Physiol Rev 81, 741-766.

Selkoe, D.J., Mandelkow, E., and Holtzman, D.M. (2012). The biology of Alzheimer's disease. Cold Spring Harb Perspect Med 1. ISSN 2157-1422.

Serrano-Pozo, A., Frosch, M.P., Masliah, E., and Hyman, B.T. (2011). Neuropathological alterations in Alzheimer disease. Cold Spring Harb Perspect Med 1, a006189.

Simic, G., Stanic, G., Mladinov, M., Jovanov-Milosevic, N., Kostovic, I., and Hof, P.R. (2009). Does Alzheimer's disease begin in the brainstem? Neuropathol Appl Neurobiol 35, 532-554.

Sleegers, K., Brouwers, N., Gijselinck, I., Theuns, J., Goossens, D., Wauters, J., Del-Favero, J., Cruts, M., Van Duijn, C.M., and Van Broeckhoven, C. (2006). APP duplication is sufficient to cause early onset Alzheimer's dementia with cerebral amyloid angiopathy. Brain 129, 2977-2983.

Tan, Z., Sun, X., Hou, F.S., Oh, H.W., Hilgenberg, L.G., Hol, E.M., Van Leeuwen, F.W., Smith, M.A., O'dowd, D.K., and Schreiber, S.S. (2007). Mutant ubiquitin found in Alzheimer's disease causes neuritic beading of mitochondria in association with neuronal degeneration. Cell Death Differ 14, 1721-1732.

Thies, W. (2012). 2012 Alzheimer's disease facts and figures. Alzheimer's \& dementia : the journal of the Alzheimer's Association 8, 131-168.

Thrower, J.S., Hoffman, L., Rechsteiner, M., and Pickart, C.M. (2000). Recognition of the polyubiquitin proteolytic signal. EMBO J 19, 94-102.

Todi, S.V., and Paulson, H.L. (2011). Balancing act: deubiquitinating enzymes in the nervous system. Trends Neurosci 34, 370-382.

Tsakiri, E.N., and Trougakos, I.P. (2015). The amazing ubiquitin-proteasome system: structural components and implication in aging. Int Rev Cell Mol Biol 314, 171-237.

Tseng, B.P., Green, K.N., Chan, J.L., Blurton-Jones, M., and Laferla, F.M. (2008). Abeta inhibits the proteasome and enhances amyloid and tau accumulation. Neurobiol Aging 29, 1607-1618.

Tucker, S.M., Borchelt, D.R., and Troncoso, J.C. (2008). Limited clearance of pre-existing amyloid plaques after intracerebral injection of Abeta antibodies in two mouse models of Alzheimer disease. J Neuropathol Exp Neurol 67, 30-40. 
Van Leeuwen, F.W., De Kleijn, D.P., Van Den Hurk, H.H., Neubauer, A., Sonnemans, M.A., Sluijs, J.A., Koycu, S., Ramdjielal, R.D., Salehi, A., Martens, G.J., Grosveld, F.G., Peter, J., Burbach, H., and Hol, E.M. (1998). Frameshift mutants of beta amyloid precursor protein and ubiquitin-B in Alzheimer's and Down patients. Science 279, 242-247.

Van Leeuwen, F.W., Fischer, D.F., Kamel, D., Sluijs, J.A., Sonnemans, M.A., Benne, R., Swaab, D.F., Salehi, A., and Hol, E.M. (2000). Molecular misreading: a new type of transcript mutation expressed during aging. Neurobiol Aging 21, 879-891.

Van Leeuwen, F.W., Van Tijn, P., Sonnemans, M.A., Hobo, B., Mann, D.M., Van Broeckhoven, C., Kumar-Singh, S., Cras, P., Leuba, G., Savioz, A., Maat-Schieman, M.L., Yamaguchi, H., Kros, J.M., Kamphorst, W., Hol, E.M., De Vos, R.A., and Fischer, D.F. (2006). Frameshift proteins in autosomal dominant forms of Alzheimer disease and other tauopathies. Neurology 66, S86-92.

Van Tijn, P., De Vrij, F.M., Schuurman, K.G., Dantuma, N.P., Fischer, D.F., Van Leeuwen, F.W., and Hol, E.M. (2007). Dose-dependent inhibition of proteasome activity by a mutant ubiquitin associated with neurodegenerative disease. Journal of Cell Science 120, 1615-1623.

Van Tijn, P., Dennissen, F.J., Gentier, R.J., Hobo, B., Hermes, D., Steinbusch, H.W., Van Leeuwen, F.W., and Fischer, D.F. (2012). Mutant ubiquitin decreases amyloid beta plaque formation in a transgenic mouse model of Alzheimer's disease. Neurochemistry international 61, 739748.

Verhoef, L.G., Heinen, C., Selivanova, A., Halff, E.F., Salomons, F.A., and Dantuma, N.P. (2009). Minimal length requirement for proteasomal degradation of ubiquitin-dependent substrates. FASEB J 23, 123-133.

Vermulst, M., Denney, A.S., Lang, M.J., Hung, C.W., Moore, S., Mosely, A.M., Thompson, W.J., Madden, V., Gauer, J., Wolfe, K.J., Summers, D.W., Schleit, J., Sutphin, G.L., Haroon, S., Holczbauer, A., Caine, J., Jorgenson, J., Cyr, D., Kaeberlein, M., Strathern, J.N., Duncan, M.C., and Erie, D.A. (2015). Transcription errors induce proteotoxic stress and shorten cellular lifespan. Nat Commun 6, 8065.

Wang, I.X., Core, L.J., Kwak, H., Brady, L., Bruzel, A., Mcdaniel, L., Richards, A.L., Wu, M., Grunseich, C., Lis, J.T., and Cheung, V.G. (2014). RNA-DNA differences are generated in human cells within seconds after RNA exits polymerase II. Cell Rep 6, 906-915.

Weksler, M.E., Szabo, P., Relkin, N.R., Reidenberg, M.M., Weksler, B.B., and Coppus, A.M. (2013). Alzheimer's disease and Down's syndrome: treating two paths to dementia. Autoimmun Rev 12, 670-673.

West, M.J., Coleman, P.D., Flood, D.G., and Troncoso, J.C. (1994). Differences in the pattern of hippocampal neuronal loss in normal ageing and Alzheimer's disease. Lancet 344, 769-772.

Wolf, D.H., and Hilt, W. (2004). The proteasome: a proteolytic nanomachine of cell regulation and waste disposal. Biochim Biophys Acta 1695, 19-31.

Zouambia, M., Fischer, D.F., Hobo, B., De Vos, R.A., Hol, E.M., Varndell, I.M., Sheppard, P.W., and Van Leeuwen, F.W. (2008). Proteasome subunit proteins and neuropathology in tauopathies and synucleinopathies: Consequences for proteomic analyses. Proteomics 8, 1221-1236. 



\section{CHAPTER 2}

Localization of mutant ubiquitin in the brain of a transgenic mouse line with proteasomal inhibition and its validation at specific sites in Alzheimer's disease

R.J. Gentier, B.M. Verheijen, M. Zamboni, M.M.A. Stroeken, D.J.H.P. Hermes, B. Küsters, H.W.M. Steinbusch, D.A. Hopkins and F.W. Van Leeuwen, Frontiers in Neuroanatomy.2015;9:1-21

Supplementary material available at:

http://journal.frontiersin.org/article/10.3389/fnana.2015.00026/abstract 


\section{Abstract}

Loss of protein quality control by the ubiquitin-proteasome system (UPS) during aging is one of the processes putatively contributing to cellular stress and Alzheimer's disease (AD) pathogenesis. Recently, pooled Genome Wide Association Studies (GWAS), pathway analysis and proteomics identified protein ubiquitination as one of the key modulators of $A D$. Mutations in ubiquitin $B$ mRNA that result in $U_{B B}{ }^{+1}$ dose-dependently cause an impaired UPS, subsequent accumulation of $\mathrm{UBB}^{+1}$ and most probably depositions of other aberrant proteins present in plaques and neurofibrillary tangles.

We used specific immunohistochemical probes for a comprehensive topographic mapping of the $\mathrm{UBB}^{+1}$ distribution in the brains of transgenic mouse line 3413 overexpressing $\mathrm{UBB}^{+1}$. We also mapped the expression of $\mathrm{UBB}^{+1}$ in brain areas of $A D$ patients selected based upon the distribution of $\mathrm{UBB}^{+1}$ in line 3413. Therefore we focused on the olfactory bulb, basal ganglia, nucleus basalis of Meynert, inferior colliculus and raphe nuclei. $\mathrm{UBB}^{+1}$ distribution was compared with established probes for pre-tangles and tangles and $A \beta$ plaques.

$\mathrm{UBB}^{+1}$ distribution found in line 3413 is partly mirrored in the AD brain. Specifically, nuclei with substantial accumulations of tangle-bearing neurons, such as the nucleus basalis of Meynert and raphe nuclei also present high densities of $\mathrm{UBB}^{+1}$ positive tangles.

Line 3413 is useful for studying the contribution of proteasomal dysfunction in AD. The findings are consistent with evidence that areas outside the forebrain are also affected in AD. Line 3413 may also be predictive for other conformational diseases, including related tauopathies and polyglutamine diseases, in which $\mathrm{UBB}^{+1}$ accumulates in their cellular hallmarks. 


\section{Introduction}

Alzheimer's disease (AD) is a multifactorial disease and the most prevalent form of dementia. Currently, it is estimated to affect at least 20 million people worldwide and the prevalence is expected to triple within the next 40 years (Barnes and Yaffe, 2011). AD neuropathology is characterized by two cellular hallmarks: the accumulation of extracellular plaques mainly composed of amyloid $\beta(A \beta)$ and of intracellular hyperphosphorylated tau in neurofibrillary tangles (NFTs) (Selkoe, 2001). In autosomal dominant AD cases (accounting for <3\%) (van Leeuwen et al., 2000), mutations in three different genes have been identified: the amyloid precursor protein (APP) and the presenilin 1 (PS1) and 2 (PS2) genes. Pathogenic mutations in these genes contribute to aberrations of the $\gamma$-secretase complex, which leads to an increased production of toxic $A \beta_{42}$ (Jankowsky et al., 2004). In the tau gene of AD patients no mutations have been reported so far. The major risk factor for developing sporadic $A D$ is aging whereas the $\varepsilon 4$ polymorphism of the apolipoprotein $E$ gene (APOE) is the most prominent genetic risk factor (Corder et al., 1993). Other mediumrisk factors such as a missense mutation in the gene encoding the triggering receptor expressed on myeloid cells 2 (TREM2) (Jonsson et al., 2013) and nine low risk factors from Genome Wide Association Studies (GWAS) were reported to contribute to AD (Holton et al., 2013). Recently, pooled GWAS studies and pathway analysis also identified protein ubiquitination as one of the key modulators of AD (IGAP, 2015). In this study they implicate that the regulation of endocytosis and protein ubiquitination, in addition to cholesterol metabolism are potential therapeutic targets in AD. In addition, a brain site specific strategy was used to compare the proteomes of prefrontal cortex, hippocampus and cerebellum in brains of AD patients (Manavalan et al., 2013). An Ingenuity Pathway Analysis demonstrated 31 proteins were significantly altered and that these proteins had a strong interaction with the ubiquitin $C$ (UBC) signaling pointing to a dysfunctional ubiquitin proteasome system (UPS) as a causative factor in AD (Manavalan et al., 2013).

Neuronal development and synaptic plasticity are part of processes that involve metabolism of $5-8 \%$ of brain proteins each day (Dennissen et al., 2012). This turnover requires an efficient protein quality control (PQC) for which the UPS and autophagy are mainly responsible. We discovered that the transcription of the ubiquitin $B$ (UBB) gene can 
result in accumulation of mutant ubiquitin $\mathrm{B}^{+1}\left(\mathrm{UBB}^{+1}\right)$ in the cellular hallmarks (plaques and tangles) of sporadic and autosomal dominant $A D$ cases, suggesting a pathological function (van Leeuwen et al., 1998;van Leeuwen et al., 2006). Similarly, other tauopathies, as well as several polyglutamine diseases (e.g., Huntington's diseases (HD)) are characterized by the accumulation of $\mathrm{UBB}^{+1}$ in the respective hallmarks. By contrast, $\mathrm{UBB}^{+1}$ appears not to be involved in synucleinopathies (Fischer et al., 2003;de Pril et al., 2004). UBB ${ }^{+1}$ compromises PQC by inhibiting the UPS dose-dependently (Dennissen et al., 2012) via inhibition of deubiquitinating enzymes (DUB) (Krutauz et al., 2014).

In vivo studies performed in a transgenic (tg) line (\#3413) overexpressing human $\mathrm{UBB}^{+1}$, specifically in neurons postnatally, showed increased levels of ubiquitinated proteins in the forebrain (e.g., cerebral cortex, hippocampus, dentate gyrus, amygdala and striatum). These tg mice show deficits in contextual memory, a decrease in proteasome activity and proteomic changes reminiscent of AD (Fischer et al., 2009). In addition, a comprehensive phenotypic screen of line 3413 revealed a respiratory phenotype (Irmler et al., 2012). Changes in spontaneous breathing patterns and an altered hypoxic response, suggested a central dysfunction of respiratory regulation. In keeping with this, expression of $\mathrm{UBB}^{+1}$ was found in the nucleus of the tractus solitarius (Sol) and the parabrachial nuclei, brainstem nuclei involved in respiratory control. These data suggest that respiratory centres in the brainstem are sensitive to long-term UPS inhibition via the expression of the $\mathrm{UBB}^{+1}$ protein. Most interestingly, $\mathrm{UBB}^{+1}$ immunoreactivity in $\mathrm{AD}$ patients was seen in similar areas as in the tg mice, suggesting a possible functional link between $\mathrm{UBB}^{+1}$ expression in brainstem areas and the respiratory and swallowing dysfunctions that are often seen in $A D$ patients (Irmler et al., 2012). Moreover, in early stages of AD, it has been noted that increased cardiorespiratory fitness in early-stage $A D$ is associated with reduced brain atrophy as compared with non-demented individuals (Burns et al., 2008). It was also shown that declining CR fitness over 2 years was associated with brain atrophy, especially in the parahippocampus in AD (Vidoni et al., 2012). Dysphagia is also an issue in Parkinson's disease (PD) caused by synucleinopathology in the glossopharyngeal nucleus (Braak et al., 2003;Cereda et al., 2014), starts initially in the dorsal motor nucleus of the vagus nerve (Braak et al., 2003) but it is $\mathrm{UBB}^{+1}$ negative. 
Previous studies have concentrated mainly on parts of the forebrain and the brainstem; however, information about $\mathrm{UBB}^{+1}$ accumulation in other brain areas of these tg mice and the possible functional consequences of $\mathrm{UBB}^{+1}$ expression has been lacking. The pattern of $\mathrm{UBB}^{+1}$ expression and expression levels in the tg mice are largely dictated by copy numbers and CamKIla promotor elements used to drive expression, therefore the results of the tg mice must not be overestimated (Fischer et al., 2009). The aim of the present study is to provide a comprehensive topographic mapping of $\mathrm{UBB}^{+1}$ in the brains of the tg mouse line 3413. Due to the extensive $\mathrm{UBB}^{+1}$ expression in certain mouse brain areas, a next step was to compare this distribution with the distribution of $\mathrm{UBB}^{+1}$ and established markers of $A D$ in the human brain. This approach identified two immunoreactive brainstem areas that show a similar immunoreactivity in $A D$ and could be linked to respiratory dysfunction (Irmler et al., 2012). As we detected additional high intensities of $\mathrm{UBB}^{+1}$ immunoreactivity, especially in five other brain areas in line 3413 , we focus here on these brain areas known to be affected in $A D$, namely the olfactory bulb (OB), basal ganglia, nucleus basalis of Meynert (NBM), raphe nuclei and inferior colliculus (IC) and discuss their potential relevance for $A D$ research.

\section{Materials and methods}

\subsection{Animals}

In the present study, $\mathrm{UBB}^{+1}$ tg male mice (line 3413,008833C57BI/6. Tg(CaMKIla-UBB) 3413, Jackson \#008833) ( $n=14$; eight 3-month-old mice, two 7-month-old mice and four 15-month-old mice) expressing human $\mathrm{UBB}^{+1}$ in the postnatal brain on a pure C57BL/6 background were used (Fischer et al., 2009). $\mathrm{UBB}^{+1} \mathrm{CDNA}$ is encoded by the first ubiquitin upstream open reading frame and a murine calmodulin kinase II alpha (CaMKIla) promoter was used to regulate the $\mathrm{UBB}^{+1}$ expression in the tg mice (van Leeuwen et al., 1998). Nontransgenic littermates were used as controls $(n=9)$. To ensure comparability among individuals, mice were kept under standard animal housing conditions: a 12/12 h light- dark cycle with food and acidified water ad libitum in specific pathogen free conditions. 
All animals used in the present study were males and were sacrificed at either 3, 7 and 15 months of age. All animal experiments were performed conforming to national animal welfare law and under guidance of the animal welfare committees of the Royal Netherlands Academy of Arts and Sciences and of Maastricht University (Dier Experimenten Commissie (DEC/ Animal Experiments Commission) protocol nr. 2008-069).

\subsection{Immunohistochemistry}

Mice were deeply anesthetized with sodium pentobarbital and were perfused transcardially with Tyrode solution $\left(2.68 \mathrm{mM} \mathrm{KCl}, 0.245 \mathrm{mM} \mathrm{MgCl} 2.6 \mathrm{H}_{2} \mathrm{O}, 136 \mathrm{mM} \mathrm{NaCl}, 0.289 \mathrm{mM}\right.$ $\mathrm{NaH}_{2} \mathrm{PO}_{4} . \mathrm{H}_{2} \mathrm{O}, 5 \mathrm{mM}$ glucose, $12 \mathrm{mM} \mathrm{NaHCO}_{3} ; \mathrm{pH}^{7.4}$ ) aerated with carbogen followed by $0,1 \mathrm{M}$ phosphate buffer containing $4 \%$ paraformaldehyde $(\mathrm{pH} 7.4)$. Brains were removed and placed in fixative overnight on a rocking table at $4^{\circ} \mathrm{C}$. Subsequently, they were stored in a $1 \%$ sodium azide $\left(\mathrm{NaN}_{3}\right)$ phosphate-buffered saline (PBS) solution in a cold room at $4^{\circ} \mathrm{C}$ until further processing. Brains were embedded in gelatin and sectioned on a Vibratome (Leica VT 1200S, Wetzlar, Germany) into $50 \mu \mathrm{m}$ coronal or sagittal free-floating sections. Tissue sections were stained overnight at $4^{\circ} \mathrm{C}$ (first $1 \mathrm{~h}$ at room temperature (RT)) with a primary polyclonal rabbit anti-UBB ${ }^{+1}$ antibody (Ubi3 16/09/97, final dilution 1:1000), recognizing the C-terminal extension of the $\mathrm{UBB}^{+1}$ protein (Fischer et al., 2003). The sections were incubated with a biotinylated donkey anti-rabbit antibody (1:400) (Jackson Laboratories) followed by avidin-biotin-peroxidase (ABC, 1:400,Vector), both $1 \mathrm{~h}$ at RT. The staining was visualized with 3,3'-diaminobenzidine tetrahydrochloride (DAB) solution intensified by $0.2 \%$ nickel ammonium sulphate $(\mathrm{pH} 7.6)$. The sections were mounted on gelatin-coated glass slides, air dried, dehydrated and coverslipped using Pertex (Histolab). As controls for Ubi3 specificity, Ubi3 antiserum adsorbed with ${ }^{6} \mathrm{His}$ tag $\mathrm{UBB}^{+1}$ as well as the pre-immune control serum were used.

Human postmortem tissue (OB, basal ganglia, NBM, IC and raphe nuclei) from AD patients and non-demented controls was obtained from the Radboud University Medical Center (Department of Pathology, Nijmegen, The Netherlands, Table 1). Anonymised human material from AD-patients and non-demented control patients was used according Dutch law and local guidelines. The number of available human tissue material of the patients is 
different for each of the focused brain areas. The tissues were fixed in $4 \%$ buffered paraformaldehyde for at least 3 weeks. Part of the human OB tissue specimens were cryoprotected in $10 \%$ sucrose solution $\left(0.1 \mathrm{M}\right.$ phosphate buffer, $\mathrm{pH} 7.6$, at $\left.4^{\circ} \mathrm{C}\right)$ followed by at least $72 \mathrm{~h}$ incubation in $20 \%$ sucrose solution. Subsequently, this tissue was frozen and stored at $-80^{\circ} \mathrm{C}$. Coronal sections of $8 \mu \mathrm{m}$ thickness were mounted on gelatin-coated glass slides and stored at $-80^{\circ} \mathrm{C}$ until further processing. The other $\mathrm{OB}$ tissue specimens with olfactory cortex added were dehydrated, embedded in paraffin and cut in the sagittal plane, producing serial sections of $8 \mu \mathrm{m}$ thickness each. Tissue of the basal ganglia, NBM, IC and raphe nuclei were fixed in $4 \%$ buffered paraformaldehyde for 1 month after which the unembedded tissue was cut on a Vibratome in $50 \mu \mathrm{m}$ thick sections and stored at $4^{\circ} \mathrm{C}$ in PBS with $1 \% \mathrm{NaN}_{3}$ until further processing. Basal ganglia were cut in the coronal plane, whereas brainstem (IC and raphe nuclei) was cut perpendicular to the long axis of the spinal cord, to take into account the flexures of the human neuraxis. 


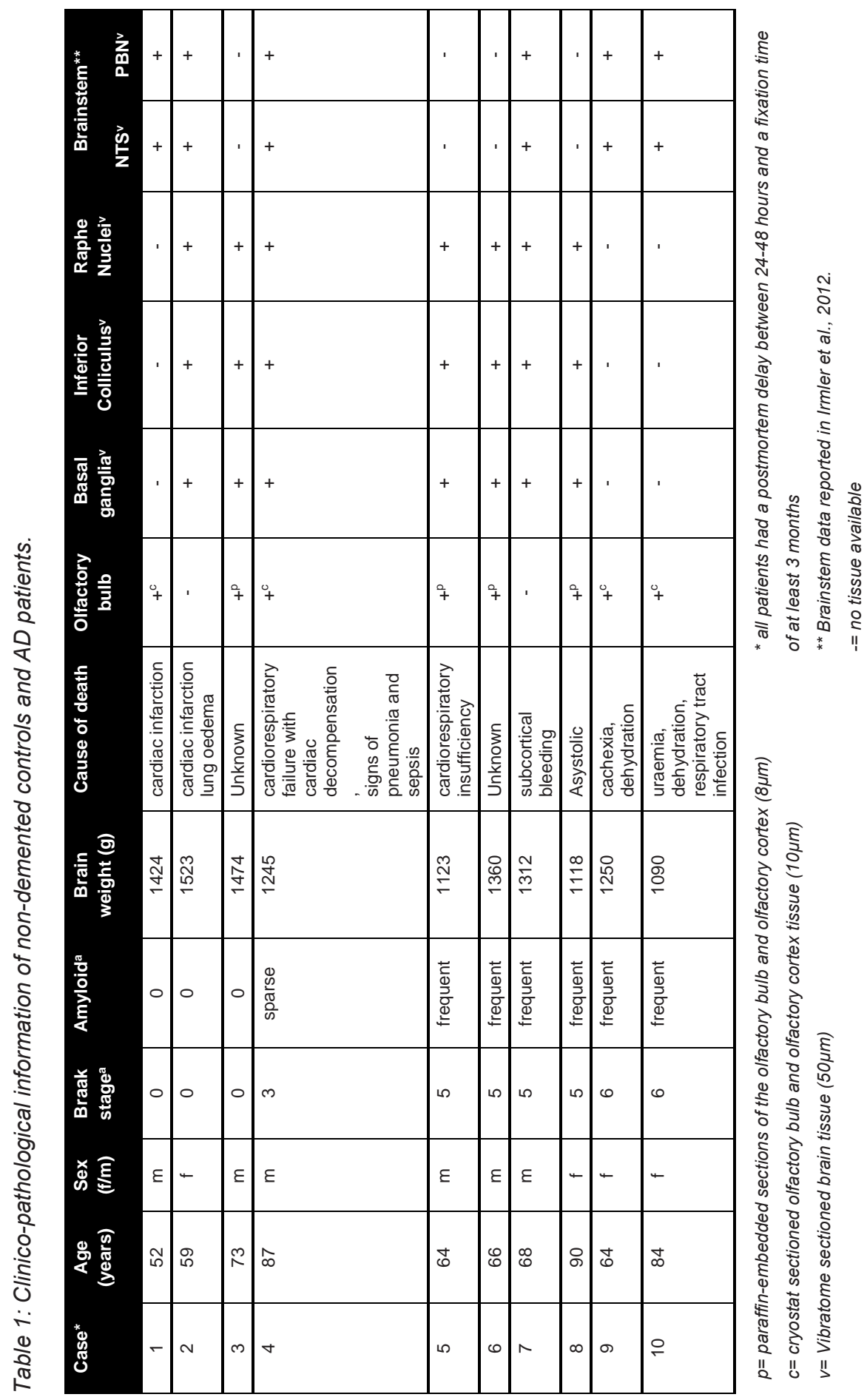


To perform immunohistochemistry, the frozen sections and the paraffin-embedded sections (after deparaffination) were incubated for $30 \mathrm{~min}$ in 100\% formic acid followed by rinsing in distilled water (30 min) and in Tris-buffered saline (TBS) (30 min) and subsequently incubated overnight at $4^{\circ} \mathrm{C}$ (first $1 \mathrm{~h}$ at RT) with antibodies against misfolded tau (monoclonal MC1, 1:100, Dr. P. Davies, New York) (Petry et al., 2014), phosphorylated tau serine 202 (monoclonal CP13, 1:100 Dr. P. Davies, New York) (Petry et al., 2014), amyloid $\beta$ (monoclonal 6F3D,1:100, Dako) and $\mathrm{UBB}^{+1}$ (polyclonal Ubi2A, 180398, 1:400 or polyclonal Ubi2 $\left.{ }^{+1} 140994,1 ; 400\right)$. All dilutions were in Sumi buffer $(0.05 \mathrm{M}$ Tris with $0.9 \%$ $\mathrm{NaCl}, 0.25 \mathrm{M}$ gelatin and 0.5\% Triton X-100, pH 7.4) (Fischer et al., 2003;van Leeuwen et al., 2006). Next, sections were incubated with secondary donkey anti-mouse or donkey anti-rabbit antibodies, both biotinylated (1:400, Jackson Laboratories) and ABC (1:400, Vector), both $1 \mathrm{~h}$ at RT and finally stained with $\mathrm{DAB}$, dehydrated and coverslipped as described above.

Free-floating Vibratome sections were pretreated with $100 \%$ formic acid for the $6 \mathrm{~F} 3 \mathrm{D}$ antibody for $30 \mathrm{~min}$ and then rinsed in distilled water (30min) and TBS (10min). Subsequently, all sections were treated with graded series of methanol $(20 \%, 40 \%, 60 \%$, $80 \%$ (10 min) and 100\% (30 min), and back to $20 \%$ methanol with $0.3 \% \mathrm{H}_{2} \mathrm{O}_{2}$. Rinsing was performed for $30 \mathrm{~min}$ in TBS followed by $30 \mathrm{~min}$ in Sumi buffer. Sections were then incubated with a primary antibody ( $1 \mathrm{~h}$ at RT followed by $36 \mathrm{~h}$ incubation at $4^{\circ} \mathrm{C}$ in a humid chamber), biotinylated secondary antibodies ( $2 \mathrm{~h}$ at RT), ABC (2h at RT) and DAB as described above. All sections were mounted on gelatin-coated glass slides, air dried, dehydrated and coverslipped with DPX (Klinipath). As controls for Ubi2 ${ }^{+1}$ and Ubi2A specificity, Ubi2 ${ }^{+1}$ and Ubi2A antiserum adsorbed with ${ }^{6} \mathrm{His}$ tag $\mathrm{UBB}^{+1}$ as well as the preimmune control serum were used (van Leeuwen et al., 1998;Fischer et al., 2003).

\subsection{Microscopy}

Brain sections of $3413 \mathrm{tg}$ and control mice were analyzed semi-quantitatively using an atlas (Franklin and Paxinos, 2007) and light microscopy, by focusing on intensity (I) of individual neuron staining and density (D) of relative numbers of stained neurons showing $\mathrm{UBB}^{+1}$ immunoreactivity (ir) in a specific brain nucleus. Intensity is defined as the stain quality in 
a specific brain region while density is defined as the number of $\mathrm{UBB}^{+1}$ immunoreactive cells in this region. Scoring was assessed independently by two observers: - no UBB ${ }^{+1}$ ir, + a low I or D of $\mathrm{UBB}^{+1}$-ir cells, ++ a moderate I or D of UBB ${ }^{+1}$-ir cells, +++ a high I or D of $\mathrm{UBB}^{+1}$-ir cells and ++++ a very high I or $\mathrm{D}$ of $\mathrm{UBB}^{+1}$-ir cells. The mean I and $\mathrm{D}$ per brain nuclei were determined semi-quantitatively for final results. Based on these scores, five areas with a high expression of $\mathrm{UBB}^{+1}$ were selected for the analysis of the human tissue.

For the human sections a similar semi-quantitative approach was applied for the lightmicroscopic analysis. Layers of the OB were defined following indications provided in (Mai and Paxinos, 2012).

Subdivisions of basal ganglia (Mai et al., 2008) and brainstem nuclei (Haines, 2011) were identified, using atlases, based upon their topographical position. Supplement Figure S1 illustrates micrographs of sections used for the analysis, taken at the levels of basal ganglia and mesencephalon showing corresponding subdivisions in nuclei and white matter regions. The presence of AD-related neuropathology was estimated semi-quantitatively as - (negative), + (low), ++ (moderate) and +++ (high) based on densities of NFTs, plaques and positive neuronal cells. In addition, qualitative descriptions were made on the distribution of neuropathology and the morphological features within the structures. Specifically, a classification of immunoreactive substrates was made upon the categorization proposed by Duyckaerts et al. (Duyckaerts et al., 2009). Amyloid deposits were identified as neuritic plaques (NPs) when they presented a tau positive corona of dystrophic neurites or as plaques when such neuritic components were absent. tau immunoreactivity was further identified as neuropil threads (NTs) when stained substrates had the form of small, tortuous processes and as NFTs when immunoreactivity also present in neuronal somata. Photographs were made using a dotSlide BX51microscope (Olympus, Japan). 


\section{Results}

The main goals of the present study were to describe the distribution of $\mathrm{UBB}^{+1}$ in the brain of a $\mathrm{UBB}^{+1}$ tg mice model compared to control mice and to compare the distribution to that in homologous human brain areas. One can observe high or intense staining of individual neurons and a low density of intensely stained neurons and vice versa. No differences in immunoreactivity were found among 3-, 7- and 15-month-old 3413 tg mice (Table 2, supplement Table S2) and $\mathrm{UBB}^{+1}$ was absent in all brain regions of the control mice (van Tijn et al., 2011). The incubation of $\operatorname{tg}$ mouse brain tissue with ${ }^{6} \mathrm{His} \operatorname{tag} \mathrm{UBB}^{+1}$ and the preimmune control serum resulted in an absence of immunopositive staining (Irmler et al., 2012). Relative $\mathrm{UBB}^{+1}$ immunoreactivity scores for intensity and density in the mouse brain are shown in Figure 2. Abbreviations of anatomical brain structures are listed in alphabetical order in the list of abbreviations and in supplement Table S1. In the next paragraphs we will discuss first the results of the mouse line 3413 brain anatomy followed by the results of the human immunohistochemical study.

\subsection{Mouse studies}

\subsection{1 $\mathrm{UBB}^{+1}$ in the telencephalon and diencephalon}

The present study extends and provides specific details in follow up to our initial global descriptions of $\mathrm{UBB}^{+1}$ expression in the forebrain (e.g., cerebral cortex, hippocampus, dentate gyrus, amygdala and striatum) and the brainstem in the 3413 tg mice (Fischer et al., 2009; Irmler et al., 2012) (Figure 1-2, Table 2). The CaMKIla promoter mainly results in strong protein expression in forebrain areas (Mayford et al., 1996a). In keeping with this, the expression of $\mathrm{UBB}^{+1}$ protein was strong in the forebrain. In addition, after extensive mapping we observed a wide range of $\mathrm{UBB}^{+1}$ expression in hindbrain regions (e.g., brainstem and cerebellum) where other groups also showed the presence of CamKIla (Mayford et al., 1996b). The analysis of the immunohistochemical expression in the mouse brain is aggregated by anatomical region (i.e., telencephalon, diencephalon, mesencephalon, pons, medulla oblongata and cerebellum). 


\subsubsection{Telencephalic structures in the mouse brain}

In most of the forebrain regions, the 3413 mice showed low to very high expression (+; ++; $+++;++++$ ) of $\mathrm{UBB}^{+1}$ (Table 2, Figure 2). In the neocortex, layers $(\mathrm{L})$ 2, 3, 4, 5 and 6 showed a low to moderate intensity with a moderate $(L 5, L 6)$ to high $(L 2, L 3, L 4)$ density of $U_{B B}{ }^{+1}$ cells while $\mathrm{UBB}^{+1}$ was absent in $\mathrm{L} 1$. These data demonstrated that $\mathrm{UBB}^{+1}$ was not uniformly stained in the classical six layers of the neocortex. The caudomedial entorhinal cortex (CEnt), retrosplenial granular cortex (RSGa, b and c) and primary visual cortex (V1) showed a high UBB+-intensity while the other cortical areas (e.g., APir, Cg1, Cg2, Pir, CxA, DEn, DP, Ect, FrA, IL, LO, M2, PrL, RSD, V2MM, VEn and VO) varied between a low to moderate intensity (Table 2). A moderate density was present in all the cortical areas except for infralimbic cortex (IL) with a low density and the CEnt, ectorhinal cortex (Ect) and $\mathrm{V} 1$ which showed a high density of $\mathrm{UBB}^{+1}$ cells. The mediolateral area of the secondary visual cortex (V2ML) was $\mathrm{UBB}^{+1}$-negative (Table 2).

$\mathrm{UBB}^{+1}$ neuronal immunoreactivity was distributed throughout the hippocampal region with a variable intensity ranging from moderate to high and a high to very high cell-density. However, the molecular layer of the dentate gyrus (Mol), the stratum lacunosummoleculare ( $\mathrm{Lmol}$ ) and the stratum radiatum (Rad) were $\mathrm{UBB}^{+1}$-negative. The polymorph layer (PoDG) of the dentate gyrus showed a moderate $\mathrm{UBB}^{+1}$ intensity and density while the granular layer $(\mathrm{GrDG})$ was highly $\mathrm{UBB}^{+1}$ positive with a very high density. (Table 2 , Figure 2).

In several amygdala nuclei, a low to moderate intensity and a moderate density of UBB ${ }^{+1}$ ir cells was observed. In the anterior cortical amygdaloid nucleus (ACo) little or no $\mathrm{UBB}^{+1}$ immunoreactivity was observed (Table 2). Other telencephalic structures are described in the supplements.

The neuronal cells in $\mathrm{OB}$ and the accessory olfactory bulb (AOB), both receiving olfactory primary afferents, contain a noticeable amount of $\mathrm{UBB}^{+1}$ within a moderate number of positive neurons (Table 2, Figures 1-2). The $\mathrm{OB}$ is subdivided in certain regions which all showed $\mathrm{UBB}^{+1}$ immunoreactivity. Specifically, the glomerular layer $(\mathrm{Gl})$ and external plexiform layer (EPI) of the $\mathrm{OB}$ showed a moderate $\mathrm{UBB}^{+1}$-intensity and a low density. A 
low intensity and density was present in the mitral cell layer (Mi), in the internal plexiform layer (IPI) and in the granule cell layer (GrO) of the OB. The ependymal and subendymal layer/olfactory ventricle $(\mathrm{E} / \mathrm{OV})$ had low intensity $\mathrm{UBB}^{+1}$ immunoreactivity with a moderate density of positive neurons. A moderate intensity and density were observed in the nucleus of the lateral olfactory tract (LOT). In the $A O B$, the mitral (MiA) and glomerular (GIA) cell layer of the $A O B$ expressed a moderate $\cup B B^{+1}$-intensity while a low intensity was detected in the granule cell layer $(\mathrm{GrA})$ of the $\mathrm{AOB}$. All subdivisions of the $\mathrm{AOB}$ showed a moderate density. The anterior olfactory area is subdivided into several anterior olfactory nuclei: dorsal (AOD), external (AOE), lateral (AOL), medial (AOM), posterior (AOP), ventral (AOV). The AOE expressed a moderate $\mathrm{UBB}^{+1}$-intensity while the AOD, AOL, AOM, AOP and AOV showed a low intensity. A moderate density was present for all the different nuclei of the anterior olfactory area.

The basal ganglia structures in the telencephalon as reported previously were also immunoreactive for $\mathrm{UBB}^{+1}$. The neostriatum (caudate nucleus/putamen ( $\mathrm{CPu}$ )) showed a moderate intensity and density of $\mathrm{UBB}^{+1}$-ir cells. A specific subpopulation of large neurons in $\mathrm{CPu}$ is very highly stained for $\mathrm{UBB}^{+1}$ while smaller neurons are rather moderate $\mathrm{UBB}^{+1}$ immunoreactive (Table 2). In addition to the neostriatum, the nucleus accumbens (Acb), the olfactory tubercle ( $\mathrm{Tu}$ ), and the Islands of Calleja (ICj) showed a moderate to high density of $\mathrm{UBB}^{+1}$ positive cells (Table 2, Figures 1-2). The globus pallidus lateralis (LGP) and medialis (MGP) were $\mathrm{UBB}^{+1}$-negative (Table 2). Another basal ganglia component, the subthalamic nucleus (STh) located in diencephalon, showed a low expression for $\mathrm{UBB}^{+1}$. Adjacent to the basal ganglia, the $\mathrm{UBB}^{+1}$ expression was low in the NBM in tg line 3413 with a moderate number of positive cells (Table 2).

\subsubsection{Diencephalic structures in the mouse brain}

A detailed summary of the $\mathrm{UBB}^{+1}$ expression in the diencephalic structures is given in the supplements. 


\subsection{4 $\mathrm{UBB}^{+1}$ in the mesencephalon}

\section{Mesencephalic structures in the mouse brain}

We analyzed the $\mathrm{UBB}^{+1}$ expression in tectal and tegmental mesencephalic brain regions. The IC contained a clear UBB ${ }^{+1}$-immunoreacitiviy. The IC is subdivided into the dorsal cortex (DCIC) and external cortex (ECIC), the central nucleus (CIC) and the nucleus of the brachium of the IC (BIC) which showed staining intensities varying from low to moderate and densities from moderate to high number of $\mathrm{UBB}^{+1}$-ir cells (Table 2, Figures 1-2). The IC is connected with brain structures located in the diencephalon, pons and medulla oblongata and which are all part of the auditory system e.g., medial geniculate nucleus (MG; diecenphalon), the medullary cochlear nuclei (DC, VC; medulla oblongata), the pontine superior olive and the pontine trapezoid nucleus ( $T z$; pons). The subnuclei of the medial geniculate nucleus (dorsal, ventral, medial part and the marginal zone) expressed a low UBB ${ }^{+1}$-intensity and density (Table 2 ). The DC showed a high cell-intensity while the staining in the $\mathrm{VC}$ and superior olive was rather low. No immunoreactiviy was detected in the Tz (Table 2).

Several raphe subnuclei are also located in the mesencephalon: caudal (Cli) and rostral (Rli) linear nuclei of the raphe, raphe cap (RC), median (MnR) and paramedian (PMnR) raphe and all the subnuclei of the dorsal raphe nucleus (DR) namely: caudal part (DRC), dorsal part (DRD), interfascicular part (DRI), ventral part (DRV), ventrolateral part (DRVL). The RC and Cli expressed a low $\mathrm{UBB}^{+1}$ staining while the Rli was negative for $\mathrm{UBB}^{+1}$. A moderate intensity and density was present in the MnR and PMnR. The different subnuclei of the dorsal raphe showed intensities and densities varying from moderate to high (Table 2, Figures 1-2).

Additional mesencephalic structures and their immunoreactivity for $\mathrm{UBB}^{+1}$ are summarized in the supplements. 


\subsection{5 $\mathrm{UBB}^{+1}$ in the pons, cerebellum and medulla oblongata}

\section{Pons and cerebellum structures in the mouse brain}

As outlined in the introduction, previous experiments examined the presence of $\mathrm{UBB}^{+1}$ in respiratory nuclei (Irmler et al., 2012). Table 2 and Figure 2 show a high to very high UBB ${ }^{+1}$ intensity in the nucleus parabrachialis, a pontine respiratory control center. Differences were present in medial external (MPBe), lateral ventral (LPBV) and in lateral internal (LPBI) parabrachial nucleus which have a moderate intensity, and the waist part (PBW), with a low intensity of $\mathrm{UBB}^{+1}$-ir cells. The $\mathrm{UBB}^{+1}$ density in the different parabrachial nuclei varied from moderate to high. The locus coeruleus (LC) exhibited a similar low to moderate staining intensity (Irmler et al., 2012). The ventral part of the nucleus subcoeruleus showed a low $\mathrm{UBB}^{+1}$ expression as well while the dorsal part was negative (supplementary Table S2). The staining in the pontine part of the raphe nuclei (PnR) was moderate for intensity and density (Table 2, Figure 1). A more detailed overview about pontine nuclei and the $\mathrm{UBB}^{+1}$ immunoreactivity is given in the supplements.

The cerebellum showed a high expression of $\mathrm{UBB}^{+1}$ in specific cerebellar regions and will be addressed in a separate study.

\section{Medulla oblongata in the mouse brain}

As reported previously, high $\mathrm{UBB}^{+1}$ expression occurred in brain stem centers namely the nucleus of the solitary tract (Sol), the area postrema (AP) and the dorsal motor nucleus of the vagus nerve (10N) (Irmler et al., 2012) (Table 2 and Figure 2).

With respect to the medullary raphe nuclei, the raphe obscurus (ROb) and the raphe pallidus ( $R P a)$ showed a low $U_{B B}^{+1}$ staining while the raphe magnus (RMg) showed a high intensity and a moderate density (Table 2). More medullary structures positive for $\mathrm{UBB}^{+1}$ are described in the supplements. 
Table 2: $U \mathrm{BB}^{+1}$ immunoreactivity in the cerebral cortex, hippocampus, dentate gyrus, striatum, amygdala, nucleus parabrachialis, locus coeruleus, nucleus of the tractus solitarius, olfactory area, basal ganglia, nucleus basalis of Meynert, auditory area and in the raphe nuclei of $3413 \mathrm{tg}$ mice. Relative $U B B^{+1}$ immunoreactivity scores for intensity and density are illustrated in Figure 2. No UBB ${ }^{+1}$ immunoreactivity was present in any brain regions in the WT mice.

\begin{tabular}{|c|c|c|c|c|c|}
\hline Brain nuclei & $\begin{array}{c}3413 \\
\text { UBB }^{+1} \text { I }\end{array}$ & $\begin{array}{c}3413 \\
\text { UBB }^{+1} \text { D }\end{array}$ & $\begin{array}{l}\text { Brain } \\
\text { nuclei }\end{array}$ & $\begin{array}{c}3413 \\
\text { UBB }^{+1} \text { I }\end{array}$ & $\begin{array}{c}3413 \\
\text { UBB }^{+1} D\end{array}$ \\
\hline \multicolumn{3}{|c|}{ Cerebral cortex } & PrS & ++ & ++ \\
\hline Apir & ++ & ++ & Py & +++ & ++++ \\
\hline Cent & +++ & +++ & Rad & - & - \\
\hline Cg1 & ++ & ++ & $\mathrm{Sb}$ & ++ & ++ \\
\hline Cg2 & ++ & ++ & Shi & ++ & +++ \\
\hline Cortical L1 & - & - & Slu & +++ & ++++ \\
\hline Cortical L2 & ++ & +++ & \multicolumn{3}{|c|}{ Dentate gyrus } \\
\hline Cortical L3 & + & +++ & PoDG & ++ & ++ \\
\hline Cortical L4 & + & +++ & GrDG & +++ & ++++ \\
\hline Cortical L5a & + & + & \multicolumn{3}{|c|}{ Amygdala nuclei } \\
\hline Cortical L5b & ++ & ++ & AAD & + & + \\
\hline Cortical L6 & + & ++ & AAV & + & + \\
\hline CXA & + & ++ & Aco & - & - \\
\hline Den & ++ & ++ & Ahi & + & ++ \\
\hline DP & + & ++ & AhiAL & + & ++ \\
\hline
\end{tabular}




\begin{tabular}{|c|c|c|c|c|c|}
\hline Brain nuclei & $\begin{array}{c}3413 \\
\text { UBB }^{+1} \text { I }\end{array}$ & $\begin{array}{c}3413 \\
\text { UBB }^{+1} \text { D }\end{array}$ & $\begin{array}{l}\text { Brain } \\
\text { nuclei }\end{array}$ & $\begin{array}{c}3413 \\
\mathrm{UBB}^{+1} \text { I }\end{array}$ & $\begin{array}{c}3413 \\
\text { UBB }^{+1} \mathrm{D}\end{array}$ \\
\hline Ect & ++ & +++ & AhiPM & ++ & ++ \\
\hline FrA & ++ & ++ & Astr & ++ & ++ \\
\hline IL & + & + & BLA & + & ++ \\
\hline LO & ++ & ++ & BLP & ++ & ++ \\
\hline M2 & ++ & ++ & BLV & + & ++ \\
\hline Pir & ++ & ++ & BMA & + & ++ \\
\hline PrL & + & ++ & BMP & + & ++ \\
\hline RSD & ++ & ++ & $\mathrm{CeC}$ & ++ & ++ \\
\hline RSGa & +++ & ++ & CeL & ++ & ++ \\
\hline RSGb & +++ & ++ & CeM & + & ++ \\
\hline RSGc & +++ & ++ & CeMAD & + & ++ \\
\hline V1 & +++ & +++ & CeMAV & + & ++ \\
\hline V2MM & ++ & ++ & CeMPV & + & ++ \\
\hline V2ML & - & - & I & ++ & + \\
\hline Ven & ++ & ++ & IM & + & ++ \\
\hline VO & ++ & ++ & La & ++ & ++ \\
\hline \multicolumn{3}{|c|}{ Hippocampus } & LaDL & ++ & ++ \\
\hline CA1 & +++ & ++++ & LaVL & ++ & ++ \\
\hline
\end{tabular}




\begin{tabular}{|c|c|c|c|c|c|}
\hline Brain nuclei & $\begin{array}{c}3413 \\
\mathrm{UBB}^{+1} \text { I }\end{array}$ & $\begin{array}{c}3413 \\
\text { UBB }^{+1} \text { D }\end{array}$ & $\begin{array}{l}\text { Brain } \\
\text { nuclei }\end{array}$ & $\begin{array}{c}3413 \\
\text { UBB }^{+1} \text { I }\end{array}$ & $\begin{array}{c}3413 \\
\text { UBB }^{+1} D\end{array}$ \\
\hline CA2 & +++ & ++++ & LaVM & + & + \\
\hline CA3 & +++ & +++ & MeA & + & ++ \\
\hline FC & ++ & +++ & MeAD & ++ & ++ \\
\hline $\lg$ & ++ & +++ & MeAV & ++ & ++ \\
\hline Lmol & - & - & MePD & ++ & ++ \\
\hline Mol & - & - & MePV & + & ++ \\
\hline PaS & ++ & ++ & PLCo & ++ & ++ \\
\hline
\end{tabular}



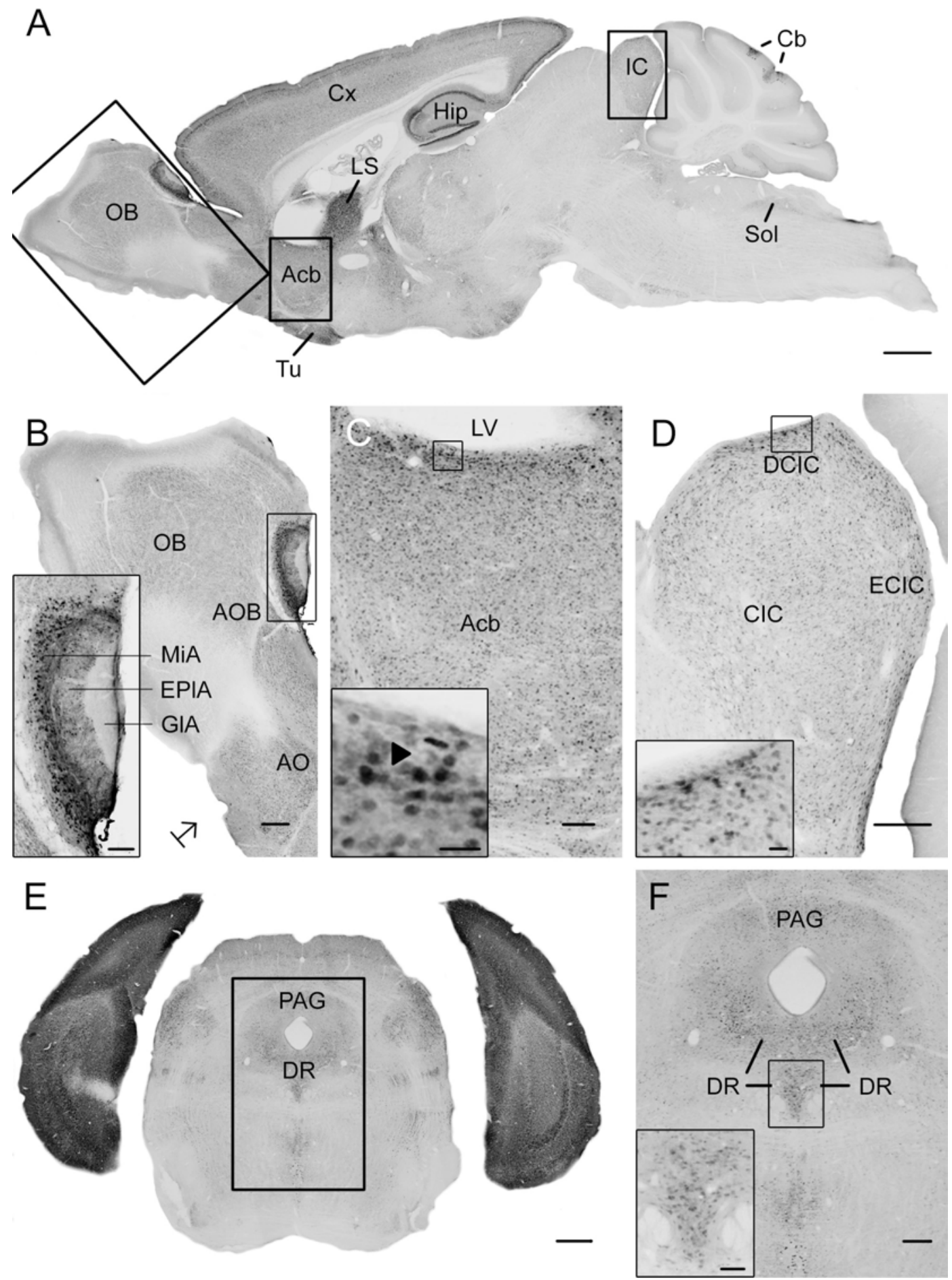
Figure 1: Photomicrographs of $U B B^{+1}$ distribution in the brain of $U_{B B^{+1}} \operatorname{tg}$ mice (line 3413) shown in sagittal and coronal sections. A) Sagittal overview of $U B B^{+1}$ staining in a 7-month-old 3413 tg mouse corresponding to sagittal Figure 106 in the mouse brain atlas of Franklin and Paxinos (Franklin and Paxinos, 2007) with rectangles showing the locations of higher magnifications in (B-D). b) $O B, C)$ $A c b$ and D) IC. B) shows the presence of $U B B^{+1}$ in neurons of the $O B$, accessory olfactory bulb (AOB) and the anterior olfactory area $(A O)$ at higher magnification. The insert shows a higher magnification of the $A O B . C) \cup B B^{+1}$ expression in the Acb. Insert shows higher magnification of $U B B^{+1}$ positive cells. Filled triangle shows a cell in which the $U B B^{+1}$ staining is cytoplasmic and the nucleus is negative. D) $U B B^{+1}$ immunoreactivity in the IC. Insert shows a higher magnification of the immunoreactive cells in the DCIC. Asterisk shows cytoplasmic UBB ${ }^{+1}$ staining. E) Coronal section of a 15-month-old $3413 \mathrm{tg}$ mouse showing the presence of $U B B^{+1}$ in the dorsal raphe (corresponding to coronal Figure 69 in the mouse brain atlas of Franklin and Paxinos (Franklin and Paxinos, 2007)). F) shows this region at a higher magnification. Insert in $(F)$ shows the UBB ${ }^{+1}$-immunoreactive cells in the $D R . A=1 \mathrm{~mm},(B, D, F)=200 \mu \mathrm{m}$, (insert in $B, C)=100 \mu \mathrm{m}, E=500 \mu \mathrm{m}$, (insert in $C$, insert in $D$ )= $20 \mu \mathrm{m}$, insert in $\mathrm{F}=50 \mu \mathrm{m}$. Acb= nucleus accumbens; $A O B=$ accessory olfactory bulb; $A O=$ anterior olfactory area; $C b=$ cerebellum; $C I C=$ central nucleus of $I C ; C x=$ cerebral cortex; $D C I C=$ dorsal cortex of IC; $D R=$ dorsal raphe; EpIA=external plexiform layer of the accessory olfactory bulb, ECIC= external cortex of IC; GIA=glomerular layer of the accessory olfactory bulb; Hip= hippocampus; IC= inferior colliculus; $L S=$ lateral septal nucleus; $L V=$ lateral ventricle; MiA=mitral cell layer of the accessory olfactory bulb; $O B=$ olfactory bulb; $P A G=$ periaqueductal gray; Sol= nucleus of the tractus solitarius; Tu= olfactory tubercle. 
A

Intensity

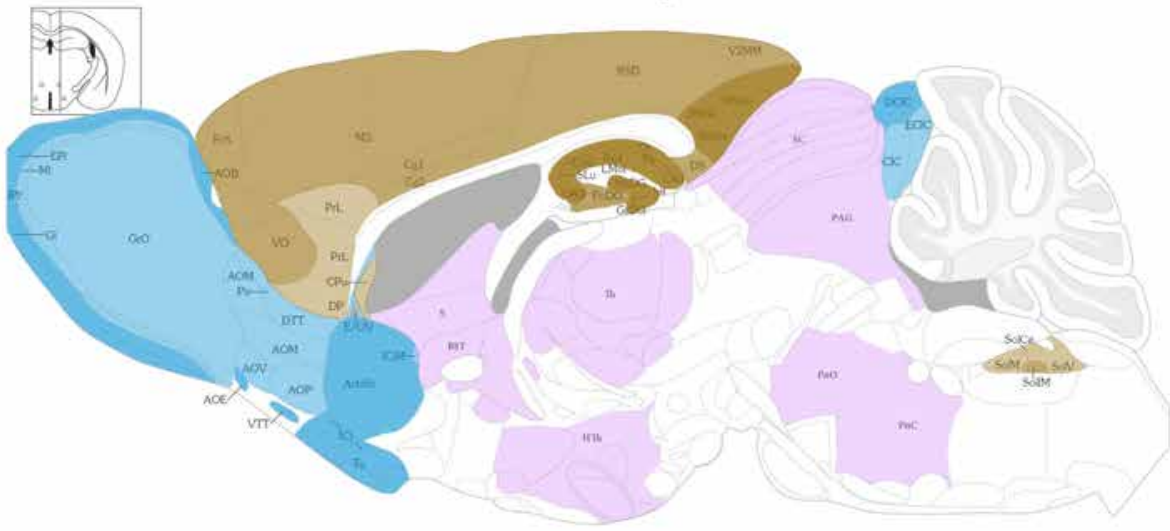

B Density

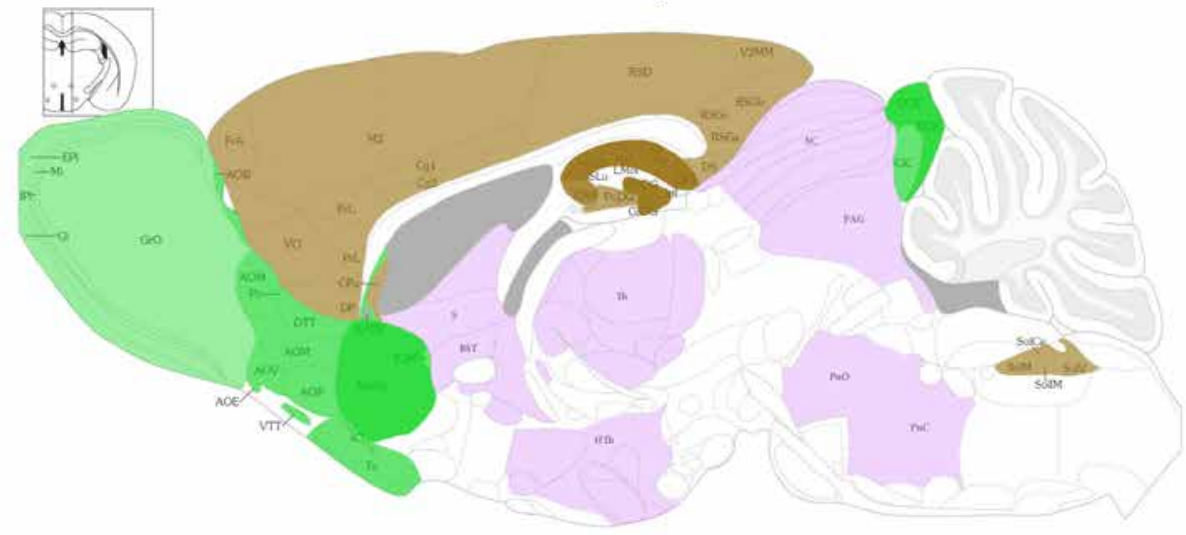

C Intensity | Density

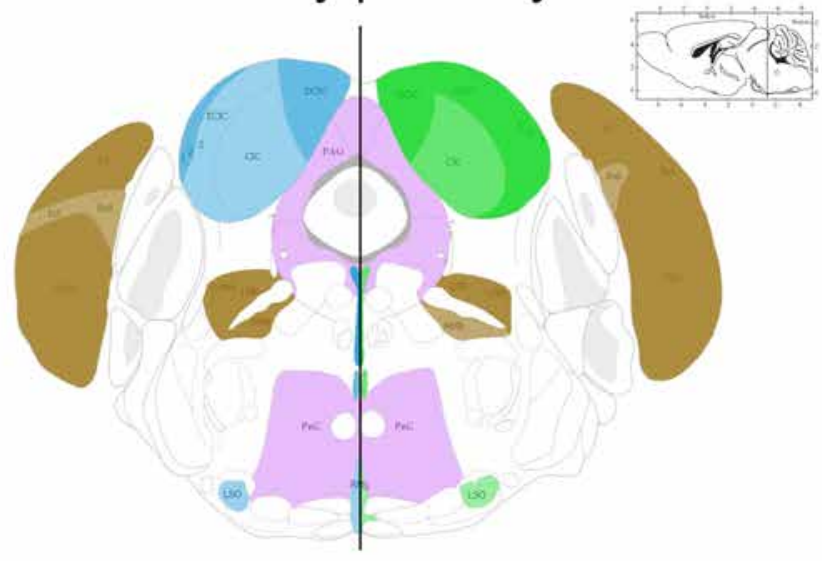


Figure 2: Schematic sagittal $(A, B)$ and coronal $(C)$ overviews showing the mean $U B B^{+1}$ intensity (blue) ( $A, C$ left) and density (green) (B, C right) in a wide range of mouse brain nuclei. Regions previously reported to be $U B B^{+1}$ immunoreactive (cerebral cortex, hippocampus, dentate gyrus, amygdala, striatum, nucleus parabrachialis and Sol) are shown in brown. The UBB ${ }^{+1}$ intensity and density in $\mathrm{OB}, A c b, I C$ and the raphe nuclei are shown in blue and green respectively. Different gradations of brown, blue and green are used indicating the level of staining intensity or density with a light gradation for a low intensity or density, a medium gradation for a moderate intensity or density level, a dark gradation for a high intensity or density level and a very dark gradation for a very high intensity or density. Other sizeable regions with a low to moderate $U B B^{+1}$ immunoreactivity are shown in purple. However, more regions are positive for $U_{B B}^{+1}$, for details see Supplementary Table 2. Figures are adapted from the mouse brain atlas of Franklin and Paxinos Figure 106 and 73 respectively (Franklin and Paxinos, 2007). BST= bed nucleus of the stria terminalis; Gi= gigantocellular reticular nucleus; $H$ Th= hypothalamus; $P A G=$ periaqueductal gray; $P n C=$ pontine reticular nucleus, caudal part; $\mathrm{PnO}=$ pontine reticular nucleus, oral part $\mathrm{S}=$ septal area; $\mathrm{SC}=$ superior colliculus; $T h=$ thalamus

\subsection{Human studies}

\subsection{1 $\mathrm{UBB}^{+1}$ in the human olfactory area, basal ganglia and nucleus basalis of Meynert}

The olfactory areas analyzed comprised olfactory bulb, olfactory tract and the cerebral cortical area adjacent to the bulb and tract, piriform cortex (Pir). In order to investigate whether the distribution of $\mathrm{UBB}^{+1}$ immunoreactivity overlaps with other pathological changes typical of $A D$, these areas were also mapped using antibodies against neuronal markers for pre-tangles, NFTs and plaques. These immunohistochemical results showed that $A D$ markers (NFTs and plaques) were present in all $A D$ cases, but not in the nondemented controls (Table 3, Figure 3-4). A clear differential distribution of pathology was detected within the olfactory system of the Braak 5 cases. The Pir showed high density of immunoreactive substrates (Figure 4), which progressively decreased in the olfactory tract and the bulb. Specifically, the olfactory tract expressed moderate AD-related pathology, and immunoreactivity was restricted to island in the central portion of the structure. This region has been identified by Del Tredici et al. as the anterior olfactory nucleus (AON) 
(Figure 3) (Del Tredici et al., 2002). In the OB, either no pathology or very few reactive substrates were present. The Pir exhibited both $A \beta$ deposition and tau pathology (Figure 4). High densities of plaques, both neuritic and non-neuritic, were homogeneously distributed throughout the layers of the Pir. NFTs and NTs were present in moderate to high densities and showed a fairly consistent laminar distribution, being concentrated in middle and deep layers.

Staining and analysis were also performed on cryoprotected olfactory tissue sections (Table 1, Figure 3). In these samples, the anatomical subdivisions of the human OB were not always clear to distinguish. In the external part of the bulb, the sections presented a layer recognizable as the olfactory nerve layer. Adjacent regions, most likely the glomerular layer, exhibited rounded structures organized in a line over the surface of the bulb. More internally, a layer composed of fibers, most likely the external plexiform layer was distinguishable. In the central portions of these sections, a high number of cells could be visualized. Presumably, these regions comprised the mitral cell, the internal plexiform, and the granule cell layers. Immunohistochemical expression of $\mathrm{UBB}^{+1}$ and $\mathrm{AD}$-specific neuropathology in the $\mathrm{OB}$ and olfactory tract (more specifically the $A O N$ ) is illustrated in Figure 3. $\mathrm{UBB}^{+1}$ immunoreactivity was mainly restricted to central portions of the bulb, external layers exhibiting either no pathology or few isolated cells and stained threads. All three AD cases (cryoprotected tissue) expressed low to high density of NTs and NFTs throughout the layers of the bulb and expressed few isolated $\mathrm{UBB}^{+1}$ positive cells in the central regions. Only one case (patient \#10) showed $A \beta$ deposition, which was restricted to the central regions of the bulb namely the granule cell layer. 
4

?

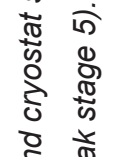

穴

这

$\frac{\sqrt{\pi}}{\pi} \frac{\pi}{\pi}$

$\stackrel{2}{\Omega}$

ลิ ণ

है ?

ఏ ल

$\begin{array}{ll}0 & \\ 2 & 0 \\ 0 & 0 \\ \frac{\pi}{2} & 0 \\ \frac{1}{2} & \frac{\pi}{4}\end{array}$

क क क

ल

仓

क व

बह

永

$\therefore$

ป ह

$\frac{\pi}{2}$

$\begin{array}{cc}\frac{\pi}{d} & 0 \\ \frac{0}{0} & 0 \\ \frac{0}{0} & \frac{\pi}{4}\end{array}$

$\frac{\pi}{2} \frac{\pi}{2}$

齐 选

(1) 包

苟高

गิ

ఫे

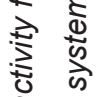

ฮั ते

ફ

ले है

क

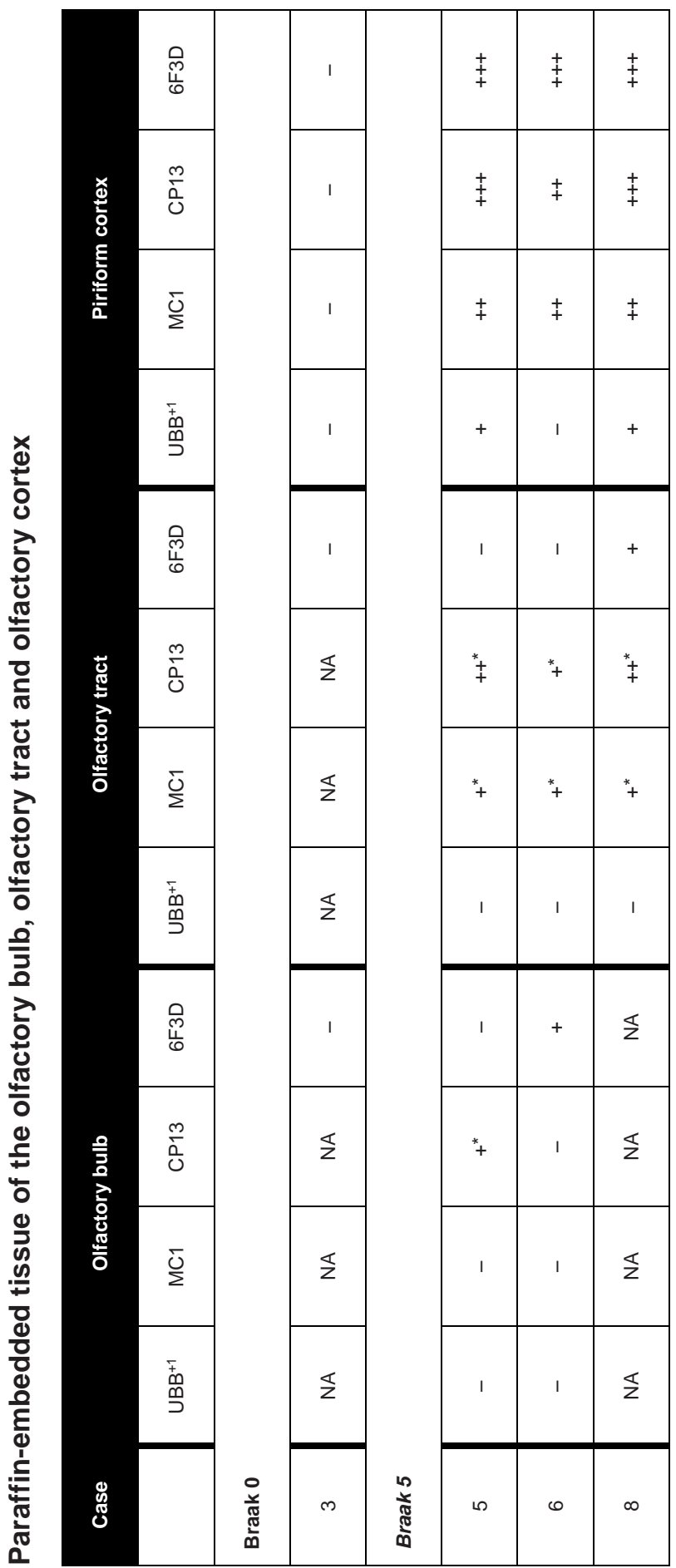




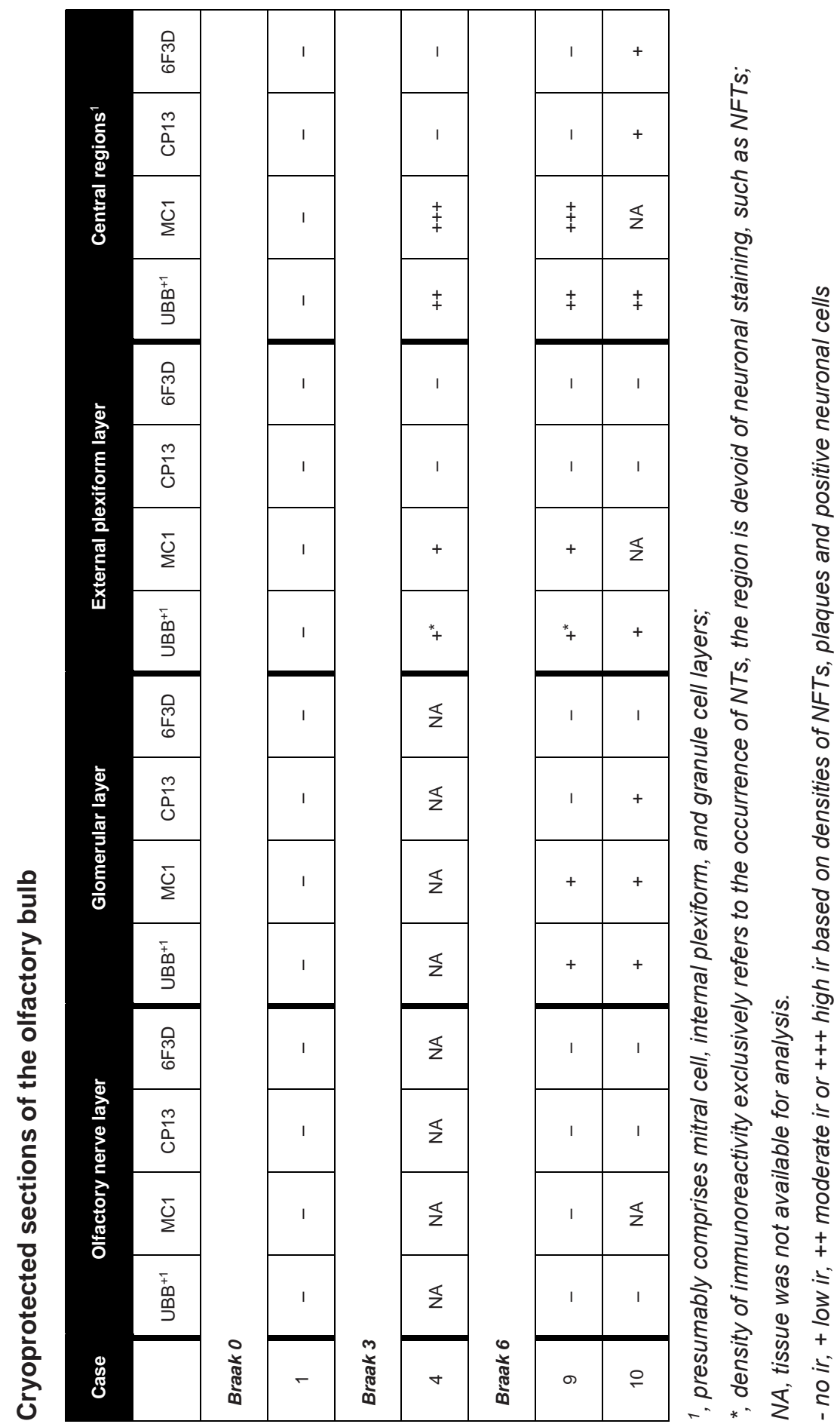



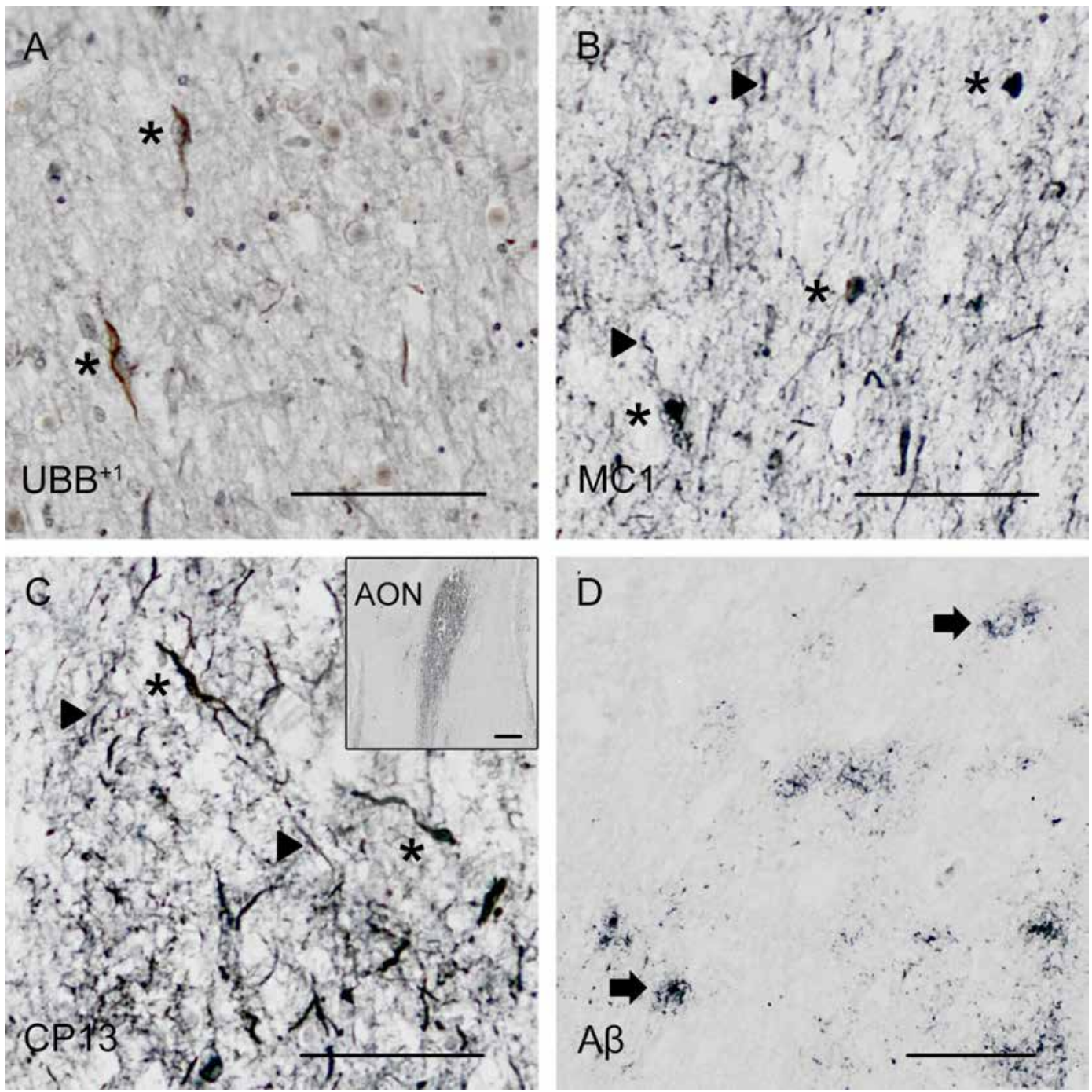

Figure 3: Expression of $U B B^{+1}$ and $A D$ neuropathology (NFTs, NTs and $A \beta$ ) in sections of the human olfactory bulb and tract of patient \#10 (Braak 6). A-D) shows olfactory tissue incubated with antibodies against $\left.A) U B B^{+1}, B\right)$ pre-tangles (MC1), C) tangles (CP13) and $\left.D\right) A \beta$ plaques. Asterisk shows intracellular accumulations, represented by neuronal staining A) $U B B^{+1}$ immunoreactivity present in neuronal cells of the OB (asterisk). B-C) Presence of misfolded tau in pretangles and NFTs (asterisk). NTs are shown by the filled triangles. C) The insert shows the AON of the tract. D) Presence of $A \beta$ plaque formation (arrow). $(A-D)=100 \mu \mathrm{m}$, insert in $C=500 \mu \mathrm{m}$. AON= anterior olfactory nucleus. 

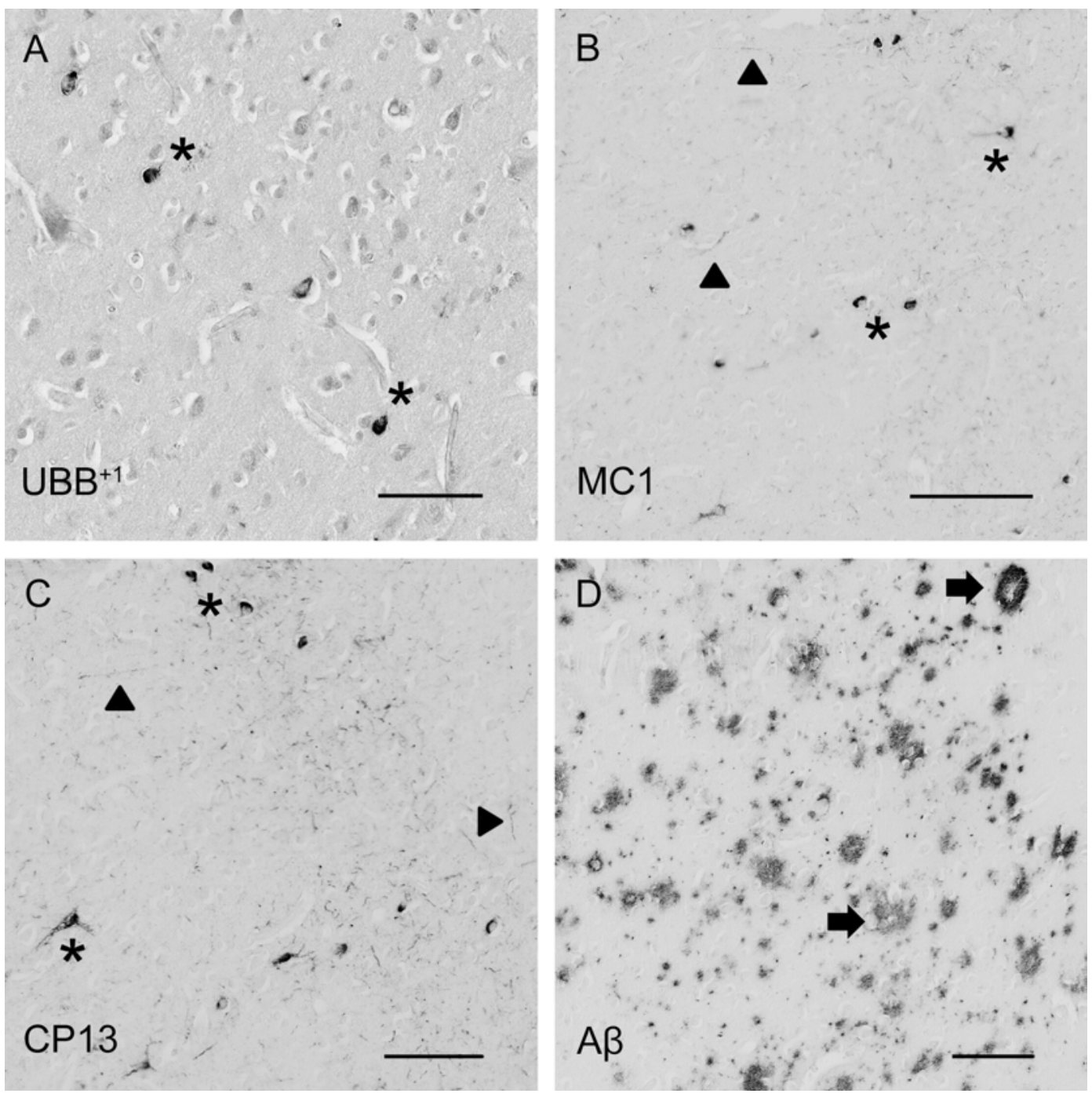

Figure 4: Photomicrographs of $U B B^{+1}$ and $A D$ neuropathology (NFTs, NTs and $A \beta$ plaques) in the human piriform (Pir) cortex adjacent to the OB of patient \#5 (Braak 5). A-D) shows the olfactory tissue incubated with antibodies against $\left.A) U B B^{+1}, B\right)$ pre-tangles (MC1), C) tangles (CP13), and D) $A \beta$ plaques. Asterisk shows intracellular accumulations, represented by neuronal staining. A) The presence of $\mathrm{UBB}^{+1}$ in the neuronal cells (asterisk) of the Pir. B-C) Pir expresses misfolded tau in pretangles and NFTs (asterisk). NTs are shown by filled triangles. D) Abundant present of A $\beta$ plaque formation (arrow) in the Pir. $(A-D)=100 \mu m$. 
With respect to the human basal ganglia including the putamen $(\mathrm{Pu})$ and caudate nucleus (Cd) (forming the neostriatum), globus pallidus (GP) and adjacent regions, such as the Acb and the NBM. In all AD cases, AD-related pathology (plaques and NFTs) was present in the basal ganglia (Table 4). The Braak 3 and the four Braak 5 cases exhibited comparable densities of depositions in the basal ganglia nuclei and in the NBM. The non-demented controls displayed occasional NFTs and $\mathrm{UBB}^{+1}$ positive cells in the NBM, whereas the nuclei of the basal ganglia of the same cases were not affected. Immunohistochemical expression of $\mathrm{UBB}^{+1}$ and $\mathrm{AD}$-specific neuropathology in the $\mathrm{Acb}$ and in the NBM is illustrated in Table 4 and in Figures 5-6.

There was a differential distribution of AD-related pathology within several structures. In the putamen, a rostro-caudal gradient could be detected with respect to $A \beta$ deposition, meaning that rostral portions of the nucleus exhibited a greater density of plaques than caudal portions. Furthermore, NFTs were homogeneously distributed in the rostral Pu, whereas the caudal Pu displayed higher concentrations of tau pathology in the ventral area (PuV) and in the regions adjacent to lateral medullary lamina ( $(\mathrm{ml})$, compared to central portions. The $\mathrm{Cd}$ expressed a homogeneous distribution of plaques, and a concentration of occasional NFTs adjacent to the internal capsule (ic). Comparable with the Cd, the Acb displayed homogenous densities of plaques and occasional NFTs among its subdivisions: subventricular (AcSV), centromedial (AcCM), medial (AcM) and centrolateral (AcCL) regions. The caudate fundus ( $\mathrm{FCd}$ ) and putaminal fundus ( $\mathrm{FPu})$, together with Acb part of the ventral striatum, showed plaques and ocassionally some NFTs. The internal (GPi) and external (GPe) divisions of the GP exhibited, in a few cases, occasional NFTs and NTs that were restricted to areas adjacent to the medial medullary lamina ( $\mathrm{mml}$ ) and the Iml. In contrast, central areas of the nucleus were not affected.

Several structures of the basal ganglia showed selective labeling with either amyloid deposition or tau pathology. Acb, $\mathrm{Cd}$, and Pu expressed severe amyloid pathology, of both the neuritic and non-neuritic type, but showed only few NFTs and NTs. On the other hand, the NBM was significantly affected by both amyloid depositions and tau pathology. GPe and GPi did not exhibit plaques, and were only rarely affected by NFTs and NTs. 
$\mathrm{UBB}^{+1}$ immunoreactivity was not found in the GP. But the $\mathrm{Cd}$ and Pu did express low densities of $\mathrm{UBB}^{+1}$ in regions also affected by tau pathology, including FCd, FPu, PuV, and the areas along $\mathrm{Iml}$ and ic. $\mathrm{UBB}^{+1}$ was present in low levels in the Acb of only one Braak 5 patient (\#5) which was also affected by tau pathology. The NBM exhibited a moderate density of immunoreactivity, which appeared to selectively target cholinergic cells of the nucleus. 


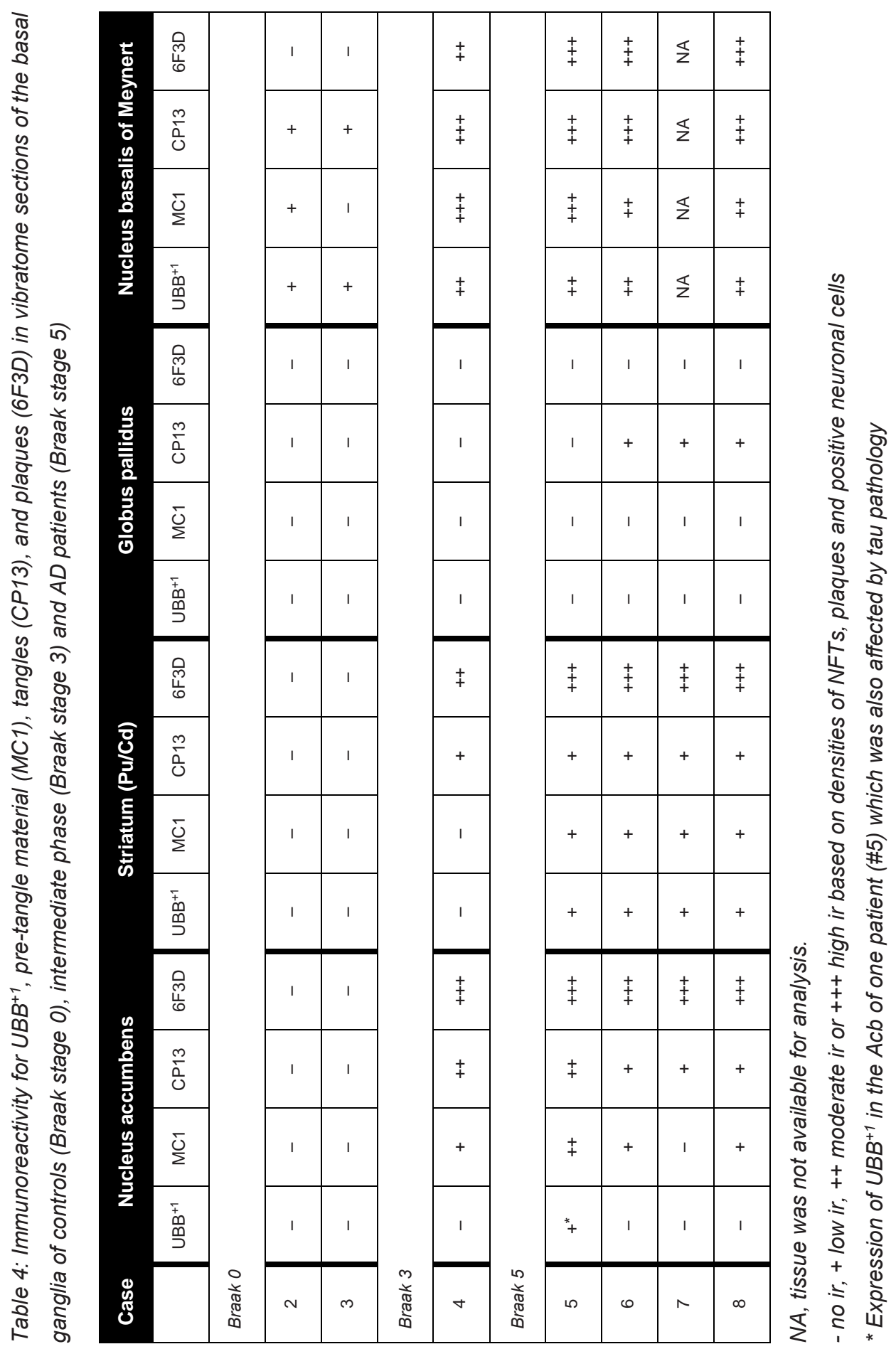




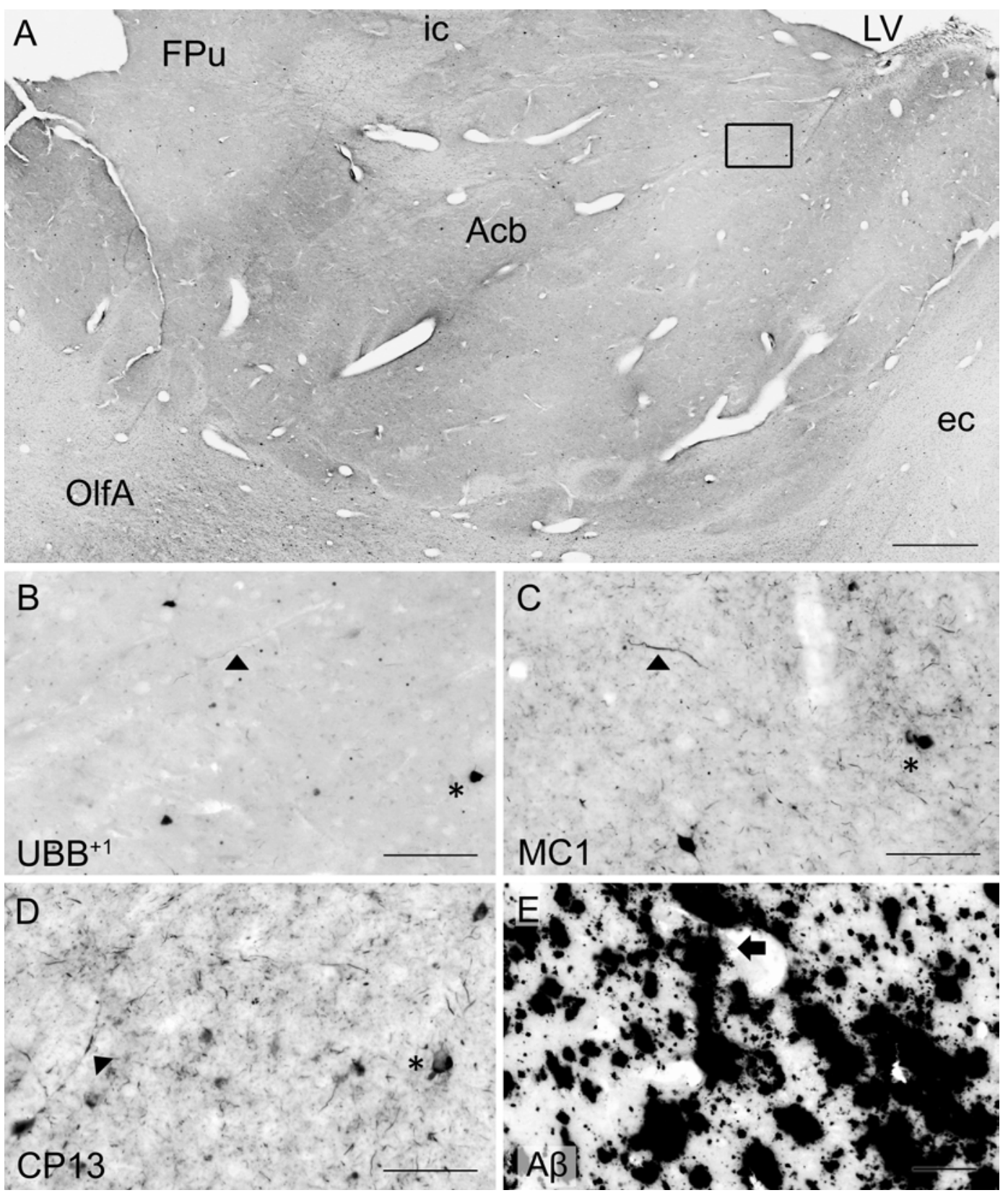

Figure 5: $U B B^{+1}$ and $A D$ neuropathology (NFTs, NTs and $A \beta$ plaques) in the human basal ganglia. A) Photomicrograph of a $50 \mu$ m-thick coronal section from the Acb of patient \#5 (Braak 5). B) Higher magnification of the boxed region in (a) incubated with the antibody against UBB ${ }^{+1}$, C-E) Higher 
magnifications of boxed area in adjacent sections stained for c) pre-tangles material (MC1), D) tangles (CP13) and e) A $\beta$ plaques. The Acb, together with other nuclei of the basal ganglia (Cd and $\mathrm{Pu}$ ), presents little $U \mathrm{UB}^{+1}$ immunoreactivity (asterisk). Asterisks show neurons with intracellular accumulations. NTs are shown by filled triangles and $A \beta$ plaques by an arrow. $A=1 \mathrm{~mm},(B-E)=$ 200 $\mu \mathrm{m}$. Acb= nucleus accumbens, ec= external capsule; FPu= nucleus accumbens putaminal fundus; ic= internal capsule; $L V=$ lateral ventricle; OlfA= olfactory area.
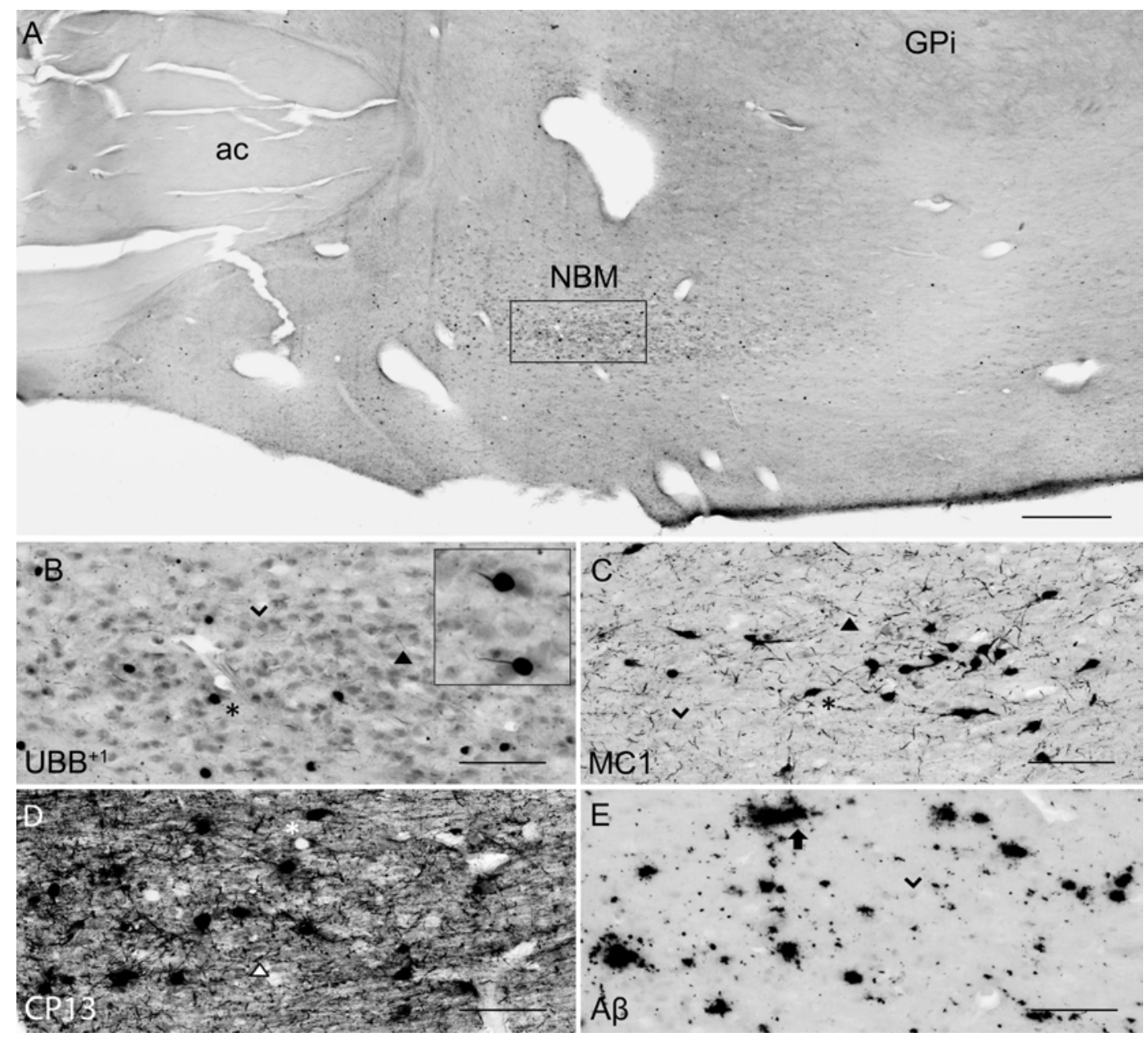

Figure 6: $U B B^{+1}$ and $A D$ neuropathology (NFTs, NTs and $A \beta$ plaques) in the human NBM. A) Representative photomicrograph of a $50 \mu$ m-thick coronal section from the NBM of patient \#4 (Braak 3). B-E) Higher magnifications of the boxed region in (A) incubated with four antibodies against $B$ ) $\left.U_{B B}^{+1}, C\right)$ pre-tangles material $\left.(M C 1), D\right)$ tangles (CP13) and E) A $\beta$ plaques. The cholinergic cells of 
the NBM are shown by an arrowhead. The nucleus is significantly affected by both tau pathology (neuronal staining (asterisk) and NTs (filled triangle)) and extracellular A $\beta$ accumulation (arrow). $U B B^{+1}$ expression (asterisk) is found in cholinergic cells of the NBM. $A=1 \mathrm{~mm},(B-E)=200 \mu \mathrm{m} . a c=$ anterior commissure; GPi= globus pallidus internal segment; NBM= nucleus basalis of Meynert.

\subsubsection{The inferior colliculus and raphe nuclei of the human brain}

In the human tissue, plaques, NFTs and $\mathrm{UBB}^{+1}$ immunoreactive cells were present in the brainstem nuclei of all AD cases (Table 5, (Irmler et al., 2012)). These nuclei presented a differential distribution of pathology among their subdivisions.

In the IC, specifically the CIC was severely affected with plaques in $100 \%$ of AD cases, independent of Braak stage. By contrast, the DCIC and the ECIC subnuclei were relatively spared. The inferior colliculi showed selective vulnerability to $A \beta$ accumulation, expressing a high number of both neuritic and non-neuritic plaques (Table 5, Figure 7). However, neuronal expression of (pre)-tangles and $\mathrm{UBB}^{+1}$ immunoreactive substrates was not present in these regions (Table 5, Figure 7). 


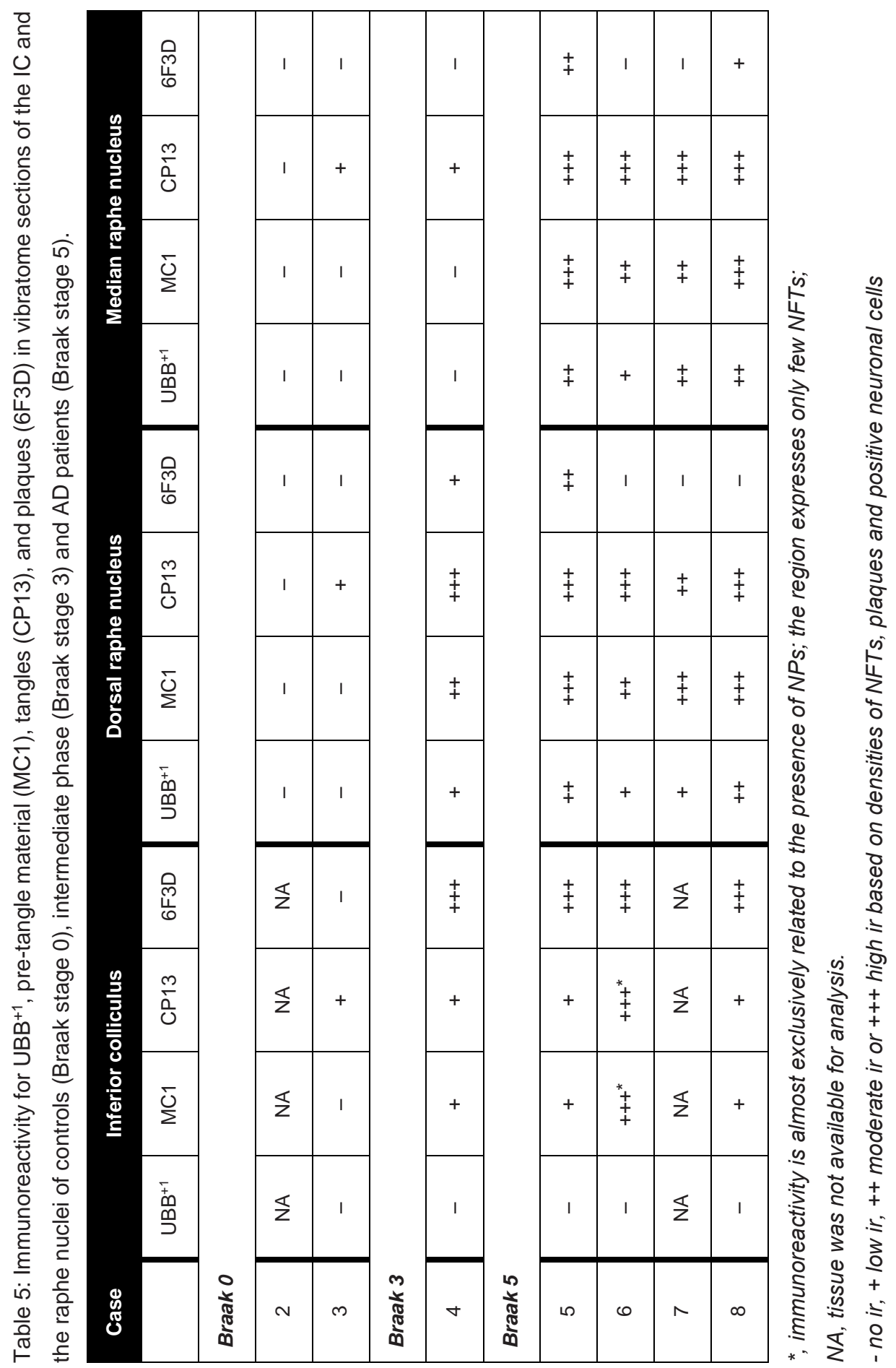




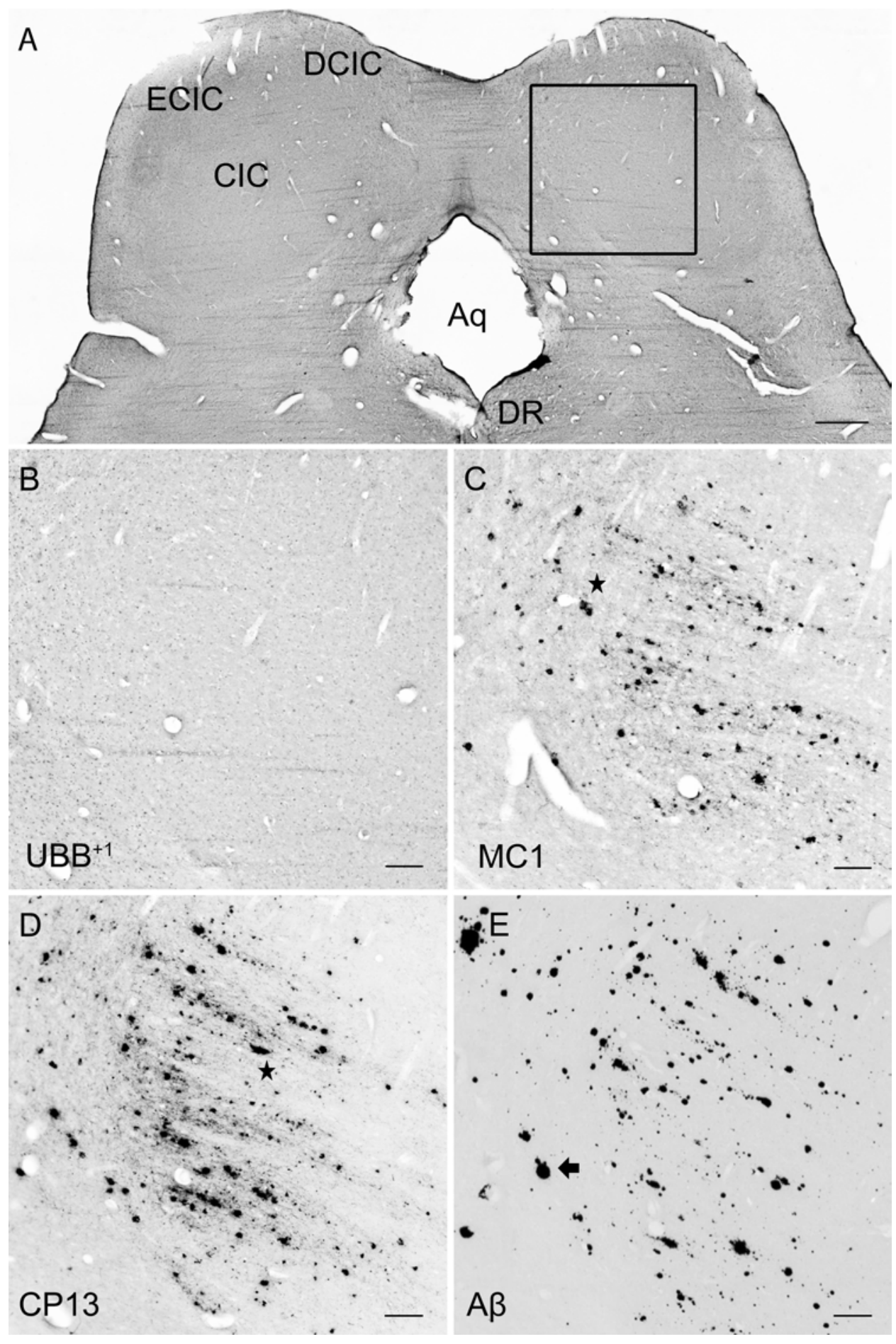


Figure 7: $U B B^{+1}$ and $A D$ neuropathology (NFTs, NTs and $A \beta$ plaques) in the human IC. A) Representative photomicrograph of a $50 \mu$ m-thick coronal section from the brainstem of patient \#6 (Braak 5). B-E) Higher magnifications of the boxed region in (A) incubated with four antibodies against B) $\left.U B B^{+1}, C\right)$ pre-tangles material (MC1), D) tangles (CP13) and E) $A \beta$ plaques. The IC show selective vulnerability to $A \beta$ accumulation, expressing a high number of both neuritic (star) and nonneuritic (arrow) plaques. Neuronal staining, represented by NFTs and UBB ${ }^{+1}$-immunoreactive substrates, is not present in this region. $A=1 \mathrm{~mm},(B-I)=200 \mu \mathrm{m} . A q=$ cerebral aqueduct; $C I C=$ central nucleus of $I C ; D C I C=$ dorsal cortex of $I C ; D R=$ dorsal raphe nucleus; $E C I C=$ external cortex of $I C$; $I C=$ inferior colliculus.

The human raphe tissue showed that the non-demented controls exhibited either no pathology or few NFTs and NTs and did not exhibit plaques or $\mathrm{UBB}^{+1}$ positive cells. The Braak 3 patient expressed less pathology than the four Braak 5 patients, particularly in the MnR. The MnR and DR express selective vulnerability to tau pathology, being almost devoid of plaques. Both MnR and DR were strongly stained by antibodies against pretangles (MC1) and NFTs (CP13) and presented a moderate number of $\mathrm{UBB}^{+1}$-positive structures.

The subdivisions of the DR, namely dorsal DRD, DRV, DRVL, and DRI were equally affected by AD-related pathology with a moderate number of $\mathrm{UBB}^{+1}$ positive structures and high number of tangles. In the MnR, immunoreactive substrates, such as NFTs and NTs, were especially concentrated in the medial division ( $\mathrm{mMnR}$ ), while the PMnR exhibited ADrelated pathology to a lesser extent (only expressing NTs, but not NFTs). Results are summarized in Table 5 and Figure 8. 


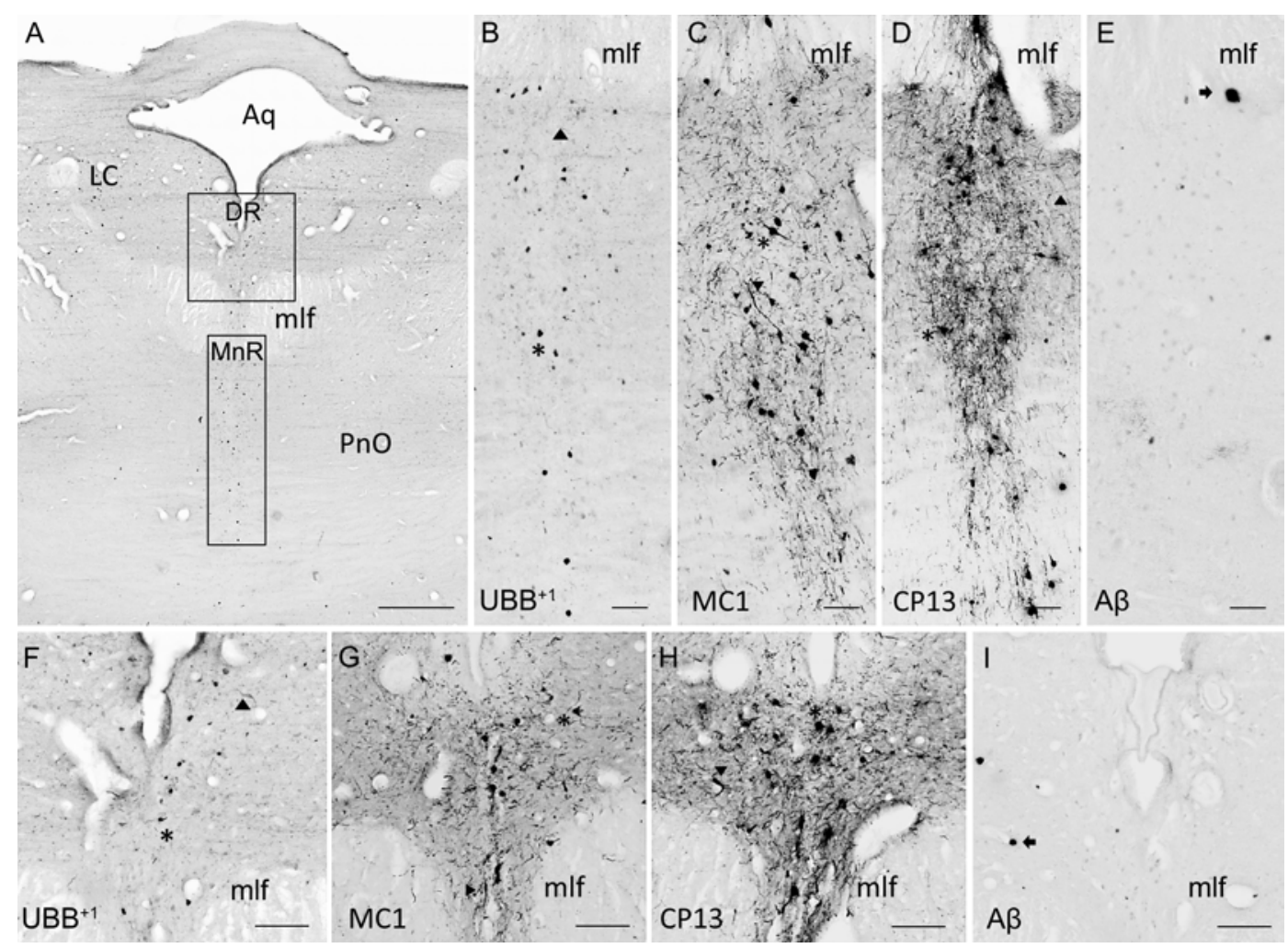

Figure 8: $U B B^{+1}$ and $A D$ neuropathology (NFTs, NTs and $A \beta$ plaques) in the human raphe nuclei. A) Photomicrograph of a $50 \mu$ m-thick section from the brainstem cut perpendicular to the long axis of the spinal cord of patient \#8 (Braak 5). B-E) Higher magnifications of the MnR (lower boxed region in (A)) incubated with four antibodies against $\left.B) U B B^{+1}, C\right)$ pre-tangles material $(M C 1)$, D) tangles (CP13) and E) A $\beta$ plaques. F-I) Larger magnifications of the $D R$ (upper boxed region in (A)) incubated with the same four antibodies. F) Ubi2A; g) MC1; H) CP13; I) 6F3D. The raphe nuclei located in the rostral brainstem (MnR and DR) express selective vulnerability to tau pathology (asterisk=neuronal staining and filled triangle $=N T s$ ), being almost devoid of plaques (arrow). Both MnR and DR are strongly stained by antibodies directed against pre-tangle material (MC1) and NFTs (CP13), and present a moderate number of $U B B^{+1}$ positive substrates (asterisk). $A=1 \mathrm{~mm},(B-I)=200 \mu \mathrm{m}$. $A q=$ cerebral aqueduct; $D R=$ dorsal raphe nucleus, $L C=$ locus coeruleus; $m / f=$ medal longitudinal fasciculus; $M n R=$ median raphe nucleus, $\mathrm{PnO}=$ pontine reticular nucleus oral part. 


\section{Discussion}

The present study describes an extensive topographic mapping of the distribution of $\mathrm{UBB}^{+1}$ in the brain of 3413 mice using a sensitive (i.e., $A B C$ technique) immunohistochemical method (Fischer et al., 2009; Irmler et al., 2012). As a next step, the significance of UBB+1 and neuropathological markers in five brain areas in the mouse brain was compared with the distribution in human postmortem AD brain tissue. Currently, it is not known in either mouse or human whether the distribution of $\mathrm{UBB}^{+1}$ indicates that $\mathrm{UBB}^{+1}$ affects the phenotype everywhere it is present. However, neuropathological studies provide possible links with several additional brain regions, AD phenotype and/or proteasomal dysfunction as discussed below. This was already described in our earlier study (Irmler et al., 2012) for the brainstem region where $\mathrm{UBB}^{+1}$ was found in respiratory centres in the mouse brain which could also be translated to the human brain. Although the value of transgenic models must not be overestimated, our anatomical and functional study in the brainstem of line 3413 showed predictive power for AD (Irmler et al., 2012). Therefore, the presence of $\mathrm{UBB}^{+1}$ can be a useful indicator in other neurodegenerative diseases as shown recently for ER-associated degradation (ERAD) dysfunction in familial encephalopathy with neuroserpin inclusion bodies (FENIB) (Schipanski et al., 2014).

In the next paragraphs, we will discuss the staining results in mouse and human tissue per region, the comparison between the two species and possible links with other neuropathological studies

\subsection{Olfactory bulb and olfactory cortex}

Aging is associated with a decrement in olfactory function, represented by a progressive decline in the ability to detect, identify, and discriminate odors (Mobley et al., 2014). Olfactory deficits have been also described in a variety of neurodegenerative disorders, including AD (Attems et al., 2014). Dysfunction in olfaction is a predictor of the incidence of mild cognitive impairment $(\mathrm{MCl})$ and of the conversion of $\mathrm{MCl}$ to $\mathrm{AD}$ (Djordjevic et al., 2008). 
As mentioned above, the $\mathrm{UBB}^{+1} \operatorname{tg}$ mouse line 3413 accumulates aberrant ubiquitin in low to moderate densities in the $\mathrm{OB}$ and the $\mathrm{AOB}$. In contrast, in humans the olfactory structure, which lacks $A O B$, is relatively spared. Thus $U_{B B}^{+1}$ seems not to be involved in functional impairment of neural cells at lower levels of the processing pathway. $\mathrm{UBB}^{+1}$ positive cells, however, were known to accumulate in areas (e.g., hippocampus and frontal cortex) that are targeted by the neurons of the primary olfactory cortex (Fischer et al., 2009). Therefore, it can be speculated that occurrence of $\mathrm{UBB}^{+1}$ positive cells might rather be implicated in the emergence of symptoms associated with higher-order processing, such as inability to identify or discriminate among odors.

Another focus point is the possible association between noradrenergic deficiency and olfactory dysfunction in AD. Tg2576 mice showed olfactory dysfunction together with degeneration of noradrenergic neurons in the LC, the main area of noradrenaline production (Guerin et al., 2009) and projections to the OB. Additionally, LC degeneration in APP23 tg mice enhances inflammation, amyloid plaque load and neuronal cell death in LC projection regions (e.g., OB) resulting in reduced neuronal integrity and cognitive performance (Heneka et al., 2006). Furthermore, it was demonstrated in an AD mouse model (APPswe, PSEN1dE9) mice that induction of LC degeneration induced exacerbation of olfactory short term memory deficits, a decline in olfactory discrimination and an increase in $A \beta$ load in the granule cell layer of the OB (Rey et al., 2012). The LC is one of the brainstem nuclei wherein tau pathology is apparent, prior to the occurrence of cortical $\beta$ amyloid pathology (Braak et al., 2011). Notably, UBB ${ }^{+1}$ immunoreactivy was also present in the LC (Irmler et al., 2012). Consequently, UBB+1 accumulation might be involved in the noradrenergic neurodegeneration in the LC and olfactory dysfunction in AD. Therefore, performing odor tests with line 3413 would provide valuable information to the question whether $\mathrm{UBB}^{+1}$ accumulation in $\mathrm{OB}$ and $\mathrm{LC}$ is reflected in an impaired olfactory dysfunction. $\mathrm{UBB}^{+1}$ in the $\mathrm{AOB}$ could be associated with and could be tested for social and reproductive behaviors.

With respect to neuropathology, the present study also investigated the distribution of amyloid $\beta$ and tau in the human olfactory bulb, tract and primary olfactory cortex of $A D$ patients and controls. The human olfactory cortex expressed both NFTs and A $\beta$ plaques, 
whereas olfactory bulb and tract more commonly exhibit cytoskeletal pathology. Low densities of NFTs and NTs were found in external layers of the bulb, namely glomerular and external plexiform layers. Higher densities were instead detected in central areas comprising mitral cell, internal plexiform and granule cell layers. In the olfactory tract immunoreactivity was restricted to the AON. Consistent with the results of the present study, Braak et al. found accumulation of NFTs, NTs and plaques in this nucleus (Ohm and Braak, 1987).

In conclusion, the olfactory region is relatively spared in humans for $\mathrm{UBB}^{+1}$ expression and therefore there is no one to one relationship between mouse and human for this region. The present study confirmed the presence of plaques and tangles in the human olfactory region.

\subsection{Basal ganglia}

The different subdivisions of the Acb, involved in reward and reinforcement mechanisms and in regulating emotional behavior (de Jong et al., 2011), showed in the 3413 tg line a high density of $\mathrm{UBB}^{+1}$ immunoreactive cells and a moderate $\mathrm{UBB}^{+1}$ cell-intensity.

By contrast, in the human brain $\mathrm{UBB}^{+1}$ was present only in few isolated cells of $\mathrm{Acb}$ in a single $A D$ case. In all $A D$ patients, Acb showed severe $A \beta$ pathology in both neuritic and non-neuritic plaques, however only a few NFTs and NTs were present. Comparably, senile plaques were demonstrated in the ventral striatum of AD patients (Suenaga et al., 1990). A number of studies have shown dopaminergic (DAergic) dysfunction in AD patients, mainly in the striatum. As the DAergic brain reward system is essential to experience motivation and pleasure, DAergic dysfunction in $A D$ patients was well correlated with apathy defined as a lack of motivation. Apathetic symptoms are detected in up to $47 \%$ of patients with mild $A D$ and up to $80 \%$ in those with severe AD (Mitchell et al., 2011). A reduction in dopamine uptake was shown in the Acb in $A D$ patients compared to nondemented controls (Murray et al., 1995).

These data suggest that there is no relation between mouse and human on $\mathrm{UBB}^{+1}$ expression in the Acb. Apparently, proteasomal malfunctioning seems not to be involved in the DAergic dysfunction seen in AD. 
Additionally to the $\mathrm{Acb}$, the mouse $\mathrm{Pu}$ and $\mathrm{Cd}$ are more affected by $\mathrm{UBB}^{+1}$ accumulation compared to the human brain while the GP was spared of any UBB ${ }^{+1}$ labeling for both mouse and human. We also demonstrated that the human $\mathrm{Pu}$ and $\mathrm{Cd}$ were severely affected by amyloid deposition and being almost devoid of cytoskeletal aggregates, whereas GP was relatively spared of any pathology. This is consistent with previous work demonstrating diffuse plaques in $\mathrm{Cd}$, Acb and Pu combined with an absence of plaques in GP (Brilliant et al., 1997). Taken together, these data suggest that NFTs, as well as UBB ${ }^{+1}$, seem not to be involved in the pathological changes that affect this part of the human basal forebrain. However, we confirmed the presence of plaques in basal ganglia.

The analysis of the human basal ganglia availed an examination of structures that lie adjacent to them. The NBM is considered one of the structures most susceptible to neurofibrillary degeneration, (Mesulam et al., 2004). Atrophy was shown via MRI in the NBM of AD patients, most pronounced in the posterior subdivision (Ch4p) as well as a reduction in the number of $\mathrm{Ch} 4 \mathrm{p}$ cholinergic neurons ranging about $70 \%$ of age-matched controls (Jellinger, 2014). The present study demonstrated severe accumulation of tanglebearing neurons in the NBM of all AD cases, independently of disease progression. The occurrence of few isolated NFTs could be also detected in brains of non-demented controls, which is in line with the finding that cholinergic neurons express neurofibrillary pathology even during normal aging, but increases significantly in $\mathrm{MCl}$ and worsens in $\mathrm{AD}$ (Mesulam, 2013). A clear presence of $U_{B B}^{+1}$ was also shown in the human AD cases, which is consistent with a previous study (van Leeuwen et al., 2000). The occurrence of early and severe cytopathology of NBM, together with a profound reduction of cholinergic innervation (Whitehouse et al., 1982), may be partly responsible for the existence of a wide range of symptoms AD patients commonly suffer from.

\subsection{Inferior colliculus}

Age-related auditory deficits are quite common in the elderly. AD patients are known to exhibit structural changes (neuronal loss, primary sensory deafferentation) in the central auditory pathways (Sinha et al., 1993). Central auditory dysfunction (CAD) includes individuals hearing well in a quiet environment but having hearing problems in 
environments with a lot of background noise (e.g., competing conversations). Studies demonstrated an increased risk of $A D$ in individuals with $C A D$ and suggest that $C A D$ is an early manifestation of $A D$ that occurs before any sign of cognitive decline. Indeed, people suffering from $\mathrm{MCl}$ showed CAD to a considerable extent (Idrizbegovic et al., 2011). Thus, auditory dysfunction may not only be a valuable tool for diagnosing $A D$ at an early stage, it may also be predictive for the development of $A D$. Moreover there is substantial evidence for neurodegeneration such as senile plaques (Ohm and Braak, 1989) and neurofibrillary tangles (Dugger et al., 2011) in the medial geniculate nucleus ventral part (MGV), the CIC, the primary auditory and auditory association cortices of $A D$ patients.

In the present study, expression of $\mathrm{UBB}^{+1}$ in line 3413 was found in the IC with a density varying from moderate to high. By contrast, in the human brain, $\mathrm{UBB}^{+1}$ immunoreactive substrates were not detected in the IC, which is consistent with the finding that $\mathrm{UBB}^{+1} \mathrm{CO}^{\mathrm{C}}$ localizes with tau pathology, such as NFTs and dystrophic neurites, but not with amyloid depositions (van Leeuwen et al., 1998). In line with other research work (Parvizi et al., 2001), the human immunohistochemical experiments demonstrated that $\mathrm{CIC}$ is severely affected in $A D$, contrary to the external layers of the nucleus (ECIC, DCIC), which expressed very low densities, if any, of immunoreactive substrates (plaques or tangles). Furthermore, the IC expressed selective vulnerability to amyloid pathology, being almost devoid of NFTs. One Braak 5 patient (\#6) showed a high immunoreactivity for (pre)-tangles, however it is related to its presence in NPs.

In conclusion, our experiments demonstrated that high concentrations of AD-related pathology (i.e. MC1, CP13 and $A \beta$ ) affect the inferior colliculi, especially the $\mathrm{CIC}$, in humans. Furthermore, because the Braak 3 case already exhibits severely amyloid depositions, it can be claimed that pathological changes in the nuclei may initiate early in disease's progression. The involvement of the IC, represented by accumulation of plaques, as well as neuronal loss and altered dendrite arborization (Baloyannis et al., 2009), might, therefore, be partly responsible for central auditory symptoms commonly diagnosed in $A D$ patients. 


\subsection{Raphe nuclei}

Depression is a common comorbidity in individuals with $A D$ and may also precede the clinical symptoms of AD by several years (Sierksma et al., 2010). However, it is unclear whether depression is a risk factor (Geerlings et al., 2008) or a prodromal sign (Wilson et al., 2004) for dementia and AD. One of the possible explanations about the pathophysiology of depression is the 'monoaminergic hypothesis' in which a depletion in monoamine levels in the brain i.e., serotonin (5-HT) and noradrenaline (NA) is thought to play a role (Sierksma et al., 2010).

Our human data demonstrated that both $\mathrm{DR}$ and $\mathrm{MnR}$ accumulate $\mathrm{UBB}^{+1}$ positive cells. These results are in line with the finding that aberrant ubiquitin is expressed in comparable brain regions of the $3413 \mathrm{tg}$ mouse. $\mathrm{UBB}^{+1}$ immunoreactive cells showed a low to moderate density of labeling in certain raphe subnuclei of the $3413 \mathrm{tg}$ mice. Intriguingly, the DR receives afferents from the LC. Noradrenergic as well as serotonergic degeneration of the LC is associated with depression (Ressler and Nemeroff, 2000). It was shown in tg mice that LC degeneration causing NA deficiency in AD contributes to early cognitive deficits (Hammerschmidt et al., 2013). As UBB ${ }^{+1}$-ir cells were shown in both the DR and LC of 3413 $\operatorname{tg}$ mice, it is possible that $\mathrm{UBB}^{+1}$ accumulation is associated with depression in $A D$. These results show that there is a correlation with $\mathrm{UBB}^{+1}$ expression in the $\mathrm{DR}$ and the MnR in line 3413. Because accumulation of $\mathrm{UBB}^{+1}$ inhibits proteasomal activity (van Tijn et al., 2007) and because its occurrence in other brain regions is associated with neuropsychological (Fischer et al., 2009) and behavioral (Irmler et al., 2012) phenotypes, it is likely that the presence of $\mathrm{UBB}^{+1}$ positive cells in the raphe nuclei contribute to the abnormal functioning of the 5-HTergic system in $A D$, as well as to the affective symptoms commonly diagnosed in AD patients.

Additionally, the human data showed that nuclei of the rostral raphe complex, namely DR and $\mathrm{MnR}$ are severely affected by NFTs. The caudal raphe complex (RMg, RPa, ROb) was not analyzed, but several studies have already shown that such nuclei are relatively spared in AD (Rub et al., 2000). Consistent with other work (Parvizi et al., 2001), we demonstrated that the DR and MnR are selectively vulnerable to cytoskeletal pathology, being almost devoid of plaques. In addition, it was found that AD-related pathology in the raphe complex 
correlates with disease progression (Rub et al., 2000). The DR manifests lesions at early stages (Braak 1-2), whereas MnR initiates to show pathology later (Braak 3-4). Congruently, the Braak 3 patient analyzed in the present study showed NFTs in DR, but not in MnR. It has been proposed, that neuropathological changes, such as the aggregation of hyperphosphorylated tau, begin in the raphe nuclei, subsequently spreading to the transentorhinal cortex apparently via seeding (Grinberg et al., 2009;Jucker and Walker, 2011). Accordingly, $20 \%$ of Braak 0 cases and $100 \%$ of Braak $\geq 1$ expressed NFTs in the DR (Grinberg et al., 2009). Our findings are consistent with this hypothesis.

In conclusion, the present study expands prior work and shows the global $\mathrm{UBB}^{+1}$ distribution in the brain of the tg mouse line 3413. We selected five regions, namely the OB, Acb, NBM, IC, and raphe nuclei.

The results support the idea that $A D$ is a phenomenon that involves more than forebrain degeneration associated with memory problems. It is shown that the 3413 tg model has some predictive value than previously assumed (Irmler et al., 2012). However, emphasis is needed to the fact that line 3413 is a genetic model which is useful to address loss of protein control but it has also its limitation with respect to $A D$ which is multifactorial. Line 3413 can be used as a read-out possibility for further AD-related research in mice especially when crossed with existing $A D$ lines and other diseases models to show the relevance of other processes such as $A \beta$ plaque formation (van Tijn et al., 2012) and other cytological processes (Schipanski et al., 2014). The present study showed that immunoreactivity for $\mathrm{UBB}^{+1}$ found in the tg mouse model 3413 is not completely mirrored in the AD brain. More specifically, we demonstrated in addition to the brain stem nuclei that areas that present substantial accumulation of tangle bearing neurons, such as NBM and raphei nuclei, present also high densities of $\mathrm{UBB}^{+1}$ positive cells. These data can be used to reveal the impact of proteasomal stress on functioning and on neurodegenerative pathology as shown by GWAS studies in AD (IGAP, 2015) and to uncover new avenues for research not only on AD but also on other multifactorial tauopathies (Fischer et al., 2003) and polyglutamine diseases (such as HD (de Pril et al., 2004) 


\section{List of anatomical abbreviations used in the paper:}

\begin{tabular}{|c|c|}
\hline \multicolumn{2}{|c|}{ Abbreviations } \\
\hline $10 N$ & $\begin{array}{l}\text { dorsal motor nucleus of } \\
\text { vagus }\end{array}$ \\
\hline AAD & $\begin{array}{l}\text { anterior amygdaloid area, } \\
\text { dorsal part }\end{array}$ \\
\hline AAV & $\begin{array}{l}\text { anterior amygdaloid area, } \\
\text { ventral part }\end{array}$ \\
\hline Ac & $\begin{array}{l}\text { nucleus of the anterior } \\
\text { commissure }\end{array}$ \\
\hline Acb & accumbens nucleus \\
\hline $\mathrm{AcbC}$ & accumbens nucleus, core \\
\hline AcbSh & accumbens nucleus, shell \\
\hline $\mathrm{AcCL}$ & $\begin{array}{l}\text { subventricular part of } n \text {. } \\
\text { accumbens }\end{array}$ \\
\hline $\mathrm{AcCM}$ & $\begin{array}{l}\text { centromedial part of } n \text {. } \\
\text { accumbens }\end{array}$ \\
\hline AcM & medial part of $n$. accumbens \\
\hline Aco & $\begin{array}{l}\text { anterior cortical amygdaloid } \\
\text { nucleus }\end{array}$ \\
\hline AcSV & $\begin{array}{l}\text { centrolateral part of } n . \\
\text { accumbens }\end{array}$ \\
\hline Ahi & amygdalohippocampal area \\
\hline AhiAL & $\begin{array}{l}\text { amygdalohippocampal area, } \\
\text { anterolateral part }\end{array}$ \\
\hline AhiPM & $\begin{array}{l}\text { amygdalohippocampal area, } \\
\text { posteromedial part }\end{array}$ \\
\hline AOB & accessory olfactory bulb \\
\hline
\end{tabular}

\begin{tabular}{|c|c|}
\hline \multicolumn{2}{|c|}{ Abbreviations } \\
\hline AOD & $\begin{array}{l}\text { anterior olfactory area, } \\
\text { dorsal part }\end{array}$ \\
\hline AOE & $\begin{array}{l}\text { anterior olfactory area, } \\
\text { external part }\end{array}$ \\
\hline AOL & $\begin{array}{l}\text { anterior olfactory area, } \\
\text { lateral part }\end{array}$ \\
\hline AOM & $\begin{array}{l}\text { anterior olfactory area, } \\
\text { medial part }\end{array}$ \\
\hline AON & anterior olfactory nucleus \\
\hline AOP & $\begin{array}{l}\text { anterior olfactory area, } \\
\text { posterior part }\end{array}$ \\
\hline AOV & $\begin{array}{l}\text { anterior olfactory area, } \\
\text { ventral part }\end{array}$ \\
\hline AP & area postrema \\
\hline Apir & $\begin{array}{l}\text { amygdalopiriform transition } \\
\text { area }\end{array}$ \\
\hline $\mathrm{Aq}$ & cerebral aqueduct \\
\hline Astr & $\begin{array}{l}\text { amygdalostriatal transition } \\
\text { area }\end{array}$ \\
\hline BAOT & $\begin{array}{l}\text { bed nucleus of the } \\
\text { accessory olfactory tract }\end{array}$ \\
\hline $\mathrm{BIC}$ & $\begin{array}{l}\text { nucleus of the brachium of } \\
\text { the inferior colliculus }\end{array}$ \\
\hline BLA & $\begin{array}{l}\text { basolateral amygdaloid } \\
\text { nucleus, anterior part }\end{array}$ \\
\hline BLP & $\begin{array}{l}\text { basolateral amygdaloid } \\
\text { nucleus, posterior part }\end{array}$ \\
\hline
\end{tabular}




\begin{tabular}{|c|c|}
\hline \multicolumn{2}{|c|}{ Abbreviations } \\
\hline BLV & $\begin{array}{l}\text { basolateral amygdaloid } \\
\text { nucleus, ventral part }\end{array}$ \\
\hline BMA & $\begin{array}{l}\text { basomedial amygdaloid } \\
\text { nucleus, anterior part }\end{array}$ \\
\hline BMP & $\begin{array}{l}\text { basomedial amygdaloid } \\
\text { nucleus, posterior part }\end{array}$ \\
\hline BST & $\begin{array}{l}\text { bed nucleus of the stria } \\
\text { terminalis }\end{array}$ \\
\hline CA1 & $\begin{array}{l}\text { cornu ammonis } 1 \text { (CA1) of } \\
\text { hippocampus }\end{array}$ \\
\hline CA2 & $\begin{array}{l}\text { cornu ammonis } 2 \text { (CA2) of } \\
\text { hippocampus }\end{array}$ \\
\hline CA3 & $\begin{array}{l}\text { cornu ammonis } 3 \text { (CA3) of } \\
\text { hippocampus }\end{array}$ \\
\hline $\mathrm{Cb}$ & Cerebellum \\
\hline $\mathrm{Cd}$ & caudate nucleus \\
\hline $\mathrm{CeC}$ & $\begin{array}{l}\text { central amygdaloid nucleus } \\
\text { capsular part }\end{array}$ \\
\hline CeL & $\begin{array}{l}\text { central amygdaloid nucleus, } \\
\text { lateral division }\end{array}$ \\
\hline CeM & $\begin{array}{l}\text { central amygdaloid nucleus, } \\
\text { medial division }\end{array}$ \\
\hline CeMAD & $\begin{array}{l}\text { central amygdaloid nucleus, } \\
\text { medial division, } \\
\text { anterodorsal part }\end{array}$ \\
\hline CeMAV & $\begin{array}{l}\text { central amygdaloid nucleus, } \\
\text { medial division, } \\
\text { anteroventral part }\end{array}$ \\
\hline CeMPV & $\begin{array}{l}\text { central amygdaloid nucleus, } \\
\text { medial posteroventral part }\end{array}$ \\
\hline
\end{tabular}

\begin{tabular}{|c|c|}
\hline \multicolumn{2}{|c|}{ Abbreviations } \\
\hline CEnt & $\begin{array}{l}\text { caudomedial entothinal } \\
\text { cortex }\end{array}$ \\
\hline Cg1 & cingulate cortex, area 1 \\
\hline $\mathrm{Cg} 2$ & cingulate cortex, area 2 \\
\hline $\mathrm{CIC}$ & $\begin{array}{l}\text { central nucleus of the } \\
\text { inferior colliculus }\end{array}$ \\
\hline Cli & $\begin{array}{l}\text { caudal linear nucleus of the } \\
\text { raphe }\end{array}$ \\
\hline $\mathrm{CPu}$ & caudate putamen (striatum) \\
\hline $\mathrm{Cx}$ & cerebral cortex \\
\hline CXA & $\begin{array}{l}\text { cortex-amygdala transition } \\
\text { zone }\end{array}$ \\
\hline DC & dorsal cochlear nucleus \\
\hline $\mathrm{DCIC}$ & $\begin{array}{l}\text { dorsal cortex of the } \\
\text { inferior colliculus }\end{array}$ \\
\hline Den & dorsal endopiriform nucleus \\
\hline DP & dorsal peduncular cortex \\
\hline DR & dorsal raphe nucleus \\
\hline DRC & $\begin{array}{l}\text { dorsal raphe nucleus, } \\
\text { caudal part }\end{array}$ \\
\hline DRD & $\begin{array}{l}\text { dorsal raphe nucleus, dorsal } \\
\text { part }\end{array}$ \\
\hline DRI & $\begin{array}{l}\text { dorsal raphe nucleus, } \\
\text { interfascicular part }\end{array}$ \\
\hline DRV & $\begin{array}{l}\text { dorsal raphe nucleus, } \\
\text { ventral part }\end{array}$ \\
\hline DRVL & $\begin{array}{l}\text { dorsal raphe nucleus, } \\
\text { ventrolateral part }\end{array}$ \\
\hline
\end{tabular}




\begin{tabular}{|c|c|}
\hline \multicolumn{2}{|c|}{ Abbreviations } \\
\hline DTT & dorsal tenia tecta \\
\hline Ec & external capsule \\
\hline $\mathrm{ECIC}$ & $\begin{array}{l}\text { external cortex of the } \\
\text { inferior colliculus }\end{array}$ \\
\hline Ect & ectorhinal cortex \\
\hline E/OV & $\begin{array}{l}\text { ependymal and subendyma } \\
\text { layer/olfactory ventricle }\end{array}$ \\
\hline Epl & $\begin{array}{l}\text { external plexiform layer of } \\
\text { the olfactory bulb }\end{array}$ \\
\hline EplA & $\begin{array}{l}\text { external plexiform layer of } \\
\text { the accessory olfactory bulb }\end{array}$ \\
\hline FC & fasciola cinereum \\
\hline $\mathrm{FCd}$ & $\begin{array}{l}\text { nucleus accumbens, } \\
\text { caudate fundus }\end{array}$ \\
\hline Fpu & $\begin{array}{l}\text { nucleus accumbens, } \\
\text { putaminal fundus }\end{array}$ \\
\hline FrA & frontal association cortex \\
\hline $\mathrm{Gi}$ & $\begin{array}{l}\text { gigantocellular reticular } \\
\text { nucleus }\end{array}$ \\
\hline Gl & $\begin{array}{l}\text { glomerular layer of the } \\
\text { olfactory bulb }\end{array}$ \\
\hline GIA & $\begin{array}{l}\text { glomerular layer of the } \\
\text { accessory olfactory bulb }\end{array}$ \\
\hline GP & globus pallidus \\
\hline Gpe & $\begin{array}{l}\text { globus pallidus, external } \\
\text { segment }\end{array}$ \\
\hline Gpi & $\begin{array}{l}\text { globus pallidus, internal } \\
\text { segment }\end{array}$ \\
\hline
\end{tabular}

\begin{tabular}{|l|l|}
\hline \multicolumn{2}{|l|}{ Abbreviations } \\
\hline GrA & $\begin{array}{l}\text { granule cell layer of the } \\
\text { accessory olfactory bulb }\end{array}$ \\
\hline GrC & $\begin{array}{l}\text { granular layer of the } \\
\text { cochlear nuclei }\end{array}$ \\
\hline GrDG & $\begin{array}{l}\text { granular layer of the dentate } \\
\text { gyrus }\end{array}$ \\
\hline GrO & $\begin{array}{l}\text { granular cell layer of the } \\
\text { olfactory bulb }\end{array}$ \\
\hline Hip & Hippocampus \\
\hline HTh & Hypothalamus \\
\hline I & $\begin{array}{l}\text { intercalated nuclei of the } \\
\text { amygdala }\end{array}$ \\
\hline IC & internal capsule \\
\hline IC & inferior colliculus \\
\hline Icj & islands of Calleja \\
\hline IcjM & $\begin{array}{l}\text { islands of Calleja, major } \\
\text { island }\end{array}$ \\
\hline Ig & indusium griseum \\
\hline IL & infralimbic cortex \\
\hline IM & $\begin{array}{l}\text { intercalated amygdaloid } \\
\text { nucleus, main part }\end{array}$ \\
\hline Ipl & $\begin{array}{l}\text { internal plexiform layer of } \\
\text { the olfactory bulb }\end{array}$ \\
\hline L1 & cortical layer 1 \\
\hline L2 & cortical layer 2 \\
\hline L3 & cortical layer 3 \\
\hline cortical layer 4
\end{tabular}




\begin{tabular}{|c|c|}
\hline \multicolumn{2}{|c|}{ Abbreviations } \\
\hline L5a & cortical layer $5 a$ \\
\hline L5b & cortical layer 5b \\
\hline L6 & cortical layer 6 \\
\hline La & lateral amygdaloid nucleus \\
\hline LAcbSh & lateral accumbens shell \\
\hline LaDL & $\begin{array}{l}\text { lateral amygdaloid nucleus, } \\
\text { dorsolateral part }\end{array}$ \\
\hline LaVL & $\begin{array}{l}\text { lateral amygdaloid nucleus, } \\
\text { ventromedial part }\end{array}$ \\
\hline LaVM & $\begin{array}{l}\text { lateral amygdaloid nucleus, } \\
\text { ventromedial part }\end{array}$ \\
\hline LC & locus coeruleus \\
\hline LGP & lateral globus pallidus \\
\hline Iml & lateral medullary lamina \\
\hline LMol & $\begin{array}{l}\text { lacunosum moleculare layer } \\
\text { of the hippocampus }\end{array}$ \\
\hline LO & lateral orbital cortex \\
\hline LOT & $\begin{array}{l}\text { nucleus of the lateral } \\
\text { olfactory tract }\end{array}$ \\
\hline LPB & lateral parabrachial nucleus \\
\hline LPBC & $\begin{array}{l}\text { lateral parabrachial nucleus, } \\
\text { central part }\end{array}$ \\
\hline LPBD & $\begin{array}{l}\text { lateral parabrachial nucleus, } \\
\text { dorsal part }\end{array}$ \\
\hline LPBE & $\begin{array}{l}\text { lateral parabrachial nucleus, } \\
\text { external part }\end{array}$ \\
\hline LPBI & $\begin{array}{l}\text { lateral parabrachial nucleus, } \\
\text { internal part }\end{array}$ \\
\hline
\end{tabular}

\begin{tabular}{|c|c|}
\hline \multicolumn{2}{|c|}{ Abbreviations } \\
\hline LPBV & $\begin{array}{l}\text { lateral parabrachial nucleus, } \\
\text { ventral part }\end{array}$ \\
\hline LS & lateral septal nucleus \\
\hline LSO & lateral superior olive \\
\hline LSS & lateral stripe of the striatum \\
\hline LV & lateral ventricle \\
\hline M2 & secondary motor cortex \\
\hline $\mathrm{MeA}$ & $\begin{array}{l}\text { medial amygdaloid nucleus, } \\
\text { anterior part }\end{array}$ \\
\hline MeAD & $\begin{array}{l}\text { medial amygdaloid nucleus, } \\
\text { anterior dorsal part }\end{array}$ \\
\hline MeAV & $\begin{array}{l}\text { medial amygdaloid nucleus, } \\
\text { anteroventral part }\end{array}$ \\
\hline MePD & $\begin{array}{l}\text { medial amygdaloid nucleus, } \\
\text { posterodorsal part }\end{array}$ \\
\hline MePV & $\begin{array}{l}\text { medial amygdaloid nucleus, } \\
\text { posteroventral part }\end{array}$ \\
\hline MG & medial geniculate nucleus \\
\hline MGD & $\begin{array}{l}\text { medial geniculate nucleus, } \\
\text { dorsal part }\end{array}$ \\
\hline MGM & $\begin{array}{l}\text { medial geniculate nucleus, } \\
\text { medial part }\end{array}$ \\
\hline MGP & medial globus pallidus \\
\hline MGV & $\begin{array}{l}\text { medial geniculate nucleus, } \\
\text { ventral part }\end{array}$ \\
\hline Mi & $\begin{array}{l}\text { mitral cell layer of the } \\
\text { olfactory bulb }\end{array}$ \\
\hline $\mathrm{MiA}$ & $\begin{array}{l}\text { mitral cell layer of the } \\
\text { accessory olfactory bulb }\end{array}$ \\
\hline
\end{tabular}




\begin{tabular}{|c|c|}
\hline \multicolumn{2}{|c|}{ Abbreviations } \\
\hline Mlf & $\begin{array}{l}\text { medial longitudinal } \\
\text { fasciculus }\end{array}$ \\
\hline $\mathrm{Mml}$ & medial medullary lamina \\
\hline $\mathrm{mMnR}$ & $\begin{array}{l}\text { median raphe nucleus, } \\
\text { medial part }\end{array}$ \\
\hline MnR & median raphe nucleus \\
\hline Mol & $\begin{array}{l}\text { molecular layer of the } \\
\text { dendate gyrus }\end{array}$ \\
\hline MPB & medial parabrachial nucleus \\
\hline MPBe & $\begin{array}{l}\text { medial parabrachial nucleus } \\
\text { external part }\end{array}$ \\
\hline MZMG & $\begin{array}{l}\text { marginal zone of the } \\
\text { medial geniculate }\end{array}$ \\
\hline NBM & nucleus basalis of Meynert \\
\hline NCS & nucleus centralis superior \\
\hline OB & olfactory bulb \\
\hline OlfA & olfactory area \\
\hline PAG & periaqueductal gray \\
\hline PaS & parasubiculum \\
\hline PBW & $\begin{array}{l}\text { parabrachial nucleus waist } \\
\text { part }\end{array}$ \\
\hline Pir & piriform cortex \\
\hline PLCo & $\begin{array}{l}\text { posterolateral cortical } \\
\text { amygdaloid nucleus (C2) }\end{array}$ \\
\hline PMCo & $\begin{array}{l}\text { posteromedial cortical } \\
\text { amygdaloid nucleus (C3) }\end{array}$ \\
\hline PMnR & paramedian raphe nucleus \\
\hline
\end{tabular}

\begin{tabular}{|l|l|}
\hline Abbreviations \\
\hline PnC & $\begin{array}{l}\text { pontine reticular nucleus, } \\
\text { caudal part }\end{array}$ \\
\hline PnO & $\begin{array}{l}\text { pontine reticular nucleus, } \\
\text { oral part }\end{array}$ \\
\hline PnR & pontine raphe nucleus \\
\hline PoDG & $\begin{array}{l}\text { polymorph layer of the } \\
\text { dentate gyrus }\end{array}$ \\
\hline PrL & prelimbic cortex \\
\hline PrS & presubiculum \\
\hline PSol & parasolitary nucleus \\
\hline Pu & putamen \\
\hline PuV & $\begin{array}{l}\text { retrosplenial granular } \\
\text { cortex, b part }\end{array}$ \\
\hline Py & $\begin{array}{l}\text { petras area of putamen } \\
\text { portex, a part }\end{array}$ \\
\hline hippocampus \\
\hline Rad
\end{tabular}




\begin{tabular}{|c|c|}
\hline \multicolumn{2}{|c|}{ Abbreviations } \\
\hline RSGc & $\begin{array}{l}\text { retrosplenial granular } \\
\text { cortex, c part }\end{array}$ \\
\hline S & septal area \\
\hline $\mathrm{Sb}$ & Subiculum \\
\hline $\mathrm{SC}$ & superior colliculus \\
\hline Shi & septohippocampal nucleus \\
\hline SLEA & $\begin{array}{l}\text { sublenticular extended } \\
\text { amygdala }\end{array}$ \\
\hline SLEAC & $\begin{array}{l}\text { sublenticular extended } \\
\text { amygdala, central part }\end{array}$ \\
\hline SLEAM & $\begin{array}{l}\text { sublenticular extended } \\
\text { amygdala, medial part }\end{array}$ \\
\hline Slu & $\begin{array}{l}\text { stratum lucidum, } \\
\text { hippocampus }\end{array}$ \\
\hline Sol & nucleus of the solitary tract \\
\hline Sol & solitary tract \\
\hline SolC & $\begin{array}{l}\text { nucleus of the solitary tract, } \\
\text { commissural part }\end{array}$ \\
\hline SolCe & $\begin{array}{l}\text { nucleus of the solitary tract, } \\
\text { central part }\end{array}$ \\
\hline SolDL & $\begin{array}{l}\text { nucleus of the solitary tract, } \\
\text { dorsolateral part }\end{array}$ \\
\hline SolDM & $\begin{array}{l}\text { nucleus of the solitary tract, } \\
\text { dorsomedial part }\end{array}$ \\
\hline SolG & $\begin{array}{l}\text { nucleus of the solitary tract, } \\
\text { gelatinous part }\end{array}$ \\
\hline Soll & $\begin{array}{l}\text { nucleus of the solitary tract, } \\
\text { interstitial part }\end{array}$ \\
\hline
\end{tabular}

\begin{tabular}{|l|l|}
\hline \multicolumn{2}{|l|}{ Abbreviations } \\
\hline SolIM & $\begin{array}{l}\text { nucleus of the solitary tract, } \\
\text { intermediate part }\end{array}$ \\
\hline SolM & $\begin{array}{l}\text { nucleus of the solitary tract, } \\
\text { medial part }\end{array}$ \\
\hline SolV & $\begin{array}{l}\text { nucleus of the solitary tract, } \\
\text { ventral part }\end{array}$ \\
\hline SolVL & $\begin{array}{l}\text { nucleus of the solitary tract, } \\
\text { ventrolateral part }\end{array}$ \\
\hline STh & subthalamic nucleus \\
\hline Th & Thalamus \\
\hline Tu & olfactory tubercle \\
\hline Tz & $\begin{array}{l}\text { nucleus of the trapezoid } \\
\text { body }\end{array}$ \\
\hline V1 & primary visual cortex \\
\hline V2ML & $\begin{array}{l}\text { secondary visual cortex, } \\
\text { mediolateral area }\end{array}$ \\
\hline V2MM & $\begin{array}{l}\text { secondary visual cortex, } \\
\text { mediomedial area }\end{array}$ \\
\hline VC & ventral cochlear nucleus \\
\hline VCA & $\begin{array}{l}\text { ventral cochlear nucleus, } \\
\text { anterior part }\end{array}$ \\
\hline VCP & $\begin{array}{l}\text { ventral cochlear nucleus, } \\
\text { posterior part }\end{array}$ \\
\hline VEn & ventral endopiriform nucleus \\
\hline ventral orbital cortex \\
ventral tenia tecta \\
\hline VTT
\end{tabular}




\section{References}

Attems, J., Walker, L., and Jellinger, K.A. (2014). Olfactory bulb involvement in neurodegenerative diseases. Acta Neuropathol 127, 459-475.

Baloyannis, S.J., Mauroudis, I., Manolides, S.L., and Manolides, L.S. (2009). Synaptic alterations in the medial geniculate bodies and the inferior colliculi in Alzheimer's disease: a Golgi and electron microscope study. Acta oto-laryngologica 129, 416-418.

Barnes, D.E., and Yaffe, K. (2011). The projected effect of risk factor reduction on Alzheimer's disease prevalence. Lancet Neurology 10, 819-828.

Braak, H., Del Tredici, K., Rub, U., De Vos, R.A., Jansen Steur, E.N., and Braak, E. (2003). Staging of brain pathology related to sporadic Parkinson's disease. Neurobiology of aging 24, 197211.

Braak, H., Thal, D.R., Ghebremedhin, E., and Del Tredici, K. (2011). Stages of the pathologic process in Alzheimer disease: age categories from 1 to 100 years. Journal of neuropathology and experimental neurology 70, 960-969.

Brilliant, M.J., Elble, R.J., Ghobrial, M., and Struble, R.G. (1997). The distribution of amyloid beta protein deposition in the corpus striatum of patients with Alzheimer's disease. Neuropathol Appl Neurobiol 23, 322-325.

Burns, J.M., Mayo, M.S., Anderson, H.S., Smith, H.J., and Donnelly, J.E. (2008). Cardiorespiratory fitness in early-stage Alzheimer disease. Alzheimer Dis Assoc Disord 22, 39-46.

Cereda, E., Cilia, R., Klersy, C., Canesi, M., Zecchinelli, A.L., Mariani, C.B., Tesei, S., Sacilotto, G., Meucci, N., Zini, M., Isaias, I.U., Cassani, E., Goldwurm, S., Barichella, M., and Pezzoli, G. (2014). Swallowing disturbances in Parkinson's disease: A multivariate analysis of contributing factors. Parkinsonism Relat Disord 20, 1382-1387.

Corder, E.H., Saunders, A.M., Strittmatter, W.J., Schmechel, D.E., Gaskell, P.C., Small, G.W., Roses, A.D., Haines, J.L., and Pericak-Vance, M.A. (1993). Gene dose of apolipoprotein E type 4 allele and the risk of Alzheimer's disease in late onset families. Science 261, 921923.

De Jong, L.W., Ferrarini, L., Van Der Grond, J., Milles, J.R., Reiber, J.H., Westendorp, R.G., Bollen, E.L., Middelkoop, H.A., and Van Buchem, M.A. (2011). Shape abnormalities of the striatum in Alzheimer's disease. Journal of Alzheimer's Disease 23, 49-59.

De Pril, R., Fischer, D.F., Maat-Schieman, M.L., Hobo, B., De Vos, R.A., Brunt, E.R., Hol, E.M., Roos, R.A., and Van Leeuwen, F.W. (2004). Accumulation of aberrant ubiquitin induces aggregate formation and cell death in polyglutamine diseases. Human molecular genetics 13, 18031813.

Del Tredici, K., Rub, U., De Vos, R.A., Bohl, J.R., and Braak, H. (2002). Where does parkinson disease pathology begin in the brain? J Neuropathol Exp Neurol 61, 413-426.

Dennissen, F.J., Kholod, N., and Van Leeuwen, F.W. (2012). The ubiquitin proteasome system in neurodegenerative diseases: culprit, accomplice or victim? Progress in neurobiology 96, 190-207.

Djordjevic, J., Jones-Gotman, M., De Sousa, K., and Chertkow, H. (2008). Olfaction in patients with mild cognitive impairment and Alzheimer's disease. Neurobiology of Aging 29, 693-706. 
Dugger, B.N., Tu, M., Murray, M.E., and Dickson, D.W. (2011). Disease specificity and pathologic progression of tau pathology in brainstem nuclei of Alzheimer's disease and progressive supranuclear palsy. Neuroscience letters 491, 122-126.

Duyckaerts, C., Delatour, B., and Potier, M.C. (2009). Classification and basic pathology of Alzheimer disease. Acta Neuropathol 118, 5-36.

Fischer, D.F., De Vos, R.A., Van Dijk, R., De Vrij, F.M., Proper, E.A., Sonnemans, M.A., Verhage, M.C., Sluijs, J.A., Hobo, B., Zouambia, M., Steur, E.N., Kamphorst, W., Hol, E.M., and Van Leeuwen, F.W. (2003). Disease-specific accumulation of mutant ubiquitin as a marker for proteasomal dysfunction in the brain. FASEB journal : official publication of the Federation of American Societies for Experimental Biology 17, 2014-2024.

Fischer, D.F., Van Dijk, R., Van Tijn, P., Hobo, B., Verhage, M.C., Van Der Schors, R.C., Li, K.W., Van Minnen, J., Hol, E.M., and Van Leeuwen, F.W. (2009). Long-term proteasome dysfunction in the mouse brain by expression of aberrant ubiquitin. Neurobiology of Aging 30, 847-863.

Franklin, K.B.J., and Paxinos, J. (2007). The mouse brain in stereotaxic coordinates. . Amsterdam: Elsevier.

Geerlings, M.I., Den Heijer, T., Koudstaal, P.J., Hofman, A., and Breteler, M.M. (2008). History of depression, depressive symptoms, and medial temporal lobe atrophy and the risk of Alzheimer disease. Neurology 70, 1258-1264.

Grinberg, L.T., Rub, U., Ferretti, R.E., Nitrini, R., Farfel, J.M., Polichiso, L., Gierga, K., Jacob-Filho, W., and Heinsen, H. (2009). The dorsal raphe nucleus shows phospho-tau neurofibrillary changes before the transentorhinal region in Alzheimer's disease. A precocious onset? Neuropathology and applied neurobiology 35, 406-416.

Guerin, D., Sacquet, J., Mandairon, N., Jourdan, F., and Didier, A. (2009). Early locus coeruleus degeneration and olfactory dysfunctions in Tg2576 mice. Neurobiology of aging 30, 272283.

Haines, D.E. (2011). Neuroanatomy: An Atlas of Structures, Sections and Systems. Wolters Kluwer/Lippincott Williams \& Wilkins Health.

Hammerschmidt, T., Kummer, M.P., Terwel, D., Martinez, A., Gorji, A., Pape, H.C., Rommelfanger, K.S., Schroeder, J.P., Stoll, M., Schultze, J., Weinshenker, D., and Heneka, M.T. (2013). Selective loss of noradrenaline exacerbates early cognitive dysfunction and synaptic deficits in APP/PS1 mice. Biological psychiatry 73, 454-463.

Heneka, M.T., Ramanathan, M., Jacobs, A.H., Dumitrescu-Ozimek, L., Bilkei-Gorzo, A., Debeir, T., Sastre, M., Galldiks, N., Zimmer, A., Hoehn, M., Heiss, W.D., Klockgether, T., and Staufenbiel, M. (2006). Locus ceruleus degeneration promotes Alzheimer pathogenesis in amyloid precursor protein 23 transgenic mice. The Journal of neuroscience : the official journal of the Society for Neuroscience 26, 1343-1354.

Holton, P., Ryten, M., and Nalls, M. (2013). Initial assessment of the pathogenic mechanisms of the recently identified Alzheimer risk Loci. Annals of human genetics 77, 85-105.

Idrizbegovic, E., Hederstierna, C., Dahlquist, M., Kampfe Nordstrom, C., Jelic, V., and Rosenhall, U. (2011). Central auditory function in early Alzheimer's disease and in mild cognitive impairment. Age and ageing 40, 249-254. 
Igap (2015). International Genomics of Alzheimer's Disease Consortium. Convergent genetic and expression data implicate immunity in Alzheimer's disease. Alzheimer's \& Dementia 11, 658-671.

Irmler, M., Gentier, R.J., Dennissen, F.J., Schulz, H., Bolle, I., Holter, S.M., Kallnik, M., Cheng, J.J., Klingenspor, M., Rozman, J., Ehrhardt, N., Hermes, D.J., Gailus-Durner, V., Fuchs, H., Hrabe De Angelis, M., Meyer, H.E., Hopkins, D.A., Van Leeuwen, F.W., and Beckers, J. (2012). Long-term proteasomal inhibition in transgenic mice by UBB(+1) expression results in dysfunction of central respiration control reminiscent of brainstem neuropathology in Alzheimer patients. Acta neuropathologica 124, 187-197.

Jankowsky, J.L., Fadale, D.J., Anderson, J., Xu, G.M., Gonzales, V., Jenkins, N.A., Copeland, N.G., Lee, M.K., Younkin, L.H., Wagner, S.L., Younkin, S.G., and Borchelt, D.R. (2004). Mutant presenilins specifically elevate the levels of the 42 residue beta-amyloid peptide in vivo: evidence for augmentation of a 42-specific gamma secretase. Human molecular genetics 13, 159-170.

Jellinger, K.A. (2014). The cholinergic basal forebrain in Lewy body dementia and Alzheimer's disease. J Neurol.

Jonsson, T., Stefansson, H., Steinberg, S., Jonsdottir, I., Jonsson, P.V., Snaedal, J., Bjornsson, S., Huttenlocher, J., Levey, A.I., Lah, J.J., Rujescu, D., Hampel, H., Giegling, I., Andreassen, O.A., Engedal, K., Ulstein, I., Djurovic, S., Ibrahim-Verbaas, C., Hofman, A., Ikram, M.A., Van Duijn, C.M., Thorsteinsdottir, U., Kong, A., and Stefansson, K. (2013). Variant of TREM2 associated with the risk of Alzheimer's disease. The New England journal of medicine 368, 107-116.

Jucker, M., and Walker, L.C. (2011). Pathogenic protein seeding in Alzheimer disease and other neurodegenerative disorders. Ann Neurol 70, 532-540.

Krutauz, D., Reis, N., Nakasone, M.A., Siman, P., Zhang, D., Kirkpatrick, D.S., Gygi, S.P., Brik, A., Fushman, D., and Glickman, M.H. (2014). Extended ubiquitin species are protein-based DUB inhibitors. Nat Chem Biol 10, 664-670.

Mai, J.K., and Paxinos, G. (2012). The Human Nervous system. Amsterdam: Elsevier Science

Mai, J.K., Paxinos, G., and Voss, T. (2008). Atlas of the human brain. Academic Press.

Manavalan, A., Mishra, M., Feng, L., Sze, S.K., Akatsu, H., and Heese, K. (2013). Brain site-specific proteome changes in aging-related dementia. Experimental \& Molecular Medicine 45, e39.

Mayford, M., Bach, M.E., Huang, Y.Y., Wang, L., Hawkins, R.D., and Kandel, E.R. (1996a). Control of memory formation through regulated expression of a CaMKII transgene. Science 274, 1678-1683.

Mayford, M., Bach, M.E., and Kandel, E. (1996b). CaMKII function in the nervous system explored from a genetic perspective. Cold Spring Harbor symposia on quantitative biology 61, 219224.

Mesulam, M., Shaw, P., Mash, D., and Weintraub, S. (2004). Cholinergic nucleus basalis tauopathy emerges early in the aging-MCl-AD continuum. Ann Neurol 55, 815-828.

Mesulam, M.M. (2013). Cholinergic circuitry of the human nucleus basalis and its fate in Alzheimer's disease. J Comp Neurol 521, 4124-4144. 
Mitchell, R.A., Herrmann, N., and Lanctot, K.L. (2011). The role of dopamine in symptoms and treatment of apathy in Alzheimer's disease. CNS Neuroscience \& Therapeutics 17, 411427.

Mobley, A.S., Rodriguez-Gil, D.J., Imamura, F., and Greer, C.A. (2014). Aging in the olfactory system. Trends Neurosci 37, 77-84.

Murray, A.M., Weihmueller, F.B., Marshall, J.F., Hurtig, H.I., Gottleib, G.L., and Joyce, J.N. (1995). Damage to dopamine systems differs between Parkinson's disease and Alzheimer's disease with parkinsonism. Annals of neurology 37, 300-312.

Ohm, T.G., and Braak, H. (1987). Olfactory bulb changes in Alzheimer's disease. Acta Neuropathol 73, 365-369.

Ohm, T.G., and Braak, H. (1989). Auditory brainstem nuclei in Alzheimer's disease. Neuroscience letters 96, 60-63.

Parvizi, J., Van Hoesen, G.W., and Damasio, A. (2001). The selective vulnerability of brainstem nuclei to Alzheimer's disease. Annals of neurology 49, 53-66.

Petry, F.R., Pelletier, J., Bretteville, A., Morin, F., Calon, F., Hebert, S.S., Whittington, R.A., and Planel, E. (2014). Specificity of anti-tau antibodies when analyzing mice models of Alzheimer's disease: problems and solutions. PLoS One 9, e94251.

Ressler, K.J., and Nemeroff, C.B. (2000). Role of serotonergic and noradrenergic systems in the pathophysiology of depression and anxiety disorders. Depression and anxiety 12 Suppl 1, 2-19.

Rey, N.L., Jardanhazi-Kurutz, D., Terwel, D., Kummer, M.P., Jourdan, F., Didier, A., and Heneka, M.T. (2012). Locus coeruleus degeneration exacerbates olfactory deficits in APP/PS1 transgenic mice. Neurobiology of aging 33, 426 e421-411.

Rub, U., Del Tredici, K., Schultz, C., Thal, D.R., Braak, E., and Braak, H. (2000). The evolution of Alzheimer's disease-related cytoskeletal pathology in the human raphe nuclei. Neuropathology and applied neurobiology 26, 553-567.

Schipanski, A., Oberhauser, F., Neumann, M., Lange, S., Szalay, B., Krasemann, S., Van Leeuwen, F.W., Galliciotti, G., and Glatzel, M. (2014). The lectin OS-9 delivers mutant neuroserpin to endoplasmic reticulum associated degradation in familial encephalopathy with neuroserpin inclusion bodies. Neurobiol Aging 35, 2394-2403.

Selkoe, D.J. (2001). Alzheimer's disease: genes, proteins, and therapy. Physiological reviews 81, 741-766.

Sierksma, A.S., Van Den Hove, D.L., Steinbusch, H.W., and Prickaerts, J. (2010). Major depression, cognitive dysfunction and Alzheimer's disease: is there a link? European Journal of Pharmacology 626, 72-82.

Sinha, U.K., Hollen, K.M., Rodriguez, R., and Miller, C.A. (1993). Auditory system degeneration in Alzheimer's disease. Neurology 43, 779-785.

Suenaga, T., Hirano, A., Llena, J.F., Yen, S.H., and Dickson, D.W. (1990). Modified Bielschowsky stain and immunohistochemical studies on striatal plaques in Alzheimer's disease. Acta neuropathologica 80, 280-286.

Van Leeuwen, F.W., De Kleijn, D.P., Van Den Hurk, H.H., Neubauer, A., Sonnemans, M.A., Sluijs, J.A., Koycu, S., Ramdjielal, R.D., Salehi, A., Martens, G.J., Grosveld, F.G., Peter, J., 
Burbach, H., and Hol, E.M. (1998). Frameshift mutants of beta amyloid precursor protein and ubiquitin-B in Alzheimer's and Down patients. Science 279, 242-247.

Van Leeuwen, F.W., Fischer, D.F., Kamel, D., Sluijs, J.A., Sonnemans, M.A., Benne, R., Swaab, D.F., Salehi, A., and Hol, E.M. (2000). Molecular misreading: a new type of transcript mutation expressed during aging. Neurobiology of aging 21, 879-891.

Van Leeuwen, F.W., Van Tijn, P., Sonnemans, M.A., Hobo, B., Mann, D.M., Van Broeckhoven, C., Kumar-Singh, S., Cras, P., Leuba, G., Savioz, A., Maat-Schieman, M.L., Yamaguchi, H., Kros, J.M., Kamphorst, W., Hol, E.M., De Vos, R.A., and Fischer, D.F. (2006). Frameshift proteins in autosomal dominant forms of Alzheimer disease and other tauopathies. Neurology 66, S86-92.

Van Tijn, P., De Vrij, F.M., Schuurman, K.G., Dantuma, N.P., Fischer, D.F., Van Leeuwen, F.W., and Hol, E.M. (2007). Dose-dependent inhibition of proteasome activity by a mutant ubiquitin associated with neurodegenerative disease. Journal of Cell Science 120, 1615-1623.

Van Tijn, P., Dennissen, F.J., Gentier, R.J., Hobo, B., Hermes, D., Steinbusch, H.W., Van Leeuwen, F.W., and Fischer, D.F. (2012). Mutant ubiquitin decreases amyloid beta plaque formation in a transgenic mouse model of Alzheimer's disease. Neurochemistry international 61, 739748.

Van Tijn, P., Hobo, B., Verhage, M.C., Oitzl, M.S., Van Leeuwen, F.W., and Fischer, D.F. (2011). Alzheimer-associated mutant ubiquitin impairs spatial reference memory. Physiology \& behavior 102, 193-200.

Vidoni, E.D., Honea, R.A., Billinger, S.A., Swerdlow, R.H., and Burns, J.M. (2012). Cardiorespiratory fitness is associated with atrophy in Alzheimer's and aging over 2 years. Neurobiol Aging 33, 1624-1632.

Whitehouse, P.J., Price, D.L., Struble, R.G., Clark, A.W., Coyle, J.T., and Delon, M.R. (1982). Alzheimer's disease and senile dementia: loss of neurons in the basal forebrain. Science 215, 1237-1239.

Wilson, R.S., Mendes De Leon, C.F., Bennett, D.A., Bienias, J.L., and Evans, D.A. (2004). Depressive symptoms and cognitive decline in a community population of older persons. Journal of Neurology, Neurosurgery, and Psychiatry 75, 126-129. 



\section{CHAPTER 3}

Long-term proteasomal inhibition in transgenic mice by $\mathrm{UBB}^{+1}$ expression results in dysfunction of central respiration control reminiscent of brainstem neuropathology in Alzheimer patients

R.J. Gentier*, M. Irmler*, F.J.A. Dennissen, H. Schulz, I. Bolle, S.M. Hölter, M. Kallnik, J. Cheng, M. Klingenspor, J. Rozman, N. Ehrhardt, D.J.H.P. Hermes, V. Gailus-Durner, H. Fuchs, M. Hrabě de Angelis, H.E. Meyer, D.A. Hopkins, F.W. Van Leeuwen ${ }^{* \star}$, J. Beckers ${ }^{* *}$, Acta Neuropathologica.2012;124:187-197

* contributed equally; ${ }^{* *}$ combined last authors

Supplementary material available at:

http://link.springer.com/article/10.1007\%2Fs00401-012-1003-7 


\section{Abstract}

Aging and neurodegeneration are often accompanied by a functionally impaired ubiquitinproteasome system (UPS). In tauopathies and polyglutamine diseases a mutant form of Ubiquitin $\mathrm{B}, \mathrm{UBB}^{+1}$, accumulates in disease-specific aggregates. $\mathrm{UBB}^{+1} \mathrm{mRNA}$ is generated at low levels in vivo during transcription from the Ubiquitin $\mathrm{B}$ locus by molecular misreading. The resulting mutant protein has been shown to inhibit proteasome function. To elucidate causative effects and neuropathological consequences of $\mathrm{UBB}^{+1}$ accumulation, we used a $\mathrm{UBB}^{+1}$ expressing transgenic mouse line, that models UPS inhibition in neurons and exhibits behavioral phenotypes reminiscent of Alzheimer's disease (AD). In order to reveal affected organs and functions, young and aged $\mathrm{UBB}^{+1}$ transgenic mice were comprehensively phenotyped for more than 240 parameters. This revealed unexpected changes in spontaneous breathing patterns and an altered response to hypoxic conditions. Our findings point to a central dysfunction of respiratory regulation in transgenic mice in comparison to wildtype littermate mice. Accordingly, $\mathrm{UBB}^{+1}$ was strongly expressed in brainstem regions of transgenic mice controlling respiration. These regions included, for example, the medial part of the nucleus of the tractus solitarius and the lateral subdivisions of the parabrachial nuclei. In addition, $\mathrm{UBB}^{+1}$ was also strongly expressed in these anatomical structures of AD patients (Braak stage \#6) and was not expressed in non-demented controls. We conclude that long-term UPS inhibition due to $\mathrm{UBB}^{+1}$ expression causes central breathing dysfunction in a transgenic mouse model of $A D$. The $\mathrm{UBB}^{+1}$ expression pattern in humans is consistent with the contribution of bronchopneumonia as a cause of death in AD patients. 


\section{Introduction}

In eukaryotic cells the function of complex protein networks is maintained by highly conserved pathways that regulate the selective degradation of proteins in a spatially and temporally organized manner. For example, the ubiquitin-proteasome system (UPS) contributes considerably to protein quality control together with the autophagy-lysosomal system. The UPS relies on the tagging of proteins destined for breakdown by the attachment of polyubiquitin chains and the subsequent translocation and degradation by proteasomes. The coupling of polyubiquitin chains to proteins is mediated by a cascade of E1, E2 and E3 proteins (Hershko et al., 1983), where specificity is brought about by a large number of E3 ligases (>600). The complexity of the UPS is further increased by various deubiquitination enzymes (>90), the length and linkage type of the polyubiquitin chains, as well as by several ubiquitin-like proteins. In neuronal cells the UPS is required for both preand postsynaptic processes (Fonseca et al., 2006; Trujillo et al., 2010). As a consequence, axonal outgrowth, long-term potentiation, and memory formation are critically dependent on a functional UPS (Yi and Ehlers, 2007).

Mutations in UPS genes have been associated with the pathogenesis of neurodegenerative and other brain diseases. For example, defects in the E3 ligase Park2 are causative for familiar forms of Parkinson's disease (PD) (Kitada et al., 1998). Loss-of-function alleles of the deubiquitinase ubiquitin C-terminal hydrolase (UCHL1) are associated with the two most prevalent neurodegenerative diseases, PD and AD (Setsuie and Wada, 2007). Both diseases are also characterized by the presence of Ubiquitin (UBB) in disease-specific aggregates (Mori et al., 1987) and by a partial impairment of the UPS in general (Keller et al., 2000;Goedert, 2001), as is also observed in the elderly where age-related neurodegeneration is most prevalent. Despite the strong correlation between the UPS, UBB-containing cellular deposits and neurodegeneration, little is known about the impact of UPS inhibition on disease onset or progression. However, the presence of a mutant form of ubiquitin $\mathrm{B}$, the $\mathrm{UBB}^{+1}$ protein, in disease-specific aggregates (e.g. neurofibrillary tangles (NFTs) and intranuclear inclusions) of patients suffering from tauopathies and polyglutamine diseases (Fischer et al., 2003;De Pril et al., 2004) may point to a causative role of UPS dysfunction in these diseases rather than being a consequence. UBB ${ }^{+1} \mathrm{mRNA}$ 
is formed at a low level (Gerez et al., 2005) during transcription from the Ubiquitin B (UBB) locus by a process called "molecular misreading" (Van Den Hurk et al., 2001). Dinucleotide deletions $(\triangle G U)$ in the UBB transcripts occur just next to a GAGAG motif and result in a +1 reading frame shift and an altered protein sequence (van Leeuwen et al., 1998). The resulting $\mathrm{UBB}^{+1}$ protein has a 20 -residue extension and lacks the $\mathrm{C}$-terminal glycine residue required for conjugation to target proteins for degradation. In the elderly, $\mathrm{UBB}^{+1}$ protein levels are known to increase. At higher concentrations $\mathrm{UBB}^{+1}$ is known to inhibit the proteasome (van Tijn et al., 2007) and may therefore contribute to disease progression. In line with these results, $\mathrm{UBB}^{+1}$ accumulates in the hallmarks of all types of $A D$ patients (van Leeuwen et al., 1998; Van Leeuwen et al., 2000; Van Leeuwen et al., 2006). UBB ${ }^{+1}$ transgenic (tg) mice (line \#3413) overexpress human $\mathrm{UBB}^{+1}$ specifically in neurons of the postnatal brain, with strongest neuronal expression in the forebrain (Fischer et al., 2009). In keeping with this, these mice have spatial and contextual memory deficits (Fischer et al., 2009). $\mathrm{UBB}^{+1} \mathrm{tg}$ mice are currently the only mammalian in vivo model for long-term proteasome inhibition in the brain. However, in contrast to AD patients no overt neuronal atrophy or degeneration has been observed in these mice. The expression of cerebral cortical proteins implicated in energy metabolism was altered, thus resembling AD in some aspects (Fischer et al., 2009). Consequently, because the central nervous system is a major regulator of body function, $\mathrm{UBB}^{+1}$ expression in brain might also indirectly affect the functioning of other organ systems. To further explore this, we extended our previous analysis of $\mathrm{UBB}^{+1} \operatorname{tg}$ mice by a systematic phenotyping analysis in the German Mouse Clinic (GMC), which has been successfully used to discover novel and additional phenotypes (Beckers et al., 2009;Enard et al., 2009). Most interestingly, this approach revealed a respiratory dysfunction that was mirrored by transgenic $\mathrm{UBB}^{+1}$ expression in brainstem nuclei involved in breathing control. A similar distribution of $\mathrm{UBB}^{+1}$ expression was also observed in the same brain areas of $A D$ patients. In addition to hippocampal transgene expression in line 3413 , we found significant but mild changes in behavior and metabolic parameters of $\mathrm{UBB}^{+1}$ transgenic mice, which may be additional consequences of $\mathrm{UBB}^{+1}$ expression in brainstem areas (Heneka et al., 2006). These data are consistent with human anatomical data reporting that tangle formation appears early in subcortical 
brain areas such as the locus coeruleus (LC) (Parvizi et al., 2001;Braak and Del Tredici, 2011;Braak et al., 2011).

\section{Materials and methods}

\subsection{Animal model and systematic phenotyping}

$\mathrm{UBB}^{+1}$ overexpressing transgenic mice ( $\mathrm{UBB}^{+1} \mathrm{tg}$, line 3413, 008833C57BI/6.Tg(CaMK2 $\alpha$ UBB) 3413, Jackson \#008833)) (Fischer et al., 2009) were kept on a C57BL/6J background and heterozygous transgenic mice were analyzed further. Non-transgenic littermates were used as controls. To ensure comparability among individuals, all mice were kept under standard animal housing conditions (e.g., bedding, specified pathogen free (SPF) conditions, light/dark cycle, climate control) with food and water ad libitum. Mice were analyzed according to the primary systemic phenotyping approach in the German Mouse Clinic at two different ages of approximately 3 months and 12 months as described below and elsewhere (Gailus-Durner et al., 2005). Secondary phenotyping screens to extend our primary findings were carried out at additional ages as described in the manuscript. Respiratory functions are presented in detail because these were the most significant effects identified by the primary screening protocol.

\subsection{Respiratory function}

Spontaneous breathing patterns of male and female mice (UBB ${ }^{+1}$ tg mice and controls) were studied at the ages of 3 ( $n=6$ for all four groups), 12 ( $n=6$ for all four groups) and 18 months ( $n=8$ for male tg mice and controls; females were not analyzed). For the analysis of lung function and of the ventilatory response to hypercapnic and hypoxic conditions males at the age of 18 months were studied $(n=8)$. A detailed description of the whole body plethysmography and the assessment of the lung function are provided in the supplementary methods. Statistical analyses were performed using a commercially available statistics package (Statgraphics ${ }^{\circledR}$, Statistical Graphics Corporation, Rockville, $M D$ ). Differences between groups were evaluated by Student's t-test. Statistical 
significance was assumed at $p<0.05$. Data are presented as mean values \pm standard error of the mean (SEM).

\subsection{Immunohistochemistry of mouse and human tissues}

Eight 3-month-old, four 6-month-old, five 15-month-old male and four 18-month old UBB ${ }^{+1}$ tg mice and three 3-month-old and three 15-month-old male control mice were given deep pentobarbital anaesthesia (i.p.) and were perfused intracardially with phosphate-buffered saline (PBS) followed by PBS containing 4\% (w/v) paraformaldehyde $(\mathrm{pH}=7.4)$. Dissected brains were sectioned either coronally or sagittally in $50 \mu$ m thick sections with a Vibratome. Sections were stained with anti-UBB ${ }^{+1}$ antibody (Ubi3 16/09/97, final dilution 1:1000) (Fischer et al., 2003). As controls for Ubi3 immunoreactivity the Ubi3 antiserum was adsorbed with ${ }^{6} \mathrm{His}$ tag $\mathrm{UBB}^{+1}$ and the pre-immune control serum was used (other controls were reported previously, (Fischer et al., 2009)). In addition, an anti-14-3-3 zeta antibody ( $\zeta$, \#1001) was generously supplied by A. Aitken (Edinburgh, UK) and tested on brain sections of $\mathrm{UBB}^{+1} \mathrm{tg}$ mice (final dilution 1:500). After the primary antibody incubation the sections were incubated with a biotinylated donkey anti-rabbit antibody followed by avidinbiotin-peroxidase and 3, 3'-diaminobenzidine (DAB) intensified by $0.2 \%$ nickel ammonium sulphate $(\mathrm{pH}=7.6)$. Photographs were made using the dotSlide BX51 microscope (Olympus, Japan).

Human postmortem tissue from AD patients and non-demented controls was obtained from the Radboud University Medical Centre (Department of Pathology, Nijmegen, The Netherlands, see Table 1). Brain tissues were fixed for 1 month after which the unembedded tissue was cut on a Vibratome in $50 \mu \mathrm{m}$ thick sections. Sections were rinsed in TBS and subsequently treated with a graded series of methanol $(20,40,60,80$ and 100\%, 10 minutes each), and back to $20 \%$ methanol with $0.3 \% \mathrm{H}_{2} \mathrm{O}_{2}$ for 30 minutes and for 1 hour in TBS. Sections were then incubated overnight at $4^{\circ} \mathrm{C}$ with antibodies against misfolded tau (MC1, 1:100, and $\mathrm{UBB}^{+1}$ (Ubi2A, 180398, 1:500). All dilutions were in 0.05 $\mathrm{M}$ Tris buffered saline (0.15 M with $0.25 \mathrm{M}$ gelatin and $0.5 \%$ Triton $\mathrm{X}-100, \mathrm{pH}=7.4)$ (Fischer et al., 2003; Van Leeuwen et al., 2006). Subsequently, sections were incubated with donkey-anti-mouse or donkey-anti-rabbit antibodies (Jackson Laboratories), both 
biotinylated (1:400) and ABC (Vector, 1:400) and subsequently stained with DAB as described above.

Table 1: Clinico-pathological information of non-demented controls and AD patients

\begin{tabular}{|c|c|c|c|c|c|c|c|} 
Number & $\begin{array}{c}\text { Age } \\
\text { (years) }\end{array}$ & $\begin{array}{c}\text { Sex } \\
(\mathrm{f} / \mathrm{m})\end{array}$ & $\begin{array}{c}\text { Braak } \\
\text { stage* }\end{array}$ & \multicolumn{2}{c}{$\begin{array}{c}\text { NFTs Brainstem** } \\
\text { LC Amyloid* }\end{array}$} & $\begin{array}{c}\text { Brain } \\
\text { weight } \\
(\mathrm{g})\end{array}$ \\
\hline 1 & 52 & $\mathrm{~m}$ & 0 & + & - & 0 & 1424 \\
\hline 2 & 59 & $\mathrm{f}$ & 0 & + & - & 0 & 1523 \\
\hline 3 & 87 & $\mathrm{~m}$ & 3 & ++ & - & sparse & 1245 \\
\hline 4 & 68 & $\mathrm{~m}$ & 5 & +++ & ++ & frequent & 1312 \\
\hline 5 & 63 & $\mathrm{~m}$ & 6 & +++ & ++ & frequent & 980 \\
\hline 6 & 64 & $\mathrm{f}$ & 6 & +++ & ++ & frequent & 1250 \\
\hline
\end{tabular}

All specimens were fixed in $4 \%$ buffered formalin for 6 weeks

* based upon examination of temporal cortex and hippocampus

** based upon MC1 staining for misfolded tau

$L C=$ locus coeruleus, $P B N I=$ lateral parabrachial nucleus

- = no immunoreactivtity of NFTs or neuropil threads (NTS)

$+=$ minor immunoreactivity of NFTs or NPS

$++=$ considerable immunoreactivity of NFTs or NPs

$+++=$ intense immunoreactivity of NFTs or NPS

\section{Results}

\subsection{Systematic phenotyping in the German Mouse Clinic}

The primary phenotyping protocol in the GMC included the measurement of more than 240 parameters in the areas of allergy, behavior, bone and cartilage, cardiovascular diseases, clinical chemistry, energy metabolism, eye development and vision, immunology, respiratory function, molecular phenotyping, neurology, nociception, and pathology (Gailus-Durner et al., 2005;Gailus-Durner et al., 2009;Fuchs et al., 2011). Because we 
expected that some mutant phenotypes might be associated with the aging process, $\mathrm{UBB}^{+1}$ transgenic (tg) mice and control animals were analyzed for phenotypes in 2 cohorts at the ages of about three and twelve months. Mice of both age cohorts were analyzed in the primary screening protocol within 8 weeks of the modal age. Significant phenotypic differences between $\mathrm{UBB}^{+1} \operatorname{tg}$ and wildtype (WT) control mice that are presented here were found for respiratory function. In addition, we observed significantly altered parameters in metabolism, behavior and hippocampal gene expression, which are described in the supplementary information.

\subsection{Spontaneous breathing pattern}

Spontaneous breathing patterns were measured at rest and at activity, i.e., during calm sitting and short periods of sleeping and while animals were exploring the test chamber, respectively. Data were analyzed for each gender separately. At three months of age significant genotype-dependent respiration differences between control mice and $\mathrm{UBB}^{+1} \mathrm{tg}$ mice were detected for male mice during activity. In this group, the respiratory rate in $\mathrm{UBB}^{+1}$ $\operatorname{tg}$ mice was $8 \%$ higher mostly due to a shorter expiratory time (Figure 1A, S4). In addition, a slightly increased relative duration of inspiration was also observed in male $\mathrm{UBB}^{+1} \operatorname{tg}$ mice compared to WT mice ( $6 \%$ increase, $\mathrm{p}<0.001$ ). The spontaneous breathing of $\mathrm{UBB}^{+1} \mathrm{tg}$ mice was even more affected at the age of 12 months when respiratory timing was significantly altered in male $\mathrm{UBB}^{+1} \operatorname{tg}$ mice during activity and at rest (Figure 1B-C). The trend towards longer time of expiration and shorter relative duration of inspiration during aging was consistent among $\mathrm{UBB}^{+1} \operatorname{tg}$ males (Figure 1B, C and Figure S5) and females (Figure S1, S5) in comparison to the respective WT control groups. At 12 months of age, male $\mathrm{UBB}^{+1} \operatorname{tg}$ mice exhibited significantly shorter relative duration of inspiration at rest $(90 \pm 3 \%$, Figure 1B) and during activity (94 $\pm 3 \%$, Fig. 1C) compared to male WT control mice (set to 100\%). Since the inspiratory time and the mean inspiratory flow rate were not significantly affected in male $\mathrm{UBB}^{+1} \operatorname{tg}$ mice (Figure S5), the observed shorter relative duration of inspiration values were primarily due to an elevated expiratory time. The mean expiratory flow rate was significantly lower at rest $(83 \pm 4 \%)$ and activity $(91 \pm 4 \%)$ in $\mathrm{UBB}^{+1}$ expressing tg mice compared to the corresponding male control group (set to $100 \%$ ), which is most likely due to the differences in the expiratory time (Figure 1B, C). Thus, overall the 
spontaneous breathing pattern of male $\operatorname{tg} \mathrm{UBB}^{+1}$ mice was affected by a statistically significant increase of the expiratory time over age. In contrast, the male WT control group had a tendency towards reduced expiratory time over age. Female tg mice at 12 months of age and male $\mathrm{tg}$ mice at 18 months of age showed similar trends, which became significant for the latter $(p<0.05$; Figure S1, S2). Absolute values for the spontaneous breathing parameters are provided in the supplementary Figures S4, S5 and S6.

The altered spontaneous breathing pattern of the $\mathrm{UBB}^{+1} \operatorname{tg}$ mice is not a mutant phenotype that causes obvious or severe suffering of the mice. The $\mathrm{UBB}^{+1} \operatorname{tg}$ mice appear externally healthy and it is a mutant phenotype that is not apparent by gross inspection of the mice. However, within the primary lung screen of the GMC, which assesses the spontaneous breathing pattern by whole body plethysmography, we rarely observe such a consistent and clear mutant phenotype at least among mouse lines that externally appear rather normal.

To exclude the possibility that the observed differences in breathing patterns between transgenic and WT mice were merely caused by altered lung function per se, we tested various lung function parameters, including lung volume, mechanics, and gas exchange. None of the measured parameters were altered in $\mathrm{UBB}^{+1} \operatorname{tg}$ mice compared to the WT control mice (Figure S3), suggesting that differences in breathing pattern are not due to lung dysfunction per se but rather due to changes in respiratory regulation.
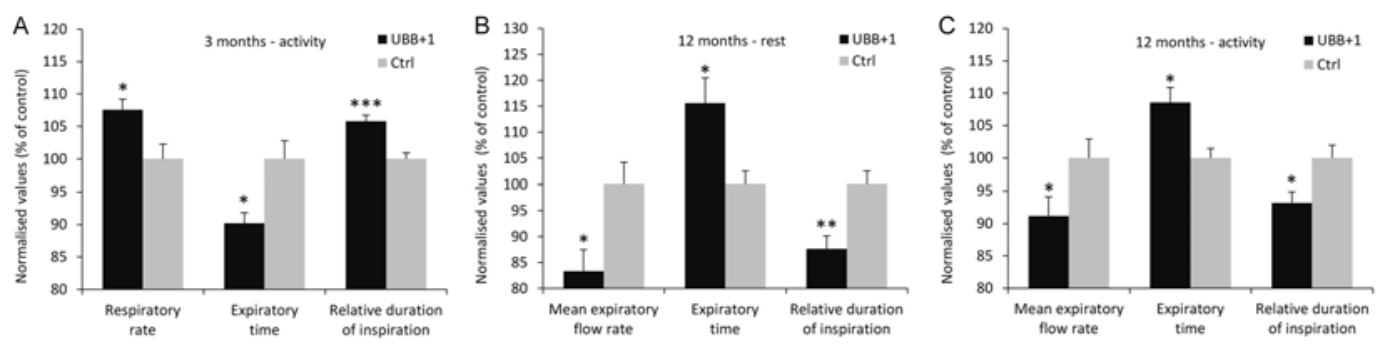

Figure 1: $U B B^{+1}$ tg spontaneous breathing patterns measured by whole body plethysmography. Male $U B B^{+1}$ tg (black bars) and control mice (grey bars) at the age of $(A)$ three months and ( $B$ and $\left.C\right) 12$ months were analyzed ( $n=6$ for all groups). (A) Shows the respiratory rate (f), expiratory time (Te), and relative duration of inspiration (Ti/TT) for male mice under activity. ( $B$ and $C$ ) Show the mean expiratory flow rate (MEF), expiratory time (Te), and the relative duration of inspiration (Ti/TT) at the 
age of 12 months at rest and under activity, respectively. The mean values for the indicated parameters of the control group were set to $100 \%$ and significance by Student's t-test is indicated by ${ }^{*} p<0.05 ;{ }^{* *} p<0.01,{ }^{* * *} p<0.001$; error bars represent SEM.

\subsection{Breathing response to hypoxic and hypercapnic conditions}

The breathing pattern of vertebrates with lung respiration is tightly controlled by the partial pressures of carbon dioxide and oxygen in the blood. Peripheral and central chemoreceptors ensure that increased concentrations of blood carbon dioxide (hypercapnia) or decreased levels of blood oxygen (hypoxia) result in increased respiratory efforts. To test the functionality of these feedback loops and to further examine the relevance of the observed changes in the spontaneous breathing pattern, 18 month-old $\mathrm{UBB}^{+1} \operatorname{tg}$ and control mice were exposed to hypercapnic or hypoxic conditions.

Under hypoxic conditions the most prominent response in all mice of the transgenic as well as the control group was observed for the tidal volume (Figure 2B), which increased about 1.5-fold with $8 \% \mathrm{O}_{2}$ in the respiratory air in comparison to normal air (about $21 \% \mathrm{O}_{2}$ ). When hypoxic conditions were combined with hypercapnic conditions $\left(3 \% \mathrm{CO}_{2}\right.$ in the respiratory air) the tidal volume further increased almost 2-fold. The combined challenge of hypercapnic and hypoxic conditions also increased the relative duration of inspiration by about $170 \%$ (Figure 2D), indicating that it took the animals more time to inhale the increased tidal volume. All values returned to baseline conditions within a 15-minute recovery period. Even though $\mathrm{UBB}^{+1} \operatorname{tg}$ animals and control mice showed a similar increase in tidal volume under hypoxic conditions at $8 \% \mathrm{O}_{2}$, they showed significant differences in two other parameters under this condition. Thus, the respiratory rate in $\mathrm{UBB}^{+1} \mathrm{tg}$ mice declined to about $75 \%$ at $8 \% \mathrm{O}_{2}\left(206.7 \pm 8.3 \mathrm{~min}^{-1}\right)$ whereas this parameter was only slightly changed in control animals (about 90\%; 246.8 \pm 8.4 min $^{-1} ; \mathrm{p}<0.001$; Figure 2A). Secondary to this, the minute ventilation was also significantly lower in $\mathrm{UBB}^{+1} \operatorname{tg}$ mice (Figure $2 \mathrm{C}$; $\mathrm{p}<0.01$ ). Interestingly, when adding the hypercapnic challenge of $3 \% \mathrm{CO}_{2}$ in the breathing air to the hypoxic challenge, the breathing patterns of $\mathrm{UBB}^{+1} \operatorname{tg}$ and controls were not significantly different (Figure 2A, C). In addition, we examined breathing patterns of mice exposed to $3 \%, 5 \%$ and $8 \% \mathrm{CO}_{2}$ in the air (at normal oxygen conditions). Under these conditions, we did not observe differences between $\mathrm{UBB}^{+1} \operatorname{tg}$ animals and WT mice (Figure 
S7). These findings support the notion that the hypoxic response is primarily affected in $\mathrm{UBB}^{+1} \mathrm{tg}$ mice. In contrast, the feedback loop for $\mathrm{CO}_{2}$ levels is apparently not affected in $\mathrm{UBB}^{+1} \operatorname{tg}$ mice.

\section{A. Respiratory rate}

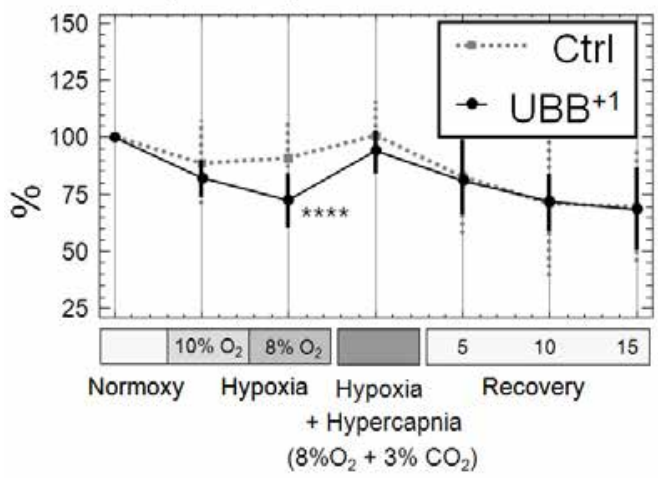

C. Minute ventilation

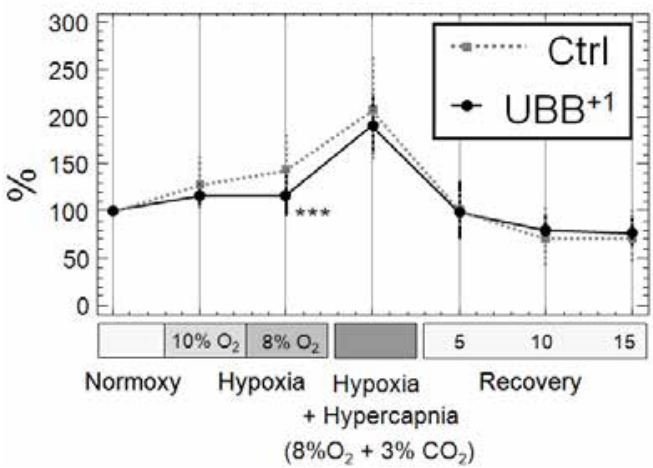

B. Tidal volume

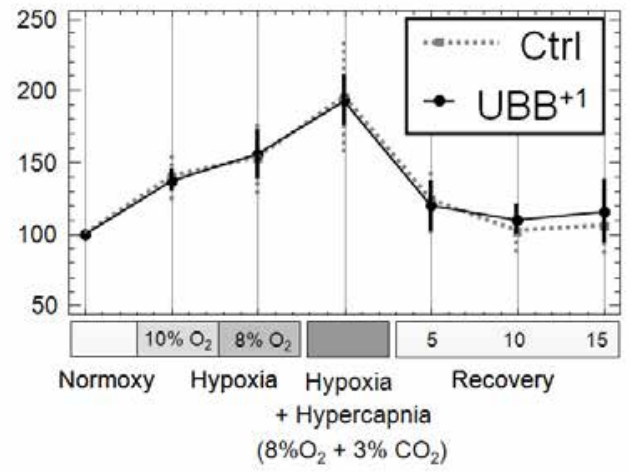

D. Relative duration of inspiratory time

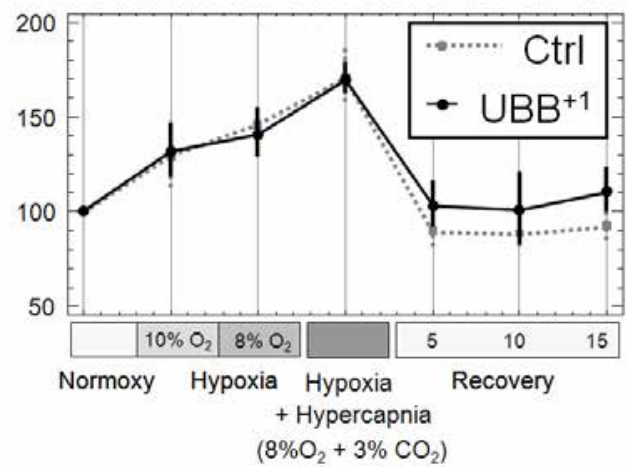

Figure 2: Ventilatory responses to hypoxic and hypercapnic conditions are affected by $\mathrm{UBB}^{+1}$ expression. Male mice at the age of 18 months ( $n=8$ for each group) were exposed to $10 \%$ and $8 \%$ $\mathrm{O} 2$ (hypoxia) followed by a combined exposure of 8\% 02+3\% CO2 (hypoxia and hypercapnia) each for 7 minutes and a recovery period of 15 minutes. (A) Shows the respiratory rates (f), (B) shows the tidal volume (TV), (C) shows the minute ventilation (MV), and (D) shows the relative duration of inspiration time (Ti/TT). Values were calculated relative to baseline levels (set to 100\%) and significance by Student's t-test is indicated by ${ }^{* * * *} p<0.001,{ }^{* * *} p<0.01$; error bars represent SEM. The response to hypercapnic conditions is shown in Supplementary Figure S7. 


\subsection{Anatomic localization of $\mathrm{UBB}^{+1}$ in the mouse brain}

The expression of $\mathrm{UBB}^{+1}$ in brain sections of $\mathrm{UBB}^{+1} \operatorname{tg}$ mice was examined by using the $U_{B B}^{+1}$-specific Ubi3 antibody (bleeding 16-9-97) and a more sensitive procedure (avidinbiotin method) than previously used (Fischer et al., 2009). In the present study hippocampal fibers in the CA region were positive, especially in the CA2 region (Figure 3A, B). The intense fiber staining in the region where the CA3 borders the CA2 appears to reflect an increased fiber density. The incubation of mouse brain tissue with ${ }^{6} \mathrm{His}$ tag $\mathrm{UBB}^{+1}$ and the pre-immune control serum resulted in an absence of immunopositive staining (data not shown). There was no staining in the brains of WT control mice.

The present immunohistochemical staining procedure also enabled a superior anatomic localization of $\mathrm{UBB}^{+1}$ in other brain regions as compared to our previously published results (Fischer et al., 2009). In particular, in the brainstem of $\mathrm{UBB}^{+1} \mathrm{tg}$ mice labeling with the Ubi3 antibody was prominent in regions associated with known functions in respiratory modulation (Figure $3 \mathrm{C}-\mathrm{H}$ ). Both in the dorsal respiratory group (Figure $3 \mathrm{C}-\mathrm{F}$ ) and the pneumotaxic center (Figure $3 \mathrm{G}-\mathrm{H}$ ) $\mathrm{UBB}^{+1}$ immunoreactive nuclei were found. In $\mathrm{UBB}^{+1} \mathrm{tg}$ animals, $\mathrm{UBB}^{+1}$ was present in specific areas of the dorsal vagal complex, such as the area postrema (AP), the nucleus of the tractus solitarius (NTS), and the dorsal motor nucleus of the vagus nerve (Figure $3 \mathrm{C}-\mathrm{F}$ ). Strongly positive $\mathrm{UBB}^{+1}$ immunoreactive neurons were also present in the LC and subnuclei of the parabrachial nucleus (PBN) (Figure 3G, H), especially in the external lateral (PBNel) and dorsal lateral (PBNdl) subnuclei, which receive visceral inputs from the caudal NTS. The rodent external lateral nucleus is involved in respiratory function and in the modulation of respiratory responses to hypoxia and hypercapnia (Song and Poon, 2009a;b). In particular the central respiratory control of the response to hypoxia was affected in $\mathrm{UBB}^{+1} \mathrm{tg}$ mice (Figure 2). These findings are consistent with the hypothesis that highly elevated $\mathrm{UBB}^{+1}$ expression might have mild toxic effects that could interfere with the normal neuronal function (van Tijn et al., 2007). Mice were analyzed for $\mathrm{UBB}^{+1}$ expression at the ages of 3, 6, 15 and 18 months, but no differences between the four ages were found with respect to $\mathrm{UBB}^{+1}$ immunohistochemical staining, which is in agreement with previously published data (Fischer et al., 2009). 


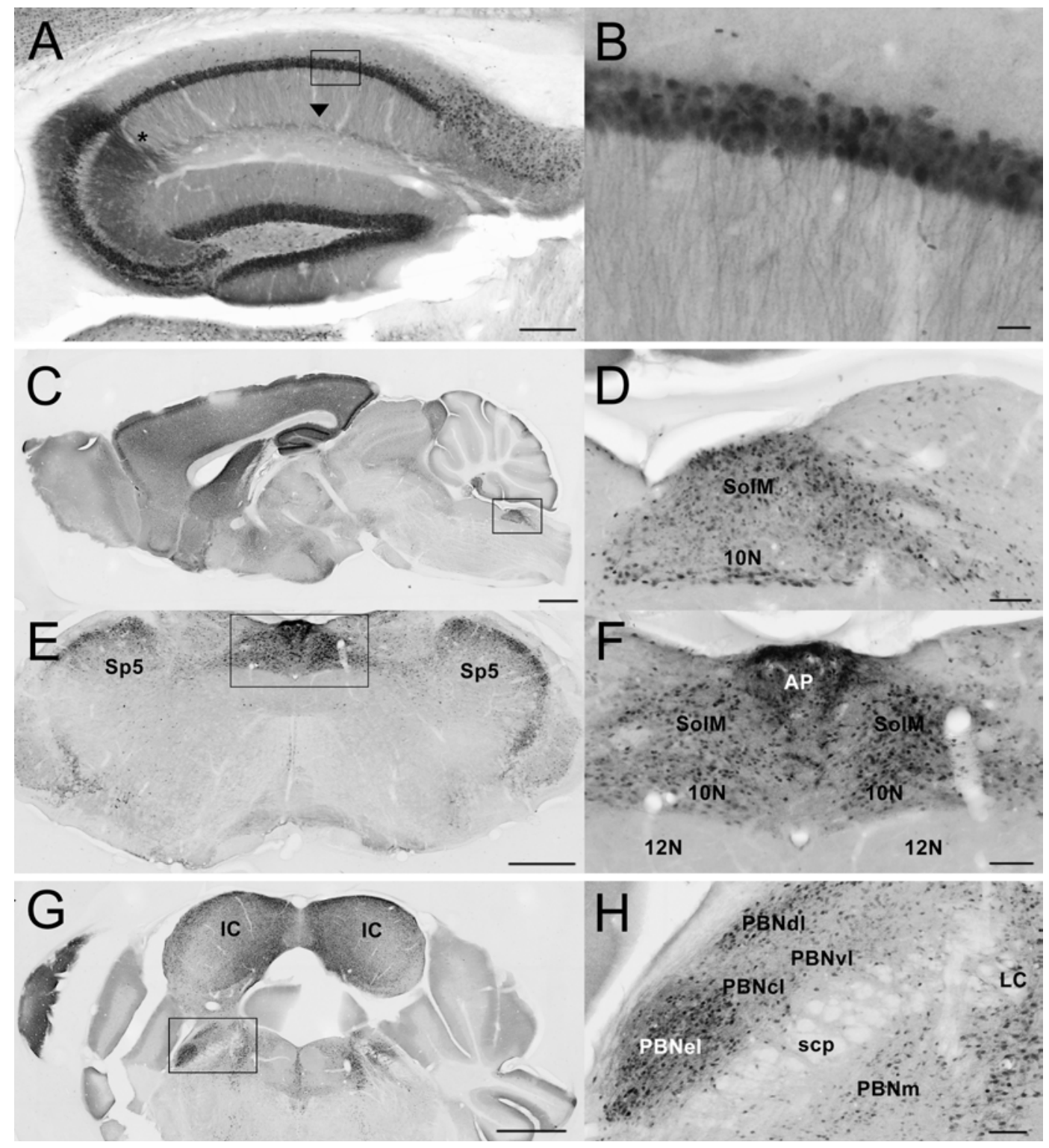

Figure 3: Immunohistochemical expression of $U B B^{+1}$ in the brain of tg mice. $U B B^{+1}$ staining of $(A-B)$ hippocampus and (C-H) brainstem of male $U_{B B}^{+1}$ tg mice at the age of 15 months (hippocampus) and 3 months (brainstem) with the Ubi3 antibody. Sagittal section, A-B) Hippocampal cells are immunoreactive for $U B B^{+1}$. The fibers widespread in the hippocampus are also stained (see boxed area in A), especially in the region where the CA3 borders the CA2 (asterisk). Another region of more intense $U_{B B}^{+1}$ staining reflects the stratum lacunosum (arrowhead), apparently with crossing fibers 
of the perforant pathway. Sagittal $(C, D)$ and coronal $(E-H)$ sections of $U B B^{+1}$ tg mice were incubated with Ubi3. (C) Note the expression of $U B B^{+1}$ in the dorsal vagal complex (nucleus tractus of the solitarius, area postrema, dorsal motor nucleus of vagus nerve). (D) Region of dorsal vagal complex as indicated by the boxed area in (c) at a higher magnification. (E) Coronal section shows again the expression of $U_{B B^{+1}}$ in the dorsal vagal complex and $(F)$ shows this region (boxed area in $E$ ) at a higher magnification. Note in $(E) \cup B B^{+1}$ immunostaining in the spinal 5 nucleus. (G) Note the expression of $\mathrm{UBB}^{+1}$ in neurons of the different subnuclei of the parabrachial nucleus (boxed area). (H) Photograph of parabrachial nucleus at a higher magnification. Nomenclature based upon Fulwiler and Saper (1984). Abbreviations see paper. Scales: $A=200 \mu \mathrm{m}, B=20 \mu \mathrm{m}, C=1 \mathrm{~mm}, D=100 \mu \mathrm{m}, E=$ $500 \mu \mathrm{m}, \mathrm{F}=100 \mu \mathrm{m}, \mathrm{G}=1 \mathrm{~mm}, \mathrm{H}=100 \mu \mathrm{m}$.

\subsection{Anatomic localization of $\mathrm{UBB}^{+1}$ in the human brain}

In the brainstem of sporadic AD patients (Braak stage \#6 and less prominent in stages \#3 and \#5) the same nuclei as in the $\mathrm{UBB}^{+1} \operatorname{tg}$ mouse line 3413 mouse were stained positive for misfolded tau and $\mathrm{UBB}^{+1}$ in the pneumotaxic center of the brain (Fig. 4a-g), e.g., in the LC, subcoeruleus, the lateral and medial parabrachial nuclei, the subpeduncalar pigmented nucleus and the sagulum. Many NFTs, kinky and curly fibers can be seen (Figure 4B, F, and $\mathrm{G}$ ). In the dorsal respiratory group $\mathrm{UBB}^{+1}$ immunoreactivity was found in the nuclei surrounding the solitary tract (Figure $4 \mathrm{H}-\mathrm{I}$ ). These stainings were not observed in stages $\# 0$. Also in the medulla various nuclei around the solitary tract were immunoreactive for misfolded tau (Figure 4H-J) and $\mathrm{UBB}^{+1}$ (Figure 4K-L). These anatomical data support the possibility of lung respiratory dysfunction in AD. 


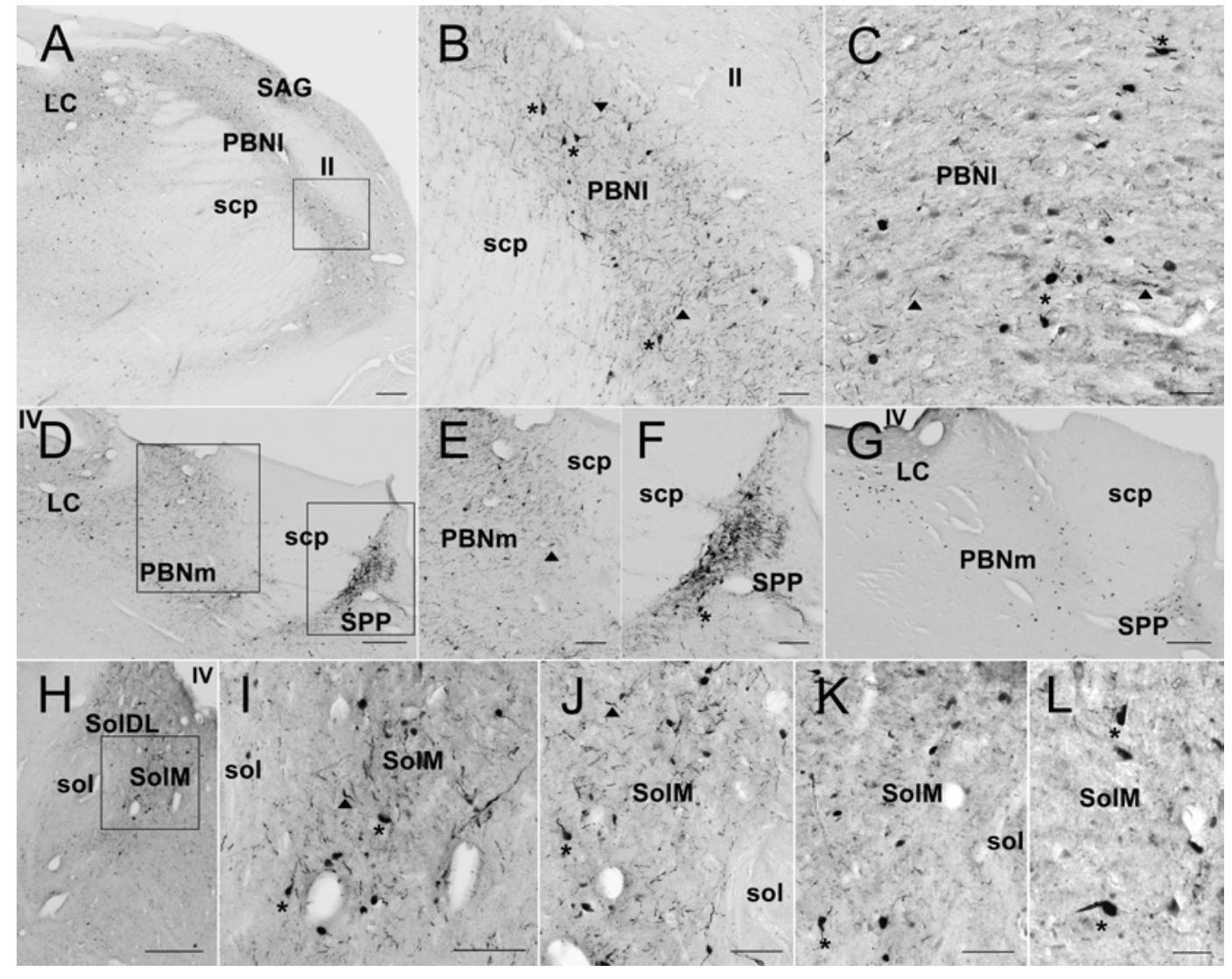

Figure 4: Immunohistochemical expression for misfolded tau and $U B B^{+1}$ in the human brainstem. Intense staining (MC1) for misfolded tau $(A, B, D, E, F, H, I, J)$ and $U B B^{+1}(C, G, K, L)$ in various nuclei of the brainstem of patient \#5 (Braak stage 6). (A-C) Oral pons (Obex $\pm 25 \mathrm{~mm}$, (Paxinos et al., 2012)) and (D-G) Oral pons (Obex $\pm 30 \mathrm{~mm}) . B, E$ and $F$ are larger magnifications of $A$ and $D$ respectively, as indicated by the boxes in A and D. NFTs $\left(^{*}\right)$ and neuropil threads (NTs; $\left.\mathbf{\Delta}\right)$ are stained in the locus coeruleus ( $L C)$, the medial (PBNm) and lateral parabrachial nucleus (PBNI). The $P B N I$ is located between the superior cerebellar peduncle (scp) and the lateral lemniniscus (II). The $P B N m$ and subpeduncular pigmented nucleus (SPP) are located, medially and ventrolaterally respectively, to the scp. (H-L) More caudally in the medulla (Obex $\pm 5 m m$ ) the ventral (SolV), dorsolateral (SoIDL), other solitary subnuclei and the dorsal motor nucleus of the vagus (10N) show NFTs $(H-J)$, that are $U B B^{+1}$ positive $(K-L)$. (I) is a larger magnification of the boxed area in $(H)$. Note brown neuromelanin inclusions in $H-L$. SAG= sagulum, sol= solitary tract. Scales $A, D, G, H=500 \mu m$;

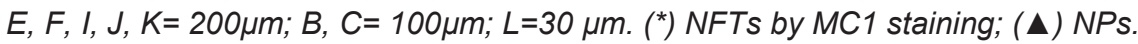




\section{Discussion}

The precise role of the UPS in neurodegeneration and aging is not clear, but the partial impairment of its function in the mouse brain results in spatial memory deficits and changes in fear-related behavior (Fischer et al., 2009; Mardirossian et al., 2009; Hung et al., 2010). In the present study, we extended these data using additional behavioral tests on $\mathrm{UBB}^{+1} \mathrm{tg}$ mice (see also supplementary data) and report further phenotypic differences. Moreover, the intensity of the $\mathrm{UBB}^{+1}$ staining was remarkably enhanced by the improved immunohistochemical staining protocol enabling better localizations (Figure 3A-B). Our data suggest that neurons involved in the central control of respiration are sensitive to longterm UPS inhibition via the expression of the $\mathrm{UBB}^{+1}$ protein. Although the observed differences were significant, the postnatal transgenic expression of $\mathrm{UBB}^{+1}$ seems to be rather well tolerated by mice because the vast majority of the more than 240 parameters measured in the primary phenotyping protocol of the GMC did not show significant differences in comparison to control animals. This limited transgenic phenotype is in accordance with previous studies (Fischer et al., 2009) where no signs of protein aggregations, neurodegeneration or other histological changes were observed. This finding might be explained by the rather modest reduction of proteasomal activity (at least 20\%) in brain tissue of $\mathrm{UBB}^{+1} \operatorname{tg}$ mice (Fischer et al., 2009). The $\mathrm{UBB}^{+1} \operatorname{tg}$ mouse line is to our knowledge the only existing in vivo model for the long-term, low level inhibition of the UPS. This feature models the human situation during aging and neurodegeneration, where chronic reduction of the UPS activity is observed (Keller et al., 2000). To approximate the situation in humans, our systematic phenotyping was done with $\mathrm{UBB}^{+1} \operatorname{tg}$ mice at three and twelve months of age. In both age groups the same biological functions, namely respiratory function, behavior and metabolism were affected in the $\mathrm{UBB}^{+1}$ transgenic mice. In contrast to humans, a dramatic increase in the severity of the transgenic phenotype with age was not observed, which is in line with previous results (Fischer et al., 2009) and which may be related to the relatively short life span of mice. 


\section{1 $\mathrm{UBB}^{+1}$ phenotype affects respiratory function}

The key findings of the respiratory function analysis were related to altered breathing patterns particularly in male $\mathrm{UBB}^{+1} \operatorname{tg}$ mice, which affected the respiratory rate and respiratory timing. Respiratory timing was predominantly affected by a reduction of the relative duration of inspiration (Ti/TT) in combination with an increased expiratory time (Te). An affected breathing pattern per se is a rather unspecific finding, which could be due to several factors, (i) altered central breathing control, (ii) impaired sensitivity of peripheral chemoreceptors, (iii) altered respiratory muscle function, or (iv) affected respiratory function per se. To address these different issues, we tested $\mathrm{UBB}^{+1}$ tg mice for respiratory lung function (lung volumes, mechanics, and gas exchange) and the ventilatory response to hypercapnic and hypoxic conditions, respectively. With regard to lung function, the parameters were not different in $\mathrm{UBB}^{+1} \operatorname{tg}$ and WT mice. These findings indicated that the increased expiratory time is not related to an increased airway resistance, that the gas exchange function of the respiratory system per se (iv) is not affected by $\mathrm{UBB}^{+1}$ transgenic overexpression and that the alterations in breathing pattern are not caused by impaired respiratory muscle function (iii). This conclusion is further supported by the hypercapnic and hypoxic challenge responses. A similar increase up to a factor of 2 in tidal volume in controls and $\mathrm{UBB}^{+1} \operatorname{tg}$ mice was observed indicating that $\mathrm{UBB}^{+1} \operatorname{tg}$ animals have normal thoracic muscle strength. To further distinguish between central (i) and peripheral (ii) chemoreceptor sensitivity, mice were exposed to hypercapnic and/or hypoxic conditions and the ventilatory response was measured. The results show that the hypercapnic response was not affected in $\mathrm{UBB}^{+1} \operatorname{tg}$ mice, while the hypoxic response was. $\mathrm{UBB}^{+1} \mathrm{tg}$ mice showed declining respiratory rates with increasing levels of hypoxia, being $16.3 \%$ lower than in controls at $8 \% \mathrm{O}_{2}$. As a result, minute ventilation was also significantly lower in $\mathrm{UBB}^{+1} \operatorname{tg}$ mice. Interestingly, breathing differences between $\mathrm{UBB}^{+1} \mathrm{tg}$ and control mice were virtually absent when the hypercapnic challenge of $3 \% \mathrm{CO}_{2}$ was added to the hypoxic challenge. This supports the conclusion that primarily the hypoxic feedback loop is affected in $\mathrm{UBB}^{+1} \operatorname{tg}$ mice. Hence, these experimental results suggest that an altered central breathing control (i) is the most likely explanation for the observed phenotype. 
In support of this hypothesis, we observed strong $\mathrm{UBB}^{+1}$ tg expression in various brainstem nuclei which are involved in the regulation of respiratory function. For example, the NTS, which is part of the dorsal respiratory group, was $\mathrm{UBB}^{+1}$ immunoreactive in all the different subnuclei. The caudal part of this nucleus receives afferent information from baroreceptors, mechanoreceptors and from central and peripheral chemoreceptors. Furthermore, it has been shown that the AP together with NTS project to the PBN (Herbert et al., 1990), which comprises a second respiratory center positive for $\mathrm{UBB}^{+1}$ expression. Specifically, the external lateral and dorsal lateral PBN subnuclei were strongly $\mathrm{UBB}^{+1}$ immunoreactive. It has been reported that the external lateral PBN subnucleus in rodents has an important role in the respiratory response to hypoxic and hypercapnic conditions (Song and Poon, 2009a;b). This is of particular interest because $U_{B B}^{+1} \operatorname{tg}$ mice showed differences in their response to hypoxia compared to the controls. Interestingly, it has previously been shown that the LC area and the PBN are among the early targets of the AD-related cytoskeletal pathology, which suggests that parts of the brainstem respiratory centers are affected during AD progression (Rüb et al., 2001;Braak and Del Tredici, 2011). $\mathrm{UBB}^{+1} \operatorname{tg}$ mice are not the only mouse model with altered respiration. For example, tau-P301L transgenic mice show a breathing phenotype, which can be linked to tau (Mapt) expression in defined nuclei of the brainstem such as the Kölliker-Fuse nucleus (Dutschmann et al., 2010). Apart from respiratory dysfunction, swallowing impairments are common in $A D$ patients and frequently lead to aspiration pneumonia (Humbert et al., 2010). The three phases of swallowing (oral, pharyngeal and esophageal) are regulated by central pattern generators in the brainstem and one of them is the NTS (Lang, 2009). This suggests that there might be a functional link between the respiratory changes exhibited by the $\mathrm{UBB}^{+1}$ tg mice and the respiratory and swallowing dysfunctions in $A D$ patients because similar anatomical regions are affected as we have shown in our examination of the distribution of $\mathrm{UBB}^{+1}$ in the brains of $A D$ patients. Intriguingly, it was also recently shown that the minute volume in $A D$ patients under peak exercise conditions is reduced in comparison to a non-demented control group (Billinger et al., 2011). Our data suggest that this change in respiratory response in $A D$ patients could also be due to a dysfunction of central breathing control. 


\section{Concluding remark}

Our comprehensive phenotyping approach revealed a respiratory phenotype in $\mathrm{UBB}^{+1} \mathrm{tg}$ mice, which prompted us to perform an extensive immunohistochemical $\mathrm{UBB}^{+1}$ analysis of the brainstem of these mice. In several areas involved in respiratory regulation such as the lateral parabrachial nuclei (Song and Poon, 2009a;b) and the dorsal vagal complex, we detected intense $\mathrm{UBB}^{+1}$ immunoreactivity. Our data suggest that also in other AD mouse models respiratory function analysis needs to be addressed.

Because $\mathrm{UBB}^{+1}$ tg mice are an animal model for early $A D$ we subsequently analyzed $\mathrm{UBB}^{+1}$ expression in $A D$ patients and healthy individuals. In the human brainstem $\mathrm{UBB}^{+1}$ immunoreactivity and misfolded tau were seen in similar areas as in $\mathrm{UBB}^{+1}$ tg mice and they may well contribute to the selective vulnerability of the brainstem to AD (Parvizi et al., 2001). A role of the lateral parabrachial nucleus in mediating the feedback control of inspiratory drive has been reported (Song and Poon, 2009a). The dorsal respiratory group communicates with this area and shows $\mathrm{UBB}^{+1}$ immunoreactivity as well. It could well be that breathing dysfunction may play a major role in cognitive, behavioral and emotional impairments in early onset AD (Hilaire et al., 2010). Also, a disproportional contribution of bronchopneumonia as a cause of death in $A D$ patients is consistent with our findings that indicate breathing dysfunction as a contributing factor. With respect to respiratory function in aged patients and during $A D$ per se, it has been noted that increased cardiorespiratory fitness in early-stage $A D$ is associated with reduced brain atrophy as compared with nondemented individuals (Burns et al., 2008). Moreover, it was recently reported that aerobic exercise has positive effects on mild cognitive impairment (e.g. (Baker et al., 2010)), underscoring the need to enhance respiratory function. Together with these published observations our data might suggest that the examination of the respiratory system could be an important parameter for monitoring the progression of $A D$. It remains to be analyzed whether other cognitive disorders also display $\mathrm{UBB}^{+1}$ expression and neuronal pathology in central breathing control regions. 


\section{References}

Baker, L.D., Frank, L.L., Foster-Schubert, K., Green, P.S., Wilkinson, C.W., Mctiernan, A., Plymate, S.R., Fishel, M.A., Watson, G.S., Cholerton, B.A., Duncan, G.E., Mehta, P.D., and Craft, S. (2010). Effects of aerobic exercise on mild cognitive impairment: a controlled trial. Archives of Neurology 67, 71-79.

Beckers, J., Wurst, W., and De Angelis, M.H. (2009). Towards better mouse models: enhanced genotypes, systemic phenotyping and envirotype modelling. Nature Reviews: Genetics 10, 371-380.

Billinger, S.A., Vidoni, E.D., Honea, R.A., and Burns, J.M. (2011). Cardiorespiratory response to exercise testing in individuals with Alzheimer's disease. Arch Phys Med Rehabil 92, 20002005.

Braak, H., and Del Tredici, K. (2011). The pathological process underlying Alzheimer's disease in individuals under thirty. Acta Neuropathol. 121, 171-181.

Braak, H., Thal, D.R., Ghebremedhin, E., and Del Tredici, K. (2011). Stages of the Pathologic Process in Alzheimer Disease: Age Categories From 1 to 100 Years. Journal of Neuropathology \& Experimental Neurology 70, 960-969.

Burns, J.M., Mayo, M.S., Anderson, H.S., Smith, H.J., and Donnelly, J.E. (2008). Cardiorespiratory fitness in early-stage Alzheimer disease. Alz. Dis. Assoc. Dis. 22, 39-46.

De Pril, R., Fischer, D.F., Maat-Schieman, M.L., Hobo, B., De Vos, R.A., Brunt, E.R., Hol, E.M., Roos, R.A., and Van Leeuwen, F.W. (2004). Accumulation of aberrant ubiquitin induces aggregate formation and cell death in polyglutamine diseases. Hum. Mol. Genet. 13, 1803-1813.

Dutschmann, M., Menuet, C., Stettner, G.M., Gestreau, C., Borghgraef, P., Devijver, H., Gielis, L., Hilaire, G., and Van Leuven, F. (2010). Upper airway dysfunction of Tau-P301L mice correlates with tauopathy in midbrain and ponto-medullary brainstem nuclei. Journal of Neuroscience 30, 1810-1821.

Enard, W., Gehre, S., Hammerschmidt, K., Holter, S.M., Blass, T., Somel, M., Bruckner, M.K., Schreiweis, C., Winter, C., Sohr, R., Becker, L., Wiebe, V., Nickel, B., Giger, T., Muller, U., Groszer, M., Adler, T., Aguilar, A., Bolle, I., Calzada-Wack, J., Dalke, C., Ehrhardt, N., Favor, J., Fuchs, H., Gailus-Durner, V., Hans, W., Holzlwimmer, G., Javaheri, A., Kalaydjiev, S., Kallnik, M., Kling, E., Kunder, S., Mossbrugger, I., Naton, B., Racz, I., Rathkolb, B., Rozman, J., Schrewe, A., Busch, D.H., Graw, J., Ivandic, B., Klingenspor, M., Klopstock, T., Ollert, M., Quintanilla-Martinez, L., Schulz, H., Wolf, E., Wurst, W., Zimmer, A., Fisher, S.E., Morgenstern, R., Arendt, T., De Angelis, M.H., Fischer, J., Schwarz, J., and Paabo, S. (2009). A humanized version of Foxp2 affects cortico-basal ganglia circuits in mice. Cell 137, 961-971.

Fischer, D.F., De Vos, R.A., Van Dijk, R., De Vrij, F.M., Proper, E.A., Sonnemans, M.A., Verhage, M.C., Sluijs, J.A., Hobo, B., Zouambia, M., Steur, E.N., Kamphorst, W., Hol, E.M., and Van Leeuwen, F.W. (2003). Disease-specific accumulation of mutant ubiquitin as a marker for proteasomal dysfunction in the brain. FASEB Journal 17, 2014-2024.

Fischer, D.F., Van Dijk, R., Van Tijn, P., Hobo, B., Verhage, M.C., Van Der Schors, R.C., Li, K.W., Van Minnen, J., Hol, E.M., and Van Leeuwen, F.W. (2009). Long-term proteasome dysfunction in the mouse brain by expression of aberrant ubiquitin. Neurobiology of Aging 30, 847-863. 
Fonseca, R., Vabulas, R.M., Hartl, F.U., Bonhoeffer, T., and Nagerl, U.V. (2006). A balance of protein synthesis and proteasome-dependent degradation determines the maintenance of LTP. Neuron 52, 239-245.

Fuchs, H., Gailus-Durner, V., Adler, T., Aguilar-Pimentel, J.A., Becker, L., Calzada-Wack, J., Da Silva-Buttkus, P., Neff, F., Gotz, A., Hans, W., Holter, S.M., Horsch, M., Kastenmuller, G., Kemter, E., Lengger, C., Maier, H., Matloka, M., Moller, G., Naton, B., Prehn, C., Puk, O., Racz, I., Rathkolb, B., Romisch-Margl, W., Rozman, J., Wang-Sattler, R., Schrewe, A., Stoger, C., Tost, M., Adamski, J., Aigner, B., Beckers, J., Behrendt, H., Busch, D.H., Esposito, I., Graw, J., Illig, T., Ivandic, B., Klingenspor, M., Klopstock, T., Kremmer, E., Mempel, M., Neschen, S., Ollert, M., Schulz, H., Suhre, K., Wolf, E., Wurst, W., Zimmer, A., and Hrabe De Angelis, M. (2011). Mouse phenotyping. Methods 53, 120-135.

Fulwiler, C.E., and Saper, C.B. (1984). Subnuclear organization of the efferent connections of the parabrachial nucleus in the rat. Brain Research 319, 229-259.

Gailus-Durner, V., Fuchs, H., Adler, T., Aguilar Pimentel, A., Becker, L., Bolle, I., Calzada-Wack, J., Dalke, C., Ehrhardt, N., Ferwagner, B., Hans, W., Holter, S.M., Holzlwimmer, G., Horsch, M., Javaheri, A., Kallnik, M., Kling, E., Lengger, C., Morth, C., Mossbrugger, I., Naton, B., Prehn, C., Puk, O., Rathkolb, B., Rozman, J., Schrewe, A., Thiele, F., Adamski, J., Aigner, B., Behrendt, H., Busch, D.H., Favor, J., Graw, J., Heldmaier, G., Ivandic, B., Katus, H., Klingenspor, M., Klopstock, T., Kremmer, E., Ollert, M., Quintanilla-Martinez, L., Schulz, H., Wolf, E., Wurst, W., and De Angelis, M.H. (2009). Systemic first-line phenotyping. Methods Mol. Biol. 530, 463-509.

Gailus-Durner, V., Fuchs, H., Becker, L., Bolle, I., Brielmeier, M., Calzada-Wack, J., Elvert, R., Ehrhardt, N., Dalke, C., Franz, T.J., Grundner-Culemann, E., Hammelbacher, S., Holter, S.M., Holzlwimmer, G., Horsch, M., Javaheri, A., Kalaydjiev, S.V., Klempt, M., Kling, E., Kunder, S., Lengger, C., Lisse, T., Mijalski, T., Naton, B., Pedersen, V., Prehn, C., Przemeck, G., Racz, I., Reinhard, C., Reitmeir, P., Schneider, I., Schrewe, A., Steinkamp, R., Zybill, C., Adamski, J., Beckers, J., Behrendt, H., Favor, J., Graw, J., Heldmaier, G., Hofler, H., Ivandic, B., Katus, H., Kirchhof, P., Klingenspor, M., Klopstock, T., Lengeling, A., Muller, W., Ohl, F., Ollert, M., Quintanilla-Martinez, L., Schmidt, J., Schulz, H., Wolf, E., Wurst, W., Zimmer, A., Busch, D.H., and De Angelis, M.H. (2005). Introducing the German Mouse Clinic: open access platform for standardized phenotyping. Nature Methods 2, 403404.

Gerez, L., De Haan, A., Hol, E.M., Fischer, D.F., Van Leeuwen, F.W., Van Steeg, H., and Benne, R. (2005). Molecular misreading: the frequency of dinucleotide deletions in neuronal mRNAs for beta-amyloid precursor protein and ubiquitin B. Neurobiology of Aging 26, 145-155.

Goedert, M. (2001). Alpha-synuclein and neurodegenerative diseases. Nature Reviews: Neuroscience 2, 492-501.

Heneka, M.T., Ramanathan, M., Jacobs, A.H., Dumitrescu-Ozimek, L., Bilkei-Gorzo, A., Debeir, T., Sastre, M., Galldiks, N., Zimmer, A., Hoehn, M., Heiss, W.D., Klockgether, T., and Staufenbiel, M. (2006). Locus ceruleus degeneration promotes Alzheimer pathogenesis in amyloid precursor protein 23 transgenic mice. Journal of Neuroscience 26, 1343-1354.

Herbert, H., Moga, M.M., and Saper, C.B. (1990). Connections of the parabrachial nucleus with the nucleus of the solitary tract and the medullary reticular formation in the rat. J. Comp. Neurol. $293,540-580$. 
Hershko, A., Heller, H., Elias, S., and Ciechanover, A. (1983). Components of ubiquitin-protein ligase system. Resolution, affinity purification, and role in protein breakdown. Journal of Biological Chemistry 258, 8206-8214.

Hilaire, G., Voituron, N., Menuet, C., Ichiyama, R.M., Subramanian, H.H., and Dutschmann, M. (2010). The role of serotonin in respiratory function and dysfunction. Respir. Physiol. Neurobiol. 174, 76-88.

Humbert, I.A., Mclaren, D.G., Kosmatka, K., Fitzgerald, M., Johnson, S., Porcaro, E., Kays, S., Umoh, E.O., and Robbins, J. (2010). Early deficits in cortical control of swallowing in Alzheimer's disease. Journal of Alzheimer's disease 19, 1185-1197.

Hung, A.Y., Sung, C.C., Brito, I.L., and Sheng, M. (2010). Degradation of postsynaptic scaffold GKAP and regulation of dendritic spine morphology by the TRIM3 ubiquitin ligase in rat hippocampal neurons. PLoS One 5, e9842.

Keller, J.N., Hanni, K.B., and Markesbery, W.R. (2000). Impaired proteasome function in Alzheimer's disease. J. Neurochem. 75, 436-439.

Kitada, T., Asakawa, S., Hattori, N., Matsumine, H., Yamamura, Y., Minoshima, S., Yokochi, M., Mizuno, Y., and Shimizu, N. (1998). Mutations in the parkin gene cause autosomal recessive juvenile parkinsonism. Nature 392, 605-608.

Lang, I.M. (2009). Brain stem control of the phases of swallowing. Dysphagia 24, 333-348.

Mardirossian, S., Rampon, C., Salvert, D., Fort, P., and Sarda, N. (2009). Impaired hippocampal plasticity and altered neurogenesis in adult Ube3a maternal deficient mouse model for Angelman syndrome. Exp. Neurol. 220, 341-348.

Mori, H., Kondo, J., and Ihara, Y. (1987). Ubiquitin is a component of paired helical filaments in Alzheimer's disease. Science 235, 1641-1644.

Parvizi, J., Van Hoesen, G.W., and Damasio, A. (2001). The selective vulnerability of brainstem nuclei to Alzheimer's disease. Annals of Neurology 49, 53-66.

Paxinos, G., Huang, X.F., Sengul, G., and Watson, C. (2012). "Organization of Human Brainstem Nuclei," in The Human Nervous System, eds. J.G. Mai \& G. Paxinos. 3rd ed (London: Elsevier Academic Press), 260-327.

Rüb, U., Del Tredici, K., Schultz, C., Thal, D.R., Braak, E., and Braak, H. (2001). The autonomic higher order processing nuclei of the lower brain stem are among the early targets of the Alzheimer's disease-related cytoskeletal pathology. Acta Neuropathol. 101, 555-564.

Setsuie, R., and Wada, K. (2007). The functions of UCH-L1 and its relation to neurodegenerative diseases. Neurochem. Int. 51, 105-111.

Song, G., and Poon, C.S. (2009a). Lateral parabrachial nucleus mediates shortening of expiration and increase of inspiratory drive during hypercapnia. Respir. Physiol. Neurobiol. 165, 9-12.

Song, G., and Poon, C.S. (2009b). Lateral parabrachial nucleus mediates shortening of expiration during hypoxia. Respir. Physiol. Neurobiol. 165, 1-8.

Trujillo, G., Nakata, K., Yan, D., Maruyama, I.N., and Jin, Y. (2010). A ubiquitin E2 variant protein acts in axon termination and synaptogenesis in Caenorhabditis elegans. Genetics 186, 135145.

Van Den Hurk, W.H., Willems, H.J., Bloemen, M., and Martens, G.J. (2001). Novel frameshift mutations near short simple repeats. Journal of Biological Chemistry 276, 11496-11498. 
Van Leeuwen, F.W., De Kleijn, D.P., Van Den Hurk, H.H., Neubauer, A., Sonnemans, M.A., Sluijs, J.A., Koycu, S., Ramdjielal, R.D., Salehi, A., Martens, G.J., Grosveld, F.G., Peter, J., Burbach, H., and Hol, E.M. (1998). Frameshift mutants of beta amyloid precursor protein and ubiquitin-B in Alzheimer's and Down patients. Science 279, 242-247.

Van Leeuwen, F.W., Fischer, D.F., Kamel, D., Sluijs, J.A., Sonnemans, M.A., Benne, R., Swaab, D.F., Salehi, A., and Hol, E.M. (2000). Molecular misreading: a new type of transcript mutation expressed during aging. Neurobiology of Aging 21, 879-891.

Van Leeuwen, F.W., Van Tijn, P., Sonnemans, M.A., Hobo, B., Mann, D.M., Van Broeckhoven, C., Kumar-Singh, S., Cras, P., Leuba, G., Savioz, A., Maat-Schieman, M.L., Yamaguchi, H., Kros, J.M., Kamphorst, W., Hol, E.M., De Vos, R.A., and Fischer, D.F. (2006). Frameshift proteins in autosomal dominant forms of Alzheimer disease and other tauopathies. Neurology 66, S86-92.

Van Tijn, P., De Vrij, F.M., Schuurman, K.G., Dantuma, N.P., Fischer, D.F., Van Leeuwen, F.W., and Hol, E.M. (2007). Dose-dependent inhibition of proteasome activity by a mutant ubiquitin associated with neurodegenerative disease. Journal of Cell Science 120, 1615-1623.

Yi, J.J., and Ehlers, M.D. (2007). Emerging roles for ubiquitin and protein degradation in neuronal function. Pharmacological Reviews 59, 14-39. 



\section{CHAPTER 4}

Accumulation of basic amino acids at mitochondria dictates the cytotoxicity of aberrant ubiquitin.

R.J. Braun*, C. Sommer*, C. Leibiger*, R.J. Gentier*, V.I. Dumit, K. Paduch, T. Eisenberg, L. Habernig, G. Trausinger, C. Magnes, T. Pieber, F. Sinner, J. Dengjel, F.W. Van Leeuwen, G. Kroemer and F. Madeo, Cell Reports.2015;10(9):1557-1571

${ }^{*}$ Co-first author

Supplementary material available at:

http://dx.doi.org/10.1016/j.celrep.2015.02.009 


\section{Abstract}

Neuronal accumulation of $\mathrm{UBB}^{+1}$, a frameshift variant of ubiquitin $\mathrm{B}$, is a hallmark of Alzheimer disease (AD). How $\cup_{B B}^{+1}$ contributes to neuronal dysfunction remains elusive. Here we show that in brain regions of AD patients with neurofibrillary tangles $U_{B B}^{+1}$ coexists with VMS1, the mitochondrion-specific component of the ubiquitin-proteasome system (UPS). Expression of $\mathrm{UBB}^{+1}$ in yeast disturbs the UPS, leading to mitochondrial stress and apoptosis. Inhibiting UPS activity exacerbates, while stimulating UPS by the transcription activator Rpn4 reduces $\mathrm{UBB}^{+1}$-triggered cytotoxicity. High levels of the Rpn4 target protein Cdc48 and its cofactor Vms1 are sufficient to relieve programmed cell death. We identified the $\mathrm{UBB}^{+1}$-induced enhancement of the basic amino acids arginine, ornithine and lysine at mitochondria as a decisive toxic event, which can be reversed by Cdc48/Vms1-mediated proteolysis. The fact that AD-induced cellular dysfunctions can be avoided by UPS activity at mitochondria has potentially far-reaching pathophysiological implications. 


\section{Introduction}

$\mathrm{UBB}^{+1}$, a loss-of-function variant of ubiquitin $\mathrm{B}(\mathrm{UBB})$, accumulates in neurofibrillary tangles (NFTs), a pathological hallmark in AD (van Leeuwen et al., 1998). UBB ${ }^{+1}$ is translated from an aberrant mRNA encoding $\mathrm{a}+1$ frameshift protein in which the $\mathrm{C}$-terminal glycine residue required for ubiquitination is replaced by an extension of 20 amino acids (Dennissen et al., 2010). The detrimental impact of $\mathrm{UBB}^{+1}$ has been studied in neuronal cell cultures, transgenic mice, and yeast (de Vrij et al., 2001;Fischer et al., 2009;Tank and True, 2009). $\mathrm{UBB}^{+1}$ is as a substrate for truncation, ubiquitination, and proteasomal degradation (Lindsten et al., 2002;van Tijn et al., 2007;van Tijn et al., 2010;Dennissen et al., 2011). Whereas the UPS can assure the degradation of low levels of $\mathrm{UBB}^{+1}$, higher levels impair the UPS and subvert the homeostatic mechanisms allowing for its elimination (Lindsten et al., 2002;van Tijn et al., 2007;Fischer et al., 2009;van Tijn et al., 2010). At high levels, $\mathrm{UBB}^{+1}$ affects mitochondrial dynamics and triggers neuronal cell death (de Vrij et al., 2001; Tan et al., 2007) through as yet elusive mechanisms.

Yeast is an established model for studying programmed cell death mechanisms that are often shared with animal cells, including the contribution of caspases and mitochondrionassociated cell death proteins, such as cytochrome c (Carmona-Gutierrez et al., 2010). Yeast models have been used to explore cell killing by neurotoxic proteins, such as Parkinson disease-associated $\alpha$-synuclein, and the outcome could be successfully translated to fly, worm and murine disease models, as well as to human disease (Braun et al., 2010;Büttner et al., 2013).

Driven by these premises, we established a yeast cell death model for $\mathrm{UBB}^{+1}$-triggered neurotoxicity. Our findings revealed that $\mathrm{UBB}^{+1}$ interfered with the UPS, and triggered the perturbation of the mitochondrion-associated basic amino acid synthesis executing cell death. The mitochondrion-associated UPS subroutine, depending on the AAA-ATPase Cdc48 and its co-factor Vms1, strongly antagonized UBB ${ }^{+1}$ cytotoxicity. Since VMS1, the human homolog of yeast Vms1, co-exists with $\mathrm{UBB}^{+1}$ in NFTs, these data imply a potential pivotal role of the UPS at mitochondria in AD. 


\section{Materials and methods}

\subsection{Yeast strains and growth conditions}

Yeast expression constructs, strains and growth conditions were described in Supplemental Experimental Procedures. Gene expression was under the control of galactose-regulated promoters. For stressing cells, cultures were treated for $4 \mathrm{~h}$ with acetate. For stable isotope labeling (SILAC), cells expressing vector controls, $\mathrm{UBB}^{+1}$, or $\mathrm{UBB}^{+1}$ and Vms1 were grown in media supplemented either with Lys0 and Arg0 (normal isotopes), or with Lys4 and Arg6, or with Lys8 and Arg10 (heavy isotopes, Silantes, Munich, Germany).

\subsection{Measuring cytotoxicity based on growth and clonogenicity}

Assays were performed as described in Supplemental Experimental Procedures. Briefly, growth deficits upon expression of proteins of interest on solid or liquid media, as compared to vector controls, suggest for cytotoxic effects of these proteins on (growing) yeast cells. For clonogenic assays, 500 cells from liquid yeast cultures expressing proteins of interest or vector controls, respectively, were plated on agar plates, on which expression is repressed. The number of colonies (colony forming units, CFU) formed after two days of incubation correlates with the fitness of the culture.

\subsection{Measurement of oxidative stress, cell death, apoptosis and necrosis}

Oxidative stress was determined by measuring the conversion of dihydroethidium (DHE, Sigma-Aldrich, Vienna, Austria) to the red fluorescent ethidium applying a fluorescence plate reader or a flow cytometer. Cell death was measured by the incorporation of the 'vital dye' propidium iodide (PI, Sigma-Aldrich) in cells that have lost their plasma membrane integrity using a flow cytometer. Annexin V/PI co-staining (Annexin V-FLUOS Staining Kit, Roche Applied Sciences, Mannheim, Germany) for discriminating early and late apoptosis, as well as necrosis, and terminal deoxynucleotidyl transferase dUTP nick end labeling (TUNEL) for measuring apoptosis (In Situ Cell Death Detection Kit, Roche Applied 
Sciences) were performed by flow cytometry. See Supplemental Experimental Procedures for details.

\subsection{Measurement of cellular oxygen consumption, mitochondrial membrane potential, and cellular ATP levels}

Oxygen consumption of stationary yeast cultures was analyzed using the FireSting optical oxygen sensor system (Pyro Science, Aachen, Germany). The decrease of the oxygen concentration over time in yeast cultures was determined. Mitochondrial membrane potential was assessed with flow cytometry after staining cells with tetramethylrhodamine methyl ester (TMRM, Molecular Probes, Life Technologies), a fluorescent dye that accumulates within mitochondria dependent on their membrane potential. To determine the ATP level of yeast cultures, intracellular metabolites were obtained using hot ethanol extraction. ATP was measured using the ATP Determination Kit (Molecular Probes, Life Technologies). This assay is based on an ATP-dependent reaction of recombinant firefly luciferase, which induces bioluminescence of its substrate D-luciferin and is directly correlated with the ATP content. All data were normalized to the number of living cells within the samples. See Supplemental Experimental Procedures for details.

\subsection{Measurement of UPS activities}

For determining the level of polyubiquitinated proteins in cellular extracts, immunoblots of cellular extracts were incubated with an ubiquitin-specific antibody and immunosignals were quantified with ImageJ $1.47 \mathrm{~m}$. For measuring the turnover of UPS substrates, the ubiquitin-fusion protein ubiquitin-G76V-Green Fluorescent Protein (GFP) was coexpressed with $\mathrm{UBB}^{+1}$ or vector controls. GFP fluorescence (relative fluorescence units, RFU) and optical densities (OD 600 ) were determined using the FLUOstar Omega plate reader. RFU was normalized to $\mathrm{OD}_{600}$, in order to determine the level of ubiquitin-GFP fusion proteins per culture. Measurement of chymotrypsin-like proteasomal activities were performed using the FLUOstar Omega plate reader, applying the luminescence-based Proteasome-Glo ${ }^{\mathrm{TM}}$ Cell-Based Assay (Promega, Heidelberg, Germany). See Supplemental Experimental Procedures for details. 


\subsection{Generation of cell extracts, SDS-PAGE and immunoblot analyses}

Yeast cultures were incubated in expression media (SCGal) for the indicated time points. Cell extracts were generated by pre-treating yeast pellets in $\mathrm{NaOH}$ followed by heating in SDS lysis buffer. Protein extracts were separated on Tricine-SDS polyacrylamide gels, transferred on PVDF membranes and incubated with primary and secondary antibodies coupled to horseradish peroxidase. Immunodetection was done using luminol. Membranes were digitized in an ImageQuant LAS 4000 (GE Healthcare, Munich, Germany). Images were processed with Adobe Photoshop CS6 (Adobe). Immunoblot quantification was done with the gel analysis method in ImageJ $1.47 \mathrm{~m}$. See Supplemental Experimental Procedures for details.

\subsection{Mass spectrometry}

Crude mitochondrial extracts were taken up in SDS lysis buffer, thawed, reduced with DTT and alkylated using iodoacetamide (Sigma-Aldrich). Protein mixtures were separated by SDS-PAGE using Bis-Tris gels (NuPAGE, Invitrogen). The gel lanes were cut into slices, which were in-gel digested with trypsin (Promega), and the resulting peptide mixtures were processed on STAGE tips. Mass spectrometry was performed on a LTQ Orbitrap XL mass spectrometer (Thermo Fisher Scientific, Bremen, Germany) coupled to an Eksigent NanoLC-ultra. See Supplemental Experimental Procedures for details.

\subsection{Metabolomics}

For extraction of metabolites cultures were harvested by filtration, washed with $\mathrm{dd}_{2} \mathrm{O}$ and quenched in liquid nitrogen. Metabolites were extracted by acid extraction using trichloroacetic acid, and by hot ethanol extraction. Extracts obtained from uniformly ${ }^{13} \mathrm{C}$ labeled yeast cells served as internal standard. Metabolites were determined using ion pair reversed-phase liquid chromatography coupled to negative electro spray high resolution mass spectrometry (IP-RP-LC/HRMS). LC/MS measurements were normalized to the total number of cells of each sample. See Supplemental Experimental Procedures for details. 


\subsection{Immunohistochemistry}

Experiments with human materials were in accordance with the local ethical committees at the Universities of Bayreuth (Germany) and Maastricht (The Netherlands). Postmortem tissues of hippocampi from $A D$ patients and non-demented controls were obtained from the Netherlands Brain Bank (Amsterdam, The Netherlands) (Table S6) as paraffin sections. For immunohistochemistry, sections were deparaffinated, incubated with primary antibodies against the indicated proteins, and with biotin-coupled secondary antibodies followed by the avidin-biotin-peroxidase complex. Immunodetection was performed by the colorimetric reaction of 3,3'-diaminobenzidine (DAB). Sections were dehydrated, and coverslipped. See Supplemental Experimental Procedures for details.

\section{Results}

\subsection{Expression of human $\mathrm{UBB}^{+1}$ in yeast recapitulates hallmarks of $\mathrm{UBB}^{+1}$ in neurons}

To investigate whether the introduction of $\mathrm{UBB}^{+1}$ into yeast recapitulates hallmarks of $\mathrm{UBB}^{+1}$ accumulation in neurons, we expressed monomeric ubiquitin $\mathrm{B}$ (UBB), $\mathrm{UBB}^{+1}$, as well as an $\mathrm{UBB}^{+1}$ variant lacking two lysine residues $(\mathrm{K} 29,48 \mathrm{R})$ that are important for its ubiquitination. When expressing UBB, we detected a discrete immunoreactive band at the size of monomeric ubiquitin ( $9 \mathrm{kDa}$ ), and an immunoreactive smear across a wide range of the immunoblot that corresponds to ubiquitinated proteins (Figure 1A). This smear was not detectable upon transformation with $\mathrm{UBB}^{+1}$ or $\mathrm{UBB}^{+1}-\mathrm{K} 29,48 \mathrm{R}$, reflecting their loss-offunction. Instead, $\mathrm{UBB}^{+1}$ or $\mathrm{UBB}^{+1}-\mathrm{K} 29,48 \mathrm{R}$ were detectable as 12 and $9 \mathrm{kDa}$ protein species (full-length and truncated $\mathrm{UBB}^{+1} ; \mathrm{fl}^{-} \mathrm{UBB}^{+1}$ and $\mathrm{tUBB}^{+1}$ ) that accumulated over time (Figures $1 \mathrm{~A}, \mathrm{~S} 1 \mathrm{~A}+\mathrm{B}$ ). In cells expressing $\mathrm{UBB}^{+1}$, a faint higher molecular weight species corresponding to the size of mono-ubiquitinated fl-UBB ${ }^{+1}(21 \mathrm{kDa})$ appeared (Figure 1A, FLAG long exposure, asterisks). Consistent with a role of lysines 29 and/or 48 in the ubiquitination of $\mathrm{UBB}^{+1}$, this band was absent in cells expressing $\mathrm{UBB}^{+1}-\mathrm{K} 29,48 \mathrm{R}$. These results suggest that in yeast human UBB (but not $\mathrm{UBB}^{+1}$ ) can serve as a substrate for ubiquitin ligases and that, like in neurons, $\mathrm{UBB}^{+1}$ is ubiquitinated and truncated. 
Next, we investigated whether $\mathrm{UBB}^{+1}$ expression results in UPS impairment by means of three complementary assays: (i) the measurement of polyubiquitinated endogenous proteins by immunoblot; (ii) the assessment of the abundance of transgenic ubiquitinG76V-GFP, which is a substrate of the ubiquitin-fusion degradation pathway, and (iii) an enzymatic assay designed to quantify the chymotrypsin-like proteasomal activity. Cells expressing $\mathrm{UBB}^{+1}$ or $\mathrm{UBB}^{+1}-\mathrm{K} 29,48 \mathrm{R}$ contained a higher level of polyubiquitinated proteins than cells transformed with vector controls (Figure 1B-C), suggesting decreased UPSdependent protein turnover. The steady-state levels of ubiquitin-G76V-GFP were significantly increased upon expression of $\mathrm{UBB}^{+1}$ or $\mathrm{UBB}^{+1-K 29,48 R}$ (Figure 1D). In

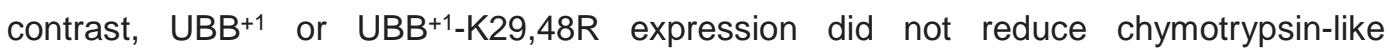
proteasomal activities (Figure S1C). These data suggest that, in yeast like in neurons, $\mathrm{UBB}^{+1}$ expression impairs the UPS. However, in yeast $\mathrm{UBB}^{+1}$ does neither directly affect the enzymatic activity of proteasomes, nor is its ubiquitination essential for UPS dysfunction. 

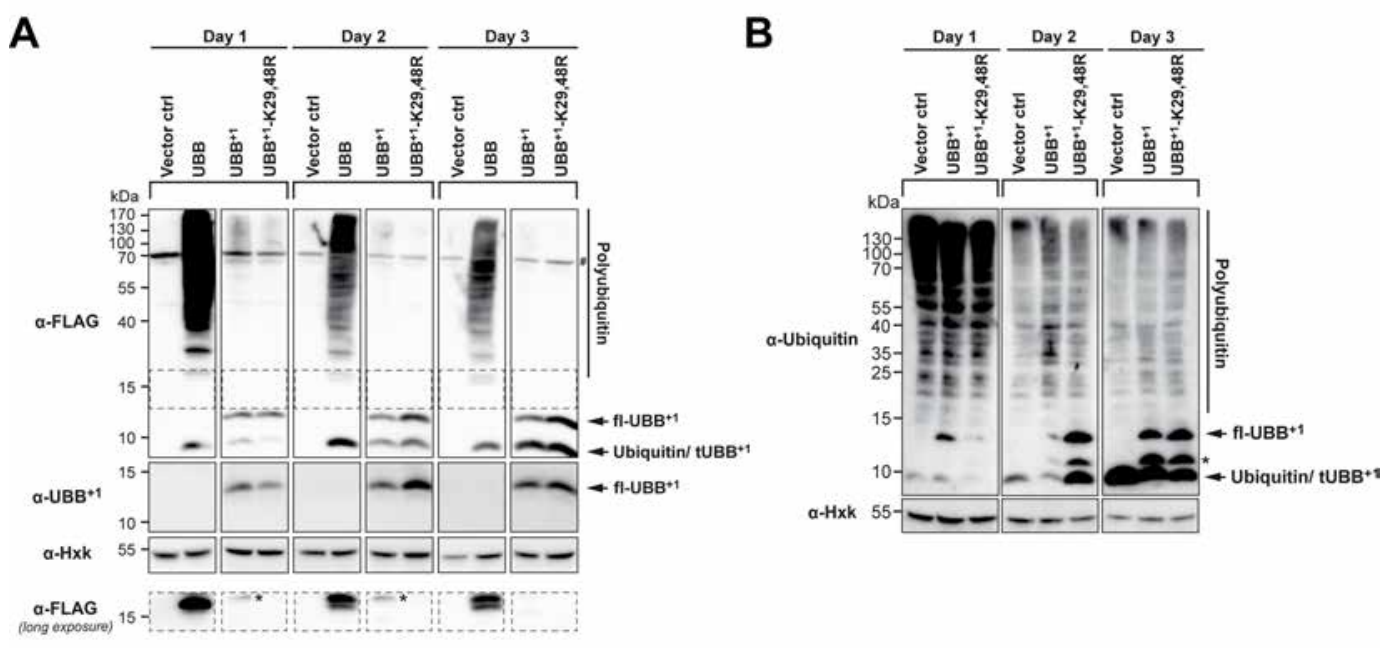

C

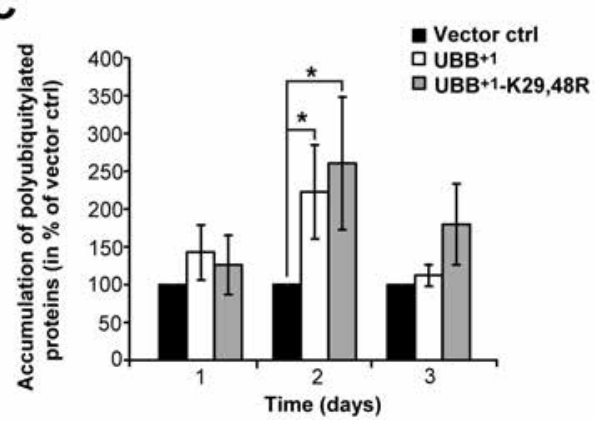

D

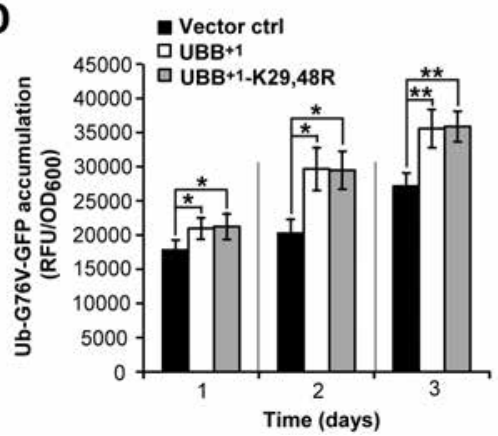

Figure 1: Expression of UBB ${ }^{+1}$ in yeast and its effect on UPS activity. A) Proteins were expressed for 1, 2, or 3 days and determined by immunoblotting of cell extracts using antibodies directed against the N-terminal FLAG-tag, or the specific C-terminus of $U B B^{+1}$. Hexokinase (Hxk) was used as loading control. \#: unspecific protein band, fl-UBB ${ }^{+1}$ : full-length $U B B^{+1}, t U B B^{+1}$ : truncated $U B B^{+1}$. B) The level of polyubiquitinated proteins and of $\mathrm{UBB}^{+1}$ in cell extracts was determined by immunoblotting using an antibody directed against ubiquitin. *: uncharacterized ubiquitin variant. C) Quantification of (B). The levels of polyubiquitinated proteins of cells transformed with vector controls were set to $100 \%$ in every experiment. D) Cellular level of ubiquitin-G76V-GFP upon UBB ${ }^{+1}$ expression. GFP fluorescence (relative fluorescence units, RFU) was normalized to optical densities (OD600). Data: percent change values $(C)$ and mean values $(D)$, respectively. Error bars: standard error. p-values: ${ }^{*} p<0.05,{ }^{* *} p<0.01$. See Table S1 and Figure S1. 


\section{2 $\mathrm{UBB}^{+1}$ triggers oxidative stress and programmed cell death upon protracted expression}

To assess its effects on the fitness of proliferating cells, we performed growth assays on agar plates and in liquid cultures. As a positive control of cytotoxicity, transactive response DNA binding protein 43 (TDP-43), a causal factor for motor neuron degeneration, was expressed. In sharp contrast with TDP-43, UBB ${ }^{+1}$ and $\mathrm{UBB}^{+1}-\mathrm{K} 29,48 \mathrm{R}$ failed to compromise the growth of cells on agar plates (Figure $2 A$ ), and in liquid cultures (Figure $2 \mathrm{~B}$ ), suggesting that $\mathrm{UBB}^{+1}$ is unable to kill proliferating cells.

Next, we studied the effects of $\mathrm{UBB}^{+1}$ or $\mathrm{UBB}^{+1}-\mathrm{K} 29,48 \mathrm{R}$ on chronologically aged cultures. For this, the proportion of viable cells capable of forming a colony (clonogenicity) on nutrient-containing solid medium was studied at different time points following $\mathrm{UBB}^{+1}$ or $\mathrm{UBB}^{+1}-\mathrm{K} 29,48 \mathrm{R}$ expression. Consistent with the growth assays, $16 \mathrm{~h}$ (day 1 ) after $\mathrm{UBB}^{+1}$ or UBB ${ }^{+1-K 29,48 R}$ expression cells exhibited a similar clonogenic potential as did cells expressing vector controls (Figure $2 \mathrm{C}$ ). In contrast, we observed a $10 \%$ and $25 \%$ decrease in clonogenic cell survival when expressing $\mathrm{UBB}^{+1}$ for 2 and 3 days, respectively. Exogenously applied stressors, including acetate and hydrogen peroxide further enhanced the cytotoxicity of prolonged $\mathrm{UBB}^{+1}$ expression (Figure S2A-B left panels). Upon both chronological aging and stress experiments, $U_{B B}^{+1}-K 29,48 R$ turned out to be slightly less cytotoxic as compared to $\mathrm{UBB}^{+1}$ (Figures $2 \mathrm{C}$, S2A-B left panels), suggesting that ubiquitinated $\mathrm{UBB}^{+1}$ is slightly more cytotoxic than $\mathrm{UBB}^{+1}$.

We next examined whether the $\mathrm{UBB}^{+1}$-induced loss of clonogenicity correlated with the manifestation of oxidative stress, which can be detected by the intracellular conversion of the reactive oxygen species (ROS)-sensitive stain DHE to fluorescent ethidium. We observed indistinguishable low levels of oxidative stress after expressing $\mathrm{UBB}^{+1}$ or $\mathrm{UBB}^{+1}$ $\mathrm{K} 29,48 \mathrm{R}$ for $16 \mathrm{~h}$ (day 1 ) (Figure 2D). At later time points, the levels of oxidative stress progressively increased in all cultures with chronological aging, and $\mathrm{UBB}^{+1}$-expressing cells exhibited a mild but significant increase in oxidative stress as compared to vector controls. Thus, upon $\mathrm{UBB}^{+1}$ expression increased markers of oxidative stress coincided with decreased clonogenic cell survival (cf. Figures 2C-D and S2C). When combined with protracted $\mathrm{UBB}^{+1}$ expression, acetate or hydrogen peroxide exacerbated the signs of 
oxidative stress (Figure S2A-B right panels). As shown for clonogenic survival, UBB ${ }^{+1}$ K29,48R demonstrated slightly decreased levels of oxidative stress upon chronological aging or exogenously applied stress as compared to $\mathrm{UBB}^{+1}$ (Figures 2D, S2A-B).

To determine the mode of cell death triggered by the expression of $\mathrm{UBB}^{+1}$ or $\mathrm{UBB}^{+1}$ $\mathrm{K} 29,48 \mathrm{R}$, we performed double staining with Annexin V- Fluorescein isothiocyanate (FITC) and PI. Annexin V-FITC labels externalized phosphatidylserine that appears on the surface of apoptotic cells, whereas $\mathrm{PI}$ is a vital dye that stains cells that have lost plasma membrane integrity during necrosis. Two days after $\mathrm{UBB}^{+1}$ expression the frequencies of early apoptotic (Annexin $\mathrm{V}-\mathrm{FITC}^{+} \mathrm{PI}^{-}$), late apoptotic or secondary necrotic (Annexin $\mathrm{V}-\mathrm{FITC}^{+}$ $\mathrm{PI}^{+}$), and necrotic cells (Annexin V-FITC- $\mathrm{PI}^{+}$) were increased, as compared with vector controls (Figures 2E, S2D). Apoptosis induction by $\mathrm{UBB}^{+1}$ could be confirmed by the TUNEL that detects fragmentation of nuclear DNA (Figures 2F, S2E). Consistent to the results obtained from the clonogenic survival and oxidative stress experiments, UBB ${ }^{+1}$ $\mathrm{K} 29,48 \mathrm{R}$ triggered cell death in a lower number of cells as compared to $\mathrm{UBB}^{+1}$ (Figure 2EF). Altogether, these results indicate that the protracted expression of $\mathrm{UBB}^{+1}$ can induce apoptotic and necrotic killing of yeast cells, and that ubiquitinated $\mathrm{UBB}^{+1}$ is a slightly better killer than $\mathrm{UBB}^{+1}$. 
A

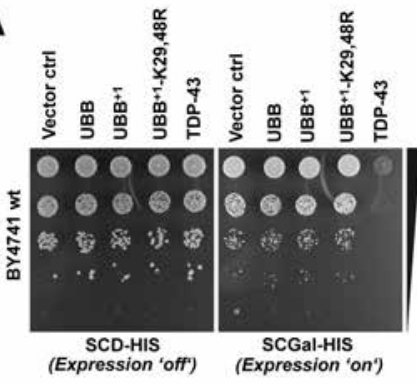

B

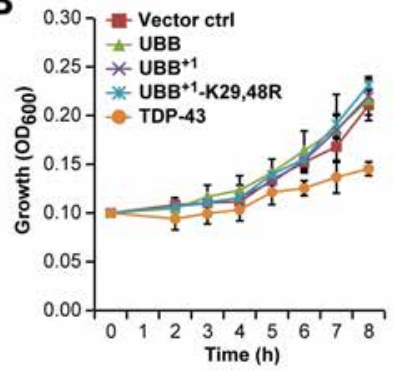

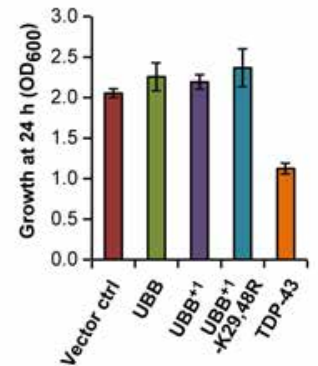

C
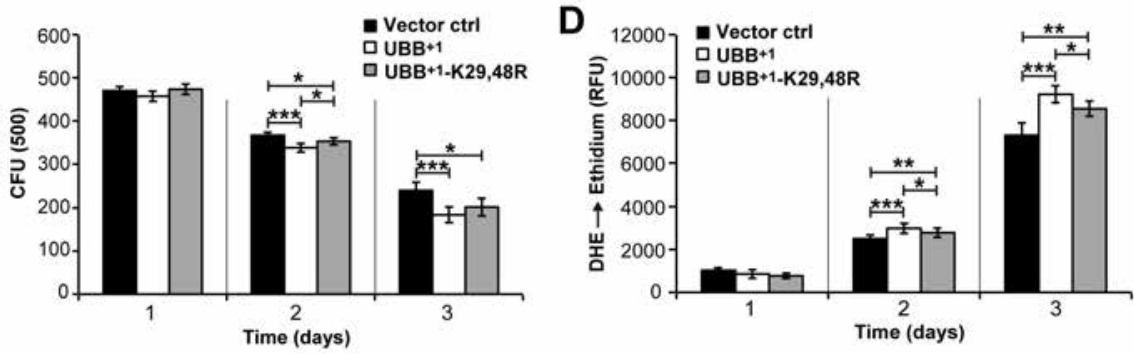

E

$\mathbf{F}$

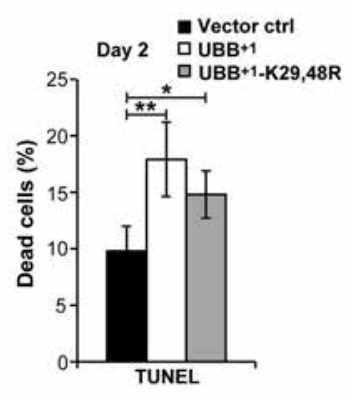

Figure 2: Cytotoxicity and cell death upon $\mathrm{UBB}^{+1}$ expression. A) Growth on solid media. Cultures were spotted in serial dilutions onto solid media inducing or repressing expression. B) Growth in liquid media. Left panel: Growth curves. Right panel: Cell densities during stationary phase. C) Yeast cells were evaluated for clonogenicity (colony forming units, CFU) at the indicated time points after inducing expression. D) Oxidative stress levels (DHE staining) were measured using a fluorescence plate reader at the indicated time points after inducing expression. E-F) Apoptosis and necrosis. E) 2 days after inducing expression, yeast cells were measured for (early) apoptosis (Annexin $V+/ P I-$ ), necrosis (Annexin V-/PI+), and (late) apoptosis/secondary necrosis (Annexin $V+/ P I+)$. F) TUNEL positive cells are referred to be apoptotic. Data: mean values (B-F). Error bars: standard deviation (B), and standard error (C-F), respectively. $p$-values: ${ }^{*} p<0.05,{ }^{* *} p<0.01,{ }^{* * *} p<0.001$. See Table S1 and Figure S2. 


\subsection{The UPS capacity and the ratio of mutant to WT ubiquitin determine $\mathrm{UBB}^{+1}$-triggered cytotoxicity}

To investigate the putative contribution of dysfunctional UPS to $\mathrm{UBB}^{+1}$-triggered cytotoxicity, we measured the cytotoxic potential of $\mathrm{UBB}^{+1}$ in the context of enhanced or suppressed UPS. Since full knockout of genes coding for proteasomal subunits is lethal, yeast strains bearing point mutations in one or two proteasomal genes were employed (Heinemeyer et al., 1993). The chymotrypsin-like proteasomal activity was reduced in strains carrying mutant alleles in the proteasomal subunits Pre1 and Pre 2 by $>88 \%$ (Figure $3 \mathrm{~A}$ ). In these conditions of close-to-complete proteasomal inactivation, significantly reduced clonogenic cell survival was only observed in the pre1-1 and the pre1-1/pre2-2 strains upon $\mathrm{UBB}^{+1}$ expression for day 1 , and in the pre1-1/pre2-2 strain upon $\mathrm{UBB}^{+1}$ expression for day 2 (Figures 3B, S3A), as compared to wild-type (WT) strain. One explanation for the increased $\mathrm{UBB}^{+1}$-triggered cytotoxicity would be that $\mathrm{UBB}^{+1}$ accumulates in these strains due to impaired $\mathrm{UBB}^{+1}$ degradation. However, we could not observe increased steady-state levels of $\mathrm{UBB}^{+1}$ in these strains (neither fl- $\mathrm{UBB}^{+1}$, nor $\mathrm{tUBB}^{+1}$, nor ubiquitinated fl-UBB ${ }^{+1}$ ) (Figures S3D-F, and data not shown). Thus, although severe proteasomal inactivation can increase $\mathrm{UBB}^{+1}$-triggered cell death, there is no strict correlation between the loss of proteasomal capacity on the one hand, and the increase in $\mathrm{UBB}^{+1}$-triggered cytotoxicity or the increase in the steady-state levels of $\mathrm{UBB}^{+1}$ on the other hand.

Next, we measured $\mathrm{UBB}^{+1}$-induced cytotoxicity in knock-out strains lacking selective UPS genes, including (i) UBI4 encoding ubiquitin (Finley et al., 1987), (ii) RPN4 encoding a major transcriptional UPS activator (Mannhaupt et al., 1999), (iii) UBR2 encoding the E3 ligase responsible for Rpn4 degradation (Kruegel et al., 2011), (iv) YUH1 encoding the ubiquitin protease which cleaves fl-UBB ${ }^{+1}$ into tUBB $^{+1}$ (Dennissen et al., 2011), and (v) UBP6 encoding a deubiquitinase, which can be inhibited by extended ubiquitin proteins (Krutauz et al., 2014). Only RPN4 deletion significantly impaired the chymotrypsin-like proteasomal capacity of the cells (Figure 3C). Notably, upon comparable fl- $\mathrm{UBB}^{+1}$ steady-state levels (Figure $\mathrm{S} 3 \mathrm{G}+\mathrm{I}), \mathrm{UBB}^{+1}$-triggered cytotoxicity was significantly increased in $\Delta u b i 4$ as compared to $\Delta r p n 4$ upon both stressed und unstressed conditions (Figures 3D, S3B), 
although the proteasomal capacity was lower in $\Delta r p n 4$ as compared to $\Delta u b i 4$ (Figure $3 C$ ). These data propose that the ratio of mutant ubiquitin $\left(\mathrm{UBB}^{+1}\right)$ to $\mathrm{WT}$ ubiquitin (encoded by $U B / 4)$ is more relevant for determining $\mathrm{UBB}^{+1}$-triggered cytotoxicity than the proteasomal capacity.

Upon stressed conditions, $\mathrm{UBB}^{+1}$-triggered cytotoxicity was markedly increased in $\Delta y u h 1$ as compared to WT cells upon comparable fl- $\mathrm{UBB}^{+1}$ steady-state levels (Figures 3D, S3B, $\mathrm{S} 3 \mathrm{H}-\mathrm{I})$. These data suggest that $\mathrm{UBB}^{+1}$ truncation is a putative protective event, for instance as part of a mechanism to degrade excessive $\mathrm{UBB}^{+1}$. In $\triangle u b p 6$ as compared to WT cells $U_{B B}^{+1}$-triggered cytotoxicity was unaltered upon comparable fl- $\mathrm{UBB}^{+1}$ steady state levels (Figures 3D, S3B, S3G-I), suggesting that Ubp6 activity is not protective against the accumulation of the extended ubiquitin $\mathrm{UBB}^{+1}$.

$\mathrm{UBB}^{+1}$-triggered cytotoxicity was significantly relieved in $\Delta u b r 2$ cells upon stressed conditions (Figures 3D, S3B), in which Rpn4 is stabilized and consequently the UPS activity is increased (Kruegel et al., 2011). Consistently, Rpn4 expression, which also leads to increased UPS activities (Figure 3E), was protective for $\mathrm{UBB}^{+1}$-expressing WT cells (Figures 3F, S3C) but not for cells lacking UBI4 (Figure S3C). In both cases the protective effect cannot be explained by decreased steady-state levels of UBB ${ }^{+1}$ (Figures S3G+I, $\mathrm{S} 3 \mathrm{~J}+\mathrm{K})$. These data show that increasing UPS capacity is protective for $\mathrm{UBB}^{+1}$-expressing cells, but not by affecting the turnover of $\mathrm{UBB}^{+1}$ itself but rather by interrupting the lethal signaling cascade triggered by $\mathrm{UBB}^{+1}$. 
A

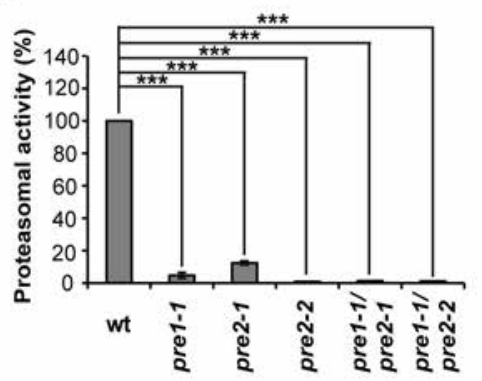

B

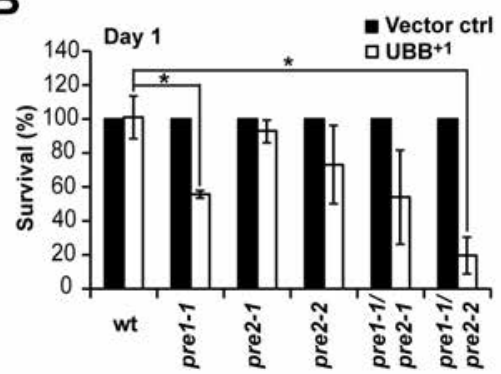

C

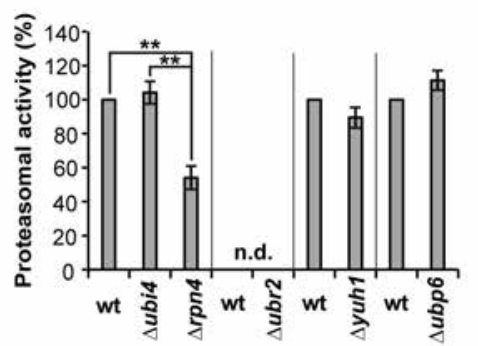

D

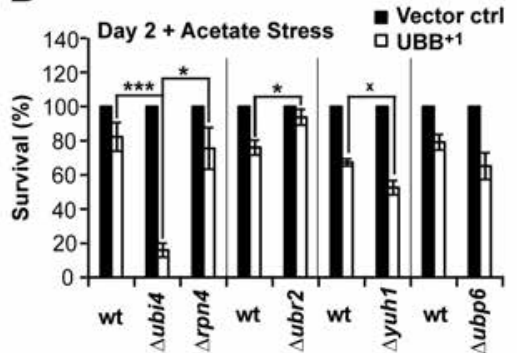

E
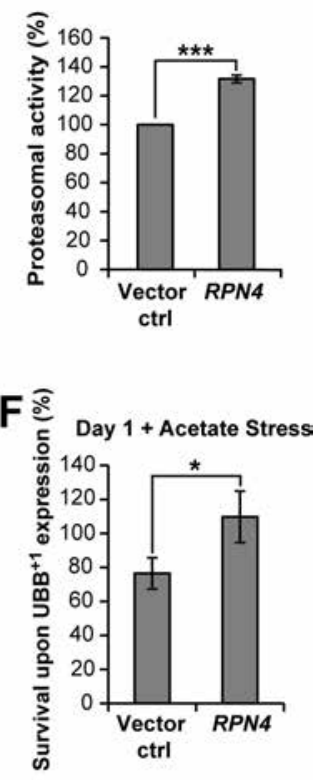

Figure 3: UBB ${ }^{+1}$-triggered cytotoxicity in yeast strains with various UPS capacities. A) Cultures were grown in logarithmic phase in YPD at $30^{\circ} \mathrm{C}$ and chymotrypsin-like activities were determined in proteasomal mutant strains. The relative luminescence units (RLUs) obtained using WT cells were set to $100 \%$ in every experiment. B) Clonogenicity in proteasomal mutant strains 1 day after inducing expression. The CFUs obtained using cells expressing vector controls were set to $100 \%$ in every experiment. C) Cultures were grown in logarithmic phase in YPD at $30^{\circ} \mathrm{C}$ and chymotrypsin-like activities were determined in UPS knock-out strains. The RLUs obtained using WT cells were set to $100 \%$ in every experiment. D) Clonogenicity in UPS knock-out strains 2 days after inducing expression following acetate treatment. The CFUs obtained using cells expressing vector controls were set to $100 \%$ in every experiment. E) Cultures were grown in logarithmic phase in defined medium inducing expression of the transcription activator RPN4, and chymotrypsin-like proteasomal activities were determined. The RLUs obtained using cells carrying vector controls were set to $100 \%$ in every experiment. F) Clonogenicity of $\mathrm{UBB}^{+1}$-expressing cultures in strains with endogenous (vector control) and elevated levels of Rpn4 (Rpn4), respectively. Clonogenicity was determined 1 day after inducing expression followed by acetate treatment. The CFUs obtained using cells with endogenous and elevated levels of Rpn4, respectively, but lacking $U B B^{+1}$, were set to $100 \%$ in every experiment (not shown). Data: percent change values. Error bars: standard error. p-values: xp < 0.1 , ${ }^{*} p<0.05,{ }^{* *} p<0.01,{ }^{* * *} p<0.001$. See Table S1 and Figure S3. 


\section{4 $\mathrm{UBB}^{+1}$ causes lethal mitochondrial dysfunction}

Oxidative stress and mitochondrial impairment are hallmarks of neurotoxin-elicited death in yeast and neurons (Braun, 2012;Debattisti and Scorrano, 2013). Therefore, we analyzed whether oxidative stress, which occurred starting by day 2 of $U_{B B}^{+1}$ expression (Figure 4A), correlated with mitochondrial impairment. Two days after inducing $\mathrm{UBB}^{+1}$ expression, the mitochondrial network was fragmented in both $\mathrm{UBB}^{+1}$-expressing cells, as well as in cells carrying vector controls (data not shown), which is typical for stationary phase cultures. However, after shifting these cultures to fresh growth medium (which represses UBB ${ }^{+1}$ expression) the recovery of the mitochondrial network was significantly compromised in cultures transformed with $\mathrm{UBB}^{+1}$-encoding constructs as compared with vector controls (Figures 4B, S4A). These data suggest that mitochondrial and oxidative stresses coincide in cells expressing $\mathrm{UBB}^{+1}$.

We further tested for mitochondrial impairment by measuring the cellular oxygen consumption, the mitochondrial membrane potential, and the ATP levels in cells expressing $\mathrm{UBB}^{+1}$ for days 2 and 3 . Whereas the cellular oxygen consumption and mitochondrial membrane potential were significantly increased by day 3 in (surviving) cells expressing $\mathrm{UBB}^{+1}$ (Figure 4C-D), the cellular ATP levels were significantly decreased by days 2 and 3 (Figure $4 \mathrm{E}$ ). These data hint to hyperactive mitochondria, which are incapable to prevent a metabolic crisis in $\mathrm{UBB}^{+1}$-expressing cells.

In yeast, alterations in the cytochrome $b c_{1}$ complex of the mitochondrial respiratory chain may contribute to the loss of respiratory capacity and the production of lethal ROS (Eisenberg et al., 2007; Diaz et al., 2012). For instance, loss of the Rieske iron-sulfur protein 1 (Rip1), a key component of the cytochrome $b c_{1}$ complex, results in increased ROS generation and mitochondrial dysfunction (Diaz et al., 2012). Indeed, the cellular level of Rip1 was markedly decreased by days 2 and 3 upon $\mathrm{UBB}^{+1}$ expression as compared with vector controls (Figure S4B-C). Consistently, Rip1 and cytochrome $c$ were depleted in the mitochondrial fraction of $\mathrm{UBB}^{+1}$-expressing cells (Figure $4 \mathrm{~F}-\mathrm{G}$ ). These data further hint to a major $\mathrm{UBB}^{+1}$-induced mitochondrial dysfunction, in which the respiratory chain is impaired (depletion of Rip1 and cytochrome c), leading to the production of ROS (for which cellular oxygen is needed), and the decline of cellular ATP levels. 
Hyperpolarization of mitochondria may precede mitochondrion-dependent yeast death (Eisenberg et al., 2007), therefore, we expressed $\mathrm{UBB}^{+1}$ in strains deleted for genes encoding a range of mitochondrial cell death proteins, including the yeast $\mathrm{BH}$-only protein (Ybh3) that translocates to mitochondria to mediate their permeabilization, and several potentially cytotoxic proteins that can be released from mitochondria such as apoptosisinducing factor 1 (Aif1), endonuclease $\mathrm{G}$ (Nuc1), and the two cytochrome $\mathrm{c}$ isoforms (Cyc1, Cyc7). Deletion of NUC1 resulted in a paradoxical increase in $U_{B B}^{+1}$-triggered cytotoxicity, and loss of Ybh3 did not have any effect upon both stressed and unstressed conditions (Figures 4H, S4D). In contrast, UBB ${ }^{+1}$-mediated cytotoxicity was significantly decreased in strains depleted from isoform 2 of cytochrome $c(\Delta c y c 7)$ upon stressed conditions (Figures $4 \mathrm{H}, \mathrm{S} 4 \mathrm{D}$ ). The steady-state levels of $\mathrm{UBB}^{+1}$ were not decreased in the $\Delta c y c 7$ as compared to WT strain (Figure S4F), and this strain maintained a normal state of respiratory competence (presumably due to the presence of the cytochrome $\mathrm{c}$ isoform 1 Cyc1) (Figure S4I), excluding trivial explanations for the cytoprotective action of $\Delta c y c 7$. Thus, our data suggest the implication of mitochondria in $\mathrm{UBB}^{+1}$-triggered cell death.

Next, we tested for a possible role of the unfolded protein response (UPR) and the ER in $\mathrm{UBB}^{+1}$-triggered cytotoxicity, and expressed $\mathrm{UBB}^{+1}$ for two days in cells lacking the UPR kinase Ire1 and its downstream target Hac1, as well as in cells lacking the ER cell death protease Kex1 (which executes cell death in which mitochondria play a pivotal role (Hauptmann and Lehle, 2008)). Upon stress, $\mathrm{UBB}^{+1}$-triggered cytotoxicity was relieved in $\Delta$ ire 1 and $\Delta k e x 1$ but not in $\Delta$ hac1 cells (Figures $4 \mathrm{I}, \mathrm{S} 4 \mathrm{E}$ ), under conditions where the steady-state levels of $\mathrm{UBB}^{+1}$ were comparable (Figure S4G-H). These data suggest for an implication of the ER in $\mathrm{UBB}^{+1}$-triggered cytotoxicity, but due to the lack of rescue in the $\Delta$ hac1 cells, a critical involvement of the UPR is unlikely. 
A

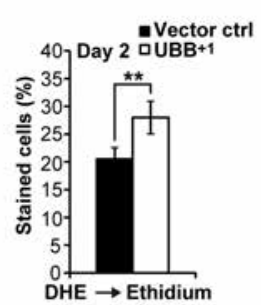

D

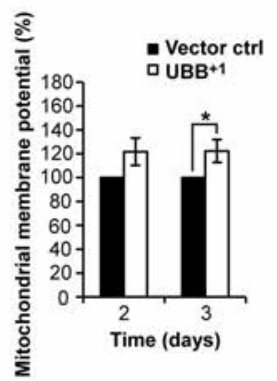

B

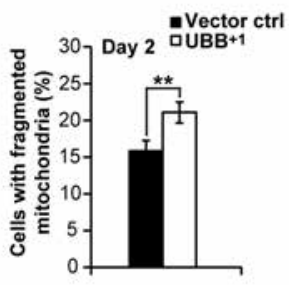

E

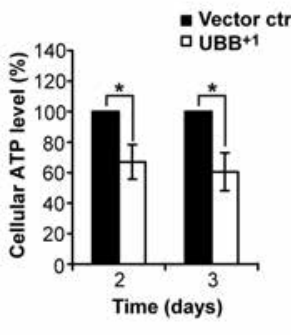

C

F
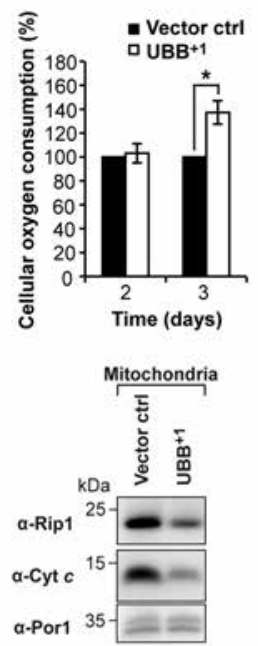

G

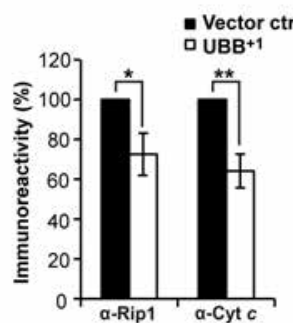

H

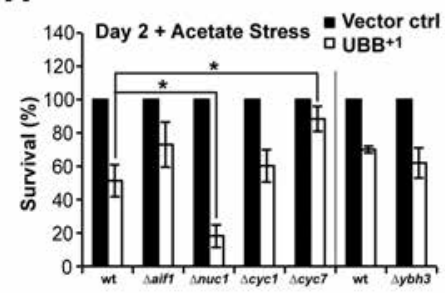

I

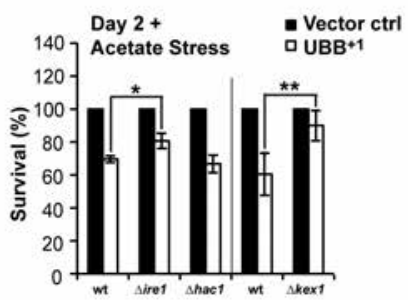

Figure 4: Pivotal mitochondrial impairment upon $\mathrm{UBB}^{+1}$ expression. A) Oxidative stress levels were measured by flow cytometry 2 days after inducing expression. B) Mitochondrial fragmentation. $U B B^{+1}$ and RFP fused with a mitochondrial targeting sequence were expressed. 2 days after induction, cultures were shifted to fresh media repressing expression, and after $3 \mathrm{~h}$ the proportion of cells with fragmented mitochondria was quantified. C-E) Cellular oxygen consumption (C), mitochondrial membrane potential (D), and cellular ATP levels (E) were determined 2 and 3 days after inducing $U B B^{+1}$ expression. The oxygen consumption (C), mitochondrial membrane potential (D), and ATP levels (E) measured using cells carrying vector controls were set to $100 \%$ in every experiment. F-G) Protein alterations in crude mitochondria. $U B B^{+1}$ was expressed for $24 h$ and crude mitochondria were isolated by differential centrifugation. F) Immunoblot demonstrating the steady-state levels of Rip1, cytochrome c (Cyc), and the mitochondrial outer membrane protein Por1 as loading control. (G) Quantification of (F). The immunoreactive signals obtained using cells carrying vector controls were set to $100 \%$ in every strain and experiment. $\mathrm{H}-\mathrm{I}) \mathrm{UBB^{+1 }}$-triggered cytotoxicity in strains deleted from genes encoding mitochondrial cell death $(H)$, and ER-associated proteins (I), respectively. 
Clonogenicity was determined 2 days after inducing expression followed by acetate treatment. The CFUs obtained using cells carrying vector controls were set to $100 \%$ in every experiment. Data: mean values (A-B), and percent change values (C-E, G-I), respectively. Error bars: standard error. p-values: ${ }^{*} p \leq 0.05,{ }^{* *} p<0.01$. See Table S1 and Figure S4.

\subsection{Perturbation of basic amino acid synthesis at mitochondria is a decisive toxic event upon $\mathrm{UBB}^{+1}$ accumulation}

Next, we performed quantitative proteomic analyses of crude mitochondria after SILAC. This approach led to the identification of 16 proteins whose abundance was significantly altered (increased for 10 or decreased for 6 proteins) upon UBB ${ }^{+1}$ expression (Figure 5A, Table S2). Among the proteins with established mitochondrial localization, three were enzymes participating in amino acid metabolism, namely Put1 (involved in proline degradation), Arg5, 6 and Arg8 (involved in arginine and ornithine biosynthesis). In addition, $\mathrm{UBB}^{+1}$ induced the accumulation of the cytosolic enzyme Lys1 (involved in lysine biosynthesis), an increase in the motor protein Myo3 and the (putative) peroxisomal proteins Gpd1 and Str3, in crude mitochondria. Upon acetate stress, deletion of the $A R G 5,6, A R G 8$, and the $L Y S 1$ genes restored the clonogenic potential of $U_{B B}^{+1}$ expressing cells, whereas the deletion of all other genes had no effect (Figures 5B, S5A). These data point to a hitherto unexpected involvement of the biosynthesis of basic amino acids (arginine, ornithine and lysine) in $\mathrm{UBB}^{+1}$-triggered cytotoxicity.

To challenge this hypothesis, we measured the cellular steady-state levels of arginine, ornithine, and lysine in cultures expressing $\mathrm{UBB}^{+1}$ (Figure $5 \mathrm{C}$ ). Indeed, we observed a marked increase in the cellular levels of all three basic amino acids, in particular ornithine, upon $\mathrm{UBB}^{+1}$ accumulation. To weight the contribution of arginine and ornithine (as opposed to their metabolic intermediates) to $\mathrm{UBB}^{+1}$ cytotoxicity, we measured ROS production upon $\mathrm{UBB}^{+1}$ expression in strains depleted from the arginine and ornithine biosynthetic enzymes (Figure 5D). Depletion of all enzymes operating upstream of cytosolic ornithine (Arg2, Arg5, 6, Arg7, and Ort1) significantly relieved $\mathrm{UBB}^{+1}$-triggered cytotoxicity both in unstressed and acetate-stressed conditions (Figures 5E, S5B). In contrast, none of the enzymes downstream of cytosolic ornithine (Arg3, Arg1, and Arg4, which are needed for the conversion of ornithine into arginine) were required for the cytotoxic action of $\mathrm{UBB}^{+1}$. 
Notably, all tested enzymes operating upstream of cytosolic ornithine are mitochondrionassociated (Ljungdahl and Daignan-Fornier, 2012). Therefore, we concluded that $\mathrm{UBB}^{+1}$ triggers the mitochondrion-associated biosynthesis of ornithine, leading to increased cytosolic levels of ornithine (and its product arginine). This plays a decisive role in executing $\mathrm{UBB}^{+1}$-triggered cell death.

If this model is true, increasing cytosolic levels of either ornithine or arginine (which can easily be interconverted into each other) should recover the cytotoxic effect of $\mathrm{UBB}^{+1}$ in strains with interrupted mitochondrion-associated biosynthesis of ornithine. Therefore, we measured $\mathrm{UBB}^{+1}$-triggered cell death in the strain depleted for the mitochondrial protein Ort1 in growth media with increasing concentrations of arginine and ornithine, respectively. It turned out that $\Delta$ ort 1 cells were not able to efficiently uptake ornithine from the growth media, because the severe growth deficit of the $\Delta$ ort1 strain in growth media lacking arginine could not be relieved by increasing concentrations of ornithine in the growth media (data not shown). In contrast, $\Delta$ ort1 cells grew well in the presence of arginine in growth media lacking ornithine (data not shown), demonstrating the efficient cellular uptake of arginine. As expected, yeast cells lacking Ort1 were protected from $\mathrm{UBB}^{+1}$-triggered cell death upon moderate concentrations of arginine (30 and $50 \mathrm{mg} / \mathrm{L}$ ) in the growth media (Figure 5F). In contrast, elevated concentrations of arginine in the growth media (150 and $300 \mathrm{mg} / \mathrm{L}$ ) recovered the cytotoxic effect of $\mathrm{UBB}^{+1}$ (Figure 5F), substantiating the decisive role of increased cellular levels of arginine (and cytosolic ornithine) in executing UBB ${ }^{+1}$ triggered cell death.

In order to address the role of cellular levels of lysine, we measured $\mathrm{UBB}^{+1}$-triggered cell death in the strain depleted from Lys1 in growth media with increasing concentrations of lysine. Whereas deletion of $L Y S 1$ relieved $\mathrm{UBB}^{+1}$-triggered cytotoxicity as compared to WT strain (Figure 5B), increasing the lysine concentrations did not promote cytotoxicity in the $\Delta / y s 1$ strain (Figure $5 \mathrm{G}$ ). Thus, in contrast to arginine/ornithine the cellular lysine level appears to be negligible in accelerating $\mathrm{UBB}^{+1}$-triggered cell death. 
A

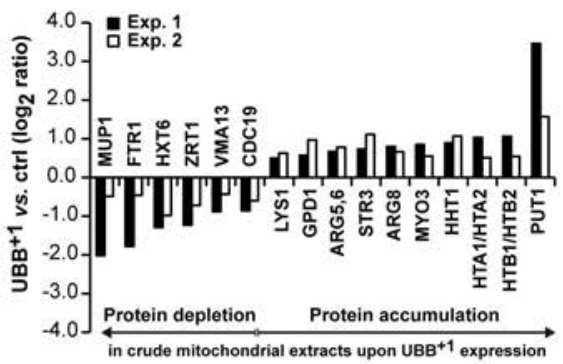

B

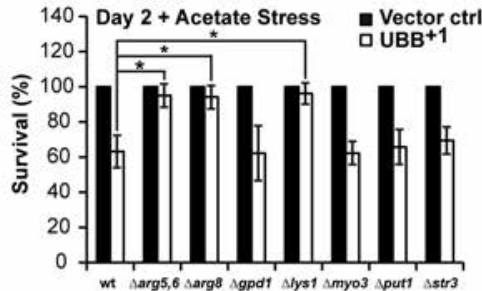

D

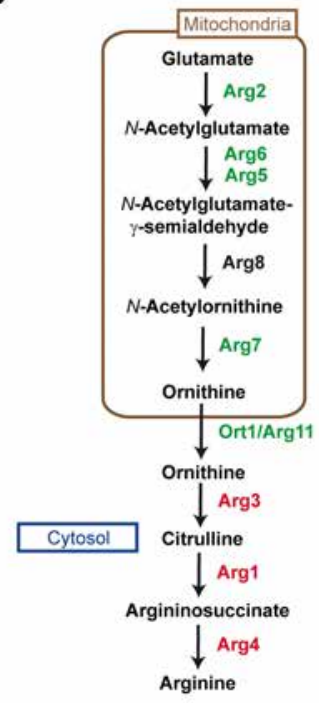

C

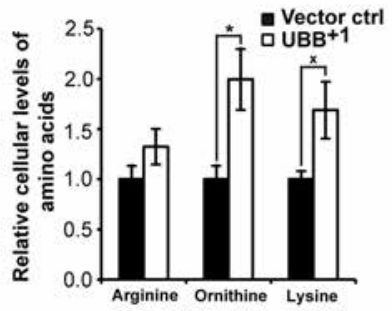

$\mathbf{F}$

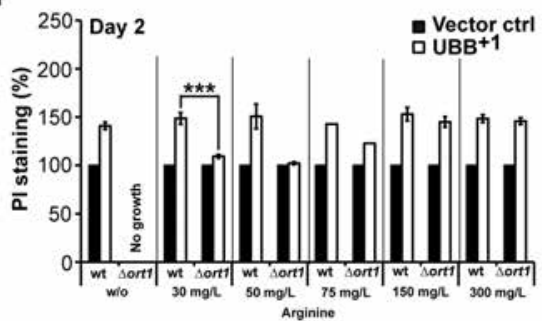

E

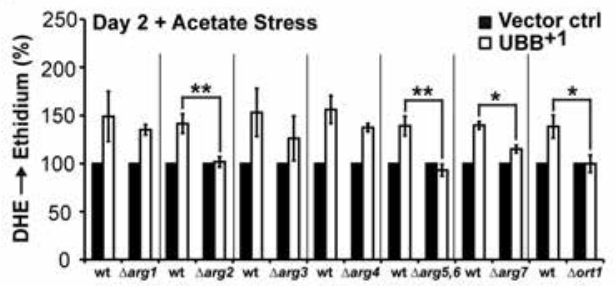

G

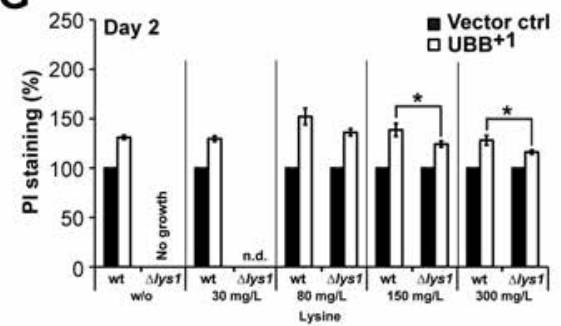

Figure 5: Perturbation of basic amino acid synthesis upon $U B B^{+1}$ expression. A) Protein alterations in crude mitochondria were quantified by SILAC in two independent experiments. Changes were shown which were significant in both experiments. $B) \cup B B^{+1}$-triggered cytotoxicity in strains deleted from genes encoding proteins accumulating in crude mitochondria upon $U_{B B}^{+1}$ expression. Clonogenicity was determined 2 days after inducing expression followed by acetate treatment. The CFUs obtained using cells carrying vector controls were set to $100 \%$ in every experiment. C) Basic 
amino acids were isolated from cultures expressing $U B B^{+1}$ or vector controls, respectively. The mean values of amino acids from cells carrying vector controls were set to 1.0 for every amino acid. D) Arginine and ornithine biosynthetic pathway in S. cerevisiae. Green: Deletion of genes encoding these enzymes does significantly prevent (green), and does not prevent (red) from UBB ${ }^{+1}$-triggered oxidative stress, respectively (see (E) and Figure S5B); Black: no data. E) Oxidative stress in strains with disrupted arginine/ornithine biosynthesis was measured 2 days after inducing $U_{B B^{+1}}$ expression followed by acetate treatment. The oxidative stress levels obtained using cells carrying vector controls were set to $100 \%$ in every experiment. F) Cell death in strains with disrupted arginine/ornithine biosynthesis and increased levels of arginine in the growth media was measured 2 days after inducing $U_{B B}^{+1}$ expression. The proportion of dead cells carrying vector controls was set to $100 \%$ in every experiment. G) Cell death in strains with disrupted lysine biosynthesis and increased levels of lysine in the growth media was measured 2 days after inducing $\mathrm{UBB}^{+1}$ expression. The proportion of dead cells carrying vector controls was set to $100 \%$ in every experiment. Data: percent change values $(B, E-G)$, and mean values $(C)$, respectively. Error bars: standard error. $p$ values: ${ }^{x} p<0.1,{ }^{*} p<0.05,{ }^{* *} p<0.01,{ }^{* * *} p<0.001$. See Tables S1, S2, S4, and Figure S5.

\subsection{Cdc48/Vms1-stimulated mitochondrial UPS protects from UBB ${ }^{+1}$ triggered cytotoxicity}

The aforementioned data incriminate mitochondria and the UPS in the execution of UBB ${ }^{+1}$ triggered cytotoxicity, notably because of the protective impact of the removal of mitochondrial enzymes involved in basic amino acid synthesis and the overexpression of the transcriptional UPS activator Rpn4. Among the known Rpn4 targets are the conserved AAA-ATPase Cdc48 and its cofactor Npl4 (Bosis et al., 2010). Cdc48 and Npl4 are involved in the UPS, and determined by their cofactor Vms1, regulate mitochondrion-associated protein degradation (Heo et al., 2010). Driven by these premises, we evaluated the involvement of the Cdc48/Vms1/Npl4-dependent UPS pathway to UBB $^{+1}$-triggered cytotoxicity. For this, we measured $\mathrm{UBB}^{+1}$-triggered cytotoxicity in normal and acetatestressed conditions in strains expressing increased levels of WT Cdc48 or the pro-apoptotic Cdc48-S565G variant (Madeo et al., 1997), which is characterized by decreased Vms1 binding and mitochondrion-associated degradation (Heo et al., 2010). We also determined the cytotoxicity of $\mathrm{UBB}^{+1}$ in strains depleted from the Cdc48 cofactors Npl4 and Vms1, or overexpressing Vms1. UBB ${ }^{+1}$-triggered cytotoxicity was markedly attenuated in cultures 
expressing increased levels of WT Cdc48, as compared to cells expressing Cdc48-S565G or controls with endogenous Cdc48 only (Figures 6A, S6A). UBB ${ }^{+1}$-triggered cytotoxicity was significantly increased in cultures depleted from Npl4 under non-stressed conditions (Figures 6B, S6B). Depletion of Vms1 resulted in a marked elevation in cytotoxicity upon stress (Figures 6C, S6C), while overexpression of Vms1 significantly protected against $\mathrm{UBB}^{+1}$ upon acetate stress, as measured by the clonogenic approach (Figures 6D, S6D). High levels of $\mathrm{Vms} 1$ also protected from cell death and oxidative stress induced by UBB+1 expression (Figure 6E-F). Notably, high amounts of Cdc48 and Cdc48-S565G resulted in markedly decreased steady-state levels of $\mathrm{UBB}^{+1}$ (Figure S6E-F), whereas neither the deletion of VMS1, nor its overexpression had an effect on the cellular $\mathrm{UBB}^{+1}$ amounts (Figure S6G-J). These data point to a protective role of Vms1, which is independent from $\mathrm{UBB}^{+1}$ degradation, potentially by improving the quality control at mitochondria. In contrast, the beneficial role of high amounts of Cdc48 could be due to both increased Vms1independent $\mathrm{UBB}^{+1}$ degradation, and improved Vms1-dependent mitochondrial quality control.

In order to address whether elevated Vms1 levels prevent from $\mathrm{UBB}^{+1}$-triggered mitochondrial impairment, we measured the cellular oxygen consumption, the mitochondrial membrane potential, and the cellular ATP levels in cells expressing $\mathrm{UBB}^{+1}$ upon endogenous or elevated amounts of Vms1 (Figure 6G-I). Whereas the cellular oxygen consumption, and the mitochondrial membrane potential were significantly decreased by day 3, and days 2 and 3, respectively, cellular ATP levels were significantly increased by day 2 upon high amounts of Vms1. In other words, high amounts of Vms1 reverted the mitochondrial damage induced by high levels of $\mathrm{UBB}^{+1}$ (see Figure $4 \mathrm{C}-\mathrm{E}$ ).

In a next step, we used SILAC technology to comparatively assess alterations of the mitochondrial proteome between $\mathrm{UBB}^{+1}$-expressing cells with endogenous and high levels of Vms1 (Figure 6J, Table S3). We observed that among the 16 proteins whose abundance levels were altered by $\mathrm{UBB}^{+1}$ as compared with the vector control (Figure 5A, Table S2), 10 were no more altered upon expression of both $\mathrm{UBB}^{+1}$ and $\mathrm{Vms} 1$ (Figure $6 \mathrm{~J}$, blue-labelled proteins). Among these 10 proteins, which were particularly stringently associated with the cytopathic activity of $\mathrm{UBB}^{+1}$, the basic amino acid synthesis enzymes Arg5,6, Arg8, and 
Lys1 were significantly decreased in $\mathrm{UBB}^{+1}$-expressing cells upon high levels of Vms1, as compared to endogenous Vms1 levels. Consistently, Vms1 overexpression blunted the $\mathrm{UBB}^{+1}$-mediated increase in the steady-state levels of arginine, ornithine, and lysine (Figure 6K, see Figure 5C). These data point to a pivotal role of the Vms1-dependent mitochondrial UPS activity in avoiding the $\mathrm{UBB}^{+1}$-triggered lethal overproduction of basic amino acids. 
A
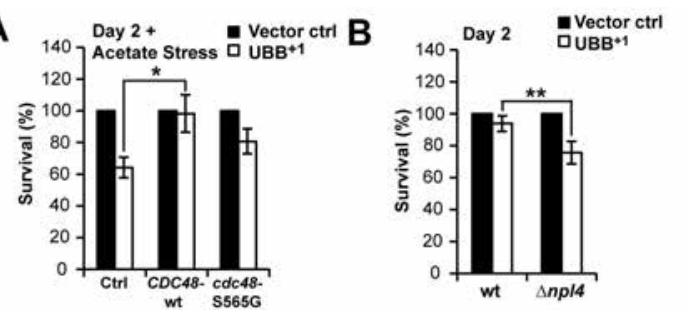

D ฐ

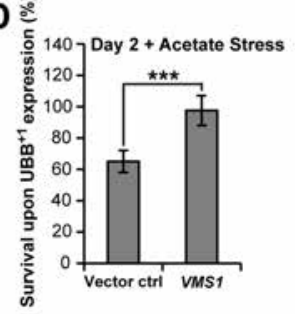

G

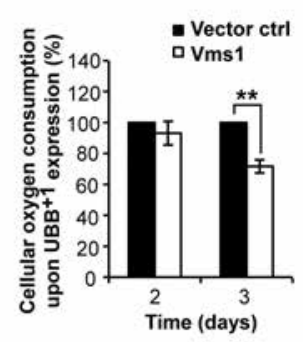

$\mathbf{J}$

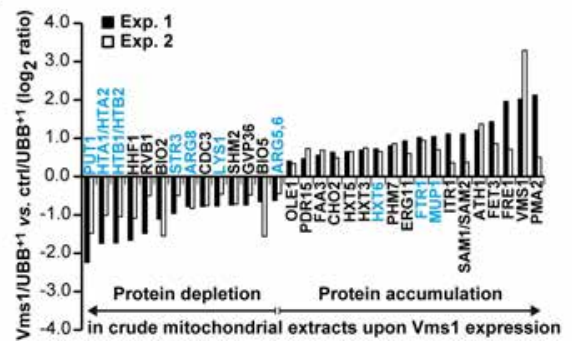

H

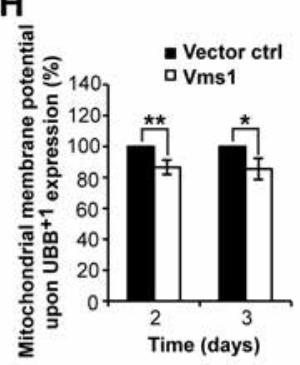

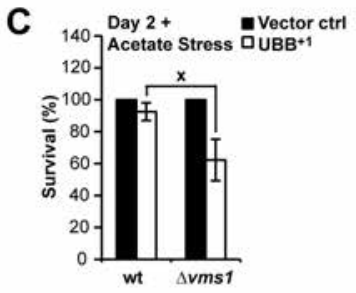

$\mathbf{F}$

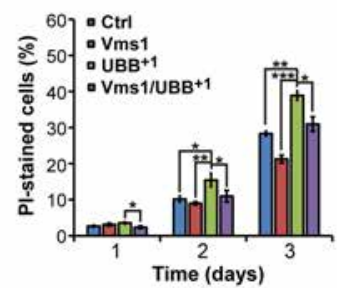

I

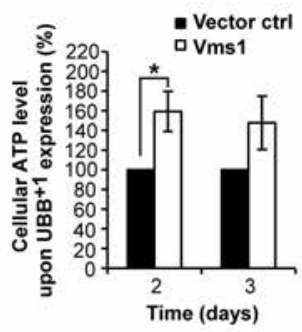

K

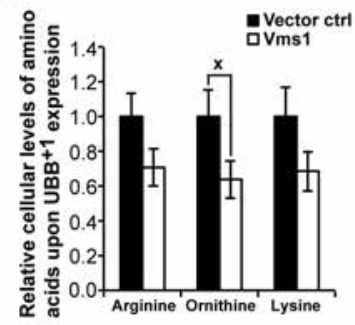

Figure 6: Role of Cdc48/Np/4/Vms1 complex in UBB ${ }^{+1}$-triggered cytotoxicity. A-C) UBB ${ }^{+1}$ was expressed in strains with elevated levels of Cdc48 or Cdc48-S565G (A), and strains deleted for NPL4 $(B)$ and VMS1 (C). Clonogenicity was determined 2 days after inducing expression before (B) and after acetate stress $(A, C)$. The CFUs obtained using cells carrying vector controls were set to $100 \%$ in every experiment. D) Clonogenicity of $U B B^{+1}$-expressing cultures in strains with endogenous (vector control) and elevated levels of Vms1 (Vms1), respectively. Clonogenicity was determined 2 days after inducing expression followed by acetate treatment. The CFUs obtained using cells with endogenous and elevated levels of Vms1, respectively, but lacking $U \mathrm{BB}^{+1}$, were set to $100 \%$ in every 
experiment (not shown). E-F) Cell death and oxidative stress was measured 1, 2, and 3 days after inducing expression of $U B B^{+1}$ and/or Vms1. G-I) $U B B^{+1}$ was expressed in WT strain with endogenous (vector ctrl) and increased levels of Vms1. Cellular oxygen consumption (G), mitochondrial membrane potential $(H)$, and cellular ATP levels (I), were determined 2 and 3 days after inducing expression. The oxygen consumption (G), mitochondrial membrane potential (H), and ATP levels (I) measured using cells with endogenous Vms1 were set to $100 \%$ in every experiment. J) Protein alterations in crude mitochondria upon Vms1 expression. Mitochondria were isolated from cultures expressing $U_{B B^{+1}}$ in cells with increased or endogenous levels of Vms1, respectively. Protein alterations were quantified by SILAC in two independent experiments. Changes were shown which were significant in both experiments. Blue-labelled proteins are inversely regulated as compared to Figure 5A. K) Cellular levels of basic amino acids upon Vms1 expression. Basic amino acids were isolated from cultures expressing $U \mathrm{UB}^{+1}$ in cells with endogenous (vector control) or increased levels of Vms1 (Vms1), respectively. The mean values of amino acids from cells with endogenous Vms1 levels were set to 1.0 for every amino acid. Data: percent change values ( $A-D, G-I)$, and mean values $(E, F, K)$, respectively. Error bars: standard error. $p$-values: $x p<0.1,{ }^{*} p<0.05,{ }^{* *} p<0.01,{ }^{* * *} p<$ 0.001. See Tables S1, S3, S4, and Figure S6.

\subsection{VMS1 co-exists with tau and $\mathrm{UBB}^{+1}$ in hippocampal neurons from AD patients}

The hippocampus is severely affected during AD progression. Pathological hallmarks include intracellular neurofibrillary tangles comprising aberrant forms of the microtubuleassociated protein tau, $\mathrm{UBB}^{+1}$, and the mitochondrial outer membrane voltage-dependent anion channel 1 (VDAC1) (van Leeuwen et al., 1998;Reddy, 2013). Immunohistochemistry revealed expression of VMS1, the human homolog of yeast Vms1, in pyramidal cells within the hippocampi from AD patients and aged non-demented controls (Figure 7A, all arrows; Tables S5-S6). VMS1-stained structures reminiscent of tau pathology, including tangle-like (yellow arrows) and neuropil thread-like (NP) structures (blue arrows), as well as other cellular staining patterns (green arrows) were observed in samples from AD patients, and aged non-demented controls with tau pathology. We also observed these tangle-like and thread-like staining patterns when analyzing the sections for aberrant tau, $\mathrm{UBB}^{+1}$, and VDAC1 (Figure S7). Immunohistochemistry of consecutive paraffin sections from the hippocampi of $A D$ patients (Figure 7B) confirmed the identification of pyramidal cells with 
intracellular tangle-like structures, which co-stained for aberrant tau and VMS1 (Figure 7C, violet arrows), and for UBB ${ }^{+1}$, VMS1 and VDAC1 (Figure 7D, orange arrows). These data suggest that VMS1 is a component of NFTs comprising aberrant tau, UBB ${ }^{+1}$ and VDAC1, underscoring a potential role of the Cdc48/VMS1 complex in UBB ${ }^{+1}$-mediated $A D$ progression. 
A
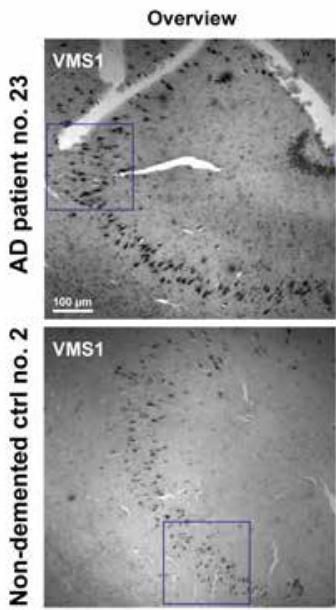

Tangle-like structures

4 Neuropil thread-like structures

4 Other stained structures

C

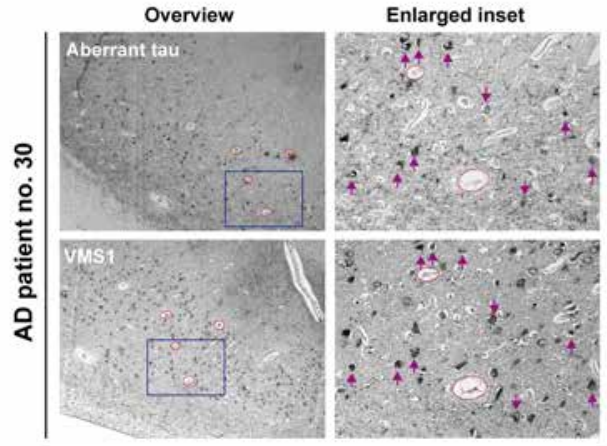

4 Structures co-stained for aberrant tau and VMS1 Blood vessels for orientation
B

Principle of the staining of the consecutive sections shown in $7 C$ and $7 D$

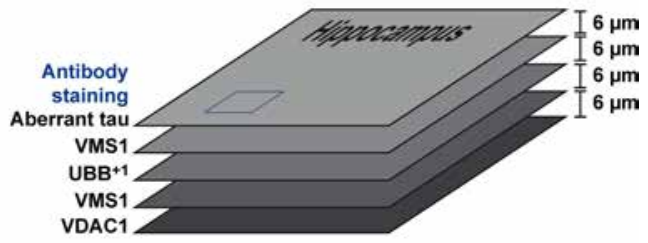

D

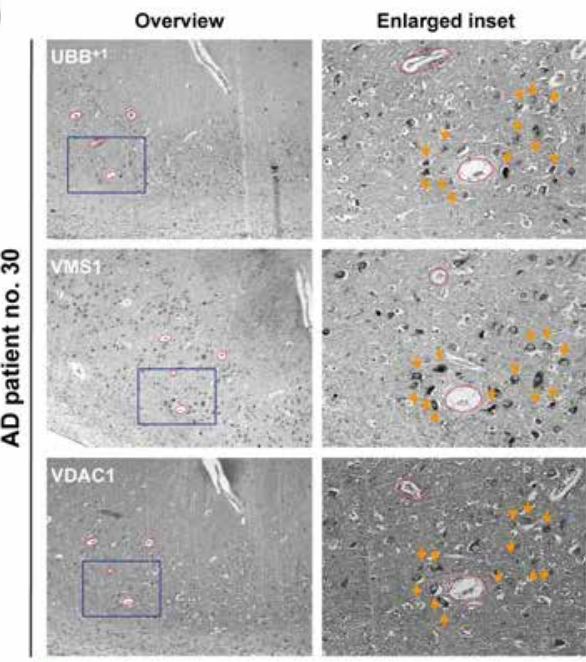

4 Structures co-stained for UBB ${ }^{+1}$, VMS1 and VDAC1 Blood vessels for orientation

Figure 7: VMS1 co-existence with aberrant tau, UBB ${ }^{+1}$ or VDAC1 in hippocampi of AD patients. A) VMS1 staining in AD patient and non-demented control. B) Principle of the staining of consecutive sections from the hippocampus of an $A D$ patient shown in (C) and (D). C) Co-existence of aberrant tau (MC1) and VMS1. D) Co-existence of UBB ${ }^{+1}$ (Ubi2a), VMS1, and VDAC1. See Figure S7, and Tables S5-S6. 


\section{Discussion}

We established a yeast model for dissecting cell death mechanisms triggered by $\mathrm{UBB}^{+1}$, in which mitochondria play a pivotal role in the execution of cell death (see Supplemental Discussion). $\mathrm{UBB}^{+1}$ triggers neuronal apoptosis accompanied by reduced mitochondrial movement (Tan et al., 2007), and mitochondrial impairment likely contributes to $A D$ (Rodolfo et al., 2010). Thus, our yeast model corroborates features of cell death-relevant mitochondrion dysfunctions found in $A D$ neurons.

Yeast strains expressing $\mathrm{UBB}^{+1}$ accumulated the basic amino acids arginine, ornithine, and lysine. Deletion of mitochondrion-associated enzymes involved in their synthesis abolished $\mathrm{UBB}^{+1}$-triggered cell killing, which could be recovered by increasing concentrations of arginine in the growth media. The accumulation of basic amino acids may trigger mitochondrial damage and cell death in mammalian cells and in yeast (Almeida et al., 2007; Biczo et al., 2011). For instance, increased production of nitric oxide from arginine executes yeast apoptosis (Almeida et al., 2007); and increased levels of polyamines, which are produced from ornithine, may impair neuronal ion channel activities (Inoue et al., 2013). The levels of arginine, ornithine and/or their polyamine products were altered in aged human brains and in brains from AD patients (Rushaidhi et al., 2012; Inoue et al., 2013; Liu et al., 2014). The results of these studies are controversial, and it remains not yet clear whether the observed alterations are cause or consequence of AD. Despite of that, our data suggest that perturbed basic amino acid synthesis is a decisive event triggering mitochondrion-dependent cell death upon $\mathrm{UBB}^{+1}$ accumulation in yeast. Further studies aiming at analyzing the role of arginine/ornithine metabolism during aging or $A D$ progression should consider a potential pivotal contribution of UPS and mitochondrial dysfunctions.

We observed that $\mathrm{UBB}^{+1}$ accumulation impaired the UPS, and that the UPS activity in turn determined $\mathrm{UBB}^{+1}$ cytotoxicity. Yeast cultures that were depleted from ubiquitin ( $\triangle u b i 4$ ) were highly vulnerable to $\mathrm{UBB}^{+1}$. In contrast, yeast cultures in which the UPS was stimulated by the transcriptional activator Rpn4 were insensitive to $\mathrm{UBB}^{+1}$, but not in cells lacking the ubiquitin gene UBI4. Extended ubiquitin variants have been proposed to be specific inhibitors of the deubiquitinase Ubp6 in yeast (Krutauz et al., 2014). Since the 
$\mathrm{UBB}^{+1}$-triggered cytotoxicity was unaltered in a strain deleted for UBP6 as compared to WT strain, our data suggest that the lethal effect of the extended ubiquitin $U_{B B}{ }^{+1}$ does not essentially depend on Ubp6. It is tempting to speculate, that the ratio of mutant $\left(\mathrm{UBB}^{+1}\right)$ to WT ubiquitin determines $\mathrm{UBB}^{+1}$-triggered cytotoxicity with $\mathrm{UBB}^{+1}$ as a competitive inhibitor of WT ubiquitin, affecting numerous ubiquitin-regulated cellular processes.

We established that elevated amounts of Cdc48 or its cofactor Vms1 conferred tolerance against $\mathrm{UBB}^{+1}$ expression. More specifically, Vms1 overexpression relieved the UBB ${ }^{+1}$ triggered mitochondrial damage and accumulation of the basic amino acids arginine, ornithine, and lysine. The Cdc48/Vms1 complex enables the degradation of mitochondrionassociated proteins (Heo et al., 2010). Whereas under normal conditions, this complex is predominantly cytosolic, Vms1 recruits Cdc48 to the mitochondrial outer membrane upon stress, presumably with the scope of improving the local quality of proteins. Our data suggest that Cdc48/Vms1-mediated processes can prevent the $\mathrm{UBB}^{+1}$-triggered lethal derangement of mitochondria. In one possible scenario, Cdc48/Vms1 might remove protein junk from the mitochondrial outer membrane. Alternatively, Cdc48/Vms1 might specifically prevent the accumulation of arginine, ornithine, and lysine, through regulation of the turnover of the enzymes Arg5,6, Arg8, and Lys1, which are pivotal for their synthesis. Whereas the activity of the cytoplasmic enzyme Lys1 could be regulated by its degradation, the activities of the mitochondrion-associated Arg5,6 and Arg8 could be controlled by preventing their import into mitochondria via ubiquitination and proteasomal degradation. Lys1, Arg5, 6 and Arg8 are known targets for ubiquitination (Xu et al., 2009), and the UPS regulates the import of mitochondrial intermembrane space proteins (Bragoszewski et al., 2013; Harbauer et al., 2014). It is tempting to speculate for a UPS-dependent regulation of the import of the mitochondrial matrix proteins Arg5, 6 and Arg8. Further studies are needed to address the influence of UPS (dys)function on the turnover of these and other mitochondrial proteins. This is of importance, because recent studies demonstrated that UPS dysfunction can lead to mitochondrial dysfunction and vice versa (Livnat-Levanon et al., 2014;Maharjan et al., 2014; Segref et al., 2014); and our data revealed the unexpected link between $\mathrm{UBB}^{+1}$-triggered UPS dysfunction and the accumulation of functional enzymes in the mitochondrial matrix leading to potentially cytotoxic accumulation of basic amino acids. 
Human VMS1 and mitochondrial VDAC1 co-existed with UBB ${ }^{+1}$ in neurofibrillary tangles of $A D$ patients and aged non-demented controls with tau pathology. UBB ${ }^{+1}$ accumulates and the number of neurofibrillary tangles and damaged mitochondria markedly increase during AD progression (Dennissen et al., 2010;Rodolfo et al., 2010). We propose that VMS1dependent mitochondrial quality control might retard the AD-associated neuronal dysfunction, which is elicited by the accumulation of both aberrant tau and UBB ${ }^{+1}$. 


\section{References}

Almeida, B., Büttner, S., Ohlmeier, S., Silva, A., Mesquita, A., Sampaio-Marques, B., Osorio, N.S., Kollau, A., Mayer, B., Leao, C., Laranjinha, J., Rodrigues, F., Madeo, F., and Ludovico, P. (2007). NO-mediated apoptosis in yeast. J Cell Sci 120, 3279-3288.

Biczo, G., Hegyi, P., Dosa, S., Shalbuyeva, N., Berczi, S., Sinervirta, R., Hracsko, Z., Siska, A., Kukor, Z., Jarmay, K., Venglovecz, V., Varga, I.S., Ivanyi, B., Alhonen, L., Wittmann, T., Gukovskaya, A., Takacs, T., and Rakonczay, Z., Jr. (2011). The crucial role of early mitochondrial injury in L-lysine-induced acute pancreatitis. Antioxid Redox Signal 15, 26692681.

Bosis, E., Salomon, D., Ohayon, O., Sivan, G., Bar-Nun, S., and Rabinovich, E. (2010). Ssz1 restores endoplasmic reticulum-associated protein degradation in cells expressing defective colc48ufd1-npl4 complex by upregulating cdc48. Genetics 184, 695-706.

Bragoszewski, P., Gornicka, A., Sztolsztener, M.E., and Chacinska, A. (2013). The ubiquitinproteasome system regulates mitochondrial intermembrane space proteins. Mol Cell Biol $33,2136-2148$

Braun, R.J. (2012). Mitochondrion-mediated cell death: dissecting yeast apoptosis for a better understanding of neurodegeneration. Front Oncol 2, 182.

Braun, R.J., Büttner, S., Ring, J., Kroemer, G., and Madeo, F. (2010). Nervous yeast: modeling neurotoxic cell death. Trends Biochem Sci 35, 135-144.

Büttner, S., Habernig, L., Broeskamp, F., Ruli, D., Vogtle, F.N., Vlachos, M., Macchi, F., Kuttner, V., Carmona-Gutierrez, D., Eisenberg, T., Ring, J., Markaki, M., Taskin, A.A., Benke, S., Ruckenstuhl, C., Braun, R., Van Den Haute, C., Bammens, T., Van Der Perren, A., Fröhlich, K.-U., Winderickx, J., Kroemer, G., Baekelandt, V., Tavernarakis, N., Kovacs, G.G., Dengjel, J., Meisinger, C., Sigrist, S.J., and Madeo, F. (2013). Endonuclease G mediates alphasynuclein cytotoxicity during Parkinson's disease. EMBO J 32, 3041-3054.

Carmona-Gutierrez, D., Ruckenstuhl, C., Bauer, M.A., Eisenberg, T., Büttner, S., and Madeo, F. (2010). Cell death in yeast: growing applications of a dying buddy. Cell Death Differ 17, 733734.

De Vrij, F.M., Sluijs, J.A., Gregori, L., Fischer, D.F., Hermens, W.T., Goldgaber, D., Verhaagen, J., Van Leeuwen, F.W., and Hol, E.M. (2001). Mutant ubiquitin expressed in Alzheimer's disease causes neuronal death. FASEB J 15, 2680-2688.

Debattisti, V., and Scorrano, L. (2013). D. melanogaster, mitochondria and neurodegeneration: small model organism, big discoveries. Mol Cell Neurosci 55, 77-86.

Dennissen, F.J., Kholod, N., Hermes, D.J., Kemmerling, N., Steinbusch, H.W., Dantuma, N.P., and Van Leeuwen, F.W. (2011). Mutant ubiquitin $\left(\mathrm{UBB}^{+1}\right)$ associated with neurodegenerative disorders is hydrolyzed by ubiquitin C-terminal hydrolase L3 (UCH-L3). FEBS Lett 585, 2568-2574.

Dennissen, F.J., Kholod, N., Steinbusch, H.W., and Van Leeuwen, F.W. (2010). Misframed proteins and neurodegeneration: a novel view on Alzheimer's and Parkinson's diseases. Neurodegener Dis 7, 76-79.

Diaz, F., Enriquez, J.A., and Moraes, C.T. (2012). Cells lacking Rieske iron-sulfur protein have a reactive oxygen species-associated decrease in respiratory complexes I and IV. Mol Cell Biol 32, 415-429. 
Eisenberg, T., Büttner, S., Kroemer, G., and Madeo, F. (2007). The mitochondrial pathway in yeast apoptosis. Apoptosis 12, 1011-1023.

Finley, D., Ozkaynak, E., and Varshavsky, A. (1987). The yeast polyubiquitin gene is essential for resistance to high temperatures, starvation, and other stresses. Cell 48, 1035-1046.

Fischer, D.F., Van Dijk, R., Van Tijn, P., Hobo, B., Verhage, M.C., Van Der Schors, R.C., Li, K.W., Van Minnen, J., Hol, E.M., and Van Leeuwen, F.W. (2009). Long-term proteasome dysfunction in the mouse brain by expression of aberrant ubiquitin. Neurobiol Aging 30, 847863.

Harbauer, A.B., Zahedi, R.P., Sickmann, A., Pfanner, N., and Meisinger, C. (2014). The protein import machinery of mitochondria-a regulatory hub in metabolism, stress, and disease. Cell Metab 19, 357-372.

Hauptmann, P., and Lehle, L. (2008). Kex1 protease is involved in yeast cell death induced by defective N-glycosylation, acetic acid, and chronological aging. J Biol Chem 283, 1915119163.

Heinemeyer, W., Gruhler, A., Mohrle, V., Mahe, Y., and Wolf, D.H. (1993). PRE2, highly homologous to the human major histocompatibility complex-linked RING10 gene, codes for a yeast proteasome subunit necessary for chrymotryptic activity and degradation of ubiquitinated proteins. J Biol Chem 268, 5115-5120.

Heo, J.M., Livnat-Levanon, N., Taylor, E.B., Jones, K.T., Dephoure, N., Ring, J., Xie, J., Brodsky, J.L., Madeo, F., Gygi, S.P., Ashrafi, K., Glickman, M.H., and Rutter, J. (2010). A stressresponsive system for mitochondrial protein degradation. Mol Cell 40, 465-480.

Inoue, K., Tsutsui, H., Akatsu, H., Hashizume, Y., Matsukawa, N., Yamamoto, T., and Toyo'oka, T. (2013). Metabolic profiling of Alzheimer's disease brains. Sci Rep 3, 2364.

Kruegel, U., Robison, B., Dange, T., Kahlert, G., Delaney, J.R., Kotireddy, S., Tsuchiya, M., Tsuchiyama, S., Murakami, C.J., Schleit, J., Sutphin, G., Carr, D., Tar, K., Dittmar, G., Kaeberlein, M., Kennedy, B.K., and Schmidt, M. (2011). Elevated proteasome capacity extends replicative lifespan in Saccharomyces cerevisiae. PLoS Genet 7, e1002253.

Krutauz, D., Reis, N., Nakasone, M.A., Siman, P., Zhang, D., Kirkpatrick, D.S., Gygi, S.P., Brik, A., Fushman, D., and Glickman, M.H. (2014). Extended ubiquitin species are protein-based DUB inhibitors. Nat Chem Biol 10, 664-670.

Lindsten, K., De Vrij, F.M., Verhoef, L.G., Fischer, D.F., Van Leeuwen, F.W., Hol, E.M., Masucci, M.G., and Dantuma, N.P. (2002). Mutant ubiquitin found in neurodegenerative disorders is a ubiquitin fusion degradation substrate that blocks proteasomal degradation. $\mathrm{J}$ Cell Biol $157,417-427$.

Liu, P., Fleete, M.S., Jing, Y., Collie, N.D., Curtis, M.A., Waldvogel, H.J., Faull, R.L., Abraham, W.C., and Zhang, H. (2014). Altered arginine metabolism in Alzheimer's disease brains. Neurobiol Aging 35, 1992-2003.

Livnat-Levanon, N., Kevei, E., Kleifeld, O., Krutauz, D., Segref, A., Rinaldi, T., Erpapazoglou, Z., Cohen, M., Reis, N., Hoppe, T., and Glickman, M.H. (2014). Reversible 26S proteasome disassembly upon mitochondrial stress. Cell Rep 7, 1371-1380.

Ljungdahl, P.O., and Daignan-Fornier, B. (2012). Regulation of amino acid, nucleotide, and phosphate metabolism in Saccharomyces cerevisiae. Genetics 190, 885-929. 
Madeo, F., Fröhlich, E., and Fröhlich, K.U. (1997). A yeast mutant showing diagnostic markers of early and late apoptosis. J Cell Biol 139, 729-734.

Maharjan, S., Oku, M., Tsuda, M., Hoseki, J., and Sakai, Y. (2014). Mitochondrial impairment triggers cytosolic oxidative stress and cell death following proteasome inhibition. Sci Rep 4, 5896.

Mannhaupt, G., Schnall, R., Karpov, V., Vetter, I., and Feldmann, H. (1999). Rpn4p acts as a transcription factor by binding to PACE, a nonamer box found upstream of 26S proteasomal and other genes in yeast. FEBS Lett 450, 27-34.

Reddy, P.H. (2013). Is the mitochondrial outermembrane protein VDAC1 therapeutic target for Alzheimer's disease? Biochim Biophys Acta 1832, 67-75.

Rodolfo, C., Ciccosanti, F., Giacomo, G.D., Piacentini, M., and Fimia, G.M. (2010). Proteomic analysis of mitochondrial dysfunction in neurodegenerative diseases. Expert Rev Proteomics 7, 519-542.

Rushaidhi, M., Jing, Y., Kennard, J.T., Collie, N.D., Williams, J.M., Zhang, H., and Liu, P. (2012). Aging affects L-arginine and its metabolites in memory-associated brain structures at the tissue and synaptoneurosome levels. Neuroscience 209, 21-31.

Segref, A., Kevei, E., Pokrzywa, W., Schmeisser, K., Mansfeld, J., Livnat-Levanon, N., Ensenauer, R., Glickman, M.H., Ristow, M., and Hoppe, T. (2014). Pathogenesis of human mitochondrial diseases is modulated by reduced activity of the ubiquitin/proteasome system. Cell Metab 19, 642-652.

Tan, Z., Sun, X., Hou, F.S., Oh, H.W., Hilgenberg, L.G., Hol, E.M., Van Leeuwen, F.W., Smith, M.A., O'dowd, D.K., and Schreiber, S.S. (2007). Mutant ubiquitin found in Alzheimer's disease causes neuritic beading of mitochondria in association with neuronal degeneration. Cell Death Differ 14, 1721-1732.

Tank, E.M., and True, H.L. (2009). Disease-associated mutant ubiquitin causes proteasomal impairment and enhances the toxicity of protein aggregates. PLoS Genet 5, e1000382.

Van Leeuwen, F.W., De Kleijn, D.P., Van Den Hurk, H.H., Neubauer, A., Sonnemans, M.A., Sluijs, J.A., Koycu, S., Ramdjielal, R.D., Salehi, A., Martens, G.J., Grosveld, F.G., Peter, J., Burbach, H., and Hol, E.M. (1998). Frameshift mutants of beta amyloid precursor protein and ubiquitin-B in Alzheimer's and Down patients. Science 279, 242-247.

Van Tijn, P., De Vrij, F.M., Schuurman, K.G., Dantuma, N.P., Fischer, D.F., Van Leeuwen, F.W., and Hol, E.M. (2007). Dose-dependent inhibition of proteasome activity by a mutant ubiquitin associated with neurodegenerative disease. J Cell Sci 120, 1615-1623.

Van Tijn, P., Verhage, M.C., Hobo, B., Van Leeuwen, F.W., and Fischer, D.F. (2010). Low levels of mutant ubiquitin are degraded by the proteasome in vivo. J Neurosci Res 88, 2325-2337.

Xu, P., Duong, D.M., Seyfried, N.T., Cheng, D., Xie, Y., Robert, J., Rush, J., Hochstrasser, M., Finley, D., and Peng, J. (2009). Quantitative proteomics reveals the function of unconventional ubiquitin chains in proteasomal degradation. Cell 137, 133-145. 


\section{CHAPTER 5}

Mutant ubiquitin decreases amyloid $\beta$ plaque formation in a transgenic mouse model of Alzheimer's disease.

P. van Tijn*, F.J.A. Dennissen*, R.J. Gentier, B. Hobo, D.J.H.P. Hermes, H.W.M. Steinbusch, F.W. Van Leeuwen and D.F. Fischer. Neurochemistry International.2012;61(5):739-748

${ }^{*}$ contributed equally

Supplementary material available at:

http://dx.doi.org/10.1016/j.neuint.2012. 07.007. 


\section{Abstract}

The mutant ubiquitin $\mathrm{UBB}^{+1}$ is a substrate as well as an inhibitor of the ubiquitin-proteasome system (UPS) and accumulates in the neuropathological hallmarks of Alzheimer's disease (AD). A role for the UPS has been suggested in the generation of amyloid $\beta(A \beta)$ plaques in $\mathrm{AD}$. To investigate the effect of $\mathrm{UBB}^{+1}$ expression on amyloid pathology in vivo, we crossed $\mathrm{UBB}^{+1}$ transgenic mice with a transgenic line expressing $\mathrm{AD}$-associated mutant amyloid precursor protein (APPSwe) and mutant presenilin 1 (PS1dE9), resulting in APPPS1/UBB ${ }^{+1}$ triple transgenic mice. In these mice, we determined the $A \beta$ levels at 3,6 , 9 and 11 months of age. Surprisingly, we found a significant decrease in $A \beta$ deposition in amyloid plaques and levels of soluble $A \beta_{42}$ in APPPS1/UBB ${ }^{+1}$ transgenic mice compared to APPPS1 mice at 6 months of age, without alterations in $\mathrm{UBB}^{+1}$ protein levels or proteasomal chymotrypsin activity. These lowering effects of $U_{B B}^{+1}$ on $A \beta$ deposition were transient, as this relative decrease in plaque load was not significant in APPPS1/UBB ${ }^{+1}$ mice at 9 and 11 months of age. We also show that APPPS1/UBB ${ }^{+1}$ mice exhibit astrogliosis, indicating that they may not be improved functionally compared to APPPS1 mice despite the $A \beta$ reduction. The molecular mechanism underlying this decrease in $A \beta$ deposition in APPPS1/UBB ${ }^{+1}$ mice is more complex than previously assumed because $\mathrm{UBB}^{+1}$ is also ubiquitinated at $\mathrm{K} 63$ opening the possibility of additional effects of $\mathrm{UBB}^{+1}$ (e.g. , kinase activation). 


\section{Introduction}

One of the main neuropathological hallmarks of Alzheimer's disease (AD) is the deposition of amyloid $\beta(A \beta)$ in extracellular plaques. The $A \beta$ peptide is generated via $\beta$ - and $\gamma$ secretase mediated proteolytic cleavage of the amyloid precursor protein (APP) (Selkoe, 2001). According to the "amyloid hypothesis", accumulation of $A \beta$, especially aggregateprone $A \beta_{42}$, is the primary event in AD pathogenesis (Hardy and Selkoe, 2002; Hardy, 2009). Increasing evidence implicates impairment of the ubiquitin-proteasome system (UPS), the main intracellular regulated proteolytic pathway, as well as impaired autophagy, in the pathogenesis of AD (Ciechanover and Brundin, 2003;Nixon et al., 2008). In keeping with this, proteasome activity and ubiquitination enzymes are decreased in $A D$ brains (Keller et al., 2000;Lopez Salon et al., 2000). A $\beta$ dose-dependently decreases UPS activity in vitro (Gregori et al., 1995), (Zhao and Yang, 2010) and in vivo in an age-dependent manner, as was shown in an APPSwe transgenic (tg) mouse model of AD in mice older than 15 months of age (Oh et al., 2005;Almeida et al., 2006). However, proteasome inhibition also affects APP processing, variably resulting in increased (Nunan et al., 2001; Flood et al., 2005) or decreased A $\beta$ production (Christie et al., 1999;Kienlen-Campard et al., 2006).

A mutant form of ubiquitin $\left(\mathrm{UBB}^{+1}\right)$ accumulates in the neuropathological hallmarks of both sporadic AD (van Leeuwen et al., 1998) and early-onset familial AD (van Leeuwen et al., 2006). Although $U_{B B}^{+1}$ is ubiquitinated and directed to the proteasome for proteolytic degradation (Lindsten et al., 2002), it has been shown that the C-terminal extension of $\mathrm{UBB}^{+1}$ is 5 amino acids too short to be efficiently degraded (Verhoef et al., 2009). In addition, it has been shown recently that $\mathrm{UBB}^{+1}$ can be C-terminally truncated by the ubiquitin hydrolase UCH-L3 (Dennissen et al., 2011). The crystal structure of $\mathrm{UBB}^{+1}$ gives a structural basis of E2-25K/UBB ${ }^{+1}$ interaction leading to proteasome inhibition and neurotoxicity (Ko et al., 2010). Consequently, abnormal accumulation of $\mathrm{UBB}^{+1}$ may serve as an endogenous marker for UPS dysfunction (Fischer et al., 2003). UBB ${ }^{+1}$ also acts as UPS inhibitor in vitro when expressed at high concentrations (van Tijn et al., 2007). Indeed, in the recently described $\mathrm{UBB}^{+1} \operatorname{tg}$ mouse line 3413 , chymotryptic proteasome activity in the cerebral cortex is decreased to $80 \%$ of normal levels at 8 months of age, accompanied 
by accumulation of ubiquitinated proteins in the cortex and behavioral changes, as shown by a decreased context-dependent memory (Fischer et al., 2009;van Tijn et al., 2011). Free ubiquitin levels however appear not to be affected in this mouse model, suggesting that the observed phenotypes are not caused through depletion of free ubiquitin by UBB ${ }^{+1}$.

In the present study we analysed the effect of $\mathrm{UBB}^{+1}$ expression on $\mathrm{A} \beta$ deposition to further elucidate the contribution of dysfunctional ubiquitin to $A D$ pathogenesis in vivo. We therefore crossed the $\operatorname{tg} \mathrm{UBB}^{+1}$ line 3413 with a tg $A D$ line co-expressing familial AD-linked APP (APPSwedish) and presenilin 1 (PS1dE9) (hereafter referred to as line APPPS1) which shows increased $A \beta_{42}$ production accompanied by dense-core plaque pathology in the brain (Jankowsky et al., 2004). In the resulting APPPS1/UBB ${ }^{+1}$ triple tg mice, we analyzed the effects of $\mathrm{UBB}^{+1}$ on $A D$ related pathology as well as the effects of $A \beta$ deposition on $\mathrm{UBB}^{+1}$ accumulation in vivo.

\section{Materials and Methods}

\subsection{Transgenic mice}

In the present study, we used the $\mathrm{UBB}^{+1} \operatorname{tg}$ mouse line 3413 (Fischer et al., 2009), that expresses human $U B B^{+1} \mathrm{CDNA}$ in neurons under control of the murine CamKlla promoter on a pure C57/BI6 background. The double tg APPPS1 (line 85, APPSwe/PS1dE9), previously described by (Jankowsky et al., 2004), carries a co-integrate of 1) chimeric mouse/human APP695 carrying the Swedish mutation (K594M/N595L) and 2) human PS1 with deletion of exon 9 (Jankowsky et al., 2001), each under control of a mouse prion protein promoter. Line 85 was back-crossed to $\mathrm{C} 57 / \mathrm{Bl} 6$ for at least seven generations and thus can also be assumed to be of pure $\mathrm{C} 57 \mathrm{~B} / / 6$ background. Subsequently heterozygous APPPS1 mice were crossed to heterozygous line 3413 mice to generate triple tg APPPS1/UBB ${ }^{+1}$ mice. The APPPS1, $\mathrm{UBB}^{+1} \mathrm{tg}$ mice and wildtype (WT) littermates were used as controls. Mice were kept in group housing on a 12/12h light-dark cycle with food and water ad libitum in specific pathogen free conditions (Nicklas et al., 2002). All animal experiments were performed conforming to national animal welfare law and under 
guidance of the animal welfare committees of the Royal Netherlands Academy of Arts and Sciences and of Maastricht University.

\subsection{Immunohistochemistry}

Animals were deeply anesthetized with intra-peritoneal sodium pentobarbital and were perfused by intracardially with phosphate-buffered saline (PBS) $\mathrm{pH} \mathrm{7.4,} \mathrm{followed} \mathrm{by} \mathrm{PBS}$ containing 4\% paraformaldehyde. Brains were removed and stored in PBS-4\% paraformaldehyde at $4^{\circ} \mathrm{C}$ until further processing. Brains were subsequently bisected along the midline; the left hemisphere was sectioned in $50 \mu \mathrm{m}$ coronal sections on a Vibratome (Leica VT1000S). For sagittal sections brains were embedded in gelatine and cut in 50 $\mathrm{mm}$ sections similarly. To detect and quantify $\mathrm{UBB}^{+1}$ protein levels, every tenth coronal section was immunohistochemically stained overnight with rabbit polyclonal anti-UBB ${ }^{+1}$ antibody (Ubi3; bleeding date 05/08/97, 1:1,000 (Fischer et al., 2003)), as described using the peroxidase-anti-peroxidase method (Sternberger et al., 1970). Staining was visualized with 3,3'-diaminobenzidine (DAB) solution using nickel intensification (0.2\%). An additional series of every tenth section was used to detect and quantify the $A \beta$ plaque load by immunohistochemical staining of sections overnight with mouse monoclonal anti-A $\beta$ antibody 6E10 (1:16,000; Signet 9300-02) with a 30min pretreatment with fresh formic acid solution to permeabilize the tissue. Sections were further processed using the peroxidaseanti-peroxidase method and 3,3'-diaminobenzidine-Ni color reaction. The sagittal sections were stained with mouse monoclonal anti-GFAP antibody GF5 (1:400; Abcam AB 10062) and rabbit polyclonal GFAP (1:2000; DAKO Z0334), incubated with either donkey-antimouse-biotin (1:400; Jackson) or donkey anti-rabbit-biotin (1:400; Jackson) and ABC (1:400; Vector) and similarly stained with 3,3'-diaminobenzidine-Ni color reaction. Stained sections were mounted on glass slides, embedded using Entellan (Merck) and cover slipped. 


\subsection{Image analysis}

Photomicrographs in Figure 1 were made using a Zeiss Axioplan 2 microscope and an Evolution digital camera (MediaCybernetics). For analysis of plaque load and $\mathrm{UBB}^{+1} \mathrm{IOD}$, photographs were made using a Zeiss Axioskop microscope with Neofluor 2.5x and $5 x$ objectives and a 558,5nm bandpass filter (type DMZ-12, ITOS), connected to a Sony XC77CE CCD black and white camera. Three coronal sections per hemisphere were captured, corresponding to anterior-posterior $-1.22,-1.82$ and $-2.30 / 2.46$ relative to bregma (Paxinos and Franklin, 2001). In each section, the entire cerebral cortex, hippocampus and dentate gyrus were outlined by hand according to Paxinos et al. (Paxinos and Franklin, 2001) and analyzed with Image-Pro Plus software (version 5.1, MediaCybernetics). Measurement of sampled area volume $\left(\mathrm{mm}^{3}\right)$ and $A \beta$ plaque percentage was performed using a custom-made analysis program using Cavalieri's principle of volume estimation (Gundersen and Jensen, 1987). An example of brain area outlines and plaque determination is given in Supplementary Figure S3. $\mathrm{UBB}^{+1}$ levels were determined by measuring integrated optical densities per outlined brain area using Image-Pro Plus software. The photomicrographs taken to illustrate the astrogliosis (Figure 7) were made using an Olympus dotSlide BX microscope (Olympus) using the 10x and 20x objectives. The experimenter was blind to the genotype of the mice.

\section{$2.4 A \beta_{42}$ measurements}

Animals were decapitated and rapidly dissected on ice after which the posterior cerebral cortex was dissected and snap frozen in liquid nitrogen (Supplemental Table S2a), homogenized after defreezing using a standard lysis buffer (0.5\% Igepal, 0.01\% PMS and protease inhibitors (Complete, Roche) and with a bead-beater procedure (30s). After centrifugation at $4000 \mathrm{rpm}$ the supernatant was collected for $A \beta_{42}$ measurements using the MesoScale Discovery platform (Gaithersburg MD) measuring $A \beta_{42}$ with the antibody $6 E 10$ as a capture antibody (Supplemental Table S2a). 


\subsection{Real time PCR}

Animals were decapitated and rapidly dissected on ice after which the frontal/prefrontal cortex was snap frozen in liquid nitrogen (Supplemental Table S2b). From the frontal/prefrontal cortex mRNA was isolated according to standard protocol using Trizol/chloroform extraction (Invitrogen). Subsequently, cDNA was generated by using a first strand synthesis kit according to the manufacturers protocol (Fermentas). Samples were analyzed with the Lightcycler 480 (Roche) and matching Sybr Master (Roche) as prescribed by the manufacturer. The data were analyzed using the LinRegPCR (Ramakers et al., 2003). Two optimal housekeeping genes (Ubiquitin-40S ribosomal protein S27a (RS27a) and Elongation factor 1-alpha 1 (EF1a) (Supplemental table S3) were selected from a total of five by using the GeNorm algorithm (Warrington et al., 2000; Vandesompele et al., 2002).

\subsection{Proteasome activity measurements}

Animals were decapitated and rapidly dissected on ice after which the posterior cerebral cortex was snap frozen in liquid nitrogen (Supplemental Table S2c). Fractions enriched in proteasomes were purified from this area. The cortices were homogenized in a buffer containing 50mM Hepes- $\mathrm{KOH}$ (pH 7.5), 2mM ATP, $250 \mathrm{mM}$ sucrose and $5 \mathrm{mM} \mathrm{MgCl}_{2}$ at $4{ }^{\circ} \mathrm{C}$ after which the homogenate was centrifuged at $10,000 \times g$ for $20 \mathrm{~min}$. The supernatants were then centrifuged for $1 \mathrm{~h}$ at $100,000 \times \mathrm{g}$, transferred to another tube and subsequently centrifuged for $5 \mathrm{~h}$ at $100,000 \times \mathrm{g}$ at $4^{\circ} \mathrm{C}$. The resulting proteasome-containing pellets were dissolved in a buffer (50mM Hepes-KOH (pH 7.5), 2mM ATP, and $5 \mathrm{mM} \mathrm{MgCl}_{2}$ and $20 \%$ glycerol). To measure proteasome activity, $1 \mu \mathrm{g}$ of dissolved protein and $50 \mu \mathrm{M}$ Suc-LLVYAMC ( $N$-succinyl-Leu-Leu-Val-Tyr-7-amino-4-methyl coumarine) was used in each reaction with a total reaction volume of $30 \mu \mathrm{l}$. Each sample was measured in duplicate and corrected for a corresponding sample treated with $2 \mu \mathrm{M}$ epoxomicin. The fluorescence of the released AMC was measured in a 96-well format in real time for $1 \mathrm{~h}$ (excitation $355 \mathrm{~nm} /$ emission $450 \mathrm{~nm}$ ) using a VICTOR X3 multilabel plate reader (Perkin-Elmer). A standard curve was measured for each plate to eliminate inter-plate variability. 


\subsection{Protein quantification by Western blotting}

Animals were decapitated and rapidly dissected on ice after which the posterior cerebral cortex was dissected and snap frozen in liquid nitrogen (Supplemental Table S2d), The cortex samples used for the proteasome activity assay were diluted in lysis buffer $(0.1 \%$ SDS, $0.1 \%$ Triton-X100, 1\% glycerol, $1 \mathrm{mM}$ EDTA, $1 \mathrm{mM}$ EGTA, $1 \mathrm{mM} \mathrm{Na}_{3} \mathrm{VO}_{4}, 30 \mathrm{mM} \mathrm{NaF}$ and $1 \mathrm{x}$ Complete protease inhibitor cocktail (Roche)) to equalize total protein concentrations. The appropriate volume of $6 x$ sample buffer $(200 \mathrm{mM}$ Tris $\mathrm{HCl}(\mathrm{pH} 6.8)$ $30 \%$ glycerol, $60 \mathrm{mg} / \mathrm{ml} \beta$-mercaptoethanol and $1 \mathrm{mg} / \mathrm{ml}$ bromephenol blue) was added to the lysates to make a final concentration of $2 \mu \mathrm{g} / \mu \mathrm{l}$ total protein. Samples were heated to $95 \mathrm{C}$ for $5 \mathrm{~min}$ after which they were resolved by SDS-PAGE. Subsequently, the proteins were transferred to a nitrocellulose membrane (Bio-Rad) and immunoblotted for glial fibrillary acidic protein (GFAP) (1:1000; Biolegend clone 2E1.E9), synaptophysin (1:1000; Abcam 27354) and GAPDH (1:1.106; Fitzgerald). Primary antibodies were recognized by IRDye-labeled secondary antibodies (Rockland) which were diluted 1:10,000 and quantified by using multi-fluorescence scanning with the Odyssey ${ }^{\circledR}$ system (LI-COR).

\subsection{Assessment of ubiquitination state of $\mathrm{UBB}^{+1}$ lysine mutants}

The generation of the plasmids coding for $\mathrm{UBB}^{+1}$ and $\mathrm{UBB}^{+1} \mathrm{~K} 29,48 \mathrm{R}$ under control of the CMV promotor (pCMV plasmid) was described previously (Verhoef et al., 2009). The pCMV-UBB $^{+1}$ K63R, pCMV-UBB ${ }^{+1}$ K29,48,63R and pCMV-UBB ${ }^{+1}$ KO were generated based on the $\mathrm{UBB}^{+1}$ and $\mathrm{UBB}^{+1} \mathrm{~K} 29,48 \mathrm{R}$ plasmids by site directed mutagenesis using the QuikChange $^{\top m}$ site directed mutagenesis kit (Stratagene). All $\mathrm{UBB}^{+1}$ species were detectable by an $\mathrm{N}$-terminal myc-tag. Plasmids coding for all $\mathrm{UBB}^{+1}$ species were separately used for transfection of HeLa cells using a polyethyleneimine (PEI) based transfection protocol. The cells were harvested 24 hours post-transfection, spun down and

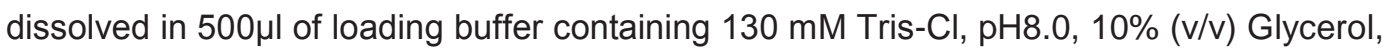
$2.3 \%(\mathrm{w} / \mathrm{v})$ SDS, $1 \%$ DTT and $0.1 \%$ brome phenol blue. Protein samples were subsequently resolved by SDS-PAGE on $16 \%$ Tris-Tricine gel (Schagger, 2006) and immunoblotted by using the monoclonal anti-myc antibody (10E9, Santa Cruz, 1:1,000) and the HRP-conjugated goat-anti-mouse antibody (GE Healthcare 1:10,000). The UBB'1 
species were detected by using ECL Plus (GE Healthcare) chemiluminescent substrate (for additional details see Figure 9).

\subsection{Statistical analyses}

For the quantitative immunohistochemistry, $A \beta_{42}$ measurements, real time PCR data, proteasome activity assays and protein quantification by immunoblotting, differences between groups were analyzed with non-parametric Kruskal-Wallis followed by MannWhitney tests and were considered significant when $p<0.05$. Statistical analysis was performed using SPSS for Windows (version 12.0.1).

For detailed information on $\operatorname{tg}$ lines and experimental design see Supplementary Figures and Supplementary Tables.

\section{Results}

\section{1 $\mathrm{UBB}^{+1}$ expression decreases $A \beta$ plaque load}

To investigate the effects of chronic $\mathrm{UBB}^{+1}$ expression on the age of onset and severity of $A \beta$ pathology, we measured the $A \beta$ plaque load in APPPS1 tg mice and in triple $\mathrm{tg}$ APPPS1/UBB ${ }^{+1}$ mice at 3, 6, 9 and 11 months of age (for group sizes, see Supplementary Table 1). A $\beta$ plaques were quantified in the cerebral cortex, dentate gyrus and the hippocampus as a whole using the $6 \mathrm{E} 10$ antibody reactive to human $A \beta$ amino acids 1-16. In these mice, plaques could also be visualized using Congo red, thioflavin-S and anti-A $\beta$ antibodies 4G8 and 6F/3D (not shown). At the age of 3 months, APPPS1 and APPPS1/UBB ${ }^{+1}$ tg mice showed a few small amyloid plaques, located mainly in the cortex (Supplementary Figure S1). From 3 months onward, plaque load increased during aging in APPPS1 and APPPS1/UBB ${ }^{+1} \mathrm{tg}$ mice (Figure 1). We previously reported that $\mathrm{UBB}^{+1} \mathrm{tg}$ mice do not show overt neuropathology (Fischer et al., 2009). Indeed, we did not detect A pathology in the $\mathrm{UBB}^{+1} \mathrm{tg}$ mice or WT control mice at any age (not shown).

Both APPPS1 and APPPS1/UBB ${ }^{+1}$ tg mice showed an increased plaque load during aging (Figures 1 and 2). However, at the age of 6 months, the plaque covered area in the cerebral cortex was significantly decreased in APPPS1/UBB ${ }^{+1} \operatorname{tg}$ mice compared to APPPS1 $\operatorname{tg}$ mice 
( $0.91 \%$ vs. $1.55 \% ; p=0.03$, Figure $2 \mathrm{~A})$. A similar result was observed in the dentate gyrus (1.06\% vs. $1.93 \% ; p=0.017$, Figure $2 \mathrm{C}$ ). The plaque covered area in the hippocampus also appeared to be decreased in APPPS1/UBB ${ }^{+1} \operatorname{tg}$ mice compared to APPPS1 $\operatorname{tg}$ mice $(0.56 \%$ vs. $0.89 \%)$, although this effect was not significant ( $p=0.126$, Figure $2 B$ ). At the ages of 9 and 11 months, the same trend towards decreased plaque load in APPPS1/UBB ${ }^{+1}$ tg mice compared to APPPS1 tg mice was present, however at these ages this effect did not reach statistical significance (Figure 2). The decreased $A \beta$ plaque load in 6-month old APPPS1/UBB ${ }^{+1} \operatorname{tg}$ mice was not caused by alterations in the volume of the sampled brain areas, which did not differ between $\mathrm{WT}, \mathrm{UBB}^{+1}$, APPPS1 or APPPS1/UBB ${ }^{+1} \operatorname{tg}$ mice at this age (Supplementary Figure S2). Also at 9 and 11 months of age, minor variations in the volume of the sampled brain regions could not account for the lower $A \beta$ accumulation in the APPPS1/UBB ${ }^{+1}$ mice (Supplementary Figure S2). These results indicate that $\mathrm{UBB}^{+1}$ decreases $A \beta$ deposition in amyloid plaques in vivo at the age of 6 months.
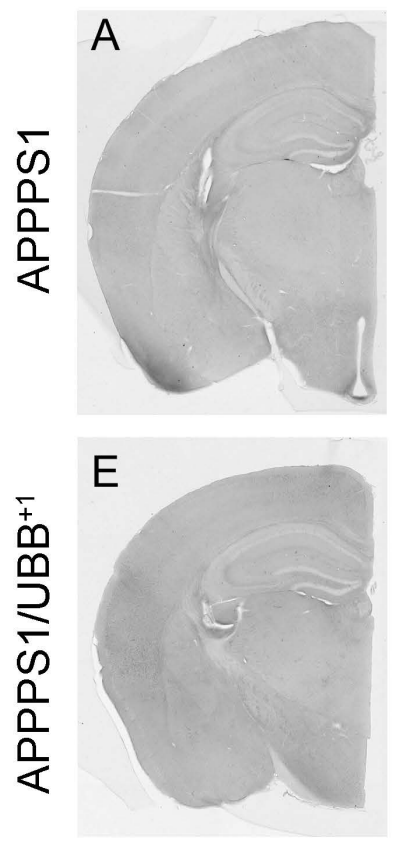

$3 \mathrm{M}$
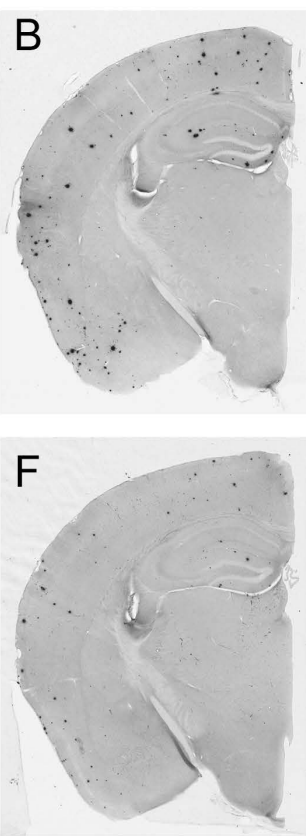

$6 \mathrm{M}$
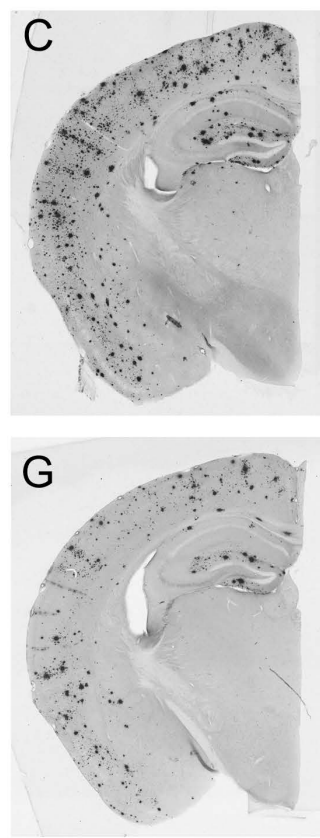

$9 \mathrm{M}$
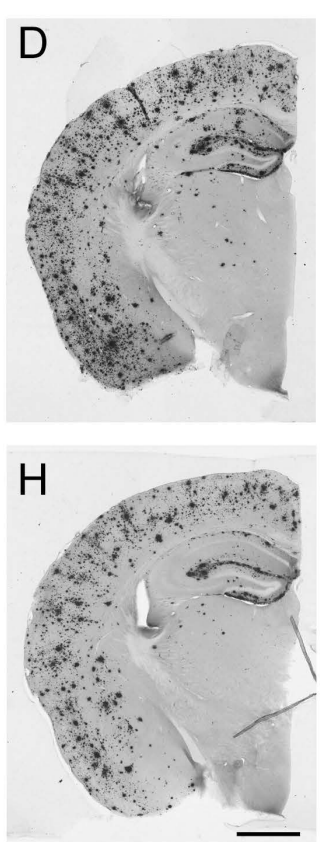

$11 \mathrm{M}$

Figure 1: A deposition in amyloid plaques in APPPS1 and APPPS1/UBB ${ }^{+1}$ tg mice. Increasing amyloid deposition during aging in the cerebral cortex, hippocampus and dentate gyrus of APPPS1 
$(A-D)$ and APPPS1/UBB ${ }^{+1}(E-H)$ tg mice. Representative low-magnification photographs of $50 \mu m$ coronal Vibratome sections of 3, 6, 9, and 11-month-old mice, stained with monoclonal anti-amyloid 6E10 antibody, Scale bar $=1 \mathrm{~mm}$.
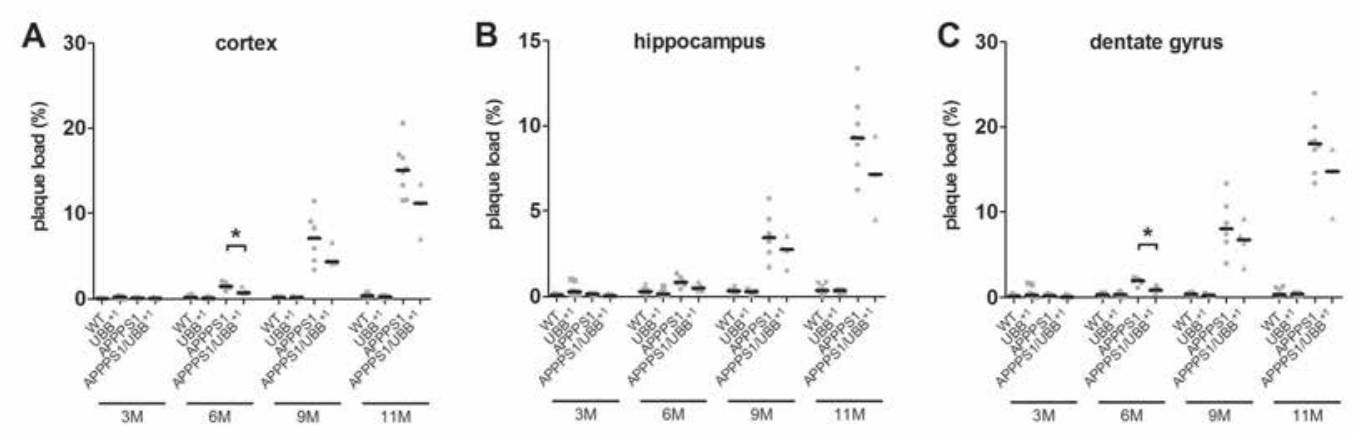

Figure 2: Age-related $U B B^{+1}$ expression and $A \beta$ plaque load. $A \beta$ plaque load in $W T, U B B^{+1}, A P P P S 1$ and APPPS1/UBB ${ }^{+1} \mathrm{tg}$ mice at the age of 3, 6, 9 and 11 months. Plaque load was determined by measuring the percentage covered by amyloid plaques in the sampled area of the cerebral cortex $(A)$, hippocampus $(B)$ and dentate gyrus (C). At 6 months of age, APPPS1/UBB ${ }^{+1}$ mice show a significantly decreased plaque load in the cortex and dentate gyrus compared to APPPS1 mice. Individual cases are plotted, median values are indicated by horizontal bars; * $p<0.05$. Please refer to Supplementary Table 1 for group sizes. 


\section{2 $\mathrm{UBB}^{+1}$ expression decreases levels of soluble $A \beta_{42}$}

The levels of insoluble $A \beta$ species, deposited in cortical amyloid plaques, differentially increase in APPPS1 and APPPS1/UBB ${ }^{+1} \operatorname{tg}$ mice during the first 6 months of life (Figures 1 and 2). To measure if this is also reflected in the level of soluble $A \beta_{42}$, we performed an electro-chemiluminescence immunoassay to determine the amount of soluble $A \beta_{42}$ in the cerebral cortex. As expected (Figure 2), we observed a significant increase in soluble $A \beta_{42}$ in the APPPS1 and APPPS1/UBB ${ }^{+1}$ tg mice, compared to WT control and $\mathrm{UBB}^{+1} \operatorname{tg}$ mice, at 3 months of age (Figure $3 A$ ). The levels of $A \beta_{42}$ were comparable between APPPS 1 and APPPS1/UBB ${ }^{+1} \operatorname{tg}$ mice (Figure $3 A$ ). The same pattern was found in 6 -months old mice (Figure $3 B$ ). However, at this age, the level of soluble $A \beta_{42}$ was significantly decreased in the APPPS1/UBB ${ }^{+1} \mathrm{tg}$ mice compared to APPPS1 mice 16.8 versus $10.2 \mu \mathrm{g} / \mathrm{mg}$ total protein, $p<0.01$ ), suggesting that the decreased cortical plaque load might be caused by lower levels of soluble $A \beta_{42}$ in the triple tg mice.
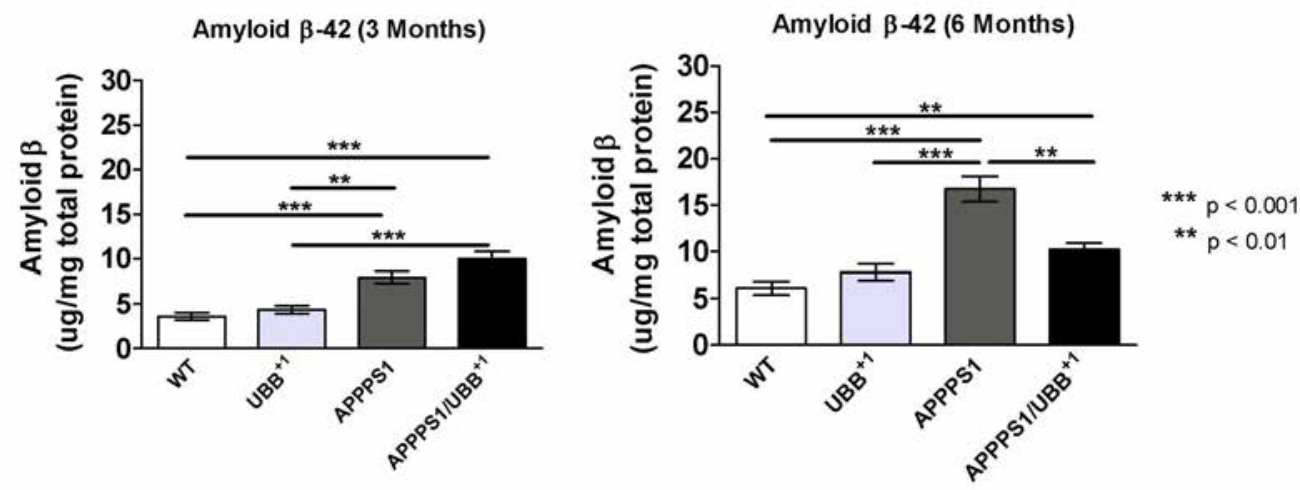

Figure 3: Age-related A 442 levels in APPPS1/UBB ${ }^{+1}$ mice. APPPS1 and APPPS1/UBB ${ }^{+1}$ tg mice show significantly increased soluble $A \beta_{42}$ levels in the posterior cerebral cortex at 3 months of age compared to $W T$ and $U B B^{+1}$ tg animals (left panel). At 6 months of age, APPPS1 tg mice have significantly elevated levels of $A \beta_{42}$ compared to all other strains. The APPPS1/UBB ${ }^{+1} \operatorname{tg}$ mice express significantly more $A \beta_{42}$ compared to $W T$ and single $U B B^{+1}$ tg mice, but significantly lower levels than the APPPS1 line (right panel). Please refer to Supplementary Table 1 for group sizes. 


\subsection{Amyloid deposition does not affect proteolytic turnover of UBB ${ }^{+1}$}

$A \beta$ has been reported to decrease proteasome function in an APPSwe tg mouse model of $A D$ (Oh et al., 2005; Almeida et al., 2006). Because $\mathrm{UBB}^{+1}$ is a substrate for degradation by the UPS (Lindsten et al., 2002;van Tijn et al., 2007), we investigated whether $A \beta$ deposition in plaques also affected accumulation of $\mathrm{UBB}^{+1}$ in $A P P P S 1 / \mathrm{UBB}^{+1} \operatorname{tg}$ mice. We quantified the $\mathrm{UBB}^{+1}$ protein levels by immunohistochemistry in the same APPPS1/UBB ${ }^{+1} \operatorname{tg}$ mice used for the plaque load measurements, and compared these levels to $\mathrm{UBB}^{+1}$ in single $\mathrm{UBB}^{+1} \operatorname{tg}$ mice. Neuronal $\mathrm{UBB}^{+1}$ staining was present in the $\mathrm{UBB}^{+1} \operatorname{tg}$ mice mainly in the cerebral cortex, dentate gyrus, and hippocampus, as shown for the age of 11 months in Figure 4 (Fischer et al., 2009). In APPPS1/UBB ${ }^{+1}$ tg mice, a similar staining pattern was observed, and APPPS1 tg and WT mice did not express UBB ${ }^{+1}$, as would be expected (Figure 4). We previously reported that $\mathrm{UBB}^{+1}$ protein is maximally expressed from postnatal day 22 onwards (Fischer et al., 2009). Indeed, the $\mathrm{UBB}^{+1}$ levels in the cortex and hippocampus remained constant up to 11 months of age (Figure 5). Furthermore, we did not observe any significant differences in the levels of $\mathrm{UBB}^{+1}$ expression between $\mathrm{UBB}^{+1}$ and APPPS1/UBB ${ }^{+1} \operatorname{tg}$ mice at any age examined (Figure 5). These results show that in $A P P P S 1 / U^{\prime} B^{+1} \operatorname{tg}$ mice, $A \beta$ accumulation does not induce additional accumulation of $\mathrm{UBB}^{+1}$.

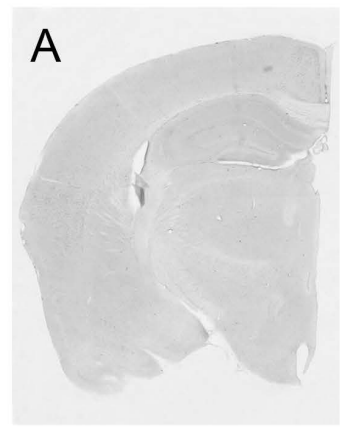

WT

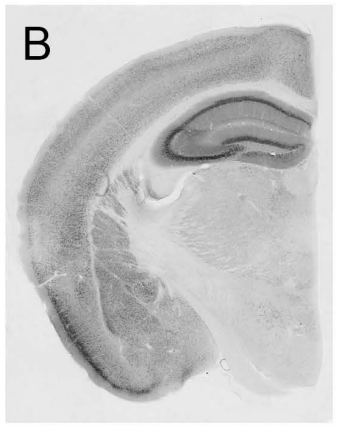

$\mathrm{UBB}^{+1}$

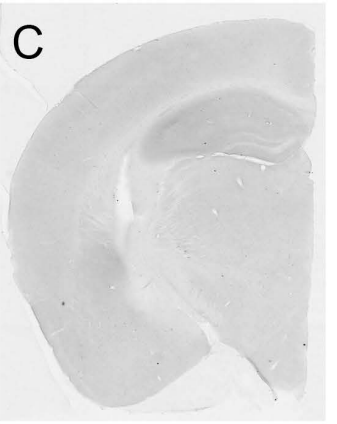

APPPS1

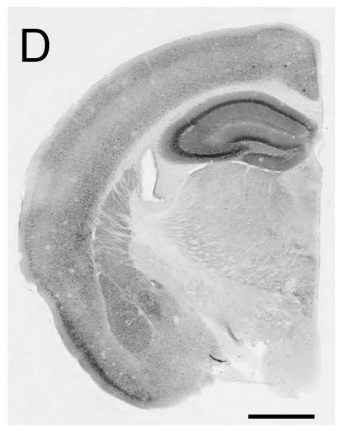

APPPS1/UBB ${ }^{+1}$

Figure 4: $U B B^{+1}$ protein expression in the mouse brains at 11 months of age. Representative lowmagnification photographs of $50 \mu \mathrm{m}$ coronal Vibratome sections of 11 month-old WT (A), UBB ${ }^{+1}(B)$, APPPS1 (C) and APPPS1/UBB ${ }^{+1}(D)$ mice were stained with polyclonal Ubi3 antibody against the unique $C$-terminus of $U B B^{+1}$, Scale bar $=1 \mathrm{~mm}$. 


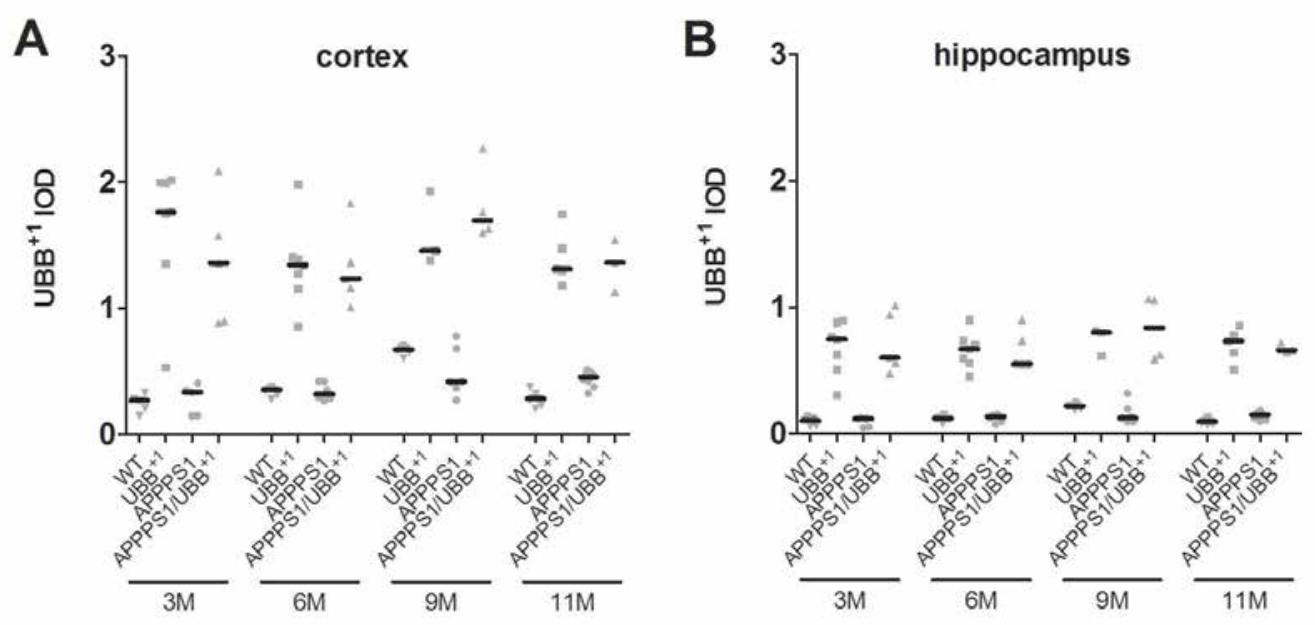

Figure 5: $A \beta$ accumulation does not induce $U B B^{+1}$ accumulation. $U B B^{+1}$ levels were measured in the cortex (A) and hippocampus (B) of WT, UBB ${ }^{+1}, A P P P S 1$ and APPPS1/UBB ${ }^{+1}$ tg mice at 3, 6, 9 and 11 months of age. UBB ${ }^{+1}$ protein levels were determined by measuring the integrated optical density (IOD) per sampled brain area. No significant differences were present at any age. Individual cases are plotted; median values are indicated by horizontal bars. 


\subsection{Gene expression profiles of UBB ${ }^{+1}, \operatorname{tg}$ APP, GFAP and BACE1}

To determine if the reduced $A \beta$ deposition observed in APPPS1/UBB ${ }^{+1} \operatorname{tg}$ mice could be attributable to changes in transgene mRNA expression, we measured transgene transcript levels at 3 and 6 months of age. UBB ${ }^{+1}$ mRNA was equally expressed in all mice carrying the $\mathrm{UBB}^{+1}$ transgene irrespective of age (Figure 6). This is in concordance with the unaltered levels of $\mathrm{UBB}^{+1}$ protein in the cortex at these ages (Figure 5A). Using a primer set specifically detecting the mouse/human APPSwe transcript (tg APP) and not endogenous mouse APP, we show that APPPS1/UBB ${ }^{+1}$ tg mice expressed $29 \%$ more tg APP mRNA compared to APPPS1 alone at 3 months of age (Figure 6). However, at 6 months of age the expression levels of $\operatorname{tg}$ APP were comparable (Figure 6) and altered transgene expression therefore does not underlie the observed changes in $A \beta$ levels at this age. In order to assess astrogliosis in the APPPS1/UBB ${ }^{+1}$ tg mice, we measured GFAP mRNA expression. At the age of 6 months, APPPS1/UBB ${ }^{+1}$ tg mice had significant more GFAP mRNA (63\%) compared to WT and $\mathrm{UBB}^{+1}$ tg mice, indicating that there is some degree of neuroinflammation in the APPPS1/UBB ${ }^{+1}$ mice at 6 months of age (Figure 6). The APPPS1 tg mice showed only a trend towards an increase of GFAP mRNA when compared to WT mice $(p=0.07)$. However, APPPS1/UBB ${ }^{+1}$ mice did not show significantly more GFAP mRNA expression than APPPS1 mice. The transcript level of endogenous $B A C E 1$, the $\beta$-secretase enzyme essential for generating $A \beta$ peptides from full length APP, was unchanged at the ages studied (Figure 6). 

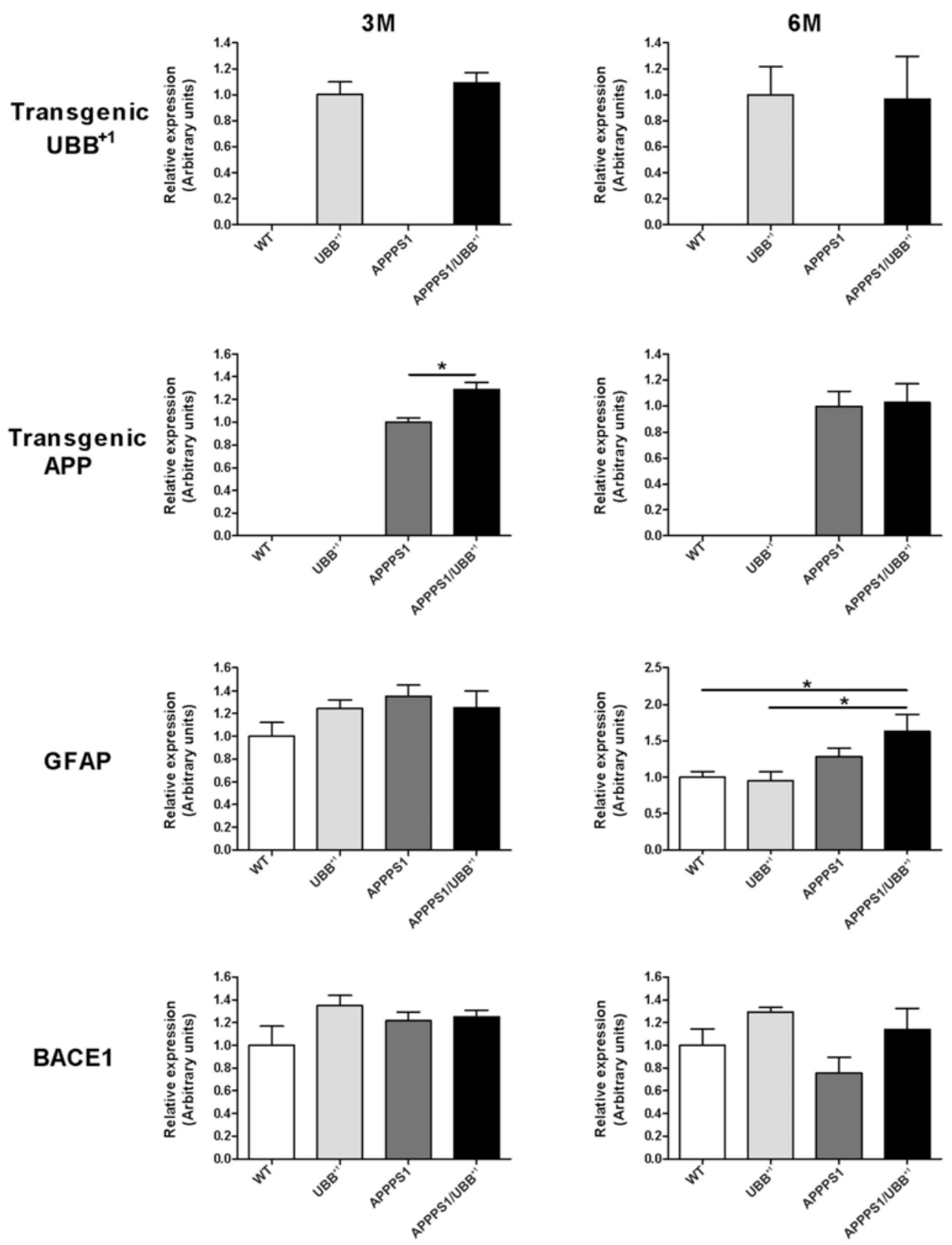
Figure 6: Quantification of $m R N A$ coding for $U B B^{+1}$, $\operatorname{tg} A P P, G F A P$ and BACE1. Transcripts coding for $U B B^{+1}$, tg APP, GFAP and BACE1 were measured in the (pre)frontal cortex of $W T$, UBB ${ }^{+1}$, APPPS1 and APPPS1/UBB ${ }^{+1}$ mice at 3 and 6 months of age. Tg UBB ${ }^{+1}$ and BACE 1 mRNA levels are unchanged in any transgene at any time point (top and bottom row). APPPS1 mice express lower levels of $t g$ APP mRNA compared to the APPPS1/UBB ${ }^{+1}$ tg mice at 3 months of age (second row). This difference is absent at 6 months of age. GFAP expression is increased in the APPPS1/UBB ${ }^{+1}$ tg animals compared to WT and $U B B^{+1}$ tg animals (third row) at the age of 6 months. Mean expression levels are plotted, error bars indicate the SEM. * $p<0.05$

\subsection{GFAP is increased in the APPPS1/UBB ${ }^{+1}$ transgenic mouse}

We quantified levels of GFAP protein in order to assess the level of astrogliosis in $\mathrm{UBB}^{+1}$, APPPS1 and APPPS1/UBB ${ }^{+1} \mathrm{tg}$ mouse lines compared to WT mice. At 3 months of age no differences could be observed in GFAP expression in any of the $\mathrm{tg}$ mouse lines compared to control (Figure 7A, B). In concordance with the increased GFAP mRNA levels in the APPPS1/UBB ${ }^{+1}$ tg mice at 6 months of age (Figure 6), GFAP protein was significantly increased in these mice when compared to WT mice and $\mathrm{UBB}^{+1} \operatorname{tg}$ animals (Figure $7 \mathrm{C}$ ), which was also reflected when stained for GFAP immunohistochemically (Figure 7D). We also analysed synaptophysin at 3 and 6 months of age to assess whether the decreased $A \beta$ levels and increased astrogliosis affected synaptic vesicle function. However, we did not find a significant difference in any of these lines compared to WT controls (Supplemental Figure 4). 


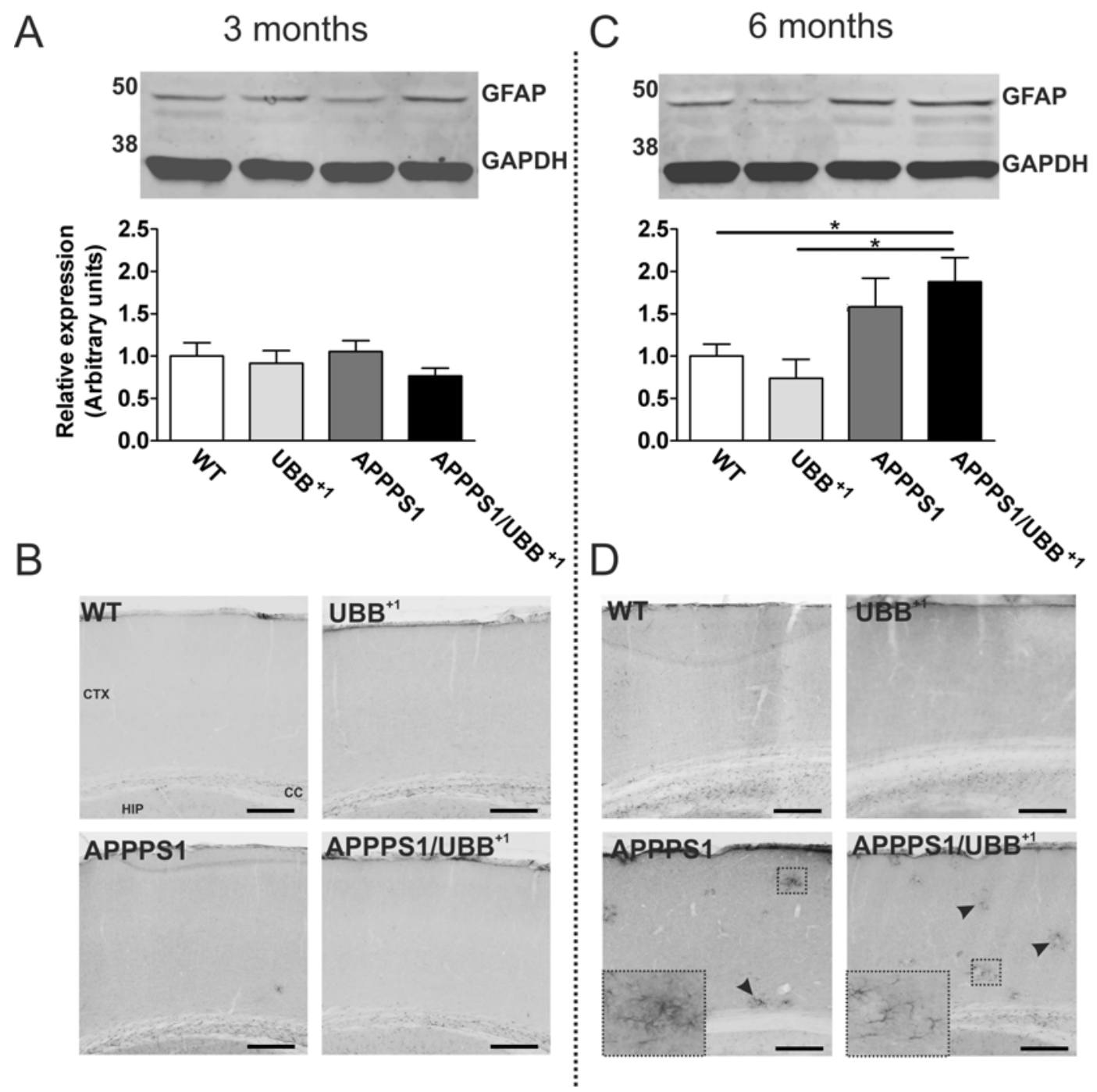

Figure 7: Cortical GFAP protein expression. At 3 months of age, the expression of GFAP protein did not differ between $W T$, UBB ${ }^{+1}$, APPPS1 and APPPS1/UBB ${ }^{+1}$ tg mice ( $A$ and $B$ ). Photomicrographs were taken of the cerebral cortex (CTX), hippocampus (HIP) and the corpus callosum (CC). At 6 months of age, The APPPS1/UBB ${ }^{+1}$ show significantly higher expression of GFAP when compared to the $U B B^{+1}$ tg and WT mice (C). Immunohistochemical staining confirms these data showing local activation of astrocytes expressing GFAP (enlarged insert) in the cortex of APPPS1 and APPPS1/UBB ${ }^{+1} \operatorname{tg}$ mice (D) ( $n=2$ of each genotype), scale bar $=200 \mu \mathrm{m}$. 


\subsection{Decreased $A \beta$ deposition in APPPS1/UBB ${ }^{+1}$ transgenic mice is not caused by proteasome inhibition}

We previously reported a modest decline in chymotrypsin-like proteasome activity in $\mathrm{UBB}^{+1}$ $\operatorname{tg}$ mice at 8 months of age to $82 \%$ of WT levels (Fischer et al., 2009). It is thus conceivable that alterations in proteasome function may also play a role in the observed decline in $A \beta_{42}$ levels and amyloid plaque burden at 6 months of age in the APPPS1/UBB ${ }^{+1}$ triple tg mice compared to APPPS1 tg mice. Therefore, we measured chymotryptic-like activity of partly purified proteasomes from WT control mice and from the $\mathrm{UBB}^{+1}$, APPPS1 and APPPS1/UBB ${ }^{+1} \operatorname{tg}$ mouse lines at 3 and 6 months of age. We could not detect significant differences in proteasome activity in any of the tg mouse lines compared to the WT background strain at either time point (Figure 8). These data show that proteasome catalytic activity is not affected by amyloid accumulation in the APPPS1 mice at these ages. This is in concordance with previous findings in Tg2576 tg mice, overexpressing the APPSwe transgene, which show significantly decreased chymotryptic proteasome activity only from 15 months onwards (Oh et al., 2005). Surprisingly, expression of $\mathrm{UBB}^{+1}$ did not lead to proteasome inhibition at the age of 6 months in the single $\mathrm{UBB}^{+1} \operatorname{tg}$ line. Additional expression of $\mathrm{UBB}^{+1}$ in the APPPS1/UBB ${ }^{+1}$ mice did also not lead to decreased proteasome activity compared to the $\mathrm{UBB}^{+1}$ or APPPS1 line, suggesting that the decreased $A \beta$ in the triple tg line is not dependent on chymotryptic proteasome activity.
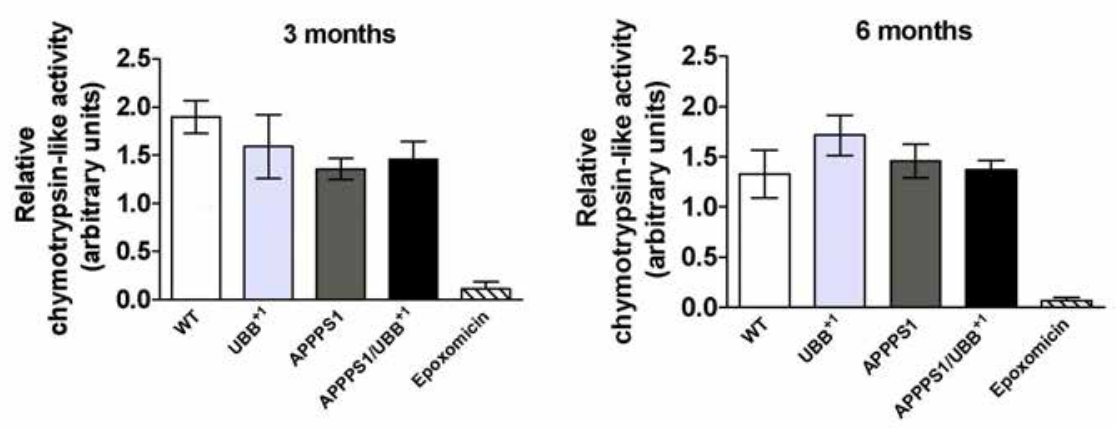
Figure 8: Cortical chymotryptic-like proteasome activity. Chymotryptic-like proteasome activity was measured in the posterior cerebral cortex from WT $(A), U_{B B}^{+1}(B), A P P P S 1(C)$ and APPPS1/UBB ${ }^{+1}$ (D) tg mice at 3 and 6 months of age. Note the lack of significant differences between all groups.

\subsection{Ubiquitination of $\mathrm{UBB}^{+1}$ involves $\mathrm{K} 63$ linkages}

Recently it was published that unanchored K63 linked polyubiquitin chains are involved in regulation of the nuclear factor $\mathrm{k} \beta$ (NFkB) pathway independent of proteasomal function (Xia et al., 2009). Although the extreme C-terminus of $\mathrm{UBB}^{+1}$ has been altered most of amino acids (75 out of 76 ) are still identical to ubiquitin. The possibility of ubiquitination of $\mathrm{UBB}^{+1}$ at the conserved K63 has not been studied previously. As can be seen in Figure 9, $\mathrm{UBB}^{+1}$ can be ubiquitinated at $\mathrm{K} 63$ thus resulting in unanchored $\mathrm{K} 63$ linked polyubiquitin chains. Both K63 linked monoubiquitination and polyubiquitination (see lane K29, 48R) can be observed of $\mathrm{UBB}^{+1}\left(\mathrm{UB}-\mathrm{UBB}^{+1}\right)$ can be observed. No difference in ubiquitinated species can be observed between the $\mathrm{UBB}^{+1} \mathrm{~K} 29,48,63 \mathrm{R}$ triple mutant and complete lysine less $\mathrm{UBB}^{+1} \mathrm{KO}$ (all lysines within $\mathrm{UBB}^{+1}$ are modified to Arg) indicating that only the lysine's 29 , 48 and 63 within the $\mathrm{UBB}^{+1}$ protein can be used for ubiquitination. On the contrary lysine's at positions $6,11,27$ and 33 of $\mathrm{UBB}^{+1}$ are not ubiquitinated since no additional monoubiquitin can be observed when compared to lysine less $\mathrm{UBB}^{+1} \mathrm{KO}$. This opens the possibility that unanchored polyubiquitin $\mathrm{K} 63$ linked chains of $\mathrm{UBB}^{+1}$ may alter cell homeostasis. 


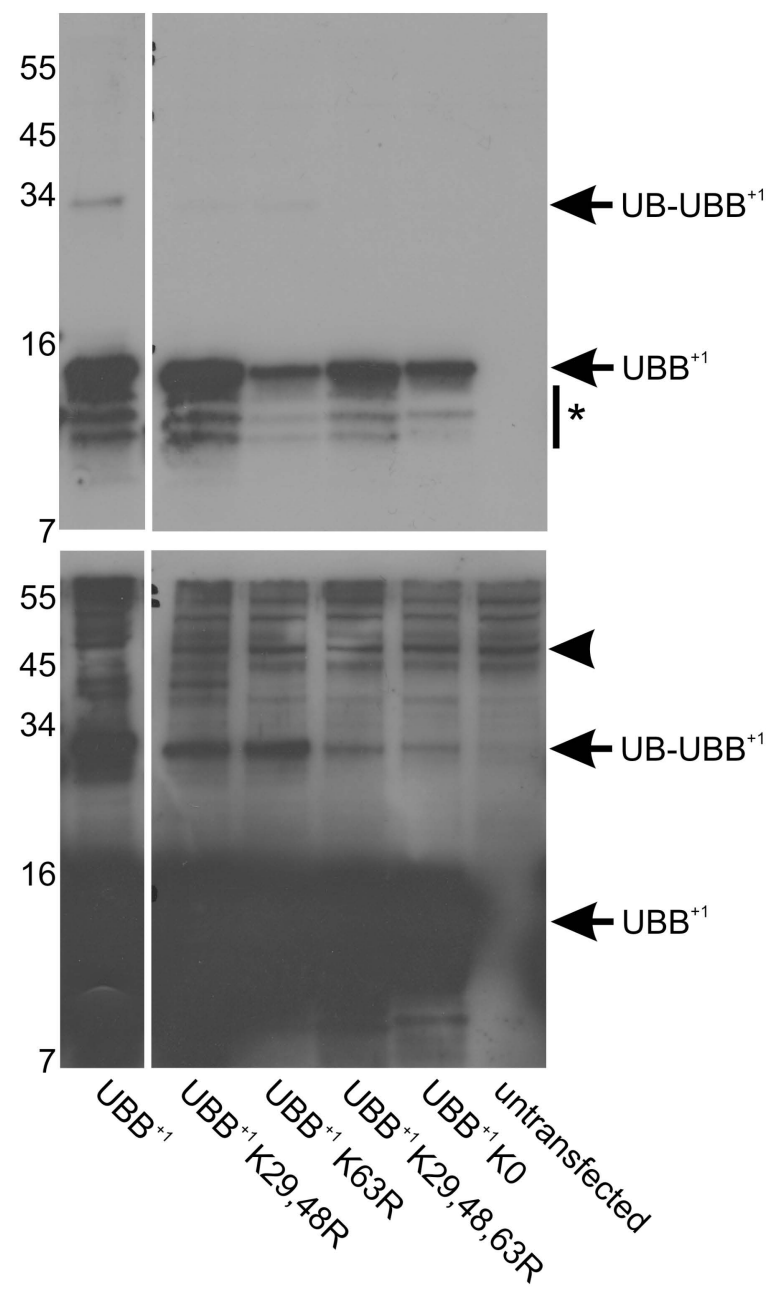

Figure 9: Ubiquitination of $U B B^{+1}$ at K63. Immunoblot showing myc-tagged $U B B^{+1}$ species expressed in HeLa cells by transient transfection. $\mathrm{UBB}^{+1}$ is ubiquitinated (UB-UBB ${ }^{+1}$ ) and truncated (*, (Dennissen et al., 2011)) (top panel, lane 1). In the high exposure image (lower panel) the ubiquitinated $\mathrm{UBB}^{+1}$ species are more obvious. Ubiquitination of $U B B^{+1}$ can be reduced strongly by substituting lysines 29 and 48 into arginine (lane 2). Substitution of lysine 63 into arginine also reduces the quantity of ubiquitinated species (lane 3). Substitution of lysine 29, 48 and 63 reduces ubiquitinated species even further (lane 4) to levels similar to lysine less UBB ${ }^{+1}$ (UBB ${ }^{+1} \mathrm{KO}$, lane 5). $U B B^{+1} K O$ still shows some residual ubiquitination possibly due to $N$-terminally ubiquitination. Untransfected cells do not show any $U B B^{+1}$ species (lane 6). The arrowhead in the high exposure image indicates an aspecifically stained band which serves as a loading control. 


\section{Discussion}

In the present study, we found an effect of $\mathrm{UBB}^{+1}$ on $\mathrm{A} \beta$ plaque formation in vivo. That is, $A \beta$ deposition in plaques as well as soluble $A \beta_{42}$ levels were decreased in 6 month old APPPS1/UBB ${ }^{+1} \operatorname{tg}$ mice compared to 6 month old APPPS1 mice, indicating that $\mathrm{UBB}^{+1}$ can reduce amyloid deposition. This decrease is possibly caused by a proteasome independent mechanism. The age of onset of $A \beta$ deposition was unaffected, as $A \beta$ plaques were detected at 3 months of age in both APPPS1 and APPPS1/UBB ${ }^{+1} \operatorname{tg}$ mice. Using different staining methods, others reported detection of plaques at 4 (Garcia-Alloza et al., 2006; Ruan et al., 2009) or 6 months of age (Jankowsky et al., 2004) in APPPS1 mice. In concordance with these studies, the cerebral cortex and hippocampus were the brain regions mainly affected.

To study the effects of $\mathrm{UBB}^{+1}$ during the critical period of $A \beta$ plaque formation (Tucker et al., 2008) we focused our studies on mice aged 3 and 6 months. At 3 and 6 months of age no decrease in proteasomal chymotrypsin-like activity in $\mathrm{UBB}^{+1}$ line 3413 was found, whereas a moderate, but significant proteasome impairment was found in these mice at 8 months of age (Fischer et al., 2009), suggesting that proteasome inhibition in this line is age-dependent. A similar age-dependent decrease in proteasomal chymotrypsin-like activity was found in an APPSwe tg mouse line (Oh et al., 2005). Also in the APPPS1 and APPPS1/UBB ${ }^{+1}$ tg lines, the chymotryptic-like proteasome activity was comparable to WT levels, showing that the reduction of $A \beta$ plaque load in the APPPS1/UBB ${ }^{+1}$ line we measured at 6 months cannot be attributed to proteasomal inhibition. Since reduced proteasome function did not account for the decrease in plaque load in the APPPS1/UBB ${ }^{+1}$ it is not yet clear which mechanism is responsible for this observation. It is known that K63linked polyubiquitin chains are a feature of neurodegeneration (Lim and Lim, 2011) and it was suggested that $K 63$ linkages play a role in AD (Paine et al., 2009;Dammer et al., 2011) and promotes as a common denominator the formation and autophagic clearance of protein inclusions (Tan et al., 2008). Indeed we showed that $\mathrm{UBB}^{+1}$ is ubiquitinated not only at K29 and K48 as reported previously (Lindsten et al., 2002), but also at K63. The K63linked ubiquitination of $\mathrm{UBB}^{+1}$ would give rise to an unanchored $\mathrm{K} 63$ linked polyubiquitin chain. These unanchored polyubiquitin chains are able to regulate directly the NFKB 
pathway (Xia et al., 2009). Thus it is possible that $\mathrm{UBB}^{+1}$ can alter cell homeostasis and possibly $A \beta$ deposition by (poly-) ubiquitination at $\mathrm{K} 63$ of $\mathrm{UBB}^{+1}$ through an inflammatory feedback loop or changes in autophagy. Our data are in agreement with the recent hypothesis on $\mathrm{UBB}^{+1}$ ubiquitination at $\mathrm{K} 63$ and harmful effects through NFKB activity or NFKB-mediated neuroinflammatory processes (Chadwick et al., 2012), as measurement of ATG8 and ATG12 did not show a difference in the $\mathrm{UBB}^{+1}$ transgenic mice (data not shown). To further study possible mechanisms of diminished $A \beta$ plaque formation we measured gene expression levels of several genes relevant to amyloid deposition. For example, mRNA levels of $\operatorname{tg}$ APP are increased in the APPPS1/UBB ${ }^{+1} \mathrm{tg}$ mice of 3 months of age. However, the levels are comparable at 6 months of age when a significant decrease in $A \beta$ plaques and soluble $A \beta_{42}$ is present. Regarding another possible mechanism, neuronal $\beta$-secretase cleavage of APP is predominantly performed by BACE1 (Cai et al., 2001). In keeping with this, administration of BACE1 inhibitor reduces $A \beta$ production in APPSwe/PS1 ${ }^{\mathrm{M} 146 \mathrm{~V}}$ tg mice (Hussain et al., 2007) and BACE1 knockout prevents amyloid pathology in several AD tg models (e.g., (McConlogue et al., 2007)), indicating that $\beta$ secretase cleavage is the rate-limiting step for $A \beta$ formation in vivo. Nonetheless, the significant changes in A $\beta$ aggregation at 6 months in the APPPS1/UBB ${ }^{+1}$ line in our study cannot be explained by BACE1 mRNA changes, as these are comparable to expression levels in APPPS1 mice.

Proteasome function as shown by chymotrypsin-like activity was unaltered in $\mathrm{UBB}^{+1}$, APPPS1 or APPPS1/UBB ${ }^{+1} \mathrm{tg}$ mice compared to controls at 3 and 6 months of age indicating that astrogliosis in the APPPPS1/UBB ${ }^{+1} \mathrm{tg}$ mice at 6 months of life does not directly correlate with cortical proteasome activity. At 6 months of age we saw an increased expression of GFAP mRNA and protein levels in the APPPPS1/UBB ${ }^{+1} \mathrm{tg}$ mice compared to controls while a difference is not significant in APPPS1 tg mice. The latter observation is in agreement with a report that shows progressive plaque-associated astrogliosis in APPPS1 tg mice starts only from 6 months onwards (Ruan et al., 2009). Thus, APPPPS1/UBB ${ }^{+1} \mathrm{tg}$ mice show a precocious increase in astrogliosis and inflammation suggesting that although $A \beta$ neuropathology is reduced, neuroinflammation is already increased. However, this neuroinflammation does not seem to have a significant effect on 
synapse formation because synaptophysin levels do not differ between the tg mouse lines and controls at 3 or 6 months of age.

\section{Conclusion}

Exposure to $\mathrm{UBB}^{+1}$ results in decreased deposition of $A \beta$ in amyloid plaques and reduced levels of soluble $A \beta_{42}$ at 6 months and it transient. The difference in plaque load cannot be explained by differences in tg APP mRNA levels, changed expression of $\beta$-secretase or reduced proteasome function. The precise mechanism by which $\mathrm{UBB}^{+1}$ may decrease plaque load remains to be determined, as both APP processing as well as A $\beta$ clearance could be affected. As we found that $\mathrm{UBB}^{+1}$ can be ubiquitinated at $\mathrm{K} 63$, non-proteolytic actions of ubiquitinated $\mathrm{UBB}^{+1}$ are possibly causing this decrease in plaque load. Further insights into these mechanisms can contribute to understanding the role of the UPS in the pathogenesis of $A D$. 


\section{References}

Almeida, C.G., Takahashi, R.H., and Gouras, G.K. (2006). Beta-amyloid accumulation impairs multivesicular body sorting by inhibiting the ubiquitin-proteasome system. Journal of Neuroscience 26, 4277-4288.

Cai, H., Wang, Y., Mccarthy, D., Wen, H., Borchelt, D.R., Price, D.L., and Wong, P.C. (2001). BACE1 is the major beta-secretase for generation of Abeta peptides by neurons. Nature Neuroscience 4, 233-234.

Chadwick, L., Gentle, L., Strachan, J., and Layfield, R. (2012). Review: Unchained maladie - a reassessment of the role of $\mathrm{Ubb}(+1)$-capped polyubiquitin chains in Alzheimer's disease. Neuropathol Appl Neurobiol 38, 118-131.

Christie, G., Markwell, R.E., Gray, C.W., Smith, L., Godfrey, F., Mansfield, F., Wadsworth, H., King, R., Mclaughlin, M., Cooper, D.G., Ward, R.V., Howlett, D.R., Hartmann, T., Lichtenthaler, S.F., Beyreuther, K., Underwood, J., Gribble, S.K., Cappai, R., Masters, C.L., Tamaoka, A., Gardner, R.L., Rivett, A.J., Karran, E.H., and Allsop, D. (1999). Alzheimer's disease: correlation of the suppression of beta-amyloid peptide secretion from cultured cells with inhibition of the chymotrypsin-like activity of the proteasome. J Neurochem 73, 195-204.

Ciechanover, A., and Brundin, P. (2003). The ubiquitin proteasome system in neurodegenerative diseases: sometimes the chicken, sometimes the egg. Neuron 40, 427-446.

Dammer, E.B., Na, C.H., Xu, P., Seyfried, N.T., Duong, D.M., Cheng, D., Gearing, M., Rees, H., Lah, J.J., Levey, A.I., Rush, J., and Peng, J. (2011). Polyubiquitin linkage profiles in three models of proteolytic stress suggest the etiology of Alzheimer disease. J Biol Chem 286, 1045710465.

Dennissen, F.J., Kholod, N., Hermes, D.J., Kemmerling, N., Steinbusch, H.W., Dantuma, N.P., and Van Leeuwen, F.W. (2011). Mutant ubiquitin $\left(\mathrm{UBB}^{+1}\right)$ associated with neurodegenerative disorders is hydrolyzed by ubiquitin C-terminal hydrolase L3 (UCH-L3). FEBS Lett 585, 2568-2574.

Fischer, D.F., De Vos, R.A., Van Dijk, R., De Vrij, F.M., Proper, E.A., Sonnemans, M.A., Verhage, M.C., Sluijs, J.A., Hobo, B., Zouambia, M., Steur, E.N., Kamphorst, W., Hol, E.M., and Van Leeuwen, F.W. (2003). Disease-specific accumulation of mutant ubiquitin as a marker for proteasomal dysfunction in the brain. FASEB Journal 17, 2014-2024.

Fischer, D.F., Van Dijk, R., Van Tijn, P., Hobo, B., Verhage, M.C., Van Der Schors, R.C., Li, K.W., Van Minnen, J., Hol, E.M., and Van Leeuwen, F.W. (2009). Long-term proteasome dysfunction in the mouse brain by expression of aberrant ubiquitin. Neurobiology of Aging 30, 847-863.

Flood, F., Murphy, S., Cowburn, R.F., Lannfelt, L., Walker, B., and Johnston, J.A. (2005). Proteasome-mediated effects on amyloid precursor protein processing at the gammasecretase site. Biochem J 385, 545-550.

Garcia-Alloza, M., Robbins, E.M., Zhang-Nunes, S.X., Purcell, S.M., Betensky, R.A., Raju, S., Prada, C., Greenberg, S.M., Bacskai, B.J., and Frosch, M.P. (2006). Characterization of amyloid deposition in the APPswe/PS1dE9 mouse model of Alzheimer disease. Neurobiol Dis 24, 516-524. 
Gregori, L., Fuchs, C., Figueiredo-Pereira, M.E., Van Nostrand, W.E., and Goldgaber, D. (1995). Amyloid beta-protein inhibits ubiquitin-dependent protein degradation in vitro. Journal of Biological Chemistry 270, 19702-19708.

Gundersen, H.J., and Jensen, E.B. (1987). The efficiency of systematic sampling in stereology and its prediction. J Microsc 147, 229-263.

Hardy, J. (2009). The amyloid hypothesis for Alzheimer's disease: a critical reappraisal. Journal of Neurochemistry 110, 1129-1134.

Hardy, J., and Selkoe, D.J. (2002). The amyloid hypothesis of Alzheimer's disease: progress and problems on the road to therapeutics. Science 297, 353-356.

Hussain, I., Hawkins, J., Harrison, D., Hille, C., Wayne, G., Cutler, L., Buck, T., Walter, D., Demont, E., Howes, C., Naylor, A., Jeffrey, P., Gonzalez, M.I., Dingwall, C., Michel, A., Redshaw, S., and Davis, J.B. (2007). Oral administration of a potent and selective non-peptidic BACE-1 inhibitor decreases beta-cleavage of amyloid precursor protein and amyloid-beta production in vivo. Journal of Neurochemistry 100, 802-809.

Jankowsky, J.L., Fadale, D.J., Anderson, J., Xu, G.M., Gonzales, V., Jenkins, N.A., Copeland, N.G., Lee, M.K., Younkin, L.H., Wagner, S.L., Younkin, S.G., and Borchelt, D.R. (2004). Mutant presenilins specifically elevate the levels of the 42 residue beta-amyloid peptide in vivo: evidence for augmentation of a 42-specific gamma secretase. Human molecular genetics $13,159-170$.

Jankowsky, J.L., Slunt, H.H., Ratovitski, T., Jenkins, N.A., Copeland, N.G., and Borchelt, D.R. (2001). Co-expression of multiple transgenes in mouse CNS: a comparison of strategies. Biomol Eng 17, 157-165.

Keller, J.N., Hanni, K.B., and Markesbery, W.R. (2000). Impaired proteasome function in Alzheimer's disease. Journal of Neurochemistry 75, 436-439.

Kienlen-Campard, P., Feyt, C., Huysseune, S., De Diesbach, P., N'kuli, F., Courtoy, P.J., and Octave, J.N. (2006). Lactacystin decreases amyloid-beta peptide production by inhibiting betasecretase activity. J Neurosci Res 84, 1311-1322.

Ko, S., Kang, G.B., Song, S.M., Lee, J.G., Shin, D.Y., Yun, J.H., Sheng, Y., Cheong, C., Jeon, Y.H., Jung, Y.K., Arrowsmith, C.H., Avvakumov, G.V., Dhe-Paganon, S., Yoo, Y.J., Eom, S.H., and Lee, W. (2010). Structural basis of E2-25K/UBB ${ }^{+1}$ interaction leading to proteasome inhibition and neurotoxicity. J Biol Chem 285, 36070-36080.

Lim, K.L., and Lim, G.G. (2011). K63-linked ubiquitination and neurodegeneration. Neurobiol Dis 43, 9-16.

Lindsten, K., De Vrij, F.M., Verhoef, L.G., Fischer, D.F., Van Leeuwen, F.W., Hol, E.M., Masucci, M.G., and Dantuma, N.P. (2002). Mutant ubiquitin found in neurodegenerative disorders is a ubiquitin fusion degradation substrate that blocks proteasomal degradation. Journal of Cell Biology 157, 417-427.

Lopez Salon, M., Morelli, L., Castano, E.M., Soto, E.F., and Pasquini, J.M. (2000). Defective ubiquitination of cerebral proteins in Alzheimer's disease. Journal of Neuroscience Research 62, 302-310.

Mcconlogue, L., Buttini, M., Anderson, J.P., Brigham, E.F., Chen, K.S., Freedman, S.B., Games, D., Johnson-Wood, K., Lee, M., Zeller, M., Liu, W., Motter, R., and Sinha, S. (2007). Partial reduction of BACE1 has dramatic effects on Alzheimer plaque and synaptic pathology in APP Transgenic Mice. Journal of Biological Chemistry 282, 26326-26334. 
Nicklas, W., Baneux, P., Boot, R., Decelle, T., Deeny, A.A., Fumanelli, M., and Illgen-Wilcke, B. (2002). Recommendations for the health monitoring of rodent and rabbit colonies in breeding and experimental units. Laboratory animals 36, 20-42.

Nixon, R.A., Yang, D.S., and Lee, J.H. (2008). Neurodegenerative lysosomal disorders: a continuum from development to late age. Autophagy 4, 590-599.

Nunan, J., Shearman, M.S., Checler, F., Cappai, R., Evin, G., Beyreuther, K., Masters, C.L., and Small, D.H. (2001). The C-terminal fragment of the Alzheimer's disease amyloid protein precursor is degraded by a proteasome-dependent mechanism distinct from gammasecretase. Eur J Biochem 268, 5329-5336.

Oh, S., Hong, H.S., Hwang, E., Sim, H.J., Lee, W., Shin, S.J., and Mook-Jung, I. (2005). Amyloid peptide attenuates the proteasome activity in neuronal cells. Mechanisms of Ageing and Development 126, 1292-1299.

Paine, S., Bedford, L., Thorpe, J.R., Mayer, R.J., Cavey, J.R., Bajaj, N., Sheppard, P.W., Lowe, J., and Layfield, R. (2009). Immunoreactivity to Lys63-linked polyubiquitin is a feature of neurodegeneration. Neurosci Lett 460, 205-208.

Paxinos, G., and Franklin, K.B.J. (2001). The mouse brain in stereotaxic coordinates. San Diego, CA: Academic Press.

Ramakers, C., Ruijter, J.M., Deprez, R.H., and Moorman, A.F. (2003). Assumption-free analysis of quantitative real-time polymerase chain reaction (PCR) data. Neuroscience Letters 339, 6266.

Ruan, L., Kang, Z., Pei, G., and Le, Y. (2009). Amyloid deposition and inflammation in APPswe/PS1dE9 mouse model of Alzheimer's disease. Curr Alzheimer Res 6, 531-540.

Schagger, H. (2006). Tricine-SDS-PAGE. Nat Protoc 1, 16-22.

Selkoe, D.J. (2001). Alzheimer's disease: genes, proteins, and therapy. Physiol Rev 81, 741-766.

Sternberger, L.A., Hardy, P.H., Jr., Cuculis, J.J., and Meyer, H.G. (1970). The unlabeled antibody enzyme method of immunohistochemistry: preparation and properties of soluble antigenantibody complex (horseradish peroxidase-antihorseradish peroxidase) and its use in identification of spirochetes. J Histochem Cytochem 18, 315-333.

Tan, J.M., Wong, E.S., Kirkpatrick, D.S., Pletnikova, O., Ko, H.S., Tay, S.P., Ho, M.W., Troncoso, J., Gygi, S.P., Lee, M.K., Dawson, V.L., Dawson, T.M., and Lim, K.L. (2008). Lysine 63-linked ubiquitination promotes the formation and autophagic clearance of protein inclusions associated with neurodegenerative diseases. Hum Mol Genet 17, 431-439.

Tucker, S.M., Borchelt, D.R., and Troncoso, J.C. (2008). Limited clearance of pre-existing amyloid plaques after intracerebral injection of Abeta antibodies in two mouse models of Alzheimer disease. Journal of Neuropathology and Experimental Neurology 67, 30-40.

Van Leeuwen, F.W., De Kleijn, D.P., Van Den Hurk, H.H., Neubauer, A., Sonnemans, M.A., Sluijs, J.A., Koycu, S., Ramdjielal, R.D., Salehi, A., Martens, G.J., Grosveld, F.G., Peter, J., Burbach, H., and Hol, E.M. (1998). Frameshift mutants of beta amyloid precursor protein and ubiquitin-B in Alzheimer's and Down patients. Science 279, 242-247.

Van Leeuwen, F.W., Van Tijn, P., Sonnemans, M.A., Hobo, B., Mann, D.M., Van Broeckhoven, C., Kumar-Singh, S., Cras, P., Leuba, G., Savioz, A., Maat-Schieman, M.L., Yamaguchi, H., Kros, J.M., Kamphorst, W., Hol, E.M., De Vos, R.A., and Fischer, D.F. (2006). Frameshift 
proteins in autosomal dominant forms of Alzheimer disease and other tauopathies. Neurology 66, S86-92.

Van Tijn, P., De Vrij, F.M., Schuurman, K.G., Dantuma, N.P., Fischer, D.F., Van Leeuwen, F.W., and Hol, E.M. (2007). Dose-dependent inhibition of proteasome activity by a mutant ubiquitin associated with neurodegenerative disease. Journal of Cell Science 120, 1615-1623.

Van Tijn, P., Hobo, B., Verhage, M.C., Oitzl, M.S., Van Leeuwen, F.W., and Fischer, D.F. (2011). Alzheimer-associated mutant ubiquitin impairs spatial reference memory. Physiol Behav 102, 193-200.

Vandesompele, J., De Preter, K., Pattyn, F., Poppe, B., Van Roy, N., De Paepe, A., and Speleman, F. (2002). Accurate normalization of real-time quantitative RT-PCR data by geometric averaging of multiple internal control genes. Genome Biol 3, RESEARCH0034.

Verhoef, L.G., Heinen, C., Selivanova, A., Halff, E.F., Salomons, F.A., and Dantuma, N.P. (2009). Minimal length requirement for proteasomal degradation of ubiquitin-dependent substrates. FASEB Journal 23, 123-133.

Warrington, J.A., Nair, A., Mahadevappa, M., and Tsyganskaya, M. (2000). Comparison of human adult and fetal expression and identification of 535 housekeeping/maintenance genes. Physiol Genomics 2, 143-147.

Xia, Z.P., Sun, L., Chen, X., Pineda, G., Jiang, X., Adhikari, A., Zeng, W., and Chen, Z.J. (2009). Direct activation of protein kinases by unanchored polyubiquitin chains. Nature 461, 114119.

Zhao, X., and Yang, J. (2010). Amyloid-beta peptide is a substrate of the human 20S proteasome. ACS Chem Neurosci 1, 655-660. 


\section{CHAPTER 6}

Paradoxical effects of mutant ubiquitin and $\mathrm{y}$-secretase during modelled Alzheimer's disease neuropathogenesis

R.J. Gentier, J.Stevens, L.M. Verheijen, C.D. van 't Hekke, D. van den Hove, D.H.J.P. Hermes, H.W.M. Steinbusch, J.J. Cheng, M.O. Grimm, V. J. Haupenthal, W. Annaert, T. Hartmann and F.W. van Leeuwen.

In preparation 


\section{Abstract}

$A \beta$ plaque formation is a prominent cellular hallmark of Alzheimer's disease (AD). To date, immunization and $\gamma$-secretase inhibitor trials in AD patients have not been effective in terms of curing or ameliorating dementia. In studies with a transgenic AD mouse model it was shown that there is limited clearance of pre-existing amyloid plaques when aging has started. Therefore, more knowledge about the mechanism and relevance of $A \beta$ plaque formation is required before reconsidering trials. We addressed this issue by crossbreeding two transgenic mouse lines (\#85; APPswe, PS1 1 exon 9, dubbed APPPS1) with a line (\#3413), suffering from loss of protein quality control by the ubiquitin proteasome system (UPS). Counterintuitively, the crossbreed (APPPS1/UBB ${ }^{+1}$ ) showed a striking decrease in $\mathrm{A} \beta$ plaque plaque load formation during aging by an unknown mechanism.

Here we show in APPPS1 mice that $\mathrm{Y}$-secretase activity was specifically decreased during early aging, whereas $\alpha$-and $\beta$-secretase activities were unaffected. Surprisingly, in the crossbreed the $\mathrm{Y}$-secretase activity was transiently enhanced at the age of 6 months; $a$ period where $A \beta$ plaque formation is attenuated. Our data implicate that there is a strong interaction between a dysfunctional UPS and $A \beta$ plaque formation being mediated specifically via $Y$-secretase and this is due to a flawed but transient PS1 expression in APPPS1 mice at 6 months of age that is absent in the crossbreed. Both APPPS1 mice and the crossbreed showed similar impairments in self-care, spatial memory (Morris water maze) and working memory (Y-maze) at 9 months of age whether or not they had reduced $A \beta$ plaque load, which illustrates the low of impact of $A \beta$ deposits on the behavioral phenotype.

In conclusion, the present results show an inverse correlation between $\mathrm{Y}$-secretase activities and $A \beta$ plaque load that will contribute to a better understanding of strategies to ameliorate or cure $A D$ via preservation of $y$-secretase modulation. 


\section{Introduction}

Alzheimer's disease (AD) currently affects at least 20 million people worldwide and its prevalence is expected to treble by 2050 (IGAP, 2015). Amyloid $\beta$ (Aß) plaques are a prominent cellular hallmark of $A D$ and is the foundation of the well-known 'amyloid hypothesis' (Glenner and Wong, 1984). According to this hypothesis $A \beta$ accumulation is the initiating event in the pathogenesis of $A D$ which is caused by a disrupted endoproteolytic cleavage of amyloid precursor protein (APP). The aberration in the cleavage can be due to mutations in the APP, presenilin 1 (PS1) and presenilin 2 (PS2) genes and, which results in different $A \beta$ species by favoring cleavage of APP by $\gamma$ - and $\beta$ secretase (Hardy and Selkoe, 2002). To date, immunization trials in AD patients have not been effective in terms of curing or ameliorating dementia. It has been suggested that these trials started apparently too late (Lannfelt et al., 2014). In studies with transgenic animals, it was indeed shown that there is limited clearance of pre-existing amyloid plaques (Tucker et al., 2008). In addition, treatments with $\gamma$-secretase inhibitors have so far been unsuccessful. Therefore, more knowledge about the mechanism and relevance of $A \beta$ plaque formation is required before reconsidering trials (De Strooper, 2014). In this essay it was suggested that trials using broad-spectrum $\mathrm{y}$-secretase inhibitors were prematurely halted upon unwarranted feelings. Further research on the mechanism of $A \beta$ plaque formation and the role of $\gamma$-secretase is thus required. In the present paper we have investigated another aspect of the puzzle related to $Y$-secretase and $A \beta$ plaque formation by focussing on proteasome dysfunction in vivo.

Recently, pooled Genome Wide Association Studies (GWAS) and pathway analysis identified protein ubiquitination as one of the prime targets for AD (IGAP, 2015). Earlier, a brain site specific strategy was used to compare the proteomes of prefrontal cortex, hippocampus and cerebellum in brains of $A D$ patients. An ingenuity pathway analysis identified 31 proteins which were significantly altered. These proteins had a strong interaction with ubiquitin $C$ (UBC) signaling pointing to a dysfunctional ubiquitin proteasome system (UPS) as a causative factor in AD (Manavalan et al., 2013).

Synaptic plasticity is affected in $A D$ and is a part of processes that involve metabolism of $5-8 \%$ of brain proteins each day (Dennissen et al., 2012). This turnover requires an efficient 
protein quality control (PQC) for which the UPS and autophagy are mainly responsible (Tsakiri and Trougakos, 2015). We discovered that the mistranscription of the ubiquitin $B$ (UBB) gene can result in accumulation of mutant ubiquitin $\mathrm{B}^{+1}\left(\mathrm{UBB}^{+1}\right)$ protein in the cellular hallmarks (plaques and tangles) of sporadic and autosomal dominant AD cases as well as other tauopathies, suggesting a pathological function (van Leeuwen et al., 1998;van Leeuwen et al., 2006;Dennissen et al., 2012). $\mathrm{UBB}^{+1}$ is also involved in polyglutamine diseases but not in synucleinopathies (Fischer et al., 2003;de Pril et al., 2004); UBB+1 compromises PQC by dose-dependently inhibiting the UPS (Dennissen et al., 2012) via inhibition of de-ubiquitinating enzymes (DUB) (Krutauz et al., 2014). We showed that UBB ${ }^{+1}$ is unable to ubiquitinate degradable targets and cannot be deubiquitinated, thereby inhibiting the UPS. These events result in dysfunction of ER-associated degradation (ERAD), stress of mitochondria, neuritic clogging and accumulation of basic amino acids at mitochondria as a decisive toxic event, all resulting in a phenotype of impaired contextual behavior (Fischer et al., 2009;Dennissen et al., 2012;Schipanski et al., 2014;Braun et al., 2015).

For more than a decade a relation between a dysfunctional UPS and A $\beta$ plaque formation has been surmised but never proven in detail (Hong et al., 2014). It is now possible to address this issue by crossbreeding 2 transgenic ( $\operatorname{tg}$ ) lines; line \#3413 with postnatal UBB ${ }^{+1}$ overexpression, proteasomal inhibition and a proteome partly compatible with AD (Fischer et al., 2009) and line \#85 (APPPS1) with A $\beta$ plaque formation starting at 4 months of age (Garcia-Alloza et al., 2006). Surprisingly, a significant decrease in $A \beta$ plaques and levels of soluble $A \beta_{42}$ is found in the crossbreed (APPPS1/UBB ${ }^{+1}$ ) compared with APPPS1 mice at 6 months of age (van Tijn et al., 2012). Therefore, our next aims are to examine the molecular mechanisms behind $A \beta$ plaque decrease in the crossbreed. Here we address the role of $\gamma$ - secretase, the underlying mechanism of the $A \beta$ plaque formation and the behavioral consequences in the crossbreed. 


\section{Materials and methods}

\subsection{Animals}

Transgenic mouse models (tg) used in the present study 1) $\mathrm{UBB}^{+1}$ tg mice ( $\mathrm{n}=11(3 \mathrm{M}) ; \mathrm{n}=$ 9 (6M); n=14 (9M)) (line \#3413; (Fischer et al., 2009)) expressing human $\mathrm{UBB}^{+1} \mathrm{cDNA}$ in the postnatal brain on a pure $\mathrm{C} 57 / \mathrm{Bl} 6$ background under control of a murine calmodulin kinase II alpha (CaMKIl $\alpha)$ promoter, 2) double tg APPswePS1 $1_{\triangle \text { exong }}$ mice ( $n=8$ (3M); $n=9$ (6M); $n=13$ (9M)) (line \#85; APPPS1, (Jankowsky et al., 2004)) carrying the Swedish mutation (K594M/N595L) in chimeric mouse/human APP695 and human PS1 with a deletion of exon 9 (Jankowsky et al., 2001), both under control of a mouse prion promotor. Line \#85 was back-crossed to C57/Bl6 for at least seven generations and, thus can also be assumed to be of pure C57/Bl6 background. 3) These two tg lines were crossbred to generate APPPS1/UBB ${ }^{+1}$ mice $(n=7$ (3M); $n=5(6 M) ; n=6(9 M)$ (van Tijn et al., 2012). Nontransgenic littermates were used as controls $(n=12(3 M) ; n=5(6 M) ; n=11(9 M)$ ). Transgenic lines were maintained on their respective genetic background by breeding homozygous mice to wild-type (WT) mice for more than 20 generations (Couch et al., 2013). The mice from each genotype were age-matched littermates. Genotyping was performed by polymerase chain reaction analysis of tail DNA.

APPPS1 mice and the crossbreed at 6 months of age can suffer from silent epileptic insults. Therefore, embryos of the APPPS1 line were revitalized and crossed again with $\mathrm{UBB}^{+1} \mathrm{tg}$ mice. These mice received normal tap water and reached the age of 9 months without suffering silent epileptic insults. Mice were kept under standard animal housing conditions: a 12/12 h light- dark cycle with food and acidified water (3 and 6 months) and normal water (9 months) ad libitum in specific pathogen free conditions, to ensure comparability among individuals. All animal experiments were performed conforming to national animal welfare law and under guidance of the animal welfare committee of Maastricht University. Mice of 3, 6 and 9 months of age used for secretase measurements and Western blotting were sacrificed by cervical dislocation ( $\mathrm{CO}_{2}$ drug was not used). Brains were isolated, washed in physiological saline solution $\left(0.9 \% \mathrm{NaCl} ; 4^{\circ} \mathrm{C}\right)$ and cut into left and right hemispheres to store them separately in a cryovial in liquid nitrogen until further processing. Brains used 
for immunohistochemistry were processed differently. After the behavioral study, mice were sacrificed at 3, 6 or 9 months of age. Mice were deeply anesthetized with sodium pentobarbital and were perfused transcardially with Tyrode solution $(2.68 \mathrm{mM} \mathrm{KCl}, 0.245$ $\mathrm{mM} \mathrm{MgCl} 2.6 \mathrm{H} 2 \mathrm{O}, 136 \mathrm{mM} \mathrm{NaCl}, 0.289 \mathrm{mM}$ NaH2PO4.H2O, $5 \mathrm{mM}$ glucose, $12 \mathrm{mM}$ $\mathrm{NaHCO} ; \mathrm{pH} 7.4$ ) aerated with carbogen followed by $0.1 \mathrm{M}$ phosphate buffer containing $4 \%$ paraformaldehyde $(\mathrm{pH}$ 7.4). Brains were removed and placed in fixative overnight on a rocking table $4^{\circ} \mathrm{C}$. Subsequently, they were stored in a $1 \%$ sodium azide (NaN3) PBS solution in a cold room at $4^{\circ} \mathrm{C}$ until further processing. Mice brains used for secretase activity measurements were only washed with Tyrode solution. An overview of experiments performed with the 4 different mouse types at 3 different ages is shown in Figure 1.

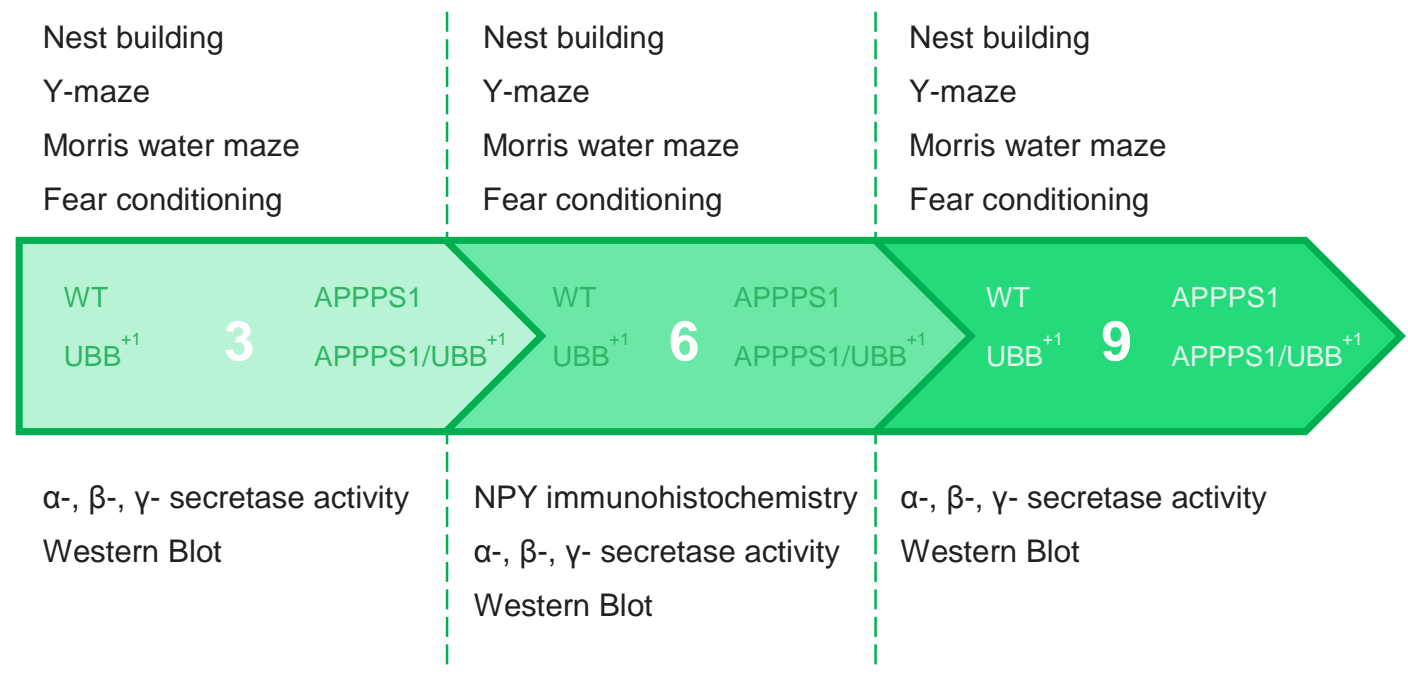

Figure 1: An overview of experiments performed with the WT mice and the 3 different tg mouse lines (UBB ${ }^{+1}, A P P P S 1, A P P P S 1 / U B B^{+1}$ ) at 3 age stages (3, 6 and 9 months). In the upper part a list of the behavioral experiments is shown in chronological order, in the lower part of the overview the experiments were performed after the mice were sacrificed.

\subsection{Immunohistochemistry}

To determine whether mice suffer from nonconvulsive epileptic insults during the behavioral examination, immunohistochemical analyses experiments were performed. Brains were isolated and processed as described before (Irmler et al., 2012). Free-floating 
brain sections of APPPS1, APPPS1/UBB ${ }^{+1}$ and control mice of 6 months old were incubated with a home-made antibody against Neuropeptide Y (Nypke (NPY); 1:1000, as a marker for epileptic insults in the hippocampus, (Minkeviciene et al., 2009) followed by incubation with biotinylated donkey anti-rabbit antibody (1:400) (Jackson Laboratories) and with avidin-biotin-peroxidase (ABC,1:400, Vector), both $1 \mathrm{~h}$ at $\mathrm{RT}$.

The staining was visualized with 3,3'-diaminobenzidine tetra hydrochloride (DAB) solution intensified by $0.2 \%$ nickel ammonium sulphate (pH7.6). The sections were mounted on gelatine-coated glass slides, air dried, dehydrated, and cover slipped using Pertex (Histolab). Brain sections of APPPS1 mice and the crossbreed were analysed semiquantitatively by light microscopy. The intensity of the NPY staining in the hippocampus and cerebral cortex was scored as 'negative' (-) or 'positive' with a range from 'weak (+), mild $(++)$ to strong positive' (+++ or +++$)$. Furthermore, the same protocol and scoring scale were used for a primary antibody against $\mathrm{UBB}^{+1}$ (Ubi3, 1:1000, bleeding 160998, for details see (Fischer et al., 2003; Fischer et al., 2009) to confirm the genotyping.

\subsection{Determination of secretase activities}

The activity of the secretases in half hemispheres were determined as described previously (Grimm et al., 2013) with slight modifications. Briefly, mice brains were homogenized using $1.4 \mathrm{~mm}$ ceramic precellys in a Minilys (Peqlab, Erlangen, Germany) for $30 \mathrm{sec}$ at maximum speed in sucrose buffer (10mM Tris-HCl pH7.4, 1mM EDTA, 200mM sucrose). Samples were adjusted to a concentration of $4 \mathrm{mg} / \mathrm{ml}$ of total protein using BCA-Assay according to Smith et al (Smith et al., 1985). Post-nuclear fractions were prepared by centrifuging samples at $900 \mathrm{~g}$ for $10 \mathrm{~min}$ at $4^{\circ} \mathrm{C}$. Fractions enriched in membranes were prepared by centrifugation at $55000 \mathrm{rpm}$ for $75 \mathrm{~min}$ at $4^{\circ} \mathrm{C}$ in an Optima MAX Ultracentrifuge with a TLA55 rotor (Beckmann Coulter, Krefeld, Germany). The pellet was solubilized using 0.5mm glass precellys in a Minilys (Peqlab, Erlangen, Germany) for 10 sec at minimum speed in sucrose buffer (10mM Tris-HCl pH7.4, 1mM EDTA, 200mM sucrose). For determination of a-secretase activity, samples were supplemented with $5 \mu \mathrm{M} \mathrm{ZnCl} 2$ and $5 \mu \mathrm{M} \mathrm{Mg} 2 \mathrm{Cl} 2$.

Either $\alpha$-, $\beta$ - or $\gamma$-secretase activities were measured continuously at $37^{\circ} \mathrm{C}$ in a black 96 well plate (BD, Franklin Lakes, NJ USA) by detection of the cleavage of a specific substrate 
in a Safire2 Fluorometer (Tecan, Germany) for $\beta$-secretase activity or in an Infinite M1000Pro (Tecan, Germany) for $\alpha$-secretase and $y$-secretase activity.

For the determination of $\mathrm{y}$-secretase activity $500 \mu \mathrm{g}$ of protein per $100 \mu \mathrm{l}$ was used. Kinetic were started by adding 10 $\mathrm{MM} \gamma$-secretase substrate (\#565764, Calbiochem, Darmstadt, Germany). $y$-secretase activity was measured with an excitation wavelength of $355 \mathrm{~nm}$ (bandwidth 10nm) and an emission wavelength of 440nm (bandwidth 10nm). Kinetic was carried out for 50 cycles with intervals of 180 s.

For $\alpha$-secretase the substrate \#II-565767 was used while for $\beta$-secretase, \#IV-565758 was used (both from Calbiochem, Darmstadt, Germany). For measurement of $\beta$-secretase activity, samples were diluted 1:1 in PBS, $\mathrm{pH} 4.5$ and $100 \mu \mathrm{l}$ of this dilution, corresponding to $250 \mu \mathrm{g}$ of total protein, was used. Then, $20 \mu \mathrm{M}$ of $\beta$-secretase substrate IV (Calbiochem, Darmstadt, Germany) was added. $\beta$-secretase activity was measured with an excitation wavelength of $345 \mathrm{~nm}$ (bandwidth $5 \mathrm{~nm}$ ) and an emission wavelength of $500 \mathrm{~nm}$ (bandwidth $5 \mathrm{~nm})$. Kinetic was carried out for 75 cycles with intervals of 80 s.

For determination of $\alpha$-secretase activity $100 \mu \mathrm{l}$ sample, corresponding to $100 \mu \mathrm{g}$ total protein, were measured. The $\alpha$-secretase substrate II was used (Calbiochem, Darmstadt, Germany) in a final concentration of $3 \mu \mathrm{M}$. Measurement parameters were adjusted to an excitation wavelength of $340 \mathrm{~nm}$ (bandwidth $10 \mathrm{~nm}$ ) and an emission wavelength of $490 \mathrm{~nm}$ (bandwidth 10nm). Kinetic was carried out for 70 cycles with intervals of $120 \mathrm{~s}$.

Specificity of the assays were monitored by using the following secretase inhibitors: $\alpha$ -

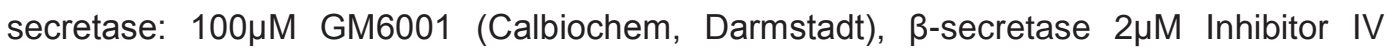

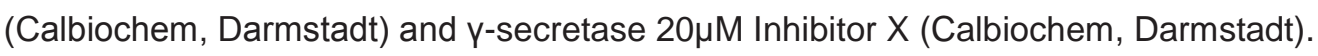

Statistical analyses were performed using GraphPad Prisma 6 Software (Grappa, San Diego, CA, USA) One-way analysis of variance (ANOVA), followed by Bonferroni's multiple comparisons test were used. Values are presented as mean \pm SEM. Differences between groups were considered significant when $p \leq 0.05$. 


\subsection{Quantitative Western Blotting}

The other brain hemispheres of these tg mouse lines were homogenized in lysis buffer consisting of STE buffer ( $250 \mathrm{mM}$ sucrose, $5 \mathrm{mM}$ Tris-HCl, and $1 \mathrm{mM}$ EGTA, pH 7.4) with $1 \%$ Triton X-100 and a mix of protease inhibitors (complete Roche, catnr: 11697498001) and phosphatase inhibitors $(2.5 \mathrm{mM}$ sodium pyrophosphate and $1 \mathrm{mM} \beta$-glycerol phosphate disodium salt) on ice for $20 \mathrm{~min}$. Lysates were centrifuged for $15 \mathrm{~min}$ at 13000 $\mathrm{rpm}$ at $4^{\circ} \mathrm{C}$. Total protein amounts of supernatants were quantified using a standard Bradford assay (Pierce). Absorbance ( $A=595 \mathrm{~nm} ; 0,1 \mathrm{~s} /$ well) was measured with a Victor ${ }^{3}$ reader and lysates were diluted in baxter water so that the values minus blanc were between 0.4 and 0.1 to be in the linear range of the standard curve. Samples (final protein concentration $=\mathrm{\gamma} 2 \mu \mathrm{g} / \mu \mathrm{l}$ ) were prepared with sample buffer (4x LDS and 4\% 2mercaptoethanol) and lysis buffer followed by incubation in a heater for $10 \mathrm{~min}$ at $70^{\circ} \mathrm{C}$. Of each sample, $20 \mu \mathrm{g}$ of protein was separated by electrophoresis (40 min. at $200 \mathrm{~V}$ and 400 $\mathrm{mA}$ ) in 1x MES running buffer (Invitrogen) on $4-12 \%$ bis-tris precasted gels (Invitrogen) along with See Blue Plus2 marker (Invitrogen). Proteins were transferred (100 V and 350 $\mathrm{mA}$ overnight at $4^{\circ} \mathrm{C}$ ) on nitrocellulose membranes (Invitrogen) using $1 \mathrm{x}$ transfer buffer (NUPAGE). Membranes were blocked using blocking buffer (5\% milk powder, TBS, 0.1\% Tween20) for 1 hour at room temperature (RT). Primary antibodies (3h at RT) were diluted in blocking buffer and were directed against nicastrin, human PS1 full-length (PS1-FL) and PS1 N-terminal fragment (PS1-NTF; APP full-length (APP-FL) and APP C-terminal fragment (APP-CTF); N-cadherin; synoviolin/HMG-CoA reductase degradation1 (HRD1); retention in endoplasmic reticulum sorting receptor 1 (RER1) and glyceraldehyde 3phosphate dehydrogenase (GAPDH) (see Table 1 for details). Primary antibodies were recognized by horseradish peroxidase-conjugated secondary antibodies (goat anti-mouse (GAMPO; Alexa 700, red) or goat anti-rabbit (GARPO; Alexa 800, green); 1:10000; $1 \mathrm{~h}$ at $\mathrm{RT})$. Blots were developed using enhanced chemiluminescence (ECL) Western Blotting kit (Perkin Elmer) and measured with Fuji LAS3000. Protein bands were quantified by measuring pixel intensity using AIDA Image Analyzer v4.27 software (Raytest) and normalized for GAPDH. Relative expressions were determined by calculating the ratio between WT mouse line and UBB ${ }^{+1}$, APPPS1 and mouse lines and their crossbreed within each corresponding blot and age group. 
Statistical analyses were performed using Mann-Whitney U-test. Values are presented as mean $\pm S E M$. Differences between groups were considered significant when $p \leq 0.05$. 


\begin{tabular}{|c|c|c|c|c|c|c|c|c|c|}
\hline ฏ & 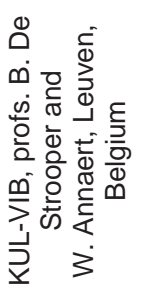 & $\begin{array}{l}\text { E్ } \\
\text { ర్ } \\
\frac{0}{<}\end{array}$ & 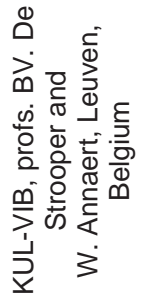 & 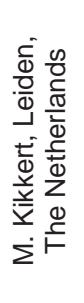 & 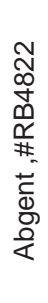 & 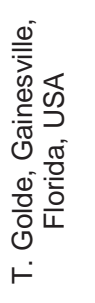 & 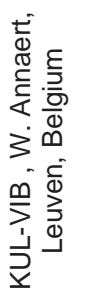 & 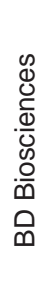 & 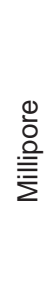 \\
\hline 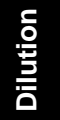 & 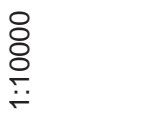 & 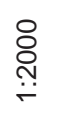 & 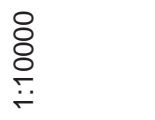 & 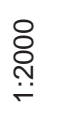 & $\begin{array}{l}8 \\
\stackrel{8}{\circ} \\
\stackrel{-}{-}\end{array}$ & $\begin{array}{l}8 \\
\stackrel{8}{\circ} \\
\stackrel{-}{-} \\
-1\end{array}$ & $\begin{array}{l}8 \\
\stackrel{8}{+} \\
\stackrel{-}{-}\end{array}$ & $\begin{array}{l}8 \\
\text { ठ } \\
\stackrel{-}{-} \\
\end{array}$ & 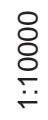 \\
\hline 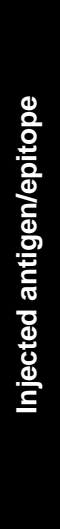 & 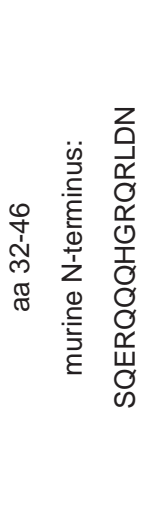 & 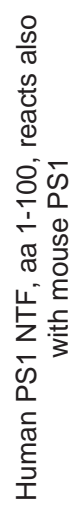 & 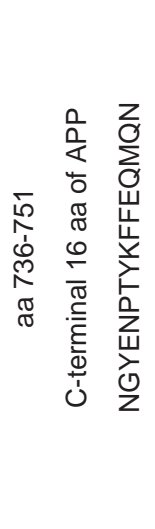 & 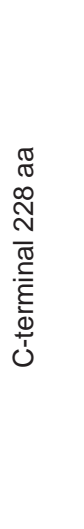 & 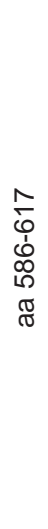 & 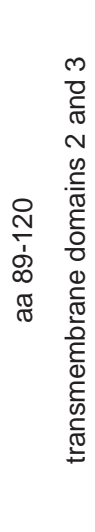 & 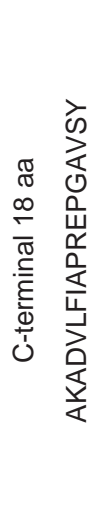 & 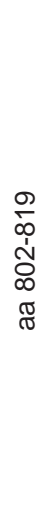 & 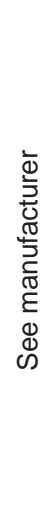 \\
\hline $\begin{array}{l}\frac{y}{d} \\
\frac{\delta}{0} \\
\dot{0}\end{array}$ & 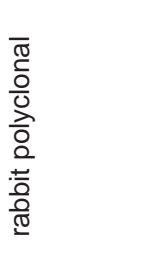 & 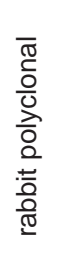 & 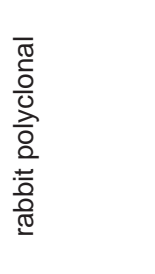 & 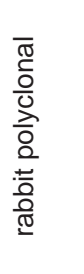 & 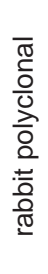 & $\begin{array}{l}\overline{0} \\
\frac{0}{0} \\
\frac{0}{0} \\
\text { 응 } \\
\frac{0}{0} \\
\text { 은 }\end{array}$ & 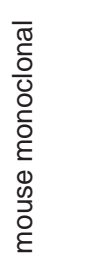 & 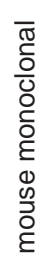 & 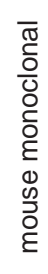 \\
\hline 용 & $\stackrel{9}{\vec{D}}$ & 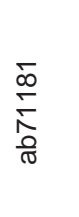 & ஜ్ర & 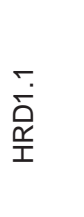 & $\begin{array}{l}\sum_{z}^{-1} \\
\text { co } \\
\text { i } \\
\dot{0} \\
\text { 辛 }\end{array}$ & 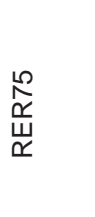 & ర్ర & 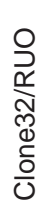 & 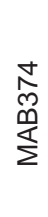 \\
\hline $\begin{array}{l}\text { 중 } \\
8 \\
\frac{8}{\mathrm{Z}} \\
\frac{\mathrm{z}}{4}\end{array}$ & 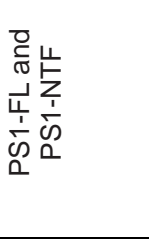 & 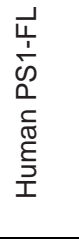 & 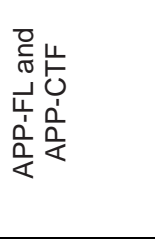 & $\begin{array}{l}\stackrel{-1}{Q} \\
\text { 足 }\end{array}$ & 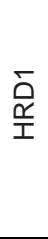 & $\begin{array}{l}\vec{r} \\
\vec{w} \\
\widetilde{\sim}\end{array}$ & 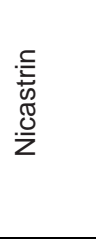 & $\begin{array}{l}\frac{5}{\bar{d}} \\
\frac{1}{\bar{T}} \\
\frac{0}{0} \\
\dot{1}\end{array}$ & 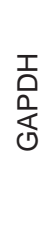 \\
\hline
\end{tabular}




\subsection{Behavioral experiments}

\subsubsection{Nest building}

The mice were housed in single cages containing sawdust for 1 week. On the day before testing, one piece of cotton ( $5 \times 5 \mathrm{~cm}$, Nestlets; Ancare, Bellmore) was introduced in the home cage to permit nesting. The following morning the nests were assessed, according to a five-point scale ranging from 1 to 5 (Deacon et al., 2008) as follows: $1=$ nestled not noticeably touched, $2=$ nestled partially torn up, $3=$ mostly shredded but often with no identifiable nest site, $4=$ an identifiable, but flat nest, and $5=a$ (near) perfect nest.

\subsubsection{Spontaneous alternation $\mathrm{Y}$-maze test}

Spontaneous alternation performance was tested using a symmetrical Y-maze. Each mouse was placed in the end of one arm of the Y-maze and was allowed to explore freely through the maze during a 6 min session. The sequence and total number of arms entered were recorded. Percentage alternation is the number of triads containing entries into all three arms divided by the maximum possible alternations (the total number of arms entered minus 2) $X 100$. The total distance moved $(\mathrm{cm})$ during this test was recorded as well.

\subsubsection{Morris water maze}

The Morris water maze (MWM) was a white circular pool (80 $\mathrm{cm}$ in diameter) divided into four equal imaginary quadrants for data analysis. The water was made opaque by addition of white non-toxic latex paint, water temperature was maintained at $24 \pm 2 \mathrm{C}^{\circ} .0 .5 \mathrm{~cm}$ beneath the surface of the water and hidden from mouse view was a white circular platform $6 \mathrm{~cm}$ in diameter. The swimming patterns of the mice were recorded with a video camera mounted above the center of the pool and analyzed with Ethovision video-tracking system. The water maze was located in a room with several visual stimuli hanging on the walls to provide spatial cues. The acquisition phase was carried out during four to five consecutive days. On each training day/session, the mice received 3 consecutive training trials with the hidden platform kept in a constant position. A different starting location was used for each trial. Mice, that were not able to find the platform within 60 s, were guided to the platform by 
the experimenter. To assess spatial retention, a 60s probe trial with the platform removed from the pool was given one day after the last hidden platform trial. The time spent swimming in the quadrants was recorded, and the percentages of time spent in the target quadrant (where the platform was located during hidden platform training) were analyzed.

\subsubsection{Statistical analyses}

Comparisons between groups for the behavioral measures were done by a one-way ANOVA or two-way ANOVA with repeated measures, and post-hoc LSD or Bonferroni's $t$ tests were performed when appropriate. Paired t-test was used to compare the percentage time spent between the target quadrant and the opposite quadrant within each group in the probe trial of water maze, and between the percentage freezing of baseline and context fear conditioning within each group in fear conditioning experiments.

\section{Results}

In the present study, the mechanism of the effects of decreased $A \beta$ plaque and $A \beta_{42}$ levels in APPPS1/UBB ${ }^{+1}$ mice compared to APPPS1 mice at 6 months of age was investigated. This was achieved by secretase activity measurements and Western blot analyses. Whether a decreased $A \beta$ plaque load has behavioral consequences was also examined in the present study.

\subsection{Immunohistochemistry}

To confirm whether changes in behavior in APPPS1 and the crossbreed mice were not due to silent epileptic insults, immunohistochemistry in 6-month-old mice was performed for NPY after behavioural testing. In both mutant mouse (APPPS1 and APPPS1/UBB ${ }^{+1}$ ) lines and in WT mice positive immunoreactivity of NPY was found in the hippocampus and cerebral cortex. No obvious differences in the amount of stained neuronal cells or intensity of NPY staining among mouse lines were noted. These data strongly suggest that these mice did not suffer from any (silent) epileptic insults at the age of 6 months during the behavioral observations were made. 
Immunohistochemistry with the Ubi3 antibody confirmed the genotyping, namely that all mice from the crossbreed and line 3413 carried the $\mathrm{UBB}^{+1}$ mutation, This was shown by the presence of $\mathrm{UBB}^{+1}$-stained neuronal granular cells of the dentate gyrus (DG) and of pyramidal cells in Cornu Ammonis (CA) 1-3 of the hippocampus and layers of the cerebral cortex.

\subsection{Secretase activity and Western blot experiments}

The activity levels for $\alpha$ - and the $\beta$-secretase were unaffected in the 4 different mouse lines at 3, 6 and 9 months (Figure 2). In contrast, the activity of the $y$-secretase enzyme was significantly decreased in the APPPS1 mice compared with WT mice ( 3 and 6 months $(p \leq 0.001), 9$ months $(p \leq 0.05))$ and compared with $\mathrm{UBB}^{+1}$ mice $(3$ months $(p \leq 0.01)$ and 6 months $(p \leq 0.001))$ mice (Figure 2$)$. The activity of the $y$-secretase enzyme was also significantly decreased in the crossbreed compared with WT mice ( 3 months $(p \leq 0.01), 6$ and 9 months $(p \leq 0.05))$. However, at 6 months of age, the $\gamma$-secretase activity was significantly increased in the crossbreed compared with APPPS1 mice (Figure 2). 


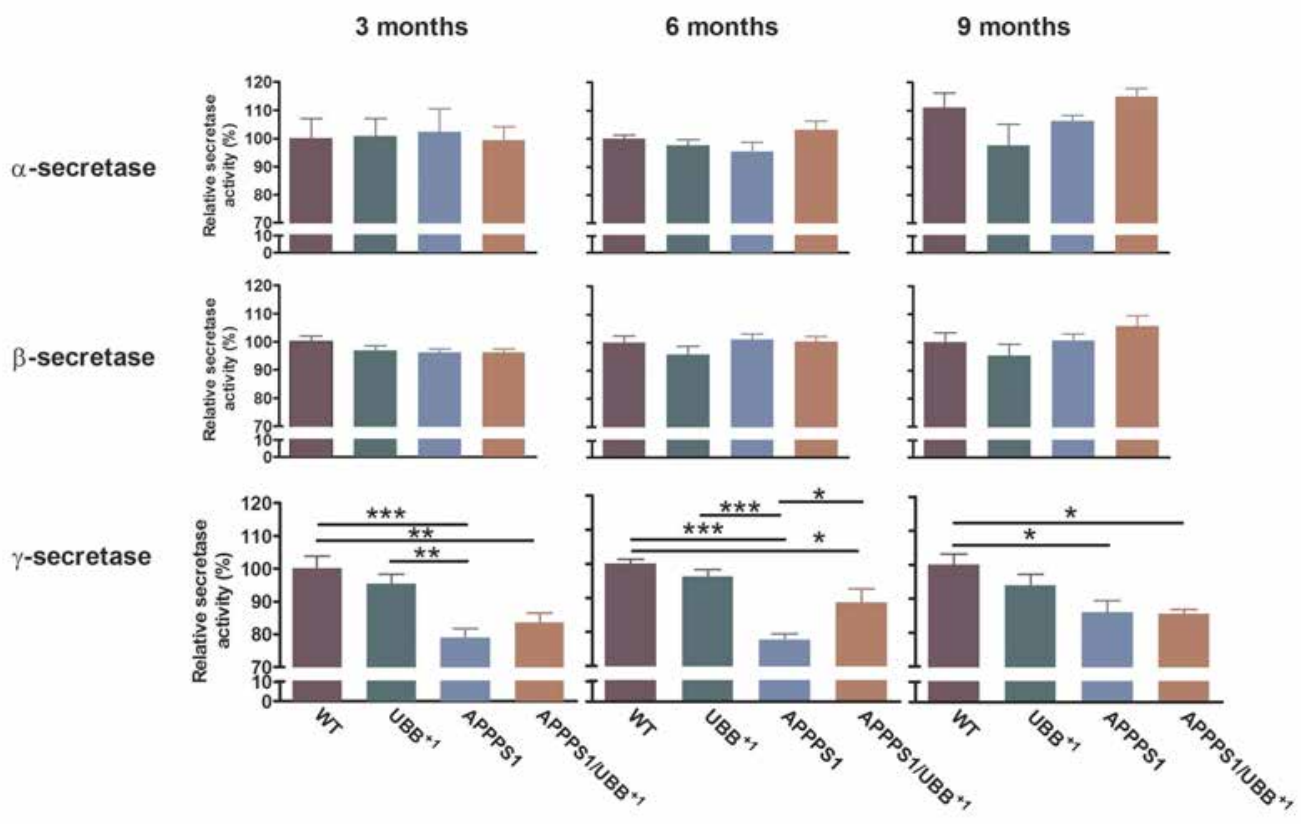

Figure 2: Levels of $\alpha$-, $\beta$-, and $\gamma$-secretase activity in the 4 different mouse lines. $\alpha$ - and $\beta$-secretase activities were unaffected. In the APPPS1 and the crossbreed; $\gamma$-secretase activity was decreased significantly at 3, 6 and 9 months of age compared with $W T$ and $U B B^{+1}$. However, in the crossbreed, the $\gamma$-secretase activity was enhanced at the age of 6 months compared with the double APPPS1 tg mouse line 85. ${ }^{*} p \leq 0.05 ;{ }^{* *} p \leq 0.01 ;{ }^{* * *} p \leq 0.001$.

Different components of the multi-subunit $\gamma$-secretase complex and substrates were examined in Western blot experiments: PS1 and APP (Figure 3). The PS1-NTF levels of the crossbreed were significantly $(p \leq 0.001)$ increased compared with APPS1 mice at 6 months of age. No changes were found in the comparison between APPPS1 and APPPS1/UBB ${ }^{+1}$ mice for PS1-NTF at 3 and 9 months of age (Figure 3). In keeping with the PS1-NTF differences between the two tg lines a significantly decreased $\beta$-APP-CTF level was shown in the APPS1/UBB ${ }^{+1}$ crossbreed compared with APPPS1 mice at the age of 6 months. No significant differences were found in $\beta$-APP-CTF between the two lines at 3 and 9 months of age. In the crossbreed the levels of APP-CTF and APP-CTF/FL ratio were similar to the levels of APPPS1 mice at 9 months of age. 
A

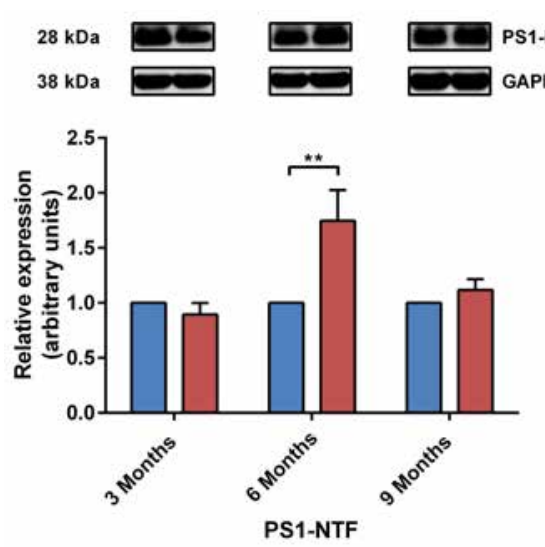

B

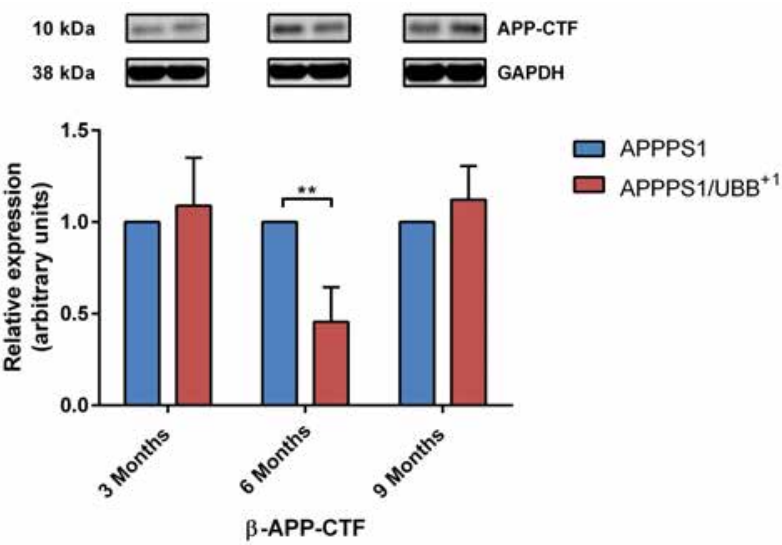

Figure 3: Expression levels of the PS1-NTF and $\beta$-APP-CTF measured by Western blot. A significantly increased $\left({ }^{* *} p \leq 0.01\right)$ PS1-NTF level and a significantly decreased $\left({ }^{* *} p \leq 0.01\right)$ APP-CTF level in APPPS1/UBB ${ }^{+1}$ mice compared to APPPS1 mice at 6 months of age was observed.

In addition, nicastrin, a $\mathrm{Y}$-secretase-substrate receptor, as well as N-cadherin (FL and CTF), a substrate for PS1 cleavage, were measured (data not shown). RER1, a small (196 aa) hydrophic protein, is the negative regulator of the stepwise assembly of the $\mathrm{Y}$-secretase assembly during ER-to-Golgi transport. Linked to RER1 is synoviolin, an E3 ubiquitin ligase implicated in ER-associated degradation and degradation of immature nicastrin and RER1. The expression levels of both proteins were also examined via Western blot. These measurements were performed as well for the 3 age stages in the 4 different mouse lines. For each of these proteins, no significant changes were found in the expression levels among the 4 lines at each age stage (see General Discussion).

To determine if the $\mathrm{y}$-secretase measurements matches with the results of the Western blots we have plotted the $\mathrm{Y}$-secretase activity against the PS1-NTF levels of the 4 different lines (4 different colors (purple $=$ WT, green $=\mathrm{UBB}^{+1}$, blue $=$ APPPS1 and $\mathrm{red}=$ crossbreed) at different ages which are represented by different dot sizes (small=3M, medium $=6 \mathrm{M}$ and large $=9$ months). A moderate positive correlation was present between $\mathrm{Y}$-secretase activity and PS1-NTF as shown in Figure 4. 


\section{Correlation PS1-NTF}

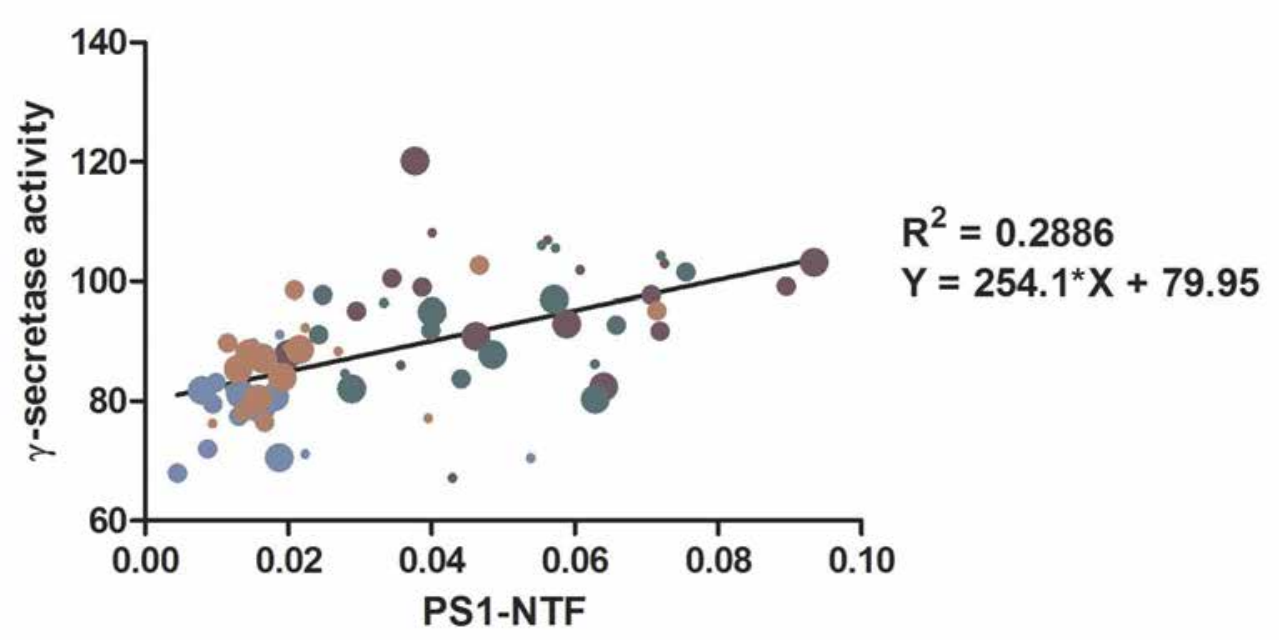

Figure 4: Correlation between $\gamma$-secretase activity and PS1-NTF with purple $=W T$, green $=U B B^{+1}$, blue $=$ APPPS1 and red $=A P P P S 1 / U B B^{+1}$. The different dot sizes represent the 3 different ages (small $=3 \mathrm{M}$, medium $=6 \mathrm{M}$ and large $=9$ months)

\subsection{Behavioral experiments: 3 and 6 months}

The behavioral analyses performed with mice (WT, UBB ${ }^{+1}$, APPPS1 and APPPS1/UBB ${ }^{+1}$ ) aged 3 and 6 months, revealed there were no cognitive deficits in the 3-month-old mice. However, at the age of 6 months hippocampus dependent associative learning and memory as assessed by Morris water maze were impaired in UBB ${ }^{+1}$, APPPS1 and APPPS1/UBB ${ }^{+1}$ transgenic mice. APPPS1/UBB ${ }^{+1} \mathrm{tg}$ lines showed similar impairments in self-care, spatial learning, and reference memory, whether or not they had reduced $A \beta$ deposits.

Subsequently sections from the 6-month-old mice were immunocytochemically stained for the presence of NPY. Analysis of the 4 mouse lines tested did not show a clear difference in NPY staining indicating that the behavioral testing was performed without interference from epilepsy (data not shown).

If the APPPS1 and the crossbreed with $\mathrm{UBB}^{+1}$ are aging more than 6 months almost half of the populations suffer from epileptic insults. To decrease the incidence of epilepsy we 
back crossed the APPPS1 mice. However the epileptic insults still occurred. Also adding gel blocks soaked with normal drinking water did not result in an improvement. Therefore we decided to revitalize frozen embryos of the APPPS1 mice and kept them on normal tap water (previously the water was acidified, $\mathrm{pH}=2.5$ ). Up till now no clear signs of epilepsy were found in mice older than 6 months. So we have started an experiment to measure the behavioral read outs at 9 months of age. At this age there is a full blown $A ß$ plaque deposition (van Tijn et al., 2012)

\subsection{Behavioral experiments: 9 months}

After revitalization, the male transgenic mice aged 9 months were tested in several behavioral screens: nestbuilding, Y-maze and the Morris water maze. The results of these tests are shown in Figure 5.

\subsubsection{Nestbuilding}

The APPPS1/UBB ${ }^{+1} \operatorname{tg}$ mice obtained significantly lower nesting score compared with the $W T(p \leq 0.05)$ and the UBB ${ }^{+1}$ mice $(p \leq 0.01)$. The APPPS1 mice had a nesting score in the same range as the WT and the $\mathrm{UBB}^{+1}$ mice (Figure 5).

\subsubsection{Y-maze}

The successful $\%$ alternations were measured after $4,6,8 \ldots$ up to 48 alternations for each of the 4 different mouse lines (Figure 5). The APPPS1/UBB ${ }^{+1}$ mice were very anxious in the beginning of the experiment which is reflected in a low $y$-maze alternation score. For example after 12 alternations (Y12), the APPPS1/UBB ${ }^{+1}$ mice showed a significantly decreased number of successful \% alternations compared with $\mathrm{WT}$ and $\mathrm{UBB}^{+1}$ mice $(p \leq 0.05)$. The successful \% alternations increased for these tg mice during the experiment to some higher $\%$, however even at $\mathrm{Y} 48$, the APPPS1/UBB ${ }^{+1}$ mice showed a significantly lower successful \% alternation score $(p \leq 0.01)$ compared to $W T$ and $\mathrm{UBB}^{+1}$ mice. The APPPS1 mice showed in the beginning of the $Y$-maze experiment also less successful alternations compared with the WT mice at $Y 12(p \leq 0.05)$. These mice increased further their successful alternations during the experiment and at $Y 48$ there were no significant 
differences anymore in working memory between these $\operatorname{tg}$ mice and the WT mice. The levels of successful alternations were always higher in the APPPS1 mice compared with the APPPS1/UBB ${ }^{+1}$ mice.

During the $\mathrm{Y}$-maze experiment, also the distance moved $(\mathrm{cm})$ was measured for each of the 4 mouse lines. APPPS1 and APPPS1/UBB ${ }^{+1}$ mice moved a significantly longer distance in the $Y$-maze compared to WT $(p \leq 0.001)$ and $\mathrm{UBB}^{+1}(p \leq 0.05$ compared with APPPS1 and $\mathrm{p} \leq 0.01$ compared with APPPS1/UBB ${ }^{+1}$ ) mice.

\subsubsection{Morris water maze}

In the MWM experiment the APPPS1 mice and the APPPS1/UBB ${ }^{+1}$ mice needed significantly more time to find the platform in the white circular pool compared to WT mice at day 5 of the acquisition phase (Figure 5). The repeated measures analysis showed for the MWM that the APPPS1 gene causes a worse performance compared to the UBB ${ }^{+1}(p$ $\leq 0.05)$ and the interaction between APPPS1xUBB ${ }^{+1}(p \leq 0.05)$. This means that the APPPS1 gene is responsible for the fact that mice with this gene need significantly more time to find the platform. During the probe trial, the time spent swimming in the quadrants was recorded and the percentages of time spent in each of the quadrants were calculated. APPPS1 ( $p \leq 0.05$ ) and APPPS1/UBB ${ }^{+1}$ mice spent significantly $(p \leq 0.01$ ) less time in the target quadrant compared to WT mice (Figure 5). Neither tg mice lines reached above the $25 \%$ chance level and the Post-hoc LSD measurement demonstrated that this is because they both spend significantly ( $p \leq 0.05)$ more time in the opposite quadrant compared to the WT mice. 

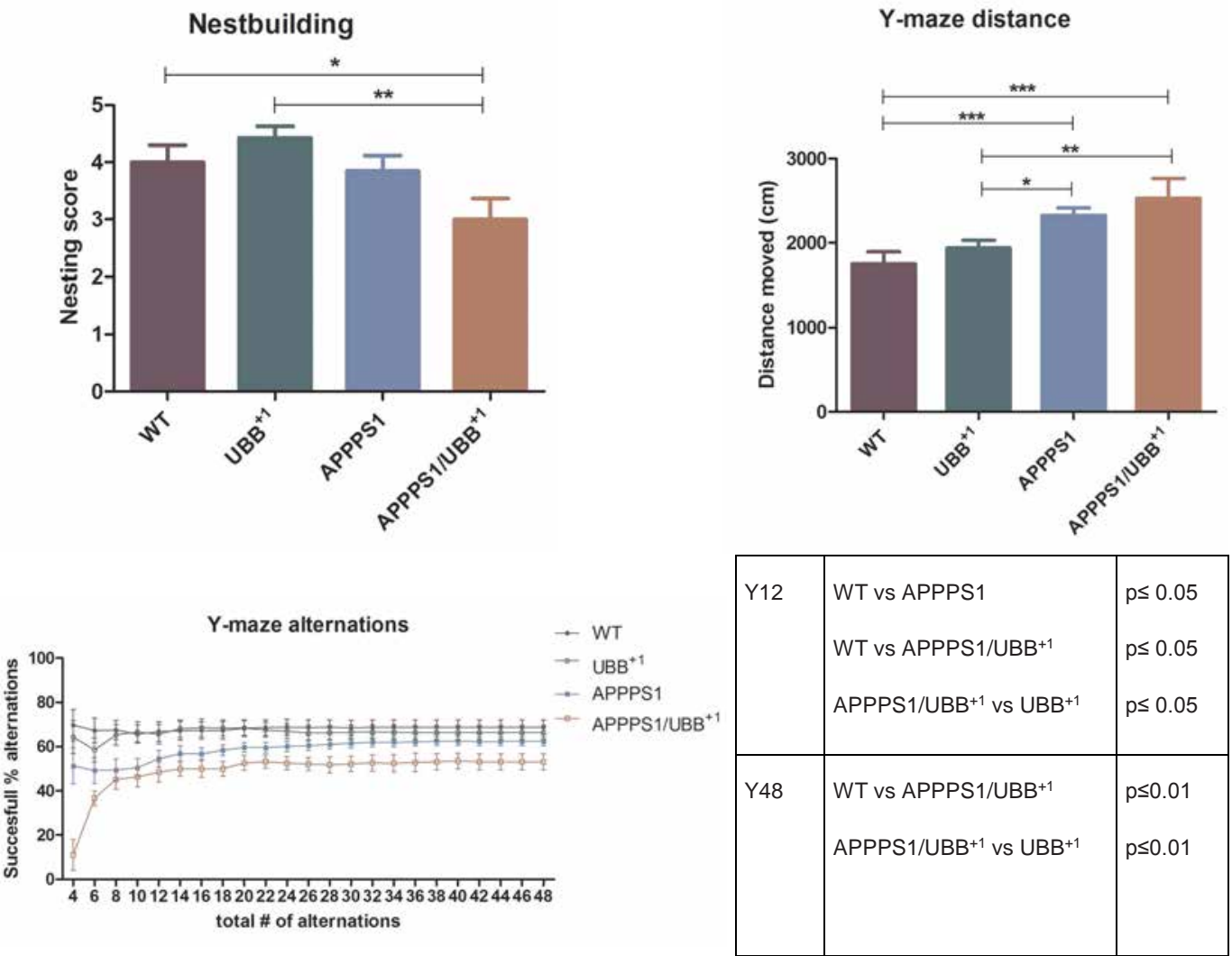

$\rightarrow$ WT

$\rightarrow \mathrm{UBB}^{+1}$

- APPPS1

APPPS1/UBB ${ }^{+1}$

MWM escape latency

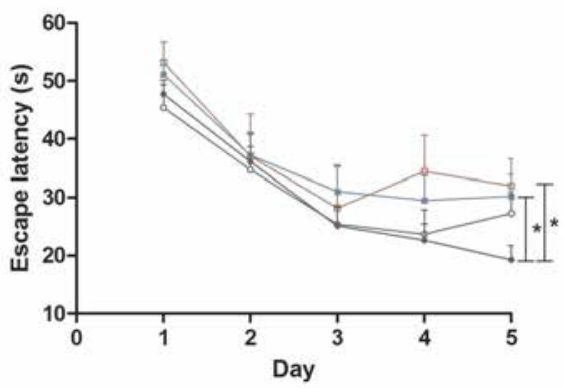

$\rightarrow \mathrm{WT}$

$\rightarrow \mathrm{UBB}^{+1}$

$\rightarrow$ APPPS 1

- APPPS1/UBB $^{+1}$

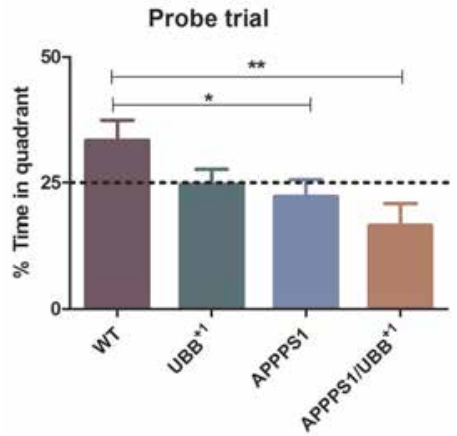

Figure 5: Nestbuilding, Y-maze and MWM measurements examined in 9-month-old male mice with different genotypes: WT, UBB ${ }^{+1}, A P P P S 1$ and APPPS1/UBB ${ }^{+1} ;{ }^{*} p \leq 0.05 ;{ }^{* *} p \leq 0.01 ;{ }^{* * *} p \leq 0.001$ 


\section{Discussion}

UPS dysfunction has been shown to be associated for a long time with AD pathogenesis (Mori et al., 1987; Perry et al., 1989) and is considered as a late event (Layfield et al., 2003). Misframed ubiquitin $\left(\mathrm{UBB}^{+1}\right)$ was localized in neurofibrillary tangles, neuropil threads and in the dystrophic neurites of plaques in aged non-demented controls, suggesting UPS dysfunction in earlier stages of AD (van Leeuwen et al., 1998;van Leeuwen et al., 2000). Since then substantial experimental data have appeared showing that $\mathrm{UBB}^{+1}$ indeed inhibits the proteasome in a dose-dependent way (van Tijn et al., 2007). Recently, strong support for a central role of the UPS in AD was presented by GWAS and proteomic studies (Manavalan et al., 2013;IGAP, 2015), culminating in the view that the UPS is a prime target for $A D$ therapeutics. The link to $A \beta$ pathology remains obscure because it was thought that the enzymatic processing of the amyloid precursor protein was spatially separated from cytosolic actions of ubiquitin (Saido and Leissring, 2012). However, in the past decade a fair numbers of papers have appeared showing an interaction between the UPS and A $\beta$ pathogenesis (van Tijn et al., 2012; Hong et al., 2014)

In the present study the mechanistic relation between the UPS and $A \beta$ plaque formation in vivo has been worked out in detail using a novel crossbreed (APPPS1/UBB ${ }^{+1}$ ) in which $A \beta$ plaque formation is studied in an environment of proteasomal stress. We studied an age series ( 3,6 and 9 months) of this crossbreed during which $A \beta$ formation takes place and which can still be manipulated (Tucker et al., 2008). This time frame is essential as immunization procedures to diminish $A \beta$ plaque load in $A D$ patients started in a late phase of the disease and were not effective in terms of improvement of dementia (Holmes et al., 2008). We used the well documented transgenic mouse line \#85 (APPPS1) (Jankowsky et al., 2004), in a pure C57BL/6J genetic background which is relevant for behavioral readouts. (Couch et al., 2013). This mouse model is ideal because by the age of 4 weeks the UPS appeared to be inhibited (Liu et al., 2014), which is long before A $\beta$ plaque formation starts (Garcia-Alloza et al., 2006). By crossbreeding of lines \#85 and \#3413 with further postnatal inhibition of the UPS, an optimal new line was generated for the present study. 
We have already reported in this mouse model a significant decrease in $A \beta$ plaque load formation by immunohistochemistry supported by ELISA data showing that $A \beta_{42}$ levels diminish (van Tijn et al., 2012). This unexpected finding triggered the question about the mechanism of this phenomenon as well as the behavioral consequences.

\subsection{Immunohistochemistry}

$A D$ is associated with an increased risk of unprovoked seizures (Vossel et al., 2013). In tg mice overexpressing mutant APP that produce excessive amounts of $A \beta$, sporadic deaths of unknown origin have been reported, even at remarkably young ages (Hsiao et al., 1995). Sudden deaths in these mice may occur at any age but peak around 3-4 months when first plaques appear in the cortex and the hippocampus (Garcia-Alloza et al., 2006; Shemer et al., 2006). It has also been reported that the expression of NPY in brain regions is elevated after (prolonged) epileptic insults (Colmers and El Bahh, 2003;Minkeviciene et al., 2009). Since Minkeviciene et al. have shown that very strong NPY immunoreactivity in mossy fibers extending to the CA3 of the hippocampus was present in only APP transgenic mice with silent seizures, it can be concluded that a low level of NPY immunoreactivity is associated with an absence of epileptic insults (Minkeviciene et al., 2009). When we compared the NPY immunoreactivity of our sections to the staining illustrated in the study of Minkeviciene et al., we found a much lower expression of NPY in the hippocampal region in APPPS1, APPPS1/UBB ${ }^{+1}$ and WT mice. It is therefore very likely to exclude any epileptic insults or seizures from being a confounder in the behavioral changes observed.

\subsection{Secretase activity and Western blot experiments}

Remarkably, the three secretase activities ( $\alpha, \beta$ and $\gamma$ ) of the double tg line were never measured before. Whereas the activity of $\alpha$ - and $\beta$-secretase were unaffected (from 3 to 9 months of age), the activity of $\gamma$-secretase was diminished significantly in the double tg mice whereas in the crossbreed at 6 months of age the activity of this multimeric complex was significantly raised. The latter is in striking parallel with the immunohistochemical data (van Tijn et al., 2012). Subsequent Western blot analyses showed that the affected $Y$ secretase activity was due to changes in PS1-NTF expression. At 6 months of age the PS1-NTF levels were significantly increased in the crossbreed compared to the APPPS1 
mice. The $\beta$-APP-CTF expression at 6 months of age indeed mirrored the PS1-NTF expression. The PS1-enzyme forms together with PS2 the catalytic core of $\mathrm{Y}$-secretase and is responsible for the cleavage of type 1 transmembrane proteins such as APP, notch and cadherins. It undergoes proteolytic cleavage itself as well to form PS1-NTF and PS1-CTF which associates with nicastrin, anterior pharynx-defective 1 (Aph-1) and presenilin enhancer 2 (Pen2) to form the multisubunit $Y$-secretase complex. In the amyloidogenic pathway, $A \beta$ is produced from APP by sequential proteolytic cleavage of $\beta$ - and $\gamma$ secretase. After $\beta$-secretase has cleaved APP at the $\mathrm{N}$-terminus, the ectodomain of APP (APPs $\beta$ ) sheds off and results in APP-CTF. After cleavage of PS1, the PS1-NTF/PS1-CTF heterodimer cleaves APP-CTF and produces many isoforms of $A \beta$ including $A \beta_{40}$ and $A \beta_{42}$, of which the latter is more aggregate-prone (Spasic and Annaert, 2008;De Strooper et al., 2012).

In all 3 age groups, the levels of PS1-NTF were significantly decreased in APPPS1 and mice and the crossbreed compared to WT and $\mathrm{UBB}^{+1}$ mice (see General Discussion). This is consistent with another study which showed that the PS1 $1_{\triangle \mathrm{E} 9}$ mutation lacks the PS1 endoproteolysis site and prevents PS1 cleavage into NTF and CTF in differentiated, purified neurons (Woodruff et al., 2013). The double tg mice and the crossbreed both contain this mutation and therefore we could confirm the decrease in PS1-NTF levels in both mouse lines. As mentioned above, production of active PS1-NTF is crucial for proper $\mathrm{Y}$-secretase activity and further processing of APP-CTF to produce A $\beta$ peptides (Haass et al., 2012). In the present study, the $\beta$-APP-CTF was significantly increased in APPPS1 $(p \leq 0.001)$ and in the crossbreeding ( $p \leq 0.001)$ mice compared to UBB ${ }^{+1}$ and WT mice (see General Discussion).

Most importantly, PS1-NTF level was significantly increased in APPPS1/UBB ${ }^{+1}$ mouse line compared to APPPS1 mouse line at the critical age of 6 months. The onset of A $\beta$ pathology in the transgenic mouse model occurs after 4 months (Garcia-Alloza et al., 2006). This increase in PS1-NTF level can be caused by the fact that $\mathrm{UBB}^{+1}$ expression will inhibit the proteasome in the crossbreed.

It has been shown that PS1 is degraded by the 26S proteasome (Fraser et al., 1998;Van Gassen et al., 1999). Therefore, inhibition of proteasomal degradation of PS1, could lead 
to enhanced PS1-WT levels. PS1-WT is still able to be processed into NTF and CTF. Higher PS1-NTF levels mean that $\mathrm{Y}$-secretase activity is enhanced and more APP-CTF is cleaved in the crossbreed mice compared to APPPS1.

Alternatively, APPPS1 mice do not have $\mathrm{UBB}^{+1}$ induced proteasomal inhibition. Therefore, PS1-WT will be degraded by the proteasome and less PS1-WT will be processed, resulting in less PS1-NTF. Consequently, $y$-secretase activity will be lower and APP-CTF level will increase in APPPS1 mice compared to APPPS1/UBB ${ }^{+1}$ mice. These events are also in agreement with earlier published results (van Tijn et al., 2012) and with Figure 2. Counterintuitively, Woodruff et al. also suggested that less $\gamma$-secretase activity does not result in less $A \beta$ generation per se (Woodruff et al., 2013). Mutant $P S 1_{\triangle E 9}$ is able to substitute PS1 NTF/CTF heterodimer in the secretory complex, which generally increases the $A \beta_{42} / A \beta_{40}$ ratio (Figure 6). In this view, a link can be made between increased plaque load in APPPS1 mice (van Tijn et al., 2012), reduced $\mathrm{y}$-secretase activity (Figure 2) and increased $A \beta_{42}$ proportion (van Tijn et al., 2012). 


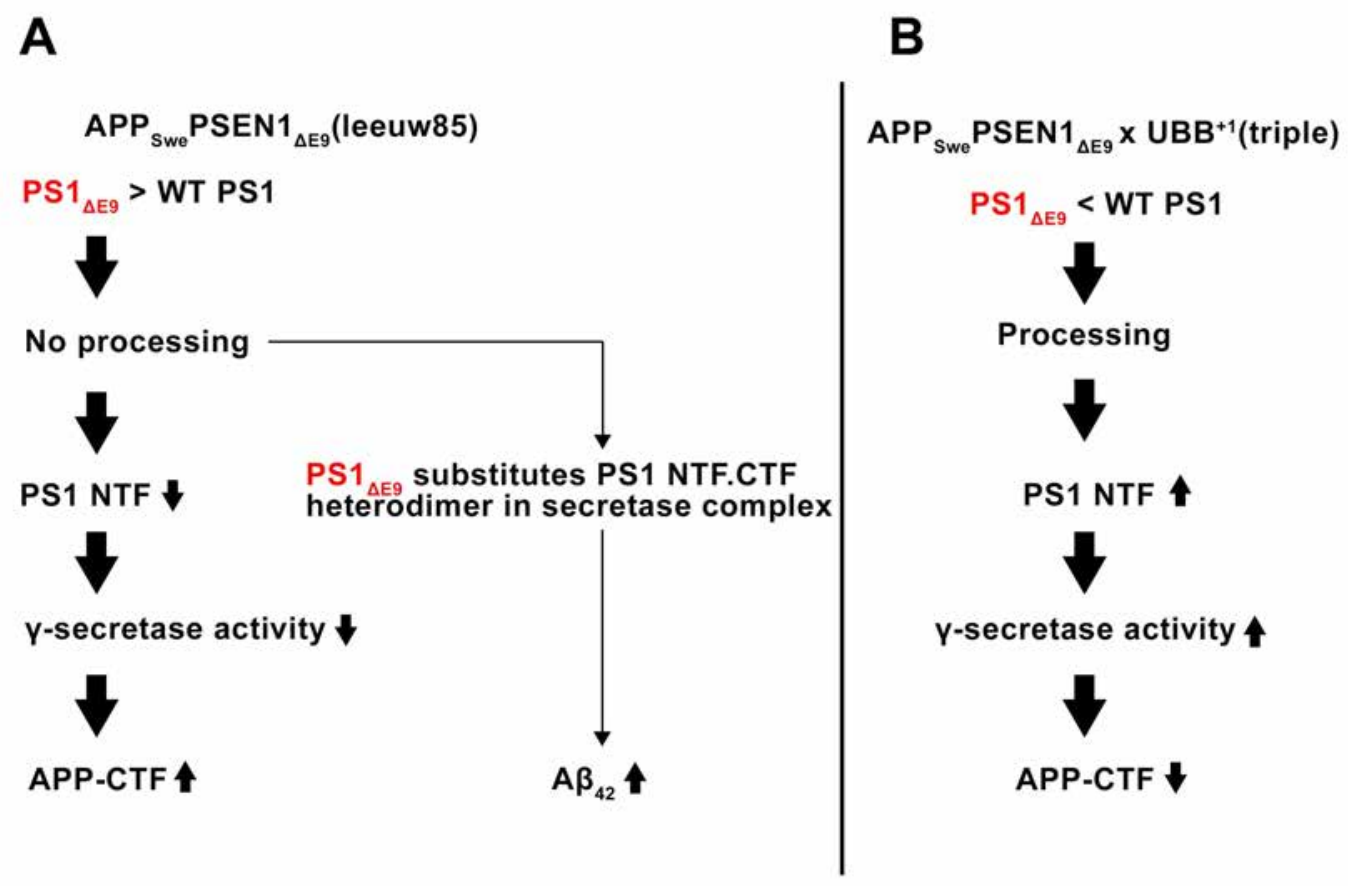

Figure 6: A) At the critical age of 6 months, APPPS1/UBB ${ }^{+1}$ mice contain more PS1 $\triangle E 9$ compared to WT PS1, leading to less PS1-NTF, decreased $\gamma$-secretase activity and less APP-CTF cleavage. Moreover, PS1 1 Eg substitutes PS1 NTF/CTF heterodimer, resulting in increased $A \beta_{42}$ proportion. $\left.B\right)$ In the APPPS1 mouse line, less mutant PS1 $\triangle E 9$ is present compared to PS1-WT. Therefore, PS1WT will be processed into NTF and CTF, resulting in enhanced $y$-secretase activity and APP-CTF cleavage compared to APPPS1.

Since the above mentioned theory depends on an impaired UPS, it is important to elucidate the relation between $A \beta$ and the UPS. Recent evidence suggest that $A \beta_{42}$ can diffuse passively from the lumen of the ER into the cytosol, where it could be degraded by the proteasome (Saido and Leissring, 2012). Consequently, impaired proteasomal activity could lead to enhanced levels of $A \beta_{42}$ in APPPS1/UBB ${ }^{+1}$ mice compared to APPPS1 mice. To the contrary, APPPS1/UBB ${ }^{+1}$ mice show a decreased $A \beta$ plaque load and soluble $A \beta_{42}$ levels (van Tijn et al., 2012). Meanwhile, $A \beta$ has been shown to decrease UPS activity in neurons in an APP mutant tg AD mouse model (Almeida et al., 2006). Therefore, high $A \beta$ plaque load and soluble $A \beta_{42}$ levels might impair PS1 degradation by the proteasome and thus enhance $y$-secretase activity levels in APPPS1/UBB ${ }^{+1}$ mice compared to APPPS1 
mice at 6 months of age. Nonetheless, the explanation of an impaired proteasome is debatable, because APPPS1/UBB ${ }^{+1}$ mice do not show reduced proteasomal activity compared to APPPS1 mice (van Tijn et al., 2012). However, it is important to note that in the above mentioned study, only chymotrypsin-like activity has been measured to indicate proteasomal activity and activity levels were measured in a whole hemisphere and not selectively in the cerebral cortex as done before where moderate proteasomal inhibition was found (Fischer et al., 2009).

Apart from possible mechanisms to explain the contrast in amyloid pathology between APPPS1 and APPPS1/UBB ${ }^{+1}$ mouse lines, it is important to discuss whether reducing A $\beta$ plaque load is a beneficial process for neuronal activity. Currently, several treatments to reduce $A \beta$ plaque formation are under investigation. $A$ potential treatment approach is immunotherapy in which antibodies directed against $A \beta$ plaques are used. However, recent clinical trials showed no cognitive improvements after immunotherapy in AD patients, probably due to the fact that amyloid pathology starts before cognitive decline can be observed (Lannfelt et al., 2014). In studies with transgenic animals, it was indeed shown that there is limited clearance of pre-existing amyloid plaques (Tucker et al., 2008). The main question that arises from these data is whether the removal of $A \beta$ plaques is relevant for curing $A D$ or just more harmful for brain function. The present results do not support the idea that a lower $A \beta$ plaque load rescues cognitive impairment in $A D$.

In the present study, no differences in synoviolin, RER1, nicastrin and N-cadherin protein levels have been identified between mouse lines at 3, 6 and 9 months of age (see General Discussion). According to the hypothesis, $\mathrm{UBB}^{+1}$ induces proteasomal inhibition and the expectation was that the increased $\mathrm{y}$-secretase activity in APPPS1/UBB ${ }^{+1}$ mice is linked to decreased levels of RER1 and increased levels of synoviolin (Tanabe et al., 2012). Moreover, it was predicted that synoviolin and RER1 influence nicastrin levels, either by inducing degradation (Maeda et al., 2009) or retrieval to ER (Spasic et al., 2007). A possible explanation for this discrepancy is that the effects of synoviolin on RER1 and nicastrin are based on cell culture experiments (Maeda et al., 2009;Tanabe et al., 2012). In the present study, hemispheres were used instead of cell lines in the Western blot experiments. These proteins are involved in several complex molecular mechanisms and different brain regions 
probably may compensate for localized significant effects. However it is still possible to find significant differences if separate brain regions were measured. For example, an immunohistochemical staining of the same transgenic mouse lines with RER75 antibody suggests that RER1 accumulates at certain locations including the hippocampus. Photomicrographs and a rationale regarding these proteins are presented in the General Discussion, Chapter 7 of the present thesis).

\subsection{Behavioral experiments: 9 months}

The behavioral data of 9-month-old male mice demonstrated that APPPS1 mice showed deficits in their spatial memory (MWM) while APPPS1/UBB ${ }^{+1}$ mice perform more poorly in nest building, were more anxious in the $Y$-maze experiment, were more active during the Y-maze, and showed deficits in working memory (Y-maze) and spatial memory (MWM). As a conclusion, the APPPS1 mice performed poorly in contextual behavior compared to WT mice. However, APPPS1/UBB ${ }^{+1}$ mice performed more poorly, apparently due to proteasomal inhibition by $\mathrm{UBB}^{+1}$.

\section{Conclusion}

The present study demonstrated that a decrease in $A \beta$ plaque load in the crossbreed caused by $\mathrm{UBB}^{+1}$ is accompanied by a rise in $\mathrm{Y}$-secretase activity and PS1 expression. According to the current scientific paradigm, the $Y$-secretase activity needs to be inhibited as PS1 is responsible for the cleavage of APP-CTF and thereby creating $A \beta$ species accumulating in $A \beta$ plaques. However, the use of $Y$-secretase inhibitors as a putative therapeutic for AD have so far been unsuccessful (De Strooper, 2014). Based on the results of the present paper, we suggest investigating exactly the opposite: the activation or at least preservation of $\mathrm{y}$-secretase activity. The idea to avoid inhibition of the $\mathrm{y}$-secretase activity, rather than inhibiting it, is in agreement with a recent study (Barnwell et al., 2014). A decreased $A \beta$ plaque load does not result in rescue of cognitive behavior, illustrating the poor predictive power of $A \beta$ load. Nevertheless, an increment of $\mathrm{Y}$-secretase activity as early as possible is still an option to improve memory function. 


\section{References}

Almeida, C.G., Takahashi, R.H., and Gouras, G.K. (2006). Beta-amyloid accumulation impairs multivesicular body sorting by inhibiting the ubiquitin-proteasome system. J Neurosci 26, 4277-4288.

Barnwell, E., Padmaraju, V., Baranello, R., Pacheco-Quinto, J., Crosson, C., Ablonczy, Z., Eckman, E., Eckman, C.B., Ramakrishnan, V., Greig, N.H., Pappolla, M.A., and Sambamurti, K. (2014). Evidence of a novel mechanism for partial gamma-secretase inhibition induced paradoxical increase in secreted amyloid beta protein. PLoS One 9, e91531.

Braun, R.J., Sommer, C., Leibiger, C., Gentier, R.J., Dumit, V.I., Paduch, K., Eisenberg, T., Habernig, L., Trausinger, G., Magnes, C., Pieber, T., Sinner, F., Dengjel, J., Van Leeuwen, F.W., Kroemer, G., and Madeo, F. (2015). Accumulation of Basic Amino Acids at Mitochondria Dictates the Cytotoxicity of Aberrant Ubiquitin. Cell Reports 9, 1557-1571.

Colmers, W.F., and El Bahh, B. (2003). Neuropeptide Y and Epilepsy. Epilepsy Curr 3, 53-58.

Couch, B.A., Kerrisk, M.E., Kaufman, A.C., Nygaard, H.B., Strittmatter, S.M., and Koleske, A.J. (2013). Delayed amyloid plaque deposition and behavioral deficits in outcrossed AbetaPP/PS1 mice. J Comp Neurol 521, 1395-1408.

De Pril, R., Fischer, D.F., Maat-Schieman, M.L., Hobo, B., De Vos, R.A., Brunt, E.R., Hol, E.M., Roos, R.A., and Van Leeuwen, F.W. (2004). Accumulation of aberrant ubiquitin induces aggregate formation and cell death in polyglutamine diseases. Human molecular genetics 13, 18031813.

De Strooper, B. (2014). Lessons from a failed gamma-secretase Alzheimer trial. Cell 159, 721-726.

De Strooper, B., Iwatsubo, T., and Wolfe, M.S. (2012). Presenilins and gamma-secretase: structure, function, and role in Alzheimer Disease. Cold Spring Harb Perspect Med 2, a006304.

Deacon, R.M., Cholerton, L.L., Talbot, K., Nair-Roberts, R.G., Sanderson, D.J., Romberg, C., Koros, E., Bornemann, K.D., and Rawlins, J.N. (2008). Age-dependent and -independent behavioral deficits in Tg2576 mice. Behav Brain Res 189, 126-138.

Dennissen, F.J., Kholod, N., and Van Leeuwen, F.W. (2012). The ubiquitin proteasome system in neurodegenerative diseases: culprit, accomplice or victim? Progress in neurobiology 96, 190-207.

Fischer, D.F., De Vos, R.A., Van Dijk, R., De Vrij, F.M., Proper, E.A., Sonnemans, M.A., Verhage, M.C., Sluijs, J.A., Hobo, B., Zouambia, M., Steur, E.N., Kamphorst, W., Hol, E.M., and Van Leeuwen, F.W. (2003). Disease-specific accumulation of mutant ubiquitin as a marker for proteasomal dysfunction in the brain. FASEB journal : official publication of the Federation of American Societies for Experimental Biology 17, 2014-2024.

Fischer, D.F., Van Dijk, R., Van Tijn, P., Hobo, B., Verhage, M.C., Van Der Schors, R.C., Li, K.W., Van Minnen, J., Hol, E.M., and Van Leeuwen, F.W. (2009). Long-term proteasome dysfunction in the mouse brain by expression of aberrant ubiquitin. Neurobiology of Aging 30, 847-863.

Fraser, P.E., Levesque, G., Yu, G., Mills, L.R., Thirlwell, J., Frantseva, M., Gandy, S.E., Seeger, M., Carlen, P.L., and St George-Hyslop, P. (1998). Presenilin 1 is actively degraded by the 26S proteasome. Neurobiol Aging 19, S19-21. 
Garcia-Alloza, M., Robbins, E.M., Zhang-Nunes, S.X., Purcell, S.M., Betensky, R.A., Raju, S., Prada, C., Greenberg, S.M., Bacskai, B.J., and Frosch, M.P. (2006). Characterization of amyloid deposition in the APPswe/PS1dE9 mouse model of Alzheimer disease. Neurobiol Dis 24, 516-524.

Glenner, G.G., and Wong, C.W. (1984). Alzheimer's disease and Down's syndrome: sharing of a unique cerebrovascular amyloid fibril protein. Biochem Biophys Res Commun 122, 11311135.

Grimm, M.O., Haupenthal, V.J., Rothhaar, T.L., Zimmer, V.C., Grosgen, S., Hundsdorfer, B., Lehmann, J., Grimm, H.S., and Hartmann, T. (2013). Effect of Different Phospholipids on alpha-Secretase Activity in the Non-Amyloidogenic Pathway of Alzheimer's Disease. Int $J$ Mol Sci 14, 5879-5898.

Haass, C., Kaether, C., Thinakaran, G., and Sisodia, S. (2012). Trafficking and proteolytic processing of APP. Cold Spring Harb Perspect Med 2, a006270.

Hardy, J., and Selkoe, D.J. (2002). The amyloid hypothesis of Alzheimer's disease: progress and problems on the road to therapeutics. Science 297, 353-356.

Holmes, C., Boche, D., Wilkinson, D., Yadegarfar, G., Hopkins, V., Bayer, A., Jones, R.W., Bullock, R., Love, S., Neal, J.W., Zotova, E., and Nicoll, J.A. (2008). Long-term effects of Abeta42 immunisation in Alzheimer's disease: follow-up of a randomised, placebo-controlled phase I trial. Lancet 372, 216-223.

Hong, L., Huang, H.C., and Jiang, Z.F. (2014). Relationship between amyloid-beta and the ubiquitinproteasome system in Alzheimer's disease. Neurol Res 36, 276-282.

Hsiao, K.K., Borchelt, D.R., Olson, K., Johannsdottir, R., Kitt, C., Yunis, W., Xu, S., Eckman, C., Younkin, S., Price, D., and Et Al. (1995). Age-related CNS disorder and early death in transgenic FVB/N mice overexpressing Alzheimer amyloid precursor proteins. Neuron 15, 1203-1218.

Igap (2015). International Genomics of Alzheimer's Disease Consortium. Convergent genetic and expression data implicate immunity in Alzheimer's disease. Alzheimer's \& Dementia 11, 658-671.

Irmler, M., Gentier, R.J., Dennissen, F.J., Schulz, H., Bolle, I., Holter, S.M., Kallnik, M., Cheng, J.J., Klingenspor, M., Rozman, J., Ehrhardt, N., Hermes, D.J., Gailus-Durner, V., Fuchs, H., Hrabe De Angelis, M., Meyer, H.E., Hopkins, D.A., Van Leeuwen, F.W., and Beckers, J. (2012). Long-term proteasomal inhibition in transgenic mice by UBB(+1) expression results in dysfunction of central respiration control reminiscent of brainstem neuropathology in Alzheimer patients. Acta neuropathologica 124, 187-197.

Jankowsky, J.L., Fadale, D.J., Anderson, J., Xu, G.M., Gonzales, V., Jenkins, N.A., Copeland, N.G., Lee, M.K., Younkin, L.H., Wagner, S.L., Younkin, S.G., and Borchelt, D.R. (2004). Mutant presenilins specifically elevate the levels of the 42 residue beta-amyloid peptide in vivo: evidence for augmentation of a 42-specific gamma secretase. Hum Mol Genet 13, 159-170.

Jankowsky, J.L., Slunt, H.H., Ratovitski, T., Jenkins, N.A., Copeland, N.G., and Borchelt, D.R. (2001). Co-expression of multiple transgenes in mouse CNS: a comparison of strategies. Biomol Eng 17, 157-165.

Krutauz, D., Reis, N., Nakasone, M.A., Siman, P., Zhang, D., Kirkpatrick, D.S., Gygi, S.P., Brik, A., Fushman, D., and Glickman, M.H. (2014). Extended ubiquitin species are protein-based DUB inhibitors. Nat Chem Biol 10, 664-670. 
Lannfelt, L., Moller, C., Basun, H., Osswald, G., Sehlin, D., Satlin, A., Logovinsky, V., and Gellerfors, P. (2014). Perspectives on future Alzheimer therapies: amyloid-beta protofibrils - a new target for immunotherapy with BAN2401 in Alzheimer's disease. Alzheimers Res Ther 6, 16.

Layfield, R., Cavey, J.R., and Lowe, J. (2003). Role of ubiquitin-mediated proteolysis in the pathogenesis of neurodegenerative disorders. Ageing Res Rev 2, 343-356.

Liu, Y., Hettinger, C.L., Zhang, D., Rezvani, K., Wang, X., and Wang, H. (2014). The proteasome function reporter GFPu accumulates in young brains of the APPswe/PS1dE9 Alzheimer's disease mouse model. Cell Mol Neurobiol 34, 315-322.

Maeda, T., Marutani, T., Zou, K., Araki, W., Tanabe, C., Yagishita, N., Yamano, Y., Amano, T., Michikawa, M., Nakajima, T., and Komano, H. (2009). An E3 ubiquitin ligase, Synoviolin, is involved in the degradation of immature nicastrin, and regulates the production of amyloid beta-protein. FEBS J 276, 5832-5840.

Manavalan, A., Mishra, M., Feng, L., Sze, S.K., Akatsu, H., and Heese, K. (2013). Brain site-specific proteome changes in aging-related dementia. Experimental \& Molecular Medicine 45, e39.

Minkeviciene, R., Rheims, S., Dobszay, M.B., Zilberter, M., Hartikainen, J., Fulop, L., Penke, B., Zilberter, Y., Harkany, T., Pitkanen, A., and Tanila, H. (2009). Amyloid beta-induced neuronal hyperexcitability triggers progressive epilepsy. J Neurosci 29, 3453-3462.

Mori, H., Kondo, J., and Ihara, Y. (1987). Ubiquitin is a component of paired helical filaments in Alzheimer's disease. Science 235, 1641-1644.

Perry, G., Mulvihill, P., Fried, V.A., Smith, H.T., Grundke-lqbal, I., and Iqbal, K. (1989). Immunochemical properties of ubiquitin conjugates in the paired helical filaments of Alzheimer disease. J Neurochem 52, 1523-1528.

Saido, T., and Leissring, M.A. (2012). Proteolytic degradation of amyloid beta-protein. Cold Spring Harb Perspect Med 2, a006379.

Schipanski, A., Oberhauser, F., Neumann, M., Lange, S., Szalay, B., Krasemann, S., Van Leeuwen, F.W., Galliciotti, G., and Glatzel, M. (2014). The lectin OS-9 delivers mutant neuroserpin to endoplasmic reticulum associated degradation in familial encephalopathy with neuroserpin inclusion bodies. Neurobiol Aging 35, 2394-2403.

Shemer, I., Holmgren, C., Min, R., Fulop, L., Zilberter, M., Sousa, K.M., Farkas, T., Hartig, W., Penke, B., Burnashev, N., Tanila, H., Zilberter, Y., and Harkany, T. (2006). Non-fibrillar beta-amyloid abates spike-timing-dependent synaptic potentiation at excitatory synapses in layer 2/3 of the neocortex by targeting postsynaptic AMPA receptors. Eur J Neurosci 23, 2035-2047.

Smith, P.K., Krohn, R.I., Hermanson, G.T., Mallia, A.K., Gartner, F.H., Provenzano, M.D., Fujimoto, E.K., Goeke, N.M., Olson, B.J., and Klenk, D.C. (1985). Measurement of protein using bicinchoninic acid. Anal Biochem 150, 76-85.

Spasic, D., and Annaert, W. (2008). Building gamma-secretase: the bits and pieces. J Cell Sci 121, 413-420.

Spasic, D., Raemaekers, T., Dillen, K., Declerck, I., Baert, V., Serneels, L., Fullekrug, J., and Annaert, W. (2007). Rer1p competes with APH-1 for binding to nicastrin and regulates gammasecretase complex assembly in the early secretory pathway. J Cell Biol 176, 629-640.

Tanabe, C., Maeda, T., Zou, K., Liu, J., Liu, S., Nakajima, T., and Komano, H. (2012). The ubiquitin ligase synoviolin up-regulates amyloid beta production by targeting a negative regulator of gamma-secretase, Rer1, for degradation. J Biol Chem 287, 44203-44211. 
Tsakiri, E.N., and Trougakos, I.P. (2015). The amazing ubiquitin-proteasome system: structural components and implication in aging. Int Rev Cell Mol Biol 314, 171-237.

Tucker, S.M., Borchelt, D.R., and Troncoso, J.C. (2008). Limited clearance of pre-existing amyloid plaques after intracerebral injection of Abeta antibodies in two mouse models of Alzheimer disease. J Neuropathol Exp Neurol 67, 30-40.

Van Gassen, G., De Jonghe, C., Pype, S., Van Criekinge, W., Julliams, A., Vanderhoeven, I., Woodrow, S., Beyaert, R., Huylebroeck, D., and Van Broeckhoven, C. (1999). Alzheimer's disease associated presenilin 1 interacts with HC5 and ZETA, subunits of the catalytic $20 \mathrm{~S}$ proteasome. Neurobiol Dis 6, 376-391.

Van Leeuwen, F.W., De Kleijn, D.P., Van Den Hurk, H.H., Neubauer, A., Sonnemans, M.A., Sluijs, J.A., Koycu, S., Ramdjielal, R.D., Salehi, A., Martens, G.J., Grosveld, F.G., Peter, J., Burbach, H., and Hol, E.M. (1998). Frameshift mutants of beta amyloid precursor protein and ubiquitin-B in Alzheimer's and Down patients. Science 279, 242-247.

Van Leeuwen, F.W., Fischer, D.F., Kamel, D., Sluijs, J.A., Sonnemans, M.A., Benne, R., Swaab, D.F., Salehi, A., and Hol, E.M. (2000). Molecular misreading: a new type of transcript mutation expressed during aging. Neurobiol Aging 21, 879-891.

Van Leeuwen, F.W., Van Tijn, P., Sonnemans, M.A., Hobo, B., Mann, D.M., Van Broeckhoven, C., Kumar-Singh, S., Cras, P., Leuba, G., Savioz, A., Maat-Schieman, M.L., Yamaguchi, H., Kros, J.M., Kamphorst, W., Hol, E.M., De Vos, R.A., and Fischer, D.F. (2006). Frameshift proteins in autosomal dominant forms of Alzheimer disease and other tauopathies. Neurology 66, S86-92.

Van Tijn, P., De Vrij, F.M., Schuurman, K.G., Dantuma, N.P., Fischer, D.F., Van Leeuwen, F.W., and Hol, E.M. (2007). Dose-dependent inhibition of proteasome activity by a mutant ubiquitin associated with neurodegenerative disease. Journal of Cell Science 120, 1615-1623.

Van Tijn, P., Dennissen, F.J., Gentier, R.J., Hobo, B., Hermes, D., Steinbusch, H.W., Van Leeuwen, F.W., and Fischer, D.F. (2012). Mutant ubiquitin decreases amyloid beta plaque formation in a transgenic mouse model of Alzheimer's disease. Neurochemistry international 61, 739748.

Vossel, K.A., Beagle, A.J., Rabinovici, G.D., Shu, H., Lee, S.E., Naasan, G., Hegde, M., Cornes, S.B., Henry, M.L., Nelson, A.B., Seeley, W.W., Geschwind, M.D., Gorno-Tempini, M.L., Shih, T., Kirsch, H.E., Garcia, P.A., Miller, B.L., and Mucke, L. (2013). Seizures and epileptiform activity in the early stages of Alzheimer disease. JAMA Neurol 70, 1158-1166.

Woodruff, G., Young, J.E., Martinez, F.J., Buen, F., Gore, A., Kinaga, J., Li, Z., Yuan, S.H., Zhang, K., and Goldstein, L.S. (2013). The presenilin-1 DeltaE9 mutation results in reduced gamma-secretase activity, but not total loss of PS1 function, in isogenic human stem cells. Cell Rep 5, 974-985. 



\section{CHAPTER 7}

\section{General discussion}




\section{The Ubiquitin Proteasome System and UBB ${ }^{+1}$}

Proteins are essential body constituents and their level is kept at equilibrium by controlling the production as well as the degradation of the proteins. The unfolded protein response (UPR) (1), proteasomal degradation (2) and autophagy (3) are the major major cellular pathways responsible for the degradation and recycling of excessive or damaged proteins to maintain cellular homeostasis. 1) The endoplasmatic reticulum (ER) of mammals is comprised of three ER transmembrane sensors to check the ER protein folding. These sensors activate the UPR if protein degradation is necessary or triggers programmed cell death if the ER homeostasis is fatally damaged. Strict regulation of the UPR pathway is necessary to prevent inappropriate activation in response to innocuous stimuli (Karam et al., 2015). Recently, it was described that the nonsense-mediated RNA decay (NMD) is involved in this regulation by increasing the UPR activation threshold and by promoting its timely decrease (Karam et al., 2015). 2) The Ubiquitin Proteasome System (UPS) is another protein degradation system which is ATP-dependent. The discovery of UPS occurred in two steps. a) ATP-dependent proteolytic factor 1 (APF-1), later defined as ubiquitin (UBB), was shown to modify covalently proteins which led to degradation of the targeted protein. b) The $26 \mathrm{~S}$ proteasome, which is the active proteolytic complex responsible for the degradation of the targeted protein was discovered (Ciechanover, 2014). 3) An alternative pathway is autophagy which is a lysosome-dependent degradation pathway. The autophagy pathway acts as a compensatory pathway when the proteasome is impaired (Kageyama et al., 2014).

Ubiquitination of proteins play a role in a wide variety of functions: proteasomal dysfunction as mentioned above, but also in regulation of signal transduction pathways, endocytosis, neuronal functioning and embryonic neural development. For example, a decreased number of neural stem cells (NSCs) and an increased number of glial cells was recently shown in cultured cells isolated from $\mathrm{UBB}^{-1-}$ mouse embryonic brains. This was due to reduced proliferation of NSCs and to the dysregulated timing of gliogenesis and therefore NSCs in these mice acquired higher numbers of gliogenic cells than neurogenic cells. These data point to the pivotal role of ubiquitin in embryonic neural development because its cellular level is an important determinant in controlling the fate and timing of NSC 
differentiation and has influence on the self-renewal of NSCs (Ryu et al., 2014). Over the years, many missing pieces regarding the structure and function of the UPS and ubiquitin were discovered and this is still an ongoing process. Recently, two independent studies pointed at the prominent role of the UPS in (Alzheimer's disease) (AD). Genome Wide Association Studies (GWAS) and pathway analysis showed that protein ubiquitination represents a prime target for AD therapeutics (IGAP, 2015). In addition, an Ingenuity Pathway Analysis enhanced the position of a dysfunctional UPS as a causative factor in AD by showing 31 proteins which were significantly altered and they had a strong interaction with Ubiquitin C (UBC) signaling (Manavalan et al., 2013). The present thesis investigates the aberrant protein ubiquitin $\mathrm{B}^{+1}\left(\mathrm{UBB}^{+1}\right)$ which can cause a dysfunctional UPS and therefore accumulation of proteins. The crystal structure of this misframed protein is shown in (Figure 1).

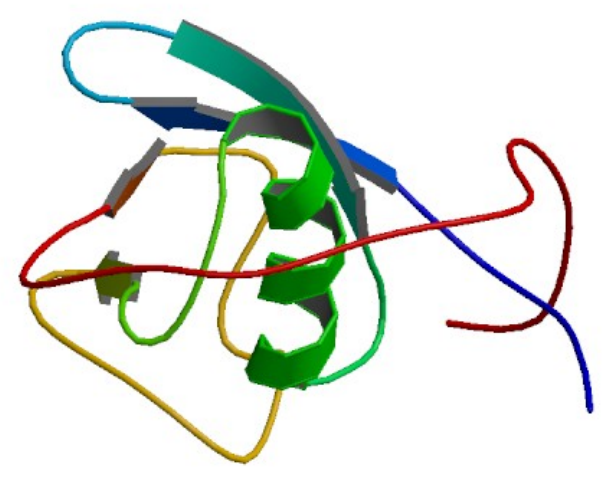

Figure 1: Crystal structure of the $U B B^{+1}$ protein. $U B B^{+1} m R N A$ is formed during transcription from the UBB locus by a process called "molecular misreading". Dinucleotide deletions $(\triangle G U)$ in the UBB transcript occur at the C-terminal part next to a GAGAG motif which result in a +1 reading frame shift and finally in an altered protein sequence. The resulting $U B B^{+1}$ protein has a 20 amino acid extension (red color) and lacks the C-terminal glycine which is required for conjugation to target proteins for degradation. This figure is derived from the Protein Data Bank (DOI: 10.2210/pdb2kx0/pdb). 
In general, it is assumed that DNA sequences are copied identically into RNA. However, there are exceptions to this one-to-one relationship between RNA and its corresponding DNA sequences. Recently, 12 types of RNA-DNA sequence differences (RDDs) were found in nascent RNA and these RDD's occur very soon after transcription (Li et al., 2011). These data described sequence substitution as an early step in co-transcriptional RNA processing (Li et al., 2011;Wang et al., 2014). However also in the translation of RNA into proteins there is not necessarily a 1:1 relationship between the expression levels. For example, the supplementary data of Chapter 3 showed the results of quantitative qPCR analysis in the hippocampal formation in brains of $\mathrm{UBB}^{+1}$ transgenic (tg) and wild type (WT) mice. This experiment was performed to study the expression of a panel of genes involved in AD, UPS and pre- or postsynaptic functioning. Of the 35 genes tested, 7 were significantly upregulated in the $\mathrm{UBB}^{+1} \operatorname{tg}$ mice versus controls. They consist of 5 proteins involved in synaptic functioning (GRIN2A, CamK2A, SNAP25, GABRB2 and GLUR2), as well as 14-3-3 $\zeta$ and amyloid precursor protein (APP) (Figure 2A). In a subsequent step, the protein levels of those genes were analyzed by Western blotting (Figure 2B). In contrast to the transcript data, most of the synaptic proteins did not show a significant difference at the protein level or were even regulated in the opposite direction (e.g., GABRB2) (Wang, 2008).

A

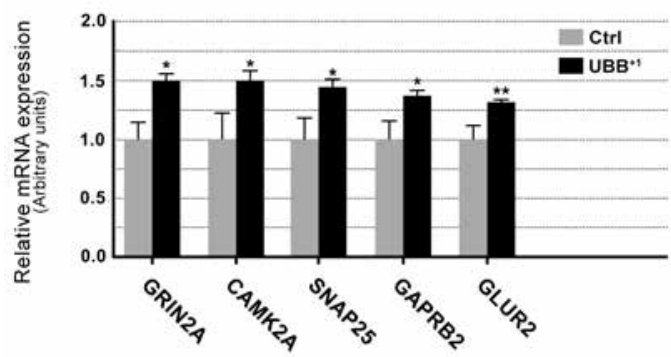

B

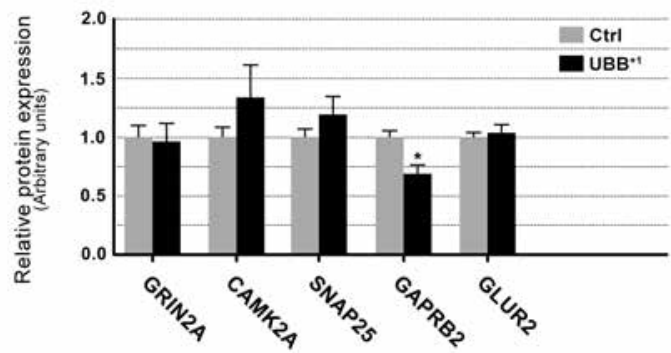

Figure 2: Differentially regulated genes in the hippocampus of $U B B^{+1} \operatorname{tg}$ mice versus age-matched controls (9 months) at mRNA (A) and protein (B) levels. A) Five genes involved in synaptic functioning were upregulated in the hippocampus of UBB ${ }^{+1}$ mice compared with WT mice: GRIN2A (151\%), CAMK2A (151\%), SNAP25 (143\%), GABRB2 (134\%) and GLUR2 (129\%). B) An increment in gene 
expression does not automatically result in an increase at protein level. GRIN2A, CAMK2A, SNAP25 and GLUR2 did not show any difference between the $U B B^{+1}$ and WT mice. For GABRB2 even an opposite (decreased) protein expression level was found compared with the mRNA levels. Error bars represent SEM. ${ }^{*} p<0.05,{ }^{* *} p<0.01$.

One of the main features of the present thesis is the demonstration by topographic mapping that $\mathrm{UBB}^{+1}$ not only has a role in the cognitive problems of $A D$ but also in comorbidities associated with AD (Chapters 2 and 3). The nucleus of the solitary tract (NTS) (respiration), parabrachial nucleus (PBN) (respiration), raphe nuclei (depression), inferior colliculus (IC) (audition), olfactory bulb (OB) (olfaction), nucleus basalis of Meynert (NBM) and basal ganglia (brain-reward system) were brain regions in the $3413 \mathrm{tg}$ line which showed clear $\mathrm{UBB}^{+1}$ expression. It is known from the literature that each of the functions of these regions is affected in $A D$. This suggests that $U B B^{+1}$ has a role in the comorbidities linked to $A D$ and perhaps also in other neurodegenerative diseases demonstrating comorbidities and $\mathrm{UBB}^{+1}$ spreading in the brain. In addition, Chapter 4 characterized the harmful consequences of $\mathrm{UBB}^{+1}$ presence within the cell. A dysfunctional UPS upon UBB ${ }^{+1}$ accumulation causes mitochondrial damage. The unexpected enhancement of basic amino acid synthesis is described as the decisive toxic event which can be relieved by increasing mitochondrial proteostasis. We demonstrated the presence of VCP/Cdc48-associated mitochondrial stress-responsive (VMS1), the mitochondrion-specific component of the UPS, together with $\mathrm{UBB}^{+1}$ in the hippocampus of $\mathrm{AD}$ patients. The Cdc48/Vms1-complex is able to reverse the toxic $U_{B B}^{+1}$-induced enhancement of basic amino acids which indicated that this pathway can be a novel target for preventing neuronal dysfunction in AD (Tan et al., 2007).

These data emphasize the importance of $\mathrm{UBB}^{+1}$ in basic mechanisms such as respiration (Yaffe et al., 2011) and homeostasis. In the pyramid of Maslow's hierarchy (Maslow, 1943) (Figure 3), it is shown that a human being firstly has to meet the needs at basal level of the triangle before moving up to the second step in the pyramid. 


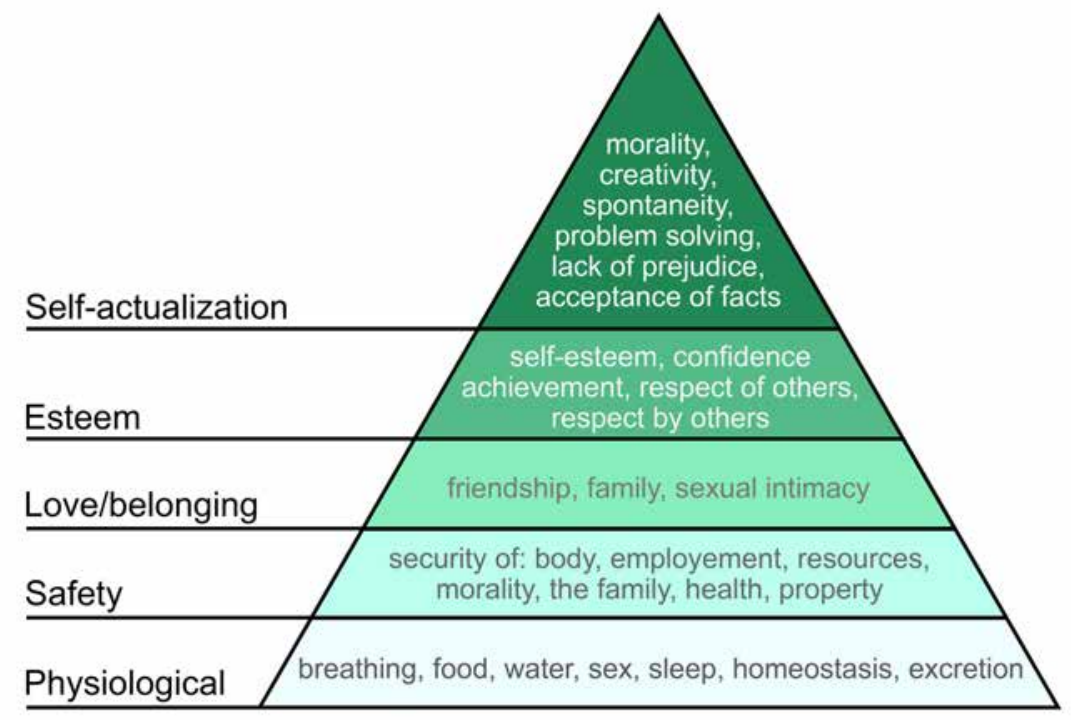

Figure 3: Maslow's hierarchy of needs. It is a psychological theory that includes 5 stages (Yaffe et al., 2011): physiological, safety, love/belonging, esteem and self-actualization to describe the pattern that human motivations generally move through. This theory is portrayed in the shape of a pyramid, with the most fundamental needs at the bottom and the self-actualization need at the top. This most fundamental needs must be met before an individual will desire the secondary level of needs (Maslow, 1943).

Another aim of the present thesis was to identify a relationship between a dysfunctional UPS environment and the widely accepted "Amyloid $\beta(A \beta)$ hypothesis". In order to investigate interactions of these two theories, a model combining them was created by means of our triple APPPS1/UBB ${ }^{+1}$ tg mice. As mentioned earlier (Chapter 5), to our surprise a decreased $A \beta$ plaque load was found in the triple $\operatorname{tg}$ mice compared with the double APPPS1 tg mice. In Chapter 6, we examined the molecular mechanisms behind this decrease in $A \beta$ plaque load and we showed a significantly decreased $Y$-secretase activity in APPPS1 at 6 months of age while this was restored to WT-levels in the APPPS1/UBB ${ }^{+1}$ mice. These differences in $\mathrm{y}^{-}$-secretase activity could be ascribed to at least changes in presenilin 1 (PS1) expression levels. Nevertheless, APPPS1/UBB ${ }^{+1}$ mice showed decreased plaque load at 6 months. Consequently, a better behavioral outcome 
was one of our expectations. However, these triple tg mice performed even worse in contextual behavior test compared to the APPPS1 mice. These data suggest that a decrease in $A \beta$ plaque load does not necessarily mean an improvement in behavioral readouts. Possibly, $A \beta$ plaque load is less relevant for curing $A D$ than previously assumed and perhaps not $A \beta$ in plaques but another form of $A \beta$ (oligomers) is the detrimental factor which has already been hinted at some other studies (Chen, 2015;Viola and Klein, 2015).

\section{$A \beta$ and Ubiquitin Proteasome System}

As mentioned in the present thesis, according to the "Amyloid cascade hypothesis" accumulation of $A \beta$ species is the earliest change and a causative factor in $A D$. Extracellular $A \beta$ plaques were seen as the damaging component in $A D$. However, recent studies suggest that intracellular $A \beta$ burden the neuronal function and extracellular plaques are a dustbin for the cells, being a way to get rid of the $A \beta$ species. $A \beta$ is degraded by the ATP-dependent ubiquitination process and the association between A $\beta$ and UPS needs to be elucidated. UBB-wt itself is present in enlarged neurites associated with neuritic plaques (NP) (Perry et al., 1987). $A \beta_{42}$ is able to diffuse passively through the ER lumen into the cytosol where it will be degraded by the proteasome and insulin degrading enzyme (IDE) (Hong et al., 2014). This was confirmed in an experiment showing that after inhibition of the $26 S$ proteasome with lactacystin a remarkable decrease in $A \beta_{42}$ degradation was detected. These data suggest that $A \beta$ species in astrocytes and neurons are a substrate for the UPS and aberrations in this system could cause increased $A \beta$ accumulation (Lopez Salon et al., 2003). A $\beta$ species itself also have their effects on the function of the $26 S$ proteasome (Zheng et al., 2014). The proteasomal activity was significantly decreased in selected brain regions of $A D$ patients probably due to brain-region specific alterations (Keller et al., 2000). $A \beta_{25-35}$ and $A \beta_{42}$ causes in neurons a significant increase in UBBconjugates and in the expression of E1 enzymes (Lopez Salon et al., 2003). It was demonstrated in vitro that $A \beta$ binds to the $20 S$ proteasome and impedes the $\beta_{5}$ chymotrypsin-like activity (Gregori et al., 1995). Earlier studies showed that $A \beta$ acts as a simple inhibitor of proteasome activity. However, more recent work suggested that the proteasomal dysfunction caused by $A \beta$ may arise from the competition of natural proteasome substrates with the expanding numbers of toxic oligomeric $A \beta$ peptides within 
the neuronal cells of AD patients (Zhao and Yang, 2010). In Tg2576 tg mice, A $\beta$ accumulation is present in the late endosome/multivesicular body where it causes impairment of the multivesicular sorting pathway via the UPS which further impairs the breakdown of epidermal growth factor receptor (EGFR) (Zheng et al., 2014). Another study showed that $A \beta$ oligomers rather than monomers inhibit the proteasome in vitro (Tseng et al., 2008). As mentioned, the misframed protein $U_{B B}^{+1}$ forms the main topic of the present thesis. It was shown earlier that $\mathrm{UBB}^{+1}$ works dose-dependently and at high doses it will inhibit the proteasome (van Tijn et al., 2007). Chapter 5 of the present thesis showed a decrease in $A \beta_{42}$ levels and $A \beta$ plaque load in a triple tg mouse model APPPS1/UBB ${ }^{+1}$ at 6 months of age.

Another factor which has impact on both $A \beta$ formation and the proteasome is $E 2-25 \mathrm{~K}$. This enzyme is an atypical E2-enzyme because it can elongate the UBB chain without support of E3-ligases. It mediates in $A \beta$ toxicity and the levels of this enzyme are upregulated in AD. Parkin, ubiquitin carboxy-terminal hydrolase isozyme L1 (UCHL-1) and HRD1/synoviolin, all E3 ligases are also associated with $A \beta$. Parkin is involved in juvenile Parkinson's disease. It has a role in the ubiquitination and clearance of $A \beta$ and localizes with intraneuronal $A \beta_{42}$ in $A D$ brains (Lonskaya et al., 2013). UCHL-1 has been claimed to degrade BACE1 which will affect the APP processing and A $\beta$ accumulation (Hong et al., 2014). UCHL-1 also functions as a deubiquitinating enzyme (DUB) that cleaves ubiquitin at its C-terminus.

Recently it was shown that UCHL-1 is responsible for an increase in free ubiquitin levels and it is involved in the lysosomal degradation of APP by promoting its ubiquitination. It was demonstrated that an overexpression of UCHL-1 by intracranial injection causes a reduction in the $A \beta$ production, an inhibition of NP formation and an improvement in memory deficits in an AD tg mouse model (Zhang et al., 2014). In AD, HRD1/synoviolin is insolubilized which causes its dysfunction resulting in increased $A \beta$ levels (Kaneko et al., 2012). As a conclusion, HRD1/synoviolin levels are negatively correlated with the $A \beta$ production levels.

In Chapter 6 of the present thesis the expression of a protein dubbed "retention in endoplasmic reticulum sorting receptor 1" (RER1) and HRD1/synoviolin were measured by 
Western blot in WT, UBB ${ }^{+1}$, APPPS1 and APPPS1/UBB ${ }^{+1}$ mice at 3, 6 and 9 months of age. RER1 is known as the negative regulator of the stepwise assembly of the $\mathrm{Y}$-secretase complex during the ER-to-Golgi transport and causes lower levels of immature APP. This mechanism tightly regulates the quantitative levels of $Y$-secretase complex and activity in post-Golgi compartments (Park et al., 2012;Jurisch-Yaksi and Annaert, 2013). HRD1/synoviolin is associated with RER1 as it is involved in the degradation of immature nicastrin and RER1 (Maeda et al., 2009;Tanabe et al., 2012). Based on these data we hypothesize an association between HRD1/synoviolin, RER1, y-secretase and A $\beta$ plaque load (Figure 4). In the APPPS1/UBB ${ }^{+1}$ mice, we detected a significantly decreased level of $A \beta$ plaque load at 6 months of age while the $\gamma$-secretase activity was increased. Based up on our data combined with the literature, we hypothesized that RER1 should be decreased and HRD1/synoviolin increased in the triple tg mice (APPPS1/UBB ${ }^{+1}$ ). In the double $\operatorname{tg}$ line 85 we expected the opposite effects. However, no significant differences were shown in the Western blot analyses and therefore we could not confirm our hypothesis. One possible explanation is that earlier published data on RER1 and synoviolin were based on cell culture experiments (Maeda et al., 2009;Tanabe et al., 2012) while we worked with half brain hemispheres. These proteins have various roles in different complex mechanisms and effects are location-dependent. Measurements of a brain hemisphere probably compensate for local significant effects. Focusing on separate brain regions would possibly detect significant effects. Indeed, RER1 accumulation was shown for example in some regions of the hippocampus by using the RER75 antibody in an immunohistochemical experiment (Figure 5).

These data taken together suggest that a clear association exists between $A \beta$ processing and the UPS. However, whether a dysfunctional UPS causes $A \beta$ accumulation or the other way around remains elusive. 


\section{$\mathrm{APP}_{\mathrm{Swe}}, \mathrm{PS}_{\triangle \mathrm{SE9}}($ line 85)}

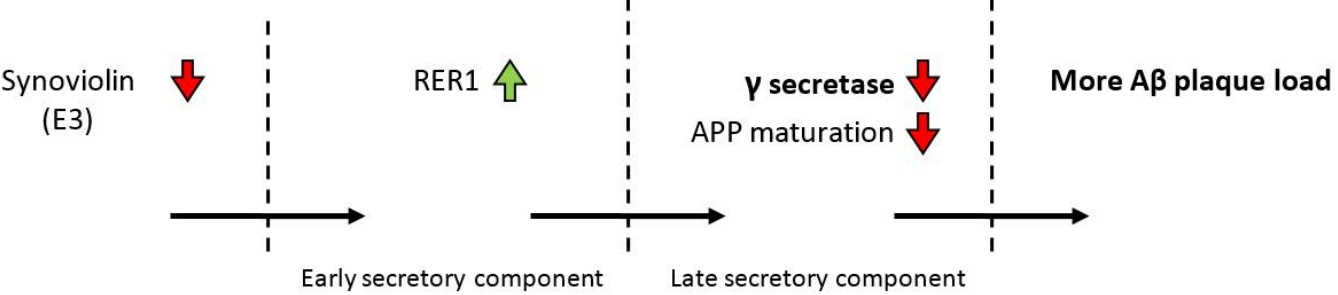

Triple $\left(\mathrm{APP}_{\mathrm{Swe}}, \mathrm{PS}_{\triangle E 9} \times \mathrm{UBB}^{+1}\right.$, line 3413)
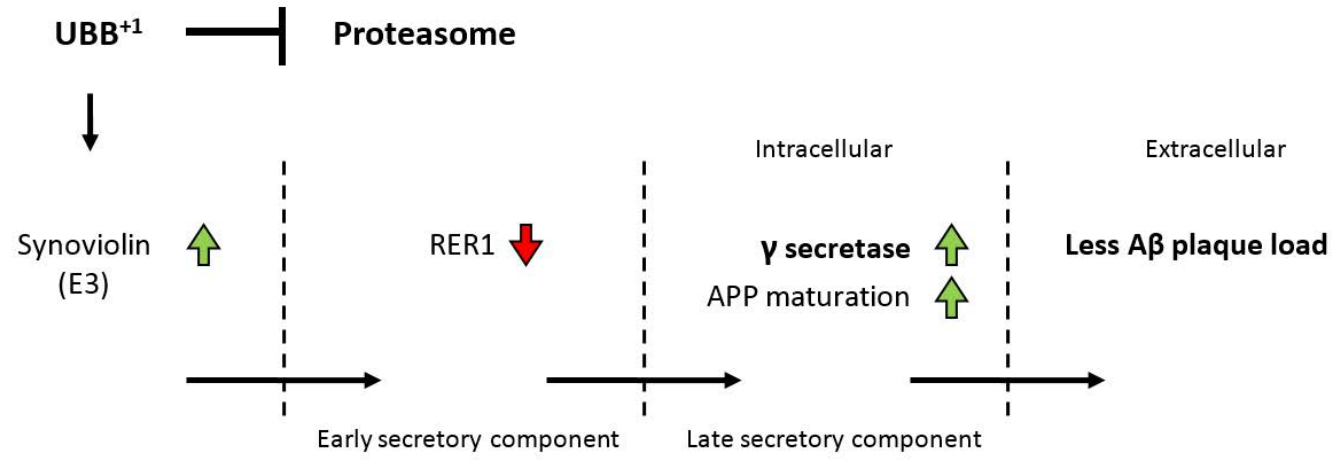

Figure 4: Schematic overview about our hypothesis regarding the association between synoviolin, RER1, $\gamma$-secretase activity and $A \beta$ plaque load. For a clear understanding it is advisable to start reading from the right side of the diagram.

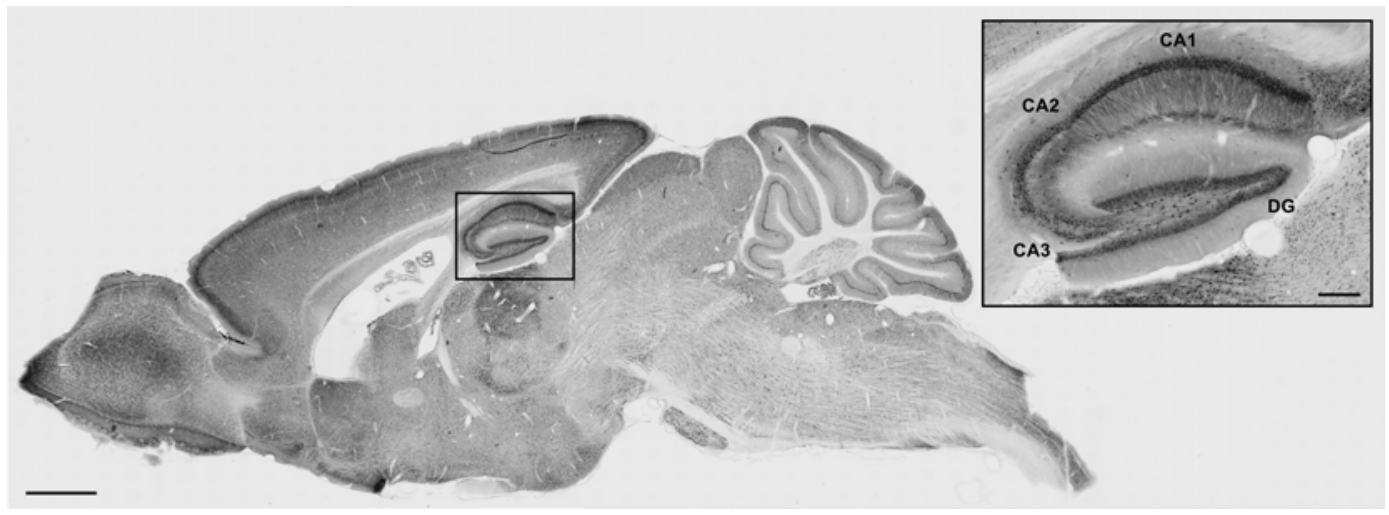

Figure 5: Expression of RER1 in a sagittal brain section $(50 \mu m)$ an UBB ${ }^{+1}$ mouse. Overall RER1 immunoreactivity Insert shows the expression of RER1 in the hippocampal formation. RER1 
immunohistochemistry was performed with antibody \#75, 1:2500 which was generously provided by Prof. T.E. Golde (Centre for Translational Research in Neurodegenerative Disease, University of Florida, Gainesville, USA). Scale bar $=1 \mathrm{~mm}$, scale bar insert $=200 \mu \mathrm{m}$.

In Chapter 2 the topographic mapping of the $\mathrm{UBB}^{+1}$ expression in the brain of $\mathrm{tg}$ mice is described and Chapter 3 shows an in-depth analysis of the $\mathrm{UBB}^{+1}$ expression in the PBN and the NTS of $\operatorname{tg}$ mice involved in respiration. These mouse data were validated in the same human $A D$ brainstem regions showing the distribution $U_{B B}^{+1}$ and misfolded tau (pretangles; MC1 antibody). In addition to these data supplementary immunohistochemical experiments were performed in the brainstem of the same AD patients using the CP13 antibody recognizing phosphorylated tau serine 202 (post-tangles) and 6F3D (M0872, raised against residue 8-17 of $A \beta$ ) recognizing amyloid $\beta$ (van Leeuwen et al., 2006). The specificity of many markers developed for AD, including the MC1 and CP13 antibodies were recently summarized (Petry et al., 2014).

\section{A $\beta$ plaque load}

As mentioned above, Chapter 3 (Irmler et al., 2012), additional immunohistochemical experiments were performed using the $6 F 3 D$ antibody to demonstrate the presence of $A \beta$ plaques in human brainstem areas. In brainstem sections from Braak stage 0 (dubbed Braak 0) patients no pathology was present apart from a single $A \beta$ plaque in the locus coeruleus (LC) while in Braak 3 patients the LC showed minor $(+)$ to considerable $(++)$ immunoreactivity. Plaque load was present in the dorsal part of the lateral parabrachial nucleus (PBNdl) and minor (+) plaque load in the caudal (PBNcau), external part (PBNel) and NTS. Also in sections of the rostral pons from a Braak 5 patient, considerable $(++)$ plaque load was present in the LC and NTS while minor (+) immunoreactivity was seen in the lateral (PBNI) and medial (PBNm) parabrachial nucleus. In the three Braak 6 patiens, the LC of the rostral pons, sagulum (SAG), PBNm, PBNcaul and PBNel showed considerable $(++)$ immunoreactivity for $A \beta$ plaques whereas PBNdl showed intense immunoreactivity for $A \beta$ plaque load (+++) (Figure 6). In contrast to the rostral pons, a slightly higher plaque load was present in the LC of the caudal pons. The PBNm is affected by a considerable (++) $A \beta$ plaque load which decreased in the external part of the medial parabrachial nucleus (PBNem). Minor $(+)$ to considerable $(++)$ immunoreactivity was 
observed in the PBNel. The Braak 6 patients were affected by $A \beta$ plaques increasing from minor immunoreactivity $(+)$ in the caudal Sol (medial (SolM) and intermediate (SolIM) part of NTS) to considerable (++) immunoreactivity in rostral sections of the caudal medullary Sol (SolM and SollM) (Figure 7).

In summary, the PBN can be affected by $A \beta$ plaques and the plaque load expanded with increasing Braak stage. The PBNI, especially the PBNdl, was generally more affected than the PBNm and also the LC was affected by $A \beta$ plaque load. The NTS shows $A \beta$ plaque load mostly (++) near the obex, but shows less $A \beta$ burden compared with other medullary regions (e.g. intermediate reticular nucleus (IRt), lateral reticular nucleus (LRt) and subtrigeminal part of the lateral reticular nucleus (LRtS5)).

\section{Neurofibrillary tangles}

To obtain a comprehensive view of the presence of tangle-like structures in the human brainstem we added another antibody called CP13 (recognizing post-tangles) to our data in Chapter 3 (Irmler et al., 2012) where only the MC1 antibody (pretangles) was tested initially. In both Braak 0 patients only light neurofibrillary tangle (NFT) density was seen in the LC area and at the border of the $4^{\text {th }}$ ventricle in the ependymal layer. Minor $\left(^{+}\right)$ immunoreactivity against NFTs was present in the NTS of the caudal and rostral medulla of Braak 0 patients. In a Braak 3 patient, the LC was affected by considerable (++) NFT density accompanied by a few NPs. The PBNI exhibited minor (+) tau-immunoreactivity, whereas the PBN, was even less affected by NFTs. Minor (+) to considerable (++) density of NFTs was observed in the LC of a Braak 5 patient while the PBNI and PBNm showed minor NFT (+) pathology. The sections of these Braak 0, 3 and 5 patients were related to the rostral pons. In contrast to that, the sections analyzed from the Braak 6 patients were derived from the rostral and the caudal pons. Regarding the rostral pons, the PBNm was remarkably affected by NFTs, whereas the PBNI showed less immunoreactivity. However, the PBNdl demonstrated considerable $(++)$ to intense $(+++)$ tau immunoreactivity. Moreover, NPs were seen around the midline. In the caudal pons, considerable (++) tau expression was seen in the PBNel while only minor (+) NFT density was present in the PBNem and minor (+) to considerable (++) in the PBNm (Figure 6). An intense (+++) NFT 
pathology was present in the caudal part of the pons of the Braak 6 patients. NFT pathology in the rostral medulla of Braak 3 samples was as sparsely as in Braak 0 patients while the SolIM was minor (+) affected. A considerable (++) amount of NFTs was present in the SolIM of Braak 6 patients throughout the entire medulla, decreasing slightly from caudal ++ to rostral $+(+)$ (Figure 7$)$.

In summary NFT pathology is increased and spread with increasing Braak stages and that the PBN is again bilaterally affected. Furthermore, the PBNI was more affected than the PBNm, and NFTs were co-localized with NTs. The density of NTs seemed to be related to the density of NFTs in the pons. The Sol of Braak 6 patients are affected moderately through the entire medulla, decreasing from caudal to rostral. NPs were very rarely seen in any sections of the medulla and NFTs were often accompanied by neuropil threads (NTs) according to their severity. As shown in Chapter 2, UBB ${ }^{+1}$ was clearly present in pontine regions as the LC and the subnuclei of the PBN and in the medullary regions as the NTS and the area postrema (AP). Strongly positive $\mathrm{UBB}^{+1}$ immunoreactive neurons were especially present in the PBNel and PBNdl, both receiving visceral inputs form the caudal NTS. These data showed again the co-incidence of $\mathrm{UBB}^{+1}$ with tangle-like structures. 


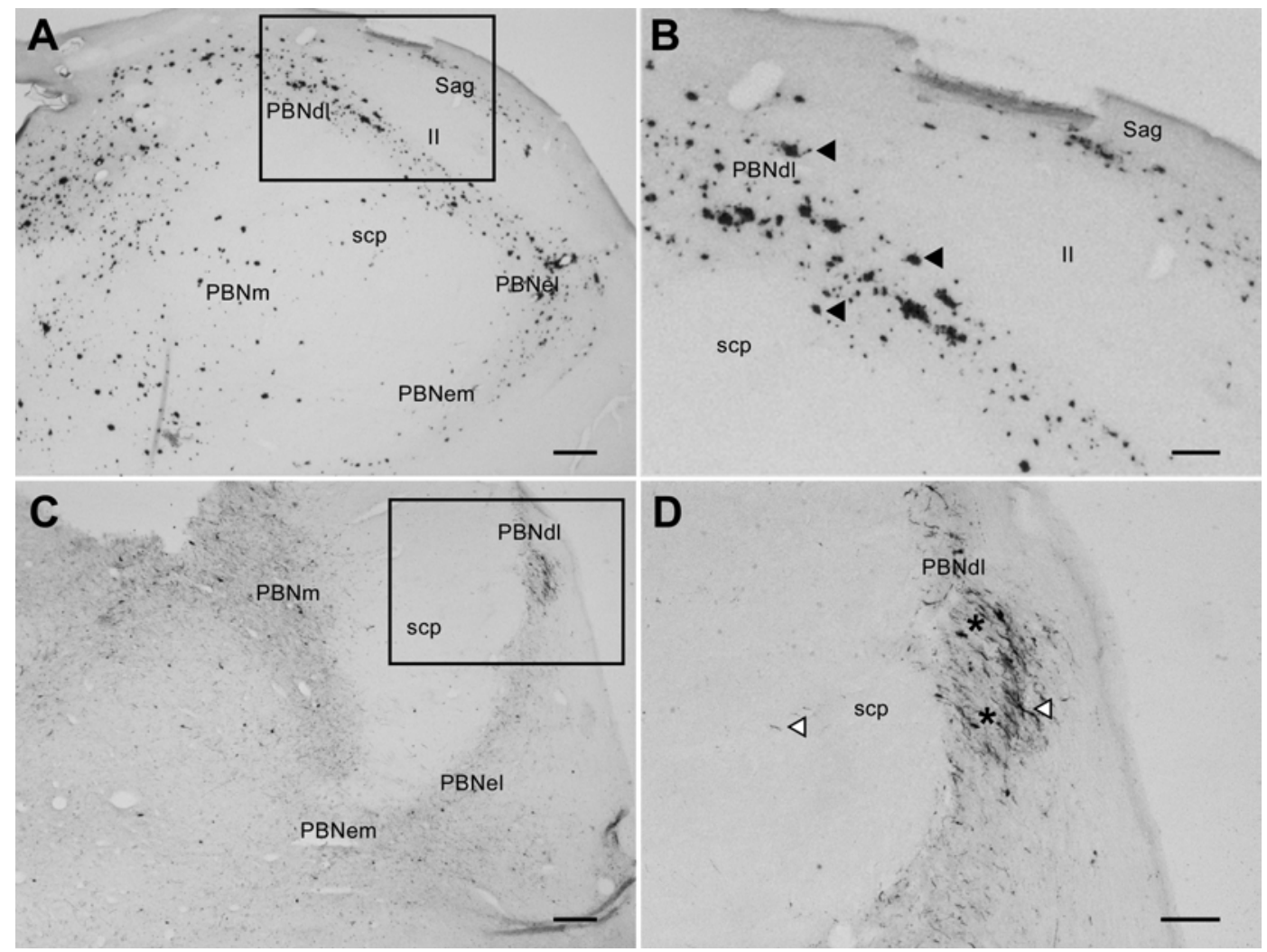

Figure 6: Immunohistochemical expression of $A \beta$ plaques (6F3D) (A-B) and tangles (CP13) (C-D) in the human PBN of an $A D$ patient (Braak stage 6). (A-B) shows $A \beta$ plaque load (++) in regions of the pontine, indicated by black arrowheads. B) presents the higher magnification picture of the boxed area in (A). (C-D) shows hyperphosphorylated tau in PBN (details, see text). Asterisks indicate the neurofibrillary tangles (NFTs) and the white arrowheads indicate neuropil threads (NTs). D) presents the higher magnification picture of the boxed area in (C). Scale bars: $A, C=500 \mu \mathrm{m} ; B, D=200 \mu \mathrm{m}$ 


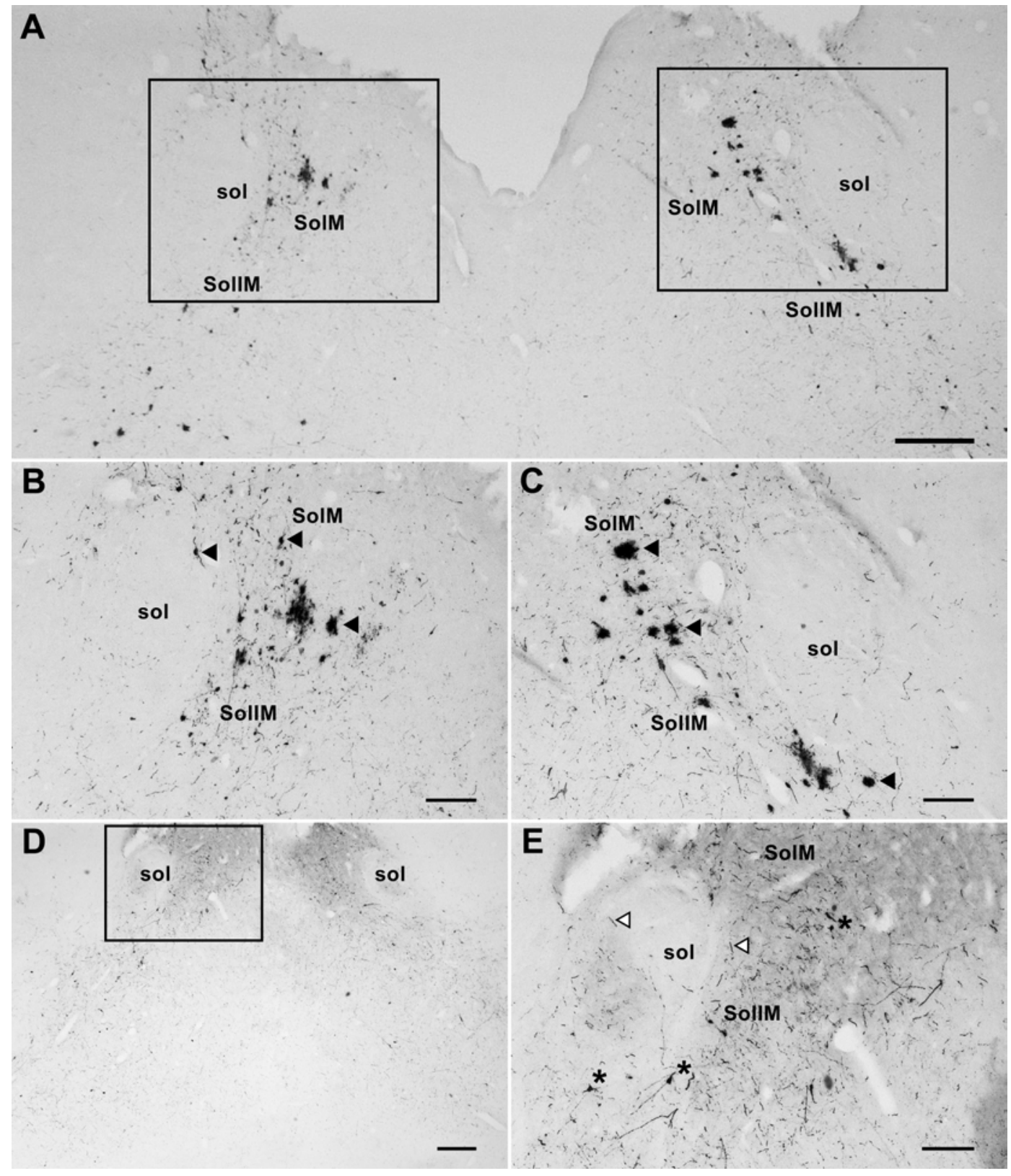

Figure 7: Immunohistochemical expression of $A \beta$ plaques (6F3D) and tangles (CP13) in the human NTS of an $A D$ patient (Braak stage 6). (A-C) shows $A \beta$ plaque load (++) in regions of the pontine $P B N$ and medullary NTS, indicated by black arrowheads. $(B)$ and $(C)$ present the higher magnification picture of the boxed areas in (A). (D-E) shows hyperphosphorylated tau in NTS (details, see text). 
Asterisks indicate the neurofibrillary tangles (NFTs) and the white arrowheads indicate neuropil threads (NTs). E) presents the higher magnification picture of the boxed area in (D). Scale bars: $A$, $D=500 \mu m ; B, C, E=200 \mu m$

\section{Epilepsy in Alzheimer's disease}

In the present thesis, the APPPS1 (line 85, APPswe PS1 ${ }_{\triangle \mathrm{E} 9}$ ) mice were used as well as the crossbreed of lines \#85 and 3413 (APPPS1/UBB ${ }^{+1}$ ). Many of the seizures recorded in these tg mice were derived from behavioral observations as well as electrographic seizures (Minkeviciene et al., 2009). It was described that in line 85 at least one electrographic seizure was detected in 25\% of the 3-month-old mice which increases to $55 \%$ at 4.5 months of age (Minkeviciene et al., 2009). In Chapter 6 of the present thesis behavioral experiments were performed on double and triple tg mice of 3, 6 and 9 months of age. Sudden deaths in these mice can occur at any age but the rate was the highest around the age of 3-4 months together with the first plaque-appearance in cortical and hippocampal areas (Garcia-Alloza et al., 2006). Consequently, aging the APPPS1 and APPPS1/UBB ${ }^{+1}$ mice until 9 months was impossible because they died earlier or they had to be sacrificed because of the detection of a seizure (protocol requirement of local animal ethical committee). Therefore, cryopreserved morula of APPPS1 line 85 were revitalized and crossed again with $\mathrm{UBB}^{+1} \mathrm{tg}$ mice. After revitalization, the double and triple $\operatorname{tg}$ mice were aged until 9 months of age without suffering of silent epileptic insults. This appears to have been due to the fact that the revitalized line received normal $(\mathrm{pH}=8)$ tap water while the mice of 3 and 6 months of age were maintained on acidified drinking $(\mathrm{pH}=2.5)$ and custom at Maastricht University, central animal facility.

Transgenic or knock-in mice are created carrying one or several mutations described in early-onset $A D$ to study $A D$ pathology which develops in humans over decades. Spontaneous seizures or sharp wave discharges (SWD) are present in a whole range of APP-overexpressing tg mice (e.g., Tg2576 (APPswe mutation with Prp promoter); APP23

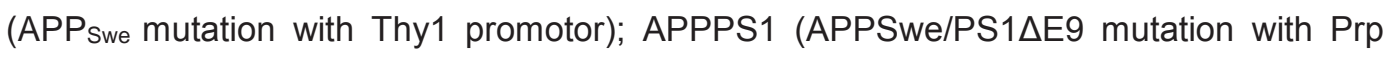
promoter) (Mucke and Selkoe, 2012;Born, 2015). Recently it was demonstrated that overexpression of 14-3-3 zeta $(\zeta)$ in tg mice resulted in a selective downregulation of UPR pathway and protects against ER stress- and seizure-induced neuronal death in the mouse 
hippocampus (Brennan et al., 2013). These data suggest a potential therapeutic approach for prolonged seizures by overexpression of 14-3-3 $\zeta$. 14-3-3 proteins are ubiquitous molecular heat-inducible chaperons, excessively expressed in the brain, and are involved in functions as cell proliferation, differentiation, metabolism and apoptosis (Brennan et al., 2013). However, aberrant expression levels of 14-3-3 proteins occur in different neurodegenerative diseases. In AD 14-3-3 $\zeta$ is found in NFTs, as well as in NTs and it was suggested that these proteins can function as sweepers of misfolded proteins (Kaneko and Hachiya, 2006). It is known that $\mathrm{UBB}^{+1}$ can induce heat shock proteins like of 14-3-3 $\zeta$; however, the molecular mechanisms behind this induction remains to be determined (Irmler et al., 2012). 14-3-3 3 is a protein which is involved in both AD pathology as in epilepsy.
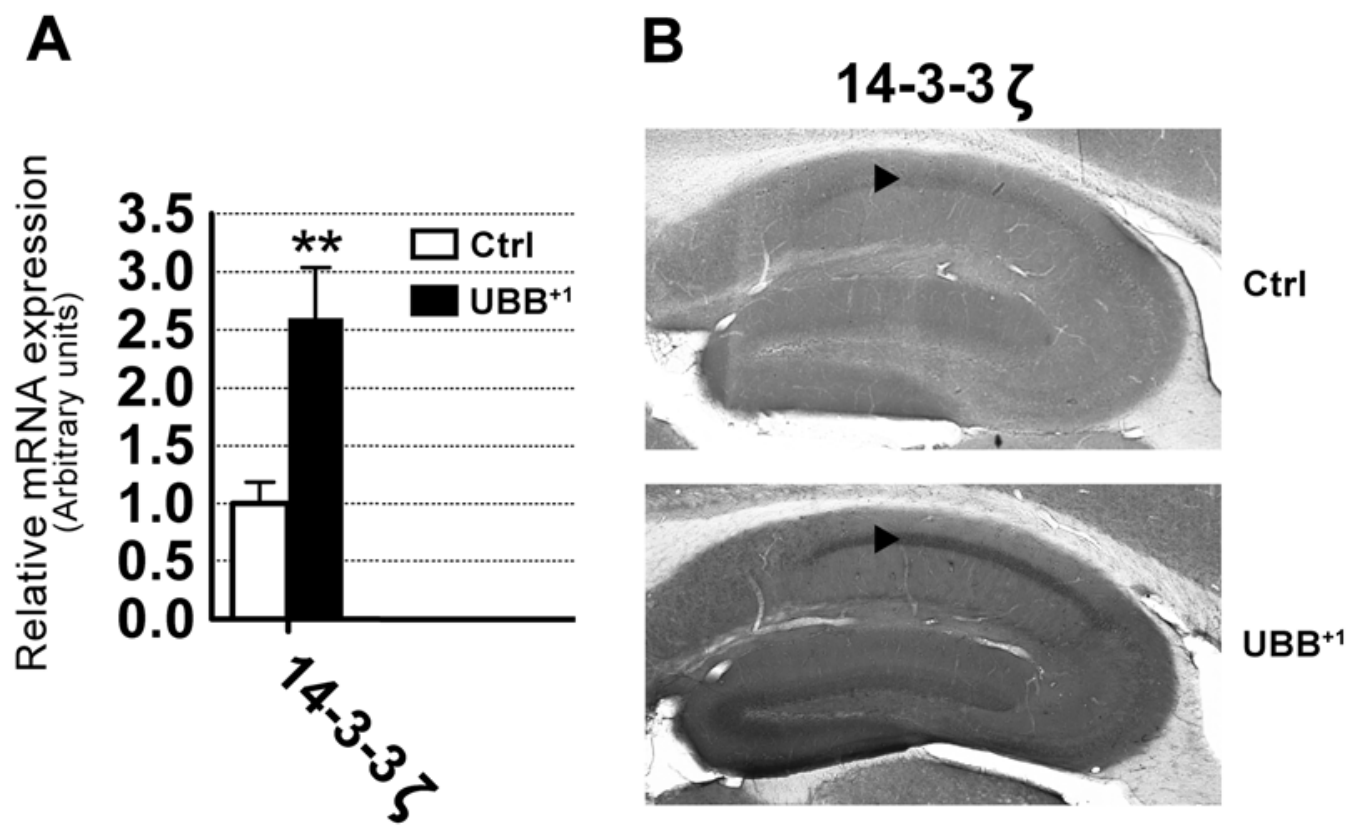

Figure 8: Synaptic plasticity marker 14-3-3 $\zeta$ expression $(A-B)$ in tg and control mice at the age of 9 months. mRNA profile (A) and protein expression by immunohistochemistry (B) of 14-3-3 $\zeta$. A) This gene is differentially regulated in the hippocampus of $\mathrm{UBB}^{+1} \mathrm{tg}$ mice versus age-matched controls. Note the significant increase in the mRNA expression for 14-3-3 $\zeta(p \leq 0.01)$. B) Increased protein expression of 14-3-3 $\zeta$ in the hippocampal CA1 region of $U B B^{+1}$ mice compared to control mice in $50 \mu m$ thick vibratome sections. Arrowheads indicate the pyramidal cell (e.g., CA1) region. Differences 
between strains were evaluated by the Mann-Whitney test. Error bars represent SEM ** $p<0.01$. This study was performed in collaboration with Prof. Dr. K. Marcus and Dr. T. Schulenborg, Ruhr University, Bochum, Germany.

As mentioned above, in the Supplementary data of Chapter 3 of the present thesis, the expression of a panel of genes in the hippocampal formation was studied by the use of qPCR. One of the significantly upregulated genes in the $\mathrm{UBB}^{+1}$ mice compared with the control mice was the $14-3-3 \zeta$ gene. This $14-3-3 \zeta$ gene showed an expression of $260 \%$ higher in the $\mathrm{UBB}^{+1}$ mice compared with the controls (Figure 8). Furthermore, the protein level of 14-3-3 $\zeta$ was analyzed by performing immunohistochemistry and 14-3-3 $\zeta$ was the only gene with consistent regulation on transcript and protein levels (Figure 8). An increased expression of the 14-3-3 $\zeta$ protein was demonstrated in the CA1 region of the hippocampus (Figure 8) and it was significantly upregulated in 22 months old $\mathrm{UBB}^{+1} \mathrm{tg}$ mice to $186 \%$ of normal levels (as determined by Mass Spec Phospho Proteome Analysis,, data not shown, in collaboration with Prof. Dr. K. Marcus) (Schulenborg et al., 2005). It is known that $U_{B B}^{+1}$ can induce heat shock proteins like of 14-3-3 $\zeta$; however, the molecular mechanisms behind this induction remains to be determined (Hope et al., 2003). These data showed a corresponding factor between AD and epilepsy.

A clear association exists between $A D$ and epileptic activity. Many patients suffering from AD have an increased risk for epileptic seizures and $10 \%$ to $22 \%$ will eventually develop unprovoked seizures (Mendez and Lim, 2003). Seizures appear most often in the temporal lobes carrying memory areas affected by AD pathology. Recently, a retrospective study showed that patients with amnestic mild cognitive impairment (aMCl) or $\mathrm{AD}$-associated epilepsy share some clinical features including early age of onset of cognitive decline, early incidence of seizures in the disease course, unilateral temporal epileptic foci detected by serial/extended electroencephalography (EEG) and transient cognitive dysfunction. This study suggests that epileptiform activity may be more prevalent in the early stages of $A D$ than previously assumed or may even accelerate $A D$ onset and that nonconvulsive epileptiform seizures can occur without diagnosis for years (Vossel et al., 2013). Hitherto, epileptic activity was commonly described in advanced AD stages caused by neuronal loss and gliosis (Romanelli et al., 1990). Beside general convulsive insults, dyscognitive focal seizures occur as well in AD patients, which may be convulsive or nonconvulsive which 
often results in altered consciousness, amnestic periods, confusion and difficulties in speaking. Because of the similarities in $A D$ and epilepsy symptoms, common molecular mechanisms underlying both diseases are suggested which makes it more difficult to examine the processes causing the memory deficits (Born, 2015).

AD patients suffering from familial AD with mutations in PS1, presenilin 2 (PS2) or APP and early-onset $A D$ demonstrate higher seizures rates compared to sporadic and lateonset cases. A $\beta$ and APP are seen as the possible mechanistic link between seizures and $A D$ based upon results of earlier studies showing the neurotoxicity of increased $A \beta$ levels and the negative feedback loop between $A \beta$ and neuronal activity (Born, 2015). Increased levels of APP have an important role in the development and maintenance of epileptiform activity. It was shown in APP/TTA (APP swe/Ind CamKIla) mice that the high SWD frequency could be normalized with suppression of the tg APP while this was not possible with an $\mathrm{Y}^{-}$ secretase inhibitor reducing $A \beta$ levels (Born, 2015). These data demonstrate the individual contributions of APP and A $\beta$ to seizure occurrence. More knowledge about the functioning of UPS system will possibly provide valuable targets to delay the AD pathogenesis and will increase the opportunities for therapeutic applications in the clinic.

\section{Outlook}

For a reliable functioning of the central nervous system, an efficient working protein degradation system is essential to guarantee the cellular homeostasis. All data mentioned above pointed to the fact that the mechanisms behind ubiquitin and UPS functioning are worthwhile to unravel further. Based on the phenotypes and on the results of the present thesis some suggestions for further research can be made.

A. In line with Chapter 2 and 3 we demonstrated that a comprehensive phenotypic screening of our $3413 \mathrm{tg}$ mouse line provided us a total view on each of the neuroanatomical distributions of $\mathrm{UBB}^{+1}$ of these mice and the results on a whole variation of executive functions. This screening taught us that these mice suffer from a respiratory phenotype and it is know from literature that AD patients also suffer from respiratory (Yaffe et al., 2011) and swallowing problems. Therefore, a total phenotypic screen of more transgenic animals would be worthwhile and would add probably more surprising data 
which will expand our knowledge about $A D$ and also for other related neurodegenerative diseases. A step further is testing AD patients individually for problems with respiration and swallowing. We started screening clinical reports of 121 controls and 276 AD patients (provided by the Dutch Brain Bank, Amsterdam) which were diagnosed for AD postmortem, for the presence of all kind of respiration problems and/or swallowing problems. Unfortunately, we could not find significant results but most probably this is due to the fact that there is less attention for these problems in hospitals and nursing homes and therefore these symptoms are presumably underdiagnosed. For future perspectives it would be worthwhile to screen stringently for these symptoms to come to a better diagnosis.

B. In line with Chapter 4 about the link between $\mathrm{UBB}^{+1}$ and mitochondrial damage, deciphering the ubiquitination process further in yeast strains will provide useful information. $\mathrm{UBB}^{+1}$ exists as variants, comprising ubiquitinated $\mathrm{UBB}^{+1}$, full-length $\mathrm{UBB}^{+1}$, and truncated $\mathrm{UBB}^{+1}$. All variants contribute to cytotoxicity, although ubiquitinated $\mathrm{UBB}^{+1}$ is more cytotoxic than full-length $\mathrm{UBB}^{+1}$ which is potentially more cytotoxic than truncated $\mathrm{UBB}^{+1}$. Thus, both the steady-state levels of $\mathrm{UBB}^{+1}$ and the proportional distribution among the $\mathrm{UBB}^{+1}$ variants dictates the cytotoxicity. The steady-state levels of $\mathrm{UBB}^{+1}$ are determined by proteasomal degradation, which potentially depends on the AAA-ATPases Cdc48/VCP and regulatory particle tripe (Rpt1-6). The proportional distribution among the $\mathrm{UBB}^{+1}$ variants depends on the activity of E3 ligases and DUBs. Therefore, we propose 3 new objectives to investigate in future experiments:

a. $\mathrm{UBB}^{+1}$ exists as ubiquitinated, full-length, and truncated variants (Dennissen et al., 2011). Although it is known that ubiquitinated $\mathrm{UBB}^{+1}$ demonstrates increased cytotoxicity as compared to full-length $\mathrm{UBB}^{+1}$, it remains elusive to which extent truncated $\mathrm{UBB}^{+1}$ contributes to cytotoxicity. For preventing the accumulation of lethal $\mathrm{UBB}^{+1}$, it is important to understand to which extent the $\mathrm{UBB}^{+1}$ variants contribute to cytotoxicity. Both the degradation of $\mathrm{UBB}^{+1}$ variants with higher lethality or their conversion into variants with lower lethality could prevent cell inactivity or cell loss. We anticipate confirming that ubiquitinated $\mathrm{UBB}^{+1}$ is more cytotoxic as compared to full-length $\mathrm{UBB}^{+1}$, and we will learn whether truncated $\mathrm{UBB}^{+1}$ is the less cytotoxic variant. 
b. We will determine whether the AAA-ATPases Cdc48/VCP and Rpt1-6 are involved in the degradation of $\mathrm{UBB}^{+1}$ in yeast and whether they influence its cytotoxicity. Whereas our previous work suggested a potential role of Cdc48/VCP in the degradation of $\mathrm{UBB}^{+1}$ (Chapter 4). The yeast proteasomal components Rpt1-6 demonstrate high homology to major UPS candidate genes discovered by the integrated gene expression and GWAS analysis of AD patients (IGAP, 2015). We anticipate to determine out whether $\mathrm{UBB}^{+1}$ is a substrate of Cdc48 (or Rpt1-6)dependent proteolysis. We expect to confirm our previous result that high Cdc48 activities protect from UBB ${ }^{+1}$-triggered cytotoxicity (Chapter 4), and we will learn whether this is true for high Rpt1-6 activities. VCP, the human homolog of Cdc48, will be tested for co-staining with $\mathrm{UBB}^{+1}$ in hippocampal and brainstem postmortem tissues from $A D$ patients and controls. If VCP co-exists with $U_{B B}^{+1}$, this would support that $\mathrm{UBB}^{+1}$ is a substrate of Cdc48/VCP. If VCP does not co-exist with $\mathrm{UBB}^{+1}$, this would favor a model in which VCP interrupts the lethal signaling cascade triggered by $\cup B B^{+1}$ at another level. The co-localization of $\mathrm{UBB}^{+1}$ and Rpt3 has been demonstrated (Zouambia et al., 2008). Consequently, the colocalization of Rpt3, UBB ${ }^{+1}$, and VCP, and the colocalization of Rpts with $\mathrm{UBB}^{+1}$ needs to be examined based on the results obtained in yeast.

c. We will address how DUB activities modulate the relative distribution among the $\mathrm{UBB}^{+1}$ variants and how they influence $\mathrm{UBB}^{+1}$-triggered cytotoxicity in yeast. 20 yeast genes encode DUBs (yeast genome database, SGD). We will include the analysis of the DUB Ubp6/USP14, which is inhibited by extended ubiquitins like $\mathrm{UBB}^{+1}$ in yeast (Krutauz et al., 2014), and the DUB Yuh1/UCH-L3, which truncates $\mathrm{UBB}^{+1}$ in yeast and mammalian cells (Dennissen et al., 2011).

d. Selected human homologs of yeast DUBs, which modify $\mathrm{UBB}^{+1}$ or modulate its cytotoxicity, need to be tested for co-staining with $\mathrm{UBB}^{+1}$ in hippocampal and brainstem postmortem tissues from $A D$ patients and controls. We anticipate discriminating between DUBs that directly interact with $\mathrm{UBB}^{+1}$, hinting at a role in modifying $\mathrm{UBB}^{+1}$ itself, and DUBs which modulate cytotoxicity at another level of the lethal signaling cascade triggered by $\mathrm{UBB}^{+1}$. Recently for example, it was shown that inhibitors of the DUB ubiquitin specific peptidase 14 (USP14) can 
enhance proteasome function in cells. Therefore, the development of strategies to increase the proteolytic capacity of the proteasome might be beneficial for disease progression (Lee et al., 2011;Schmidt and Finley, 2014). Therefore, drug development strategies resulting in an increase of the proteolytic capacity of the proteasome might slow down disease progression (e.g. small-molecule inhibitors of Usp14 activity as mentioned above).

C. In line with Chapter 5 and 6 we demonstrated that a decrease in $A \beta$ plaque load in our APPPS1/UBB ${ }^{+1}$ tg line is caused by $\mathrm{UBB}^{+1}$ accompanied by rises in $\gamma$-secretase activity/PS1 expression. In the current scientific paradigm it is thought that it is necessary to inhibit the $\mathrm{y}$-secretase activity as PS1 is responsible for the cleavage of APP C-terminal fraction (APP-CTF) and thereby creating $A \beta$ which will accumulate in $A \beta$ plaque load. However, until now treatments with $\gamma$-secretase inhibitors were so far unsuccessful (De Strooper, 2014). Therefore, based upon our results in the triple tg APPPS1/UBB ${ }^{+1}$ line, we suggest examining the effects of activation of $\gamma$-secretase activity. We anticipate finding lower number of $A \beta$ plaques which possibly will have an effect on behavioural read outs. Nevertheless, we could not show an improvement on contextual behaviour level. However, increasing $\mathrm{Y}$-secretase activity as early as possible in the AD pathology will perchance improve the memory dysfunction. In addition, we suggest as well reconsidering the detrimental role of $A \beta$ plaques in $A D$ pathology (Chen, 2015). Perhaps, the $A \beta$ oligomers are actually the fatal components in the cell

and the plaques are only a protective way of the cell to survive as long as possible.

D. From Chapters 2, 3, 5 and 6 seeding of misformed proteins (Jucker and Christen, 2013) caused by a dysfunctional UPS in the brainstem is a possibility that can be tested in the triple (APPPS1/UBB $\left.{ }^{+1}\right) \operatorname{tg}$ mice.

E. The data in Chapters 2, 3, 4, 5 and 6 confirmed recent studies (Manavalan et al., 2013; IGAP, 2015) that $\mathrm{UBB}^{+1}$ and proteasomal dysfunction has an important role in AD. As already mentioned by Chondrogianni et al., another treatment option is the activation of the proteasome and to test its effect on aging and longevity (Chondrogianni et al., 2015). The proteasome can be activated through genetic manipulation or through natural and 
chemical compounds. These aims can be further tested for example in our $3413 \mathrm{UBB}^{+1} \mathrm{tg}$ mice or the crossbreed of lines 85 and 3413 (APPPS1/UBB ${ }^{+1}$ ).

\section{General conclusion}

In the present thesis we used mouse brain tissue and yeast strains to perform experiments and the obtained results were subsequently validated in human post mortem tissue. Our anatomical and functional studies in the brains of line 3413 (Chapter 2, 3 and 4) showed its predictive power for $A D$ because for certain brain regions, the results of which could quite precisely be mirrored in the human brain. Also our APPPS1 and APPPS1/UBB ${ }^{+1}$ mice showed part of the pathology seen in $A D$ (Chapter 5 and 6 ). However, we have to realize that these and other $A D$ models have their limitations with respect to $A D$ which is a multifactorial disease. Recently, a meta-analysis showed that even among the best available animal models, conserved differences between mouse and human transcriptomes were found across multiple animal model versus human disease comparisons, even including aging (Burns et al., 2015). Mouse models show consistent trends towards several molecular functions like preserved mitochondrial function protein catabolism, DNA repair responses and chromatin maintenance. These data suggest that $A D$ and neurodegenerative diseases in general are even more complex with a multifactorial pathophysiology than current standard animal models can capture. Therefore, the value of animal models must not be overestimated and data originating from animal models of human disease needs to be evaluated critically each time. However, animal models together with bioinformatics are useful tools to unravel parts of the neurodegenerative puzzle.

While performing immunohistochemical experiments we also obtained anatomical data in the tg mouse model which could not be translated to the human brain. As an example, the topographic mapping of $\mathrm{UBB}^{+1}$ expression in tg line 3413 (Chapter 2), revealed a specific expression of $\mathrm{UBB}^{+1}$ in a subset of parasagittal stripes of Purkinje cells in the cerebellar cortex in these mice but we unable to find this expression pattern of $\mathrm{UBB}^{+1}$ in human cerebella. Immunohistochemistry and immunofluorescence reveals that $\mathrm{UBB}^{+1}$-positive cerebellar Purkinje neurons are always located within parasagittal stripes in cerebellar 
lobules VI, VII, IX and X as well as in the flocculus and paraflocculus (Figures 9-11). This expression pattern matches that of the constitutively expressed Purkinje cell antigen Hsp25 which is a small heat shock protein 25 (Hsp25) (Figure 11). Hsp25 is constitutively expressed in the central nervous system of rodents, notably in the cerebellum, brainstem, hypothalamus, and spinal cord (Armstrong et al., 2000). Selective expression of UBB ${ }^{+1}$ in a subpopulation of Hsp25-immunoreactive stripes indicates a functional relationship. Hsp25 is known to act as a molecular chaperone and has been specifically associated with the functioning of the UPS. Additionally, $\mathrm{UBB}^{+1}$ has been shown to induce chaperone expression in vitro (Hope et al., 2003). Hsp25 also possesses neuroprotective properties: Hsp25-positive Purkinje cells appear to be more resistant to cell death than Hsp25-negative Purkinje cells (Sarna and Hawkes, 2003). In addition to line 3413, 2 other strains of male $\mathrm{UBB}^{+1} \mathrm{tg}$ mouse lines (6663 and 8630) were examined for UBB ${ }^{+1}$ expression in the cerebellum. Line 6663 used the CamKinase Ila (CamKIla) promoter like tg line 3413 while in line 8630 the Thy 1,2 promoter was used. In tg line 6663 UBB $^{+1}$ immunoreactivity was present in the cerebellum as in line 3413 while in line 8630 cerebellar UBB ${ }^{+1}$ expression was absent. These data suggest that the expression of $\mathrm{UBB}^{+1}$ in the cerebellum might be promoter dependent. Endogenous $\mathrm{UBB}^{+1}$ could not be detected in post-mortem cerebella from $A D$ patients, probably due to the lack of NFTs in this brain area, which are highly associated with $\mathrm{UBB}^{+1}$ immunoreactivity (see Chapter 2). Even in AD patients with prominent neuropathology and a cerebellar phenotype (e.g. PS1 E280A Familial AD (FAD) patients with cerebellar ataxias), no $\mathrm{UBB}^{+1}$ accumulation or NFTs could be detected so far (Sepulveda-Falla et al., 2011). Therefore, the potential significance of this selective expression pattern of $\mathrm{UBB}^{+1}$ in the mouse cerebellum remains elusive, may be promoter dependent and underscores the differences between animal models and human diseases. 


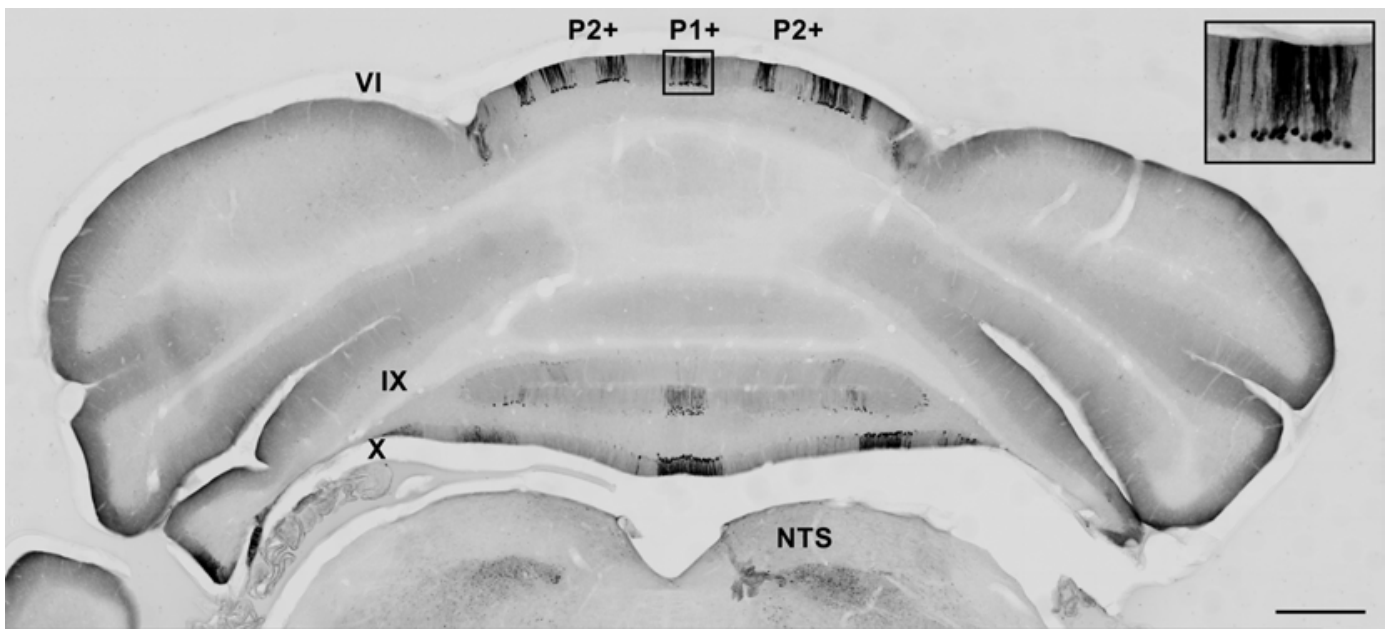

Figure 9: Expression of $U_{B B^{+1}}$ in the cerebellum of transgenic mouse line 3413 in a coronal section. Insert shows Purkinje cells. P\#: parasagittal stripe, VI/IX/X: lobule 6/9/10 of the cerebellum, NTS: nucleus of the solitary tract. Scale bar $=500 \mu \mathrm{m}$
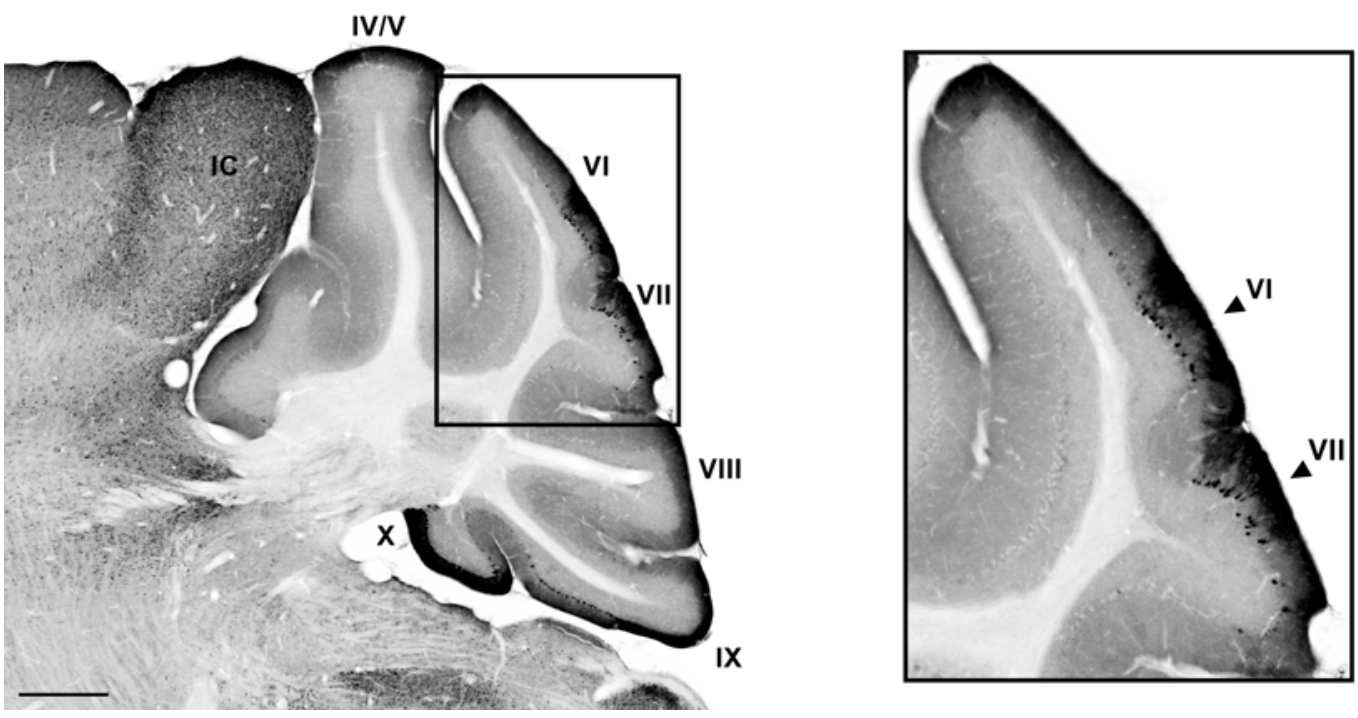

Figure 10: Expression of $U B B^{+1}$ in the cerebellum of a $U B B^{+1}$ tg mouse (line 3413) in a sagittal section. IVIVIVIVIIIVIII/IX/X: lobule 4/5/6/7/8/9/10 of the cerebellum, IC: inferior colliculus. These pictures were created with the help of Prof. Dr. David A. Hopkins and Drs. Mark B. Verheijen. Scale bar= $400 \mu \mathrm{m}$. 

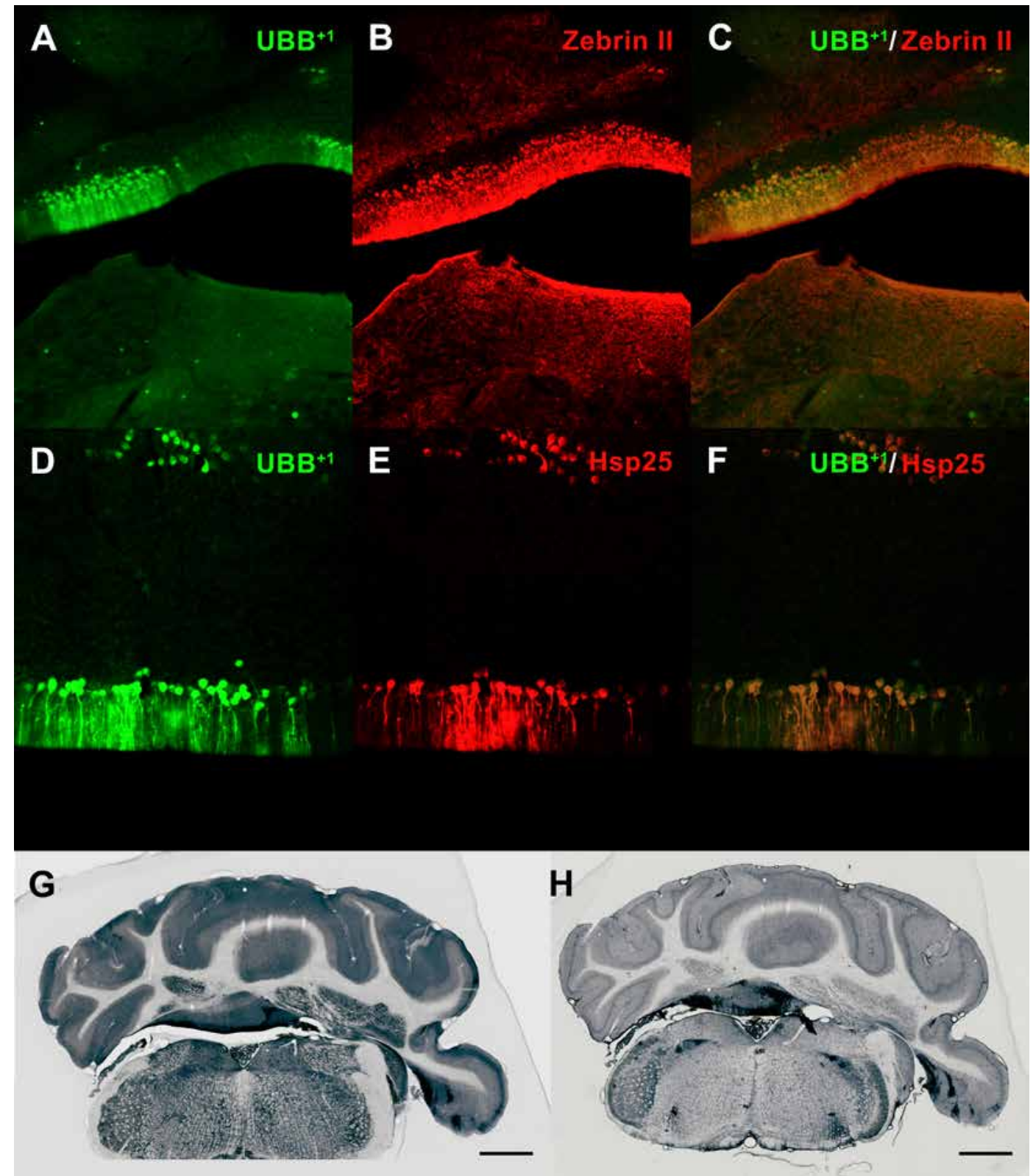

Figure 11: Expression of $U B B^{+1}(A)$ in a subset of zebrin II-positive stripes $(B)$ in the cerebellum of a $U B B^{+1}$-expressing tg mouse (line 3413) (A-C). C) demonstrate the merge between $(A)$ and $(B)$. $D-F$ ) $U B B^{+1}$-positive stripes (D) co-express heat shock protein 25 (Hsp25) (E). F) demonstrate the merge between (D) and (E). G-H) Coronal sections stained immunohistochemically with antibodies against 
$U_{B B}^{+1}(G)$ and Hsp25 (H). Note the expression of $U B B^{+1}$ and Hsp25 in the same cerebellar regions. Scale bars: $G-H=1 \mathrm{~mm}$

Several research questions regarding $A D$ and $\mathrm{UBB}^{+1}$ remain open. Nevertheless, the role of $\mathrm{UBB}^{+1}$ and a dysfunctional UPS is becoming more and more prominent in AD pathology and the present thesis aims to help the further unraveling of the hidden secrets of the UPS system. 


\section{References}

Armstrong, C.L., Krueger-Naug, A.M., Currie, R.W., and Hawkes, R. (2000). Constitutive expression of the 25-kDa heat shock protein Hsp25 reveals novel parasagittal bands of purkinje cells in the adult mouse cerebellar cortex. J Comp Neurol 416, 383-397.

Born, H.A. (2015). Seizures in Alzheimer's disease. Neuroscience 286, 251-263.

Brennan, G.P., Jimenez-Mateos, E.M., Mckiernan, R.C., Engel, T., Tzivion, G., and Henshall, D.C. (2013). Transgenic overexpression of 14-3-3 zeta protects hippocampus against endoplasmic reticulum stress and status epilepticus in vivo. PLoS One 8, e54491.

Burns, T.C., Li, M.D., Mehta, S., Awad, A.J., and Morgan, A.A. (2015). Mouse models rarely mimic the transcriptome of human neurodegenerative diseases: A systematic bioinformaticsbased critique of preclinical models. Eur J Pharmacol 759, 101-117.

Chen, M. (2015). The Maze of APP Processing in Alzheimer's Disease: Where Did We Go Wrong in Reasoning? Front Cell Neurosci 9, 186.

Chondrogianni, N., Voutetakis, K., Kapetanou, M., Delitsikou, V., Papaevgeniou, N., Sakellari, M., Lefaki, M., Filippopoulou, K., and Gonos, E.S. (2015). Proteasome activation: An innovative promising approach for delaying aging and retarding age-related diseases. Ageing Res Rev 23, 37-55.

Ciechanover, A. (2014). The complexity of recognition of ubiquitinated substrates by the $26 \mathrm{~S}$ proteasome. Biochim Biophys Acta 1843, 86-96.

De Strooper, B. (2014). Lessons from a failed gamma-secretase Alzheimer trial. Cell 159, 721-726.

Dennissen, F.J., Kholod, N., Hermes, D.J., Kemmerling, N., Steinbusch, H.W., Dantuma, N.P., and Van Leeuwen, F.W. (2011). Mutant ubiquitin $\left(\mathrm{UBB}^{+1}\right)$ associated with neurodegenerative disorders is hydrolyzed by ubiquitin C-terminal hydrolase L3 (UCH-L3). FEBS Lett 585, 2568-2574.

Garcia-Alloza, M., Robbins, E.M., Zhang-Nunes, S.X., Purcell, S.M., Betensky, R.A., Raju, S., Prada, C., Greenberg, S.M., Bacskai, B.J., and Frosch, M.P. (2006). Characterization of amyloid deposition in the APPswe/PS1dE9 mouse model of Alzheimer disease. Neurobiol Dis 24, 516-524.

Gregori, L., Fuchs, C., Figueiredo-Pereira, M.E., Van Nostrand, W.E., and Goldgaber, D. (1995). Amyloid beta-protein inhibits ubiquitin-dependent protein degradation in vitro. $\mathrm{J}$ Biol Chem 270, 19702-19708.

Hong, L., Huang, H.C., and Jiang, Z.F. (2014). Relationship between amyloid-beta and the ubiquitinproteasome system in Alzheimer's disease. Neurol Res 36, 276-282.

Hope, A.D., De Silva, R., Fischer, D.F., Hol, E.M., Van Leeuwen, F.W., and Lees, A.J. (2003). Alzheimer's associated variant ubiquitin causes inhibition of the $26 \mathrm{~S}$ proteasome and chaperone expression. Journal of Neurochemistry 86, 394-404.

Igap (2015). International Genomics of Alzheimer's Disease Consortium. Convergent genetic and expression data implicate immunity in Alzheimer's disease. Alzheimer's \& Dementia 11, 658-671.

Irmler, M., Gentier, R.J., Dennissen, F.J., Schulz, H., Bolle, I., Holter, S.M., Kallnik, M., Cheng, J.J., Klingenspor, M., Rozman, J., Ehrhardt, N., Hermes, D.J., Gailus-Durner, V., Fuchs, H., Hrabe De Angelis, M., Meyer, H.E., Hopkins, D.A., Van Leeuwen, F.W., and Beckers, J. 
(2012). Long-term proteasomal inhibition in transgenic mice by UBB(+1) expression results in dysfunction of central respiration control reminiscent of brainstem neuropathology in Alzheimer patients. Acta neuropathologica 124, 187-197.

Jucker, M., and Christen, Y. (2013). Proteopathic seeds and neurodegenerative diseases. Springer Heidelberg.

Jurisch-Yaksi, N., and Annaert, W. (2013). Protein quality control by Rer1p in the early secretory pathway: from mechanism to implication in Alzheimer's disease. Alzheimers Res Ther 5, 61.

Kageyama, S., Sou, Y.S., Uemura, T., Kametaka, S., Saito, T., Ishimura, R., Kouno, T., Bedford, L., Mayer, R.J., Lee, M.S., Yamamoto, M., Waguri, S., Tanaka, K., and Komatsu, M. (2014). Proteasome dysfunction activates autophagy and the Keap1-Nrf2 pathway. J Biol Chem 289, 24944-24955.

Kaneko, K., and Hachiya, N.S. (2006). The alternative role of 14-3-3 zeta as a sweeper of misfolded proteins in disease conditions. Med Hypotheses 67, 169-171.

Kaneko, M., Saito, R., Okuma, Y., and Nomura, Y. (2012). Possible involvement of ubiquitin ligase HRD1 insolubilization in amyloid beta generation. Biol Pharm Bull 35, 269-272.

Karam, R., Lou, C.H., Kroeger, H., Huang, L., Lin, J.H., and Wilkinson, M.F. (2015). The unfolded protein response is shaped by the NMD pathway. EMBO Rep 16, 599-609.

Keller, J.N., Hanni, K.B., and Markesbery, W.R. (2000). Impaired proteasome function in Alzheimer's disease. J Neurochem 75, 436-439.

Krutauz, D., Reis, N., Nakasone, M.A., Siman, P., Zhang, D., Kirkpatrick, D.S., Gygi, S.P., Brik, A., Fushman, D., and Glickman, M.H. (2014). Extended ubiquitin species are protein-based DUB inhibitors. Nat Chem Biol 10, 664-670.

Lee, M.J., Lee, B.H., Hanna, J., King, R.W., and Finley, D. (2011). Trimming of ubiquitin chains by proteasome-associated deubiquitinating enzymes. Mol Cell Proteomics 10, R110 003871.

Li, M., Wang, I.X., Li, Y., Bruzel, A., Richards, A.L., Toung, J.M., and Cheung, V.G. (2011). Widespread RNA and DNA sequence differences in the human transcriptome. Science 333, 53-58.

Lonskaya, I., Shekoyan, A.R., Hebron, M.L., Desforges, N., Algarzae, N.K., and Moussa, C.E. (2013). Diminished parkin solubility and co-localization with intraneuronal amyloid-beta are associated with autophagic defects in Alzheimer's disease. J Alzheimers Dis 33, 231-247.

Lopez Salon, M., Pasquini, L., Besio Moreno, M., Pasquini, J.M., and Soto, E. (2003). Relationship between beta-amyloid degradation and the 26S proteasome in neural cells. Exp Neurol 180, 131-143.

Maeda, T., Marutani, T., Zou, K., Araki, W., Tanabe, C., Yagishita, N., Yamano, Y., Amano, T., Michikawa, M., Nakajima, T., and Komano, H. (2009). An E3 ubiquitin ligase, Synoviolin, is involved in the degradation of immature nicastrin, and regulates the production of amyloid beta-protein. FEBS J 276, 5832-5840.

Manavalan, A., Mishra, M., Feng, L., Sze, S.K., Akatsu, H., and Heese, K. (2013). Brain site-specific proteome changes in aging-related dementia. Experimental \& Molecular Medicine 45, e39.

Maslow, A.H. (1943). A Theory of Human Motivation. Psychological Review 50, 370-396.

Mendez, M., and Lim, G. (2003). Seizures in elderly patients with dementia: epidemiology and management. Drugs Aging 20, 791-803. 
Minkeviciene, R., Rheims, S., Dobszay, M.B., Zilberter, M., Hartikainen, J., Fulop, L., Penke, B., Zilberter, Y., Harkany, T., Pitkanen, A., and Tanila, H. (2009). Amyloid beta-induced neuronal hyperexcitability triggers progressive epilepsy. J Neurosci 29, 3453-3462.

Mucke, L., and Selkoe, D.J. (2012). Neurotoxicity of amyloid beta-protein: synaptic and network dysfunction. Cold Spring Harb Perspect Med 2, a006338.

Park, H.J., Shabashvili, D., Nekorchuk, M.D., Shyqyriu, E., Jung, J.I., Ladd, T.B., Moore, B.D., Felsenstein, K.M., Golde, T.E., and Kim, S.H. (2012). Retention in endoplasmic reticulum 1 (RER1) modulates amyloid-beta (Abeta) production by altering trafficking of gammasecretase and amyloid precursor protein (APP). J Biol Chem 287, 40629-40640.

Perry, G., Friedman, R., Shaw, G., and Chau, V. (1987). Ubiquitin is detected in neurofibrillary tangles and senile plaque neurites of Alzheimer disease brains. Proc Natl Acad Sci U S A 84, 30333036.

Petry, F.R., Pelletier, J., Bretteville, A., Morin, F., Calon, F., Hebert, S.S., Whittington, R.A., and Planel, E. (2014). Specificity of anti-tau antibodies when analyzing mice models of Alzheimer's disease: problems and solutions. PLoS One 9, e94251.

Romanelli, M.F., Morris, J.C., Ashkin, K., and Coben, L.A. (1990). Advanced Alzheimer's disease is a risk factor for late-onset seizures. Arch Neurol 47, 847-850.

Ryu, H.W., Park, C.W., and Ryu, K.Y. (2014). Disruption of polyubiquitin gene Ubb causes dysregulation of neural stem cell differentiation with premature gliogenesis. Sci Rep 4, 7026.

Sarna, J.R., and Hawkes, R. (2003). Patterned Purkinje cell death in the cerebellum. Prog Neurobiol 70, 473-507.

Schmidt, M., and Finley, D. (2014). Regulation of proteasome activity in health and disease. Biochim Biophys Acta 1843, 13-25.

Schulenborg, T., Fischer, D.F., H.E., M., Van Leeuwen, F.W., and Marcus, K. (2005). "Phospho proteome analysis of an Alzheimer's disease transgenic mouse model $\left(\mathrm{UBB}^{+1}\right)$ using a $\mathrm{Q}$ Trap Instrument", in: American Society for Mass Spectrometry. (San Antonio, Texas).

Sepulveda-Falla, D., Matschke, J., Bernreuther, C., Hagel, C., Puig, B., Villegas, A., Garcia, G., Zea, J., Gomez-Mancilla, B., Ferrer, I., Lopera, F., and Glatzel, M. (2011). Deposition of hyperphosphorylated tau in cerebellum of PS1 E280A Alzheimer's disease. Brain Pathol 21, 452-463.

Tan, Z., Sun, X., Hou, F.S., Oh, H.W., Hilgenberg, L.G., Hol, E.M., Van Leeuwen, F.W., Smith, M.A., O'dowd, D.K., and Schreiber, S.S. (2007). Mutant ubiquitin found in Alzheimer's disease causes neuritic beading of mitochondria in association with neuronal degeneration. Cell Death Differ 14, 1721-1732.

Tanabe, C., Maeda, T., Zou, K., Liu, J., Liu, S., Nakajima, T., and Komano, H. (2012). The ubiquitin ligase synoviolin up-regulates amyloid beta production by targeting a negative regulator of gamma-secretase, Rer1, for degradation. J Biol Chem 287, 44203-44211.

Tseng, B.P., Green, K.N., Chan, J.L., Blurton-Jones, M., and Laferla, F.M. (2008). Abeta inhibits the proteasome and enhances amyloid and tau accumulation. Neurobiol Aging 29, 1607-1618.

Van Leeuwen, F.W., Van Tijn, P., Sonnemans, M.A., Hobo, B., Mann, D.M., Van Broeckhoven, C., Kumar-Singh, S., Cras, P., Leuba, G., Savioz, A., Maat-Schieman, M.L., Yamaguchi, H., Kros, J.M., Kamphorst, W., Hol, E.M., De Vos, R.A., and Fischer, D.F. (2006). Frameshift 
proteins in autosomal dominant forms of Alzheimer disease and other tauopathies. Neurology 66, S86-92.

Van Tijn, P., De Vrij, F.M., Schuurman, K.G., Dantuma, N.P., Fischer, D.F., Van Leeuwen, F.W., and Hol, E.M. (2007). Dose-dependent inhibition of proteasome activity by a mutant ubiquitin associated with neurodegenerative disease. Journal of Cell Science 120, 1615-1623.

Viola, K.L., and Klein, W.L. (2015). Amyloid beta oligomers in Alzheimer's disease pathogenesis, treatment, and diagnosis. Acta Neuropathol 129, 183-206.

Vossel, K.A., Beagle, A.J., Rabinovici, G.D., Shu, H., Lee, S.E., Naasan, G., Hegde, M., Cornes, S.B., Henry, M.L., Nelson, A.B., Seeley, W.W., Geschwind, M.D., Gorno-Tempini, M.L., Shih, T., Kirsch, H.E., Garcia, P.A., Miller, B.L., and Mucke, L. (2013). Seizures and epileptiform activity in the early stages of Alzheimer disease. JAMA Neurol 70, 1158-1166.

Wang, D. (2008). Discrepancy between mRNA and protein abundance: insight from information retrieval process in computers. Comput Biol Chem 32, 462-468.

Wang, I.X., Core, L.J., Kwak, H., Brady, L., Bruzel, A., Mcdaniel, L., Richards, A.L., Wu, M., Grunseich, C., Lis, J.T., and Cheung, V.G. (2014). RNA-DNA differences are generated in human cells within seconds after RNA exits polymerase II. Cell Rep 6, 906-915.

Yaffe, K., Laffan, A.M., Harrison, S.L., Redline, S., Spira, A.P., Ensrud, K.E., Ancoli-Israel, S., and Stone, K.L. (2011). Sleep-disordered breathing, hypoxia, and risk of mild cognitive impairment and dementia in older women. JAMA 306, 613-619.

Zhang, M., Cai, F., Zhang, S., Zhang, S., and Song, W. (2014). Overexpression of ubiquitin carboxylterminal hydrolase L1 (UCHL1) delays Alzheimer's progression in vivo. Sci Rep 4, 7298.

Zhao, X., and Yang, J. (2010). Amyloid-beta peptide is a substrate of the human 20S proteasome. ACS Chem Neurosci 1, 655-660.

Zheng, C., Geetha, T., and Babu, J.R. (2014). Failure of ubiquitin proteasome system: risk for neurodegenerative diseases. Neurodegener Dis 14, 161-175.

Zouambia, M., Fischer, D.F., Hobo, B., De Vos, R.A., Hol, E.M., Varndell, I.M., Sheppard, P.W., and Van Leeuwen, F.W. (2008). Proteasome subunit proteins and neuropathology in tauopathies and synucleinopathies: Consequences for proteomic analyses. Proteomics 8, 1221-1236. 

Summary 
Homeostasis of protein turnover (synthesis and degradation) is essential for cells to survive. Each day 5-8\% of all proteins are metabolized as part of processes such as neuronal development and synaptic plasticity. This turnover requires efficient protein quality control for which the ubiquitin-proteasome system (UPS) and autophagy-lysosome pathway are mainly responsible. Accumulation of different types of protein aggregates is a characteristic of most age-related neurodegenerative diseases (e.g. Alzheimer's disease, AD). Recent pooled GWAS and proteome studies identified the UPS as a key regulator in AD. Molecular misreading is a mechanism where the inaccurate conversion of genomic DNA results in dinucleotide deletions in simple monotonic base sequences in RNA causing "+1 proteins"(e.g. ubiquitin $\mathrm{B}^{+1}, \mathrm{UBB}^{+1}$ ). UBB ${ }^{+1}$ accumulates specifically in the hallmarks of all types of Alzheimer's disease (AD), other tauopathies, and in polyglutamine diseases but not in synucleinopathies. This suggests that +1 proteins may be involved in these neurodegenerative diseases. Ubiquitin is normally tagged, via its C-terminal glycine residue, to a degron within a target protein that undergoes subsequent degradation by the 26S proteasome. $\mathrm{UBB}^{+1}$ lacks the C-terminal glycine residue and has 20 amino acids added to its C-terminus. Consequently, $\mathrm{UBB}^{+1}$ cannot ubiquitinate, is ubiquitinated itself, is refractory to deubiquitination, inhibits the UPS dose-dependently, and may impair the functioning of the $26 \mathrm{~S}$ proteasome resulting in impairment of mitochondrial functioning. The $\mathrm{UBB}^{+1}$ extension is just a few amino acids too short to be efficiently degraded by the proteasome. This provides an explanation for the accumulation of $U_{B B}{ }^{+1}$ in the hallmarks of tauopathies and several polyglutamine diseases.

Chapter 1 is the general introduction of this thesis and considers not only the major hypotheses of $A D$ but also focusses especially on proteasomal dysfunction by $U_{B B}^{+1}$ in combination with oxidative stress which contribute to disease-specific inclusions.

Chapter 2 presents a comprehensive topographic mapping of $\mathrm{UBB}^{+1}$ distribution in $\mathrm{tg}$ mouse line \#3413 throughout the brain. Most studies have concentrated primarily on the cortical areas and the hippocampus. Our topographic screen identified a collection of additional regions immunoreactive for $\mathrm{UBB}^{+1}$ in line 3413. We focused on five brain regions, namely olfactory area, inferior colliculus, raphe nuclei, basal ganglia and nucleus basalis of Meynert, which are known to be affected in AD. We demonstrated that $\mathrm{UBB}^{+1}$ distribution 
in these regions in tg line 3413 is partly mirrored in the AD brain. Specifically, in human brain areas with substantial accumulation of tangle-bearing neurons, such as nucleus basalis of Meynert and raphe nuclei $\mathrm{UBB}^{+1}$ accumulation was present. These new findings can be used to disclose new avenues for research about the impact of proteasomal stress on $\mathrm{AD}$ but also on other multifactorial tauopathies and polyglutamine diseases.

Chapter 3 is an extension of the topographic $U_{B B}^{+1}$ mapping pointing to the presence of $\mathrm{UBB}^{+1}$ in the nucleus of the solitary tract and the nucleus parabrachialis in transgenic line 3413. Both are brainstem regions involved in controlling respiration. This expression pattern in the mice has been linked to functional changes, such as changes in spontaneous breathing pattern and an altered hypoxic response. To validate our findings, $\mathrm{UBB}^{+1}$ distribution was examined in the same human brainstem regions as well. The human brainstem screen showed the presence of $\mathrm{UBB}^{+1}$ in neurofibrillary tangles as well which suggests that it would be useful to screen patients with mild cognitive impairments for respiratory changes.

Chapter 4 describes a yeast model used to unravel the detrimental effects of $\mathrm{UBB}^{+1}$ on cell death mechanisms regulated by mitochondria. $U_{B B}^{+1}$ was responsible for an enhancement of the basic amino acids arginine, ornithine and lysine and this event is seen as a toxic event which can be reversed by the mitochondrial-mediated proteolysis by CdC48/Vms1 proteins. These data were strengthened by validating the expression of $\mathrm{Vms} 1$ and $\mathrm{UBB}^{+1}$ in the $A D$ brain hippocampus by immunohistochemistry showing co-localization of these proteins and aberrant tau. The protective mechanisms of Vms1 have potentially farreaching pathophysiological implications.

In chapter 5, we examined in tg mice the effect of combining two pathologies described in $A D$ : $U B B^{+1}$ expression and $A \beta$ plaque accumulation. A remarkable decrease was found in $A \beta$ plaque load and $A \beta_{42}$ levels in APPPS1/UBB ${ }^{+1}$ mice. These findings were a surprise as we expected an increase in $A \beta$ plaque load in an environment of proteasomal stress.

In Chapter 6, the molecular mechanisms behind the decrease in $A \beta$ plaque load due to proteasomal inhibition by $\mathrm{UBB}^{+1}$ and the effect on cognitive behavior was examined in the transgenic APPPS1 mouse line and the crossbreed with line 3413; APPPS1/UBB ${ }^{+1}$. Surprisingly, a decreased $\mathrm{y}$-secretase activity, a flawed PS1 expression and worsened 
cognitive behavior was shown in APPPS1 mice, which is in contrast to most of the literature on $\mathrm{Y}$-secretase activity in AD. In the APPPS1/UBB ${ }^{+1}$ mice an even worse behavioral phenotype was observed with less $A \beta$ plaque load and a restored $\gamma$-secretase activity compared with the APPPS1 mice. These data forces researchers to reconsider the role of $A \beta$ plaques in $A D$ : detrimental or protective? , and secondly, the benefit of $\gamma$-secretase inhibitor trials.

Finally, chapter 7 provided a general discussion of the data presented in this thesis and suggestions for future research were delineated. 


\section{Samenvatting}


Homeostase tussen eiwitsynthese en eiwitdegradatie is essentieel voor cellen in een organisme om te overleven. Elke dag worden 5-8\% van alle eiwitten gemetaboliseerd als onderdeel van verschillende processen zoals neuronale ontwikkeling en synaptische plasticiteit. Deze omzetting vereist efficiënte mechanismen voor kwaliteitscontrole van de eiwitten. Het ubiquitine-proteasome (UPS) systeem en het autophagie-lysosoom systeem zijn de twee belangrijkste mechanismen verantwoordelijk voor de kwaliteitscontrole. De accumulatie van eiwit aggregaten is een kenmerk van de meeste leeftijds-gerelateerde neurodegeneratieve aandoeningen (bv. De ziekte van Alzheimer (AD)). Recent hebben gepoolde GWAS en proteoom studies het UPS aangeduid als een van de sleutelcomponenten in AD. In eenvoudige monotone RNA sekwenties komen dinucleotide deleties voor die resulteren in een fenomeen dat moleculaire leesfout genoemd wordt. Dit fenomeen is een mechanisme dat veroorzaakt wordt door een onnauwkeurige omzetting van het genomische DNA. Deze verschuiving in het leesraam veroorzaakt "+1 eiwitten" (bv. Ubiquitin $\mathrm{B}^{+1}, \mathrm{UBB}^{+1}$ ). $\mathrm{UBB}^{+1}$ stapelt zich specifiek op in de kenmerken van $A D$, in tauopathies en in polyglutamine aandoeningen maar niet in synucleinopathies. Dit wijst erop dat +1 protein betrokken zijn in neurodegeneratieve ziekten. Ubiquitine is normaal via zijn C-terminale glycine verbonden met een degron in een target eiwit. Dit gelabelde eiwit wordt vervolgens gedegradeerd door het $26 \mathrm{~S}$ proteasoom complex. $\mathrm{UBB}^{+1}$ heeft 20 aminozuren extra aan zijn C-terminus en mist hierdoor de C-terminale glycine. Hierdoor kan het $\mathrm{UBB}^{+1}$ ewit niet ubiquitineren, niet ge-ubiquitineerd worden, is het weerspanning tegen de-ubiquitinatie, veroorzaakt het dosis-afhankelijke inhibitie van het UPS en beschadigt het de functie van het $26 \mathrm{~S}$ proteasome wat resulteert in het aantasten van de mitochondriale werking. De C-terminale $\mathrm{UBB}^{+1}$ extensie is net enkele aminozuren te kort om efficiënt gedegradeerd te worden door het proteasoom. Dit verklaart de accumulatie van $\mathrm{UBB}^{+1}$ in de kenmerken van tauopathies en in verschillende polyglutamine ziekten.

Hoofdstuk 1 is de algemene introductie van deze thesis en deze focust op de belangrijkste theorieën van $A D$ maar ook voornamelijk op proteasomale dysfunction door $\mathrm{UBB}^{+1}$ in combinatie met oxidatieve stress. Beide factoren dragen bij aan ziekte-gerelateerde inclusies. 
Hoofdstuk 2 beschrijft een uitgebreide topografische mapping van de $U_{B B}^{+1}$ verdeling in het brein van $3413 \mathrm{tg}$ muizen. Tot nog toe hebben de studies zich vooral geconcentreerd op corticale gebieden en de hippocampus. Deze topografische screen heeft tal van additionele $U_{B B}^{+1}$ immunoreactieve regio's geïdentificeerd. Wij hebben gefocust op 5 hersengebieden: de olfactoire regio, de inferior colliculus, de raphe nuclei, de basale ganglie en de nucleus basalis van Meynert. Van deze regio's is al eerder aangetoond dat ze aangetast zijn in $A D$ patiënten. We konden aantonen dat de verdeling van $U_{B B}^{+1}$ in deze regio's in tg line 3413 gedeeltelijk gespiegeld worden in het humane AD brein. Dit voornamelijk in humane hersenregio's die een substantiële accumulatie van tangles vertonen in hun neuronen in gebieden zoals de nucleus basalis of Meynert en de raphe nuclei. Deze bevindingen kunnen gebruikt worden om nieuwe wegen te ontdekken voor onderzoek over de impact van proteasomale stress op $A D$, maar ook op andere multifactoriële aandoeningen zoals tauopathies en polyglutamine aandoeningen.

Hoofdstuk 3 is een feite een extensie van de topografische $U_{B B}^{+1}$ mapping. Het wijst op de duidelijke aanwezigheid van $\mathrm{UBB}^{+1}$ in de nucleus tractus solitarius en de nucleus parabrachialis in de transgenen 3413 lijn. Beide nuclei behoren tot de hersenstam en zijn betrokken in de controle van de ademhaling. De expressiepatroon van $\mathrm{UBB}^{+1}$ in de hersenstam deze muizen hebben we kunnen koppelen aan functionele veranderingen. $\mathrm{Er}$ worden afwijkingen in het spontaan ademhalingspatroon en in de respons op hypoxia. Om onze bevindingen in de muis te valideren, zijn we een stapje verder gegaan en hebben we dezelfde hersenstam gebieden in de mens onderzocht. Deze screen toonde ook de aanwezigheid van $\mathrm{UBB}^{+1}$ aan in de neurofibrillary tangles in de humane hersenstam. Deze data tonen aan dat het nuttig kan zijn om kleine ademhalingsverandering in patiënten met milde cognitieve stoornis te registreren en te onderzoeken.

In hoofdstuk 4 is een gistmodel gebruikt om de schadelijke effecten van UBB ${ }^{+1}$ op celdood mechanismen, die gereguleerd worden door mitochondria, te ontrafelen. $\mathrm{UBB}^{+1}$ is verantwoordelijk voor een verhoging in fundamentele aminozuren arginine, ornithine en lysine in de mitochondria. Dit proces wordt gezien als de toxische oorzaak dat omgekeerd kan worden door mitochondriale gemedieërde proteolysis door CdC48/Vms1 eiwitten. Deze bevindingen werden nog versterkt door het aantonen van co-lokalisatie van Vms1 en 
$\mathrm{UBB}^{+1}$ alsook tau in het humane brein. De protectieve mechanismen van Vms1 kan potentieel verstrekkende pathofysiologische gevolgen veroorzaken.

Hoofdstuk 5 beschrijft het effect van het combineren van twee pathologieën beschreven in $A D$ met name: $U_{B B}{ }^{+1}$ expressie en $A \beta$ plaques. Een significante verlaging van $A \beta$ plaques en $A \beta_{42}$ was aangetoond in APPPS1/UBB ${ }^{+1}$ muizen. Deze bevindingen waren een verassing aangezien we een verhoging van $A \beta$ plaques verwachtten in een omgeving van proteasomale stress.

Hoofdstuk 6 had als doel om de moleculare mechanismen achter de $A \beta$ plaque verlaging door proteasomale inhibitie te ontrafelen en om het effect ervan op cognitief gedrag te onderzoeken in de transgene APPPS1 muislijn en in de kruising met lijn 3413. Een verlaagde $\mathrm{Y}$-secretase activiteit en PS1 expressie en verslechterd cognitief gedrag was aangetoond in APPPS1 muizen. Dit is in contrast met de meeste literatuur over de $\mathrm{Y}^{-}$ secretase activiteit in AD. In de APPPS1/UBB ${ }^{+1}$ muizen hebben we een nog slechter gedragsfenotype vastgesteld met minder $A \beta$ plaque load en een herstelde $\gamma$-secretase activiteit vergeleken met de APPPS1 dieren. Deze data dwingt onderzoekers er toe om opnieuw na te denken of de exacte rol van $A \beta$ plaques in $A D$ : schadelijk of protectief? alsook over het voordeel van $\mathrm{y}$-secretase inhibitor studies.

Tot slot, hoofdstuk 7 beschrijft een algemene discussie de data gepresenteerd in deze thesis en suggesties voor toekomstig onderzoek. 
Valorization 


\section{Societal relevance}

Alzheimer's disease (AD) is a progressive neurodegenerative disorder and the most common cause of dementia accounting for $60-80 \%$ of all dementia patients (Barnes and Yaffe, 2011). It is a very complex disease because of its multifactorial nature and it is characterized by a decline in cognition, language, planning and solving skills, as well as behavioral changes affecting a person's everyday activities. AD patients in the final stage of the disease are bedridden and the disease is ultimately fatal. Currently, there are over 5 million Americans suffering from AD while in The Netherlands, around 140.000 people are currently suffering from this disease. These numbers are expected to triple around 2050. $A D$ is the $6^{\text {th }}$ leading cause of death and it kills more people than breast and prostate cancer combined. It is a devastating disease which cannot be slowed, stopped or prevented. Between 2000 and 2013 deaths of other diseases (including heart disease, stroke, breast and prostate cancer, and HIVIAIDS) decreased, while AD deaths increased $71 \%$ in the USA. It causes an enormous economic burden, and in 2015 the disease will cost the US nation $\$ 226$ billion and an amount that is estimated to increase to $\$ 1.1$ trillion by 2050 . By 2030, 72 million baby boomers will reach the age to be at greater risk for developing AD. Less than $50 \%$ of people with $A D$ are being told of their diagnosis (Alzheimer's Association 2015). These data together illustrate the impact of this tragic disease on society. Researchers have a pivotal task to make early detection possible, to find a way to slow down the disease progression, and to unravel the cause(s) and the molecular mechanisms behind it to come with a drug or a therapy to prevent and/or reverse the disease. The goal of the present thesis was to help unraveling the molecular mechanisms behind the disease.

\section{Diagnosis of $A D$}

Diagnosing $A D$ is difficult as no simple test is available and is most commonly made by a general practioner. A wide variety of tools and lists are present to diagnose the disease. Making the diagnosis is usually based on the medical anamnesis and family history, information from a person of the intimate circle of the patient, cognitive tests, DSM-V criteria and neurological examinations and MRI (Alzheimer's Association 2015). A definite diagnosis of $A D$ is only possible postmortemly after macro- and microscopic examination 
of the brain based on brain atrophy and the presence of extracellular plaques and neurofibrillary tangles in the brain. Researchers believe that early detection will be key to preventing, slowing and stopping AD. Therefore, in the last decade researchers have spent much effort in research on early detection of AD. Research has been performed on the selection of suitable biomarkers for the diagnosis for AD. One of these are the biomarkers showing the level of $A \beta$ accumulation in the blood or cerebrospinal fluid (CSF). Other research groups are examining the comorbidities of $A D$ :

a. Olfactory dysfunction is associated with aging, represented by a progressive decline in the ability to detect, identify and discriminate odors (Mobley et al., 2014). Olfactory deficits have been described in $A D$ as well and it is a predictor of the incidence of mild cognitive impairment $(\mathrm{MCl})$ and of the conversion of $\mathrm{MCl}$ to $\mathrm{AD}$ (Djordjevic et al., 2008;Attems et al., 2014).

b. Age-related auditory deficits are also quite common in the elderly. It has been shown that structural changes are present in the central auditory pathways of $A D$ patients (Sinha et al., 1993). An increased risk of AD is present in individuals with central auditory dysfunction (CAD) and it is suggested that CAD is an early manifestation of $A D$ that occurs before any sign of cognitive decline. Therefore, auditory dysfunction may be a valuable tool to diagnose AD at an early stage.

c. An important comorbidity of $A D$ is depression which may precede the clinical symptoms of AD by several years (Sierksma et al., 2010). However, it is unclear whether depression is a risk factor (Geerlings et al., 2008) or a prodromal sign (Wilson et al., 2004) for dementia and AD.

d. The basal ganglia including a network of dopaminergic subunits, are essential in the reward and reinforcement mechanisms and in regulating emotional behaviour (de Jong et al., 2011), as well as motor functions. Feelings of apathy have been noted in $A D$ patients which correlated with the detection of dopaminergic dysfunction. Apathy was ascribed in $47 \%$ of patients with mild AD and increased event to $80 \%$ in patients with severe AD (Mitchell et al., 2011).

e. Respiratory problems and swallowing impairments are common in AD patients and frequently result in aspiration pneumonia. It is suggested that changes in cortical control of swallowing may begin long before dysphagia becomes apparent 
(Humbert et al., 2010). These basic functions are regulated by the nucleus of the solitary tract (NTS) and the parabrachial nucleus (PBN) in the brainstem. The present thesis showed changes in spontaneous breathing patterns and altered hypoxic response in $\mathrm{UBB}^{+1} \operatorname{tg}$ mice (an $\mathrm{AD}$ mouse model), suggesting a central dysfunction of respiratory regulation.

It would be worthwhile to take possible comorbidities of $A D$ into account in order to come earlier to a more accurate diagnosis of $A D$ as some are present long before any sign of cognitive dysfunction. The present thesis demonstrated the accumulation of $\mathrm{UBB}^{+1}$ in an AD tg mouse model (line 3413), pointing to the involvement of a dysfunctional UPS in AD. For some anatomical regions (NTS, PBN, raphe nuclei and nucleus basalis of Meynert (NBM)) we showed $\mathrm{UBB}^{+1}$ accumulation in the same regions in the human brain compared with the mouse brain. Besides this, we also examined the accumulation of plaques and tangles in olfactory bulb (OB), inferior colliculus (IC), basal ganglia and raphe nuclei and the respiratory brain stem areas. We confirmed earlier studies and showed the presence of these hallmarks of pathology in the different anatomical regions.

As $A D$ is a multifactorial disease, researchers have to take every possible causative factor into account to come to a therapy or an early diagnosis of this disease. The present thesis expanded knowledge about $\mathrm{UBB}^{+1}$ accumulation in the tg mouse brain as well as in the human brain. We emphasized the role of $\mathrm{UBB}^{+1}$ in different comorbidities of $A D$ and concluded that comorbidities can be very pivotal to come to an early diagnosis of this neurodegenerative disease. The earlier the diagnosis of $A D$ the lower the health care costs and the higher the quality of life are for the patient and family. 


\section{Possible therapeutic applications}

Currently, no effective pharmacological drug or therapy is available to prevent, stop or cure $A D$ because of the complexity of the disease. Future perspectives are dramatic as the total number of patients will increase enormously and a solution for this neurodegenerative disease is necessary. It is a race against time for researchers to come with a therapy. Several treatment options are used today in the clinic today like cholinesterase inhibitors. These inhibitors prevent the breakdown of the neurotransmitter acetycholine which is pivotal for learning and memory. Unfortunately, it only delays worsening of symptoms for 6 to 12 months on average for around $50 \%$ of patients taking these inhibitors. An NMDA receptor antagonist which regulates the activity of another neurotransmitter involved in learning and memory called glutamate is currently also given to $A D$ patient. It retards the progression of the symptoms temporarily for some $A D$ patients. In addition to date, immunization trials in AD patients have not been effective in terms of ameliorating or curing dementia. Probably these trials started too late in the disease progression (Lannfelt et al., 2014). Removal of $A \beta$ in $A D$ brains has been questioned because studies show that the amount and distribution of $A \beta$ accumulation do not correlate with the degree of cognitive impairment and in addition $A \beta$ is present as well in the brains of cognitively normal elderly people (Drachman, 2014). It has been shown that immunisation with $A \beta_{42}$ resulted in clearance of amyloid plaques in patients with $A D$ but this clearance did not prevent progressive neurodegeneration (Holmes et al., 2008). The use of $y$-secretase inhibitors were unsuccessful as well so far, as the clinical trials were disappointing, possibly due to lack of knowledge of their working mechanism (De Strooper, 2014).

After several failures in developing $A D$ therapies, other aspects, which potentially contribute to $A D$ progression, come into focus for the development of therapeutics. Recent data suggest that a dysfunctional UPS is an early and causative event for AD progression, and a prime target for AD therapeutics (Manavalan et al., 2013;IGAP, 2015). The present thesis elaborates new insights in the genesis of the disease considering this aspect. Preventing the accumulation of the AD-associated $\mathrm{UBB}^{+1}$, which impairs the UPS at high levels, could be a promising approach for promoting neuronal survival, thereby delaying the progression of $A D$. Chapter 6 shows that UPS impairment upon $\mathrm{UBB}^{+1}$ accumulation 
causes mitochondrial damage. We identified the $\mathrm{UBB}^{+1}$-induced enhancement of the basic amino acids arginine, ornithine and lysine at mitochondria as a toxic event causing damage to the mitochondria. Hereby the protein cytochrome $c$ and other factors can be released which eventually will lead to cell death.

In parallel, we identified a mechanism which protects the cell against the harmful effects of $\mathrm{UBB}^{+1}$. A protein called Vms1 can relieve the $\mathrm{UBB}^{+1}$-triggered mitochondrial dysfunction and cell death. Our study proposed that Vms1-dependent mitochondrial quality control might retard the $A D$ associated neuronal dysfunction. We believe that $A D$-induced cellular dysfunctions can be avoided by UPS activity at mitochondria has far-reaching pathophysiological implications.

The present thesis (Chapters 5 and 6) also revealed another possibility to develop therapeutic applications. A decrease in A $\beta$ plaque load in APPPS1/UBB ${ }^{+1} \mathrm{tg}_{\text {mice is caused }}$ by rises in $\mathrm{Y}$-secretase activity/PS1 expression. In the current scientific paradigm it is thought that it is necessary to inhibit the $\mathrm{Y}$-secretase activity as PS1 is responsible for the cleavage of APP C-terminal fraction (APP-CTF) and thereby creates $A \beta$ which will accumulate in $A \beta$ plaque load. However, as mentioned above, until now treatments with $\gamma^{-}$ secretase inhibitors were so far unsuccessful (De Strooper, 2014). Therefore, based upon our results in the triple $\operatorname{tg}$ APPPS1/UBB ${ }^{+1}$ line, we suggest to examining the effects of activation of $\gamma$-secretase activity. We anticipate finding lower number of $A \beta$ plaques which possibly will have an effect on behavioural read-outs.

Both ideas mentioned above for possible therapeutic applications are molecular hypotheses which need more input. However, such research is still in its infancy, so both approaches are valuable options to investigate further and hopefully contribute to an amelioration or cure for AD. 


\section{References}

(Alzheimer's Association 2015). "2015 Alzheimer's disease facts and figures ", in: Alzheimer's \& Dementia.).

Attems, J., Walker, L., and Jellinger, K.A. (2014). Olfactory bulb involvement in neurodegenerative diseases. Acta Neuropathol 127, 459-475.

Barnes, D.E., and Yaffe, K. (2011). The projected effect of risk factor reduction on Alzheimer's disease prevalence. Lancet Neurology 10, 819-828.

De Jong, L.W., Ferrarini, L., Van Der Grond, J., Milles, J.R., Reiber, J.H., Westendorp, R.G., Bollen, E.L., Middelkoop, H.A., and Van Buchem, M.A. (2011). Shape abnormalities of the striatum in Alzheimer's disease. Journal of Alzheimer's Disease 23, 49-59.

De Strooper, B. (2014). Lessons from a failed gamma-secretase Alzheimer trial. Cell 159, 721-726.

Djordjevic, J., Jones-Gotman, M., De Sousa, K., and Chertkow, H. (2008). Olfaction in patients with mild cognitive impairment and Alzheimer's disease. Neurobiology of Aging 29, 693-706.

Drachman, D.A. (2014). The amyloid hypothesis, time to move on: Amyloid is the downstream result, not cause, of Alzheimer's disease. Alzheimers Dement 10, 372-380.

Geerlings, M.I., Den Heijer, T., Koudstaal, P.J., Hofman, A., and Breteler, M.M. (2008). History of depression, depressive symptoms, and medial temporal lobe atrophy and the risk of Alzheimer disease. Neurology 70, 1258-1264.

Holmes, C., Boche, D., Wilkinson, D., Yadegarfar, G., Hopkins, V., Bayer, A., Jones, R.W., Bullock, R., Love, S., Neal, J.W., Zotova, E., and Nicoll, J.A. (2008). Long-term effects of Abeta42 immunisation in Alzheimer's disease: follow-up of a randomised, placebo-controlled phase I trial. Lancet 372, 216-223.

Humbert, I.A., Mclaren, D.G., Kosmatka, K., Fitzgerald, M., Johnson, S., Porcaro, E., Kays, S., Umoh, E.O., and Robbins, J. (2010). Early deficits in cortical control of swallowing in Alzheimer's disease. J Alzheimers Dis 19, 1185-1197.

Igap (2015). International Genomics of Alzheimer's Disease Consortium. Convergent genetic and expression data implicate immunity in Alzheimer's disease. Alzheimer's \& Dementia 11, 658-671.

Lannfelt, L., Moller, C., Basun, H., Osswald, G., Sehlin, D., Satlin, A., Logovinsky, V., and Gellerfors, P. (2014). Perspectives on future Alzheimer therapies: amyloid-beta protofibrils - a new target for immunotherapy with BAN2401 in Alzheimer's disease. Alzheimers Res Ther 6, 16.

Manavalan, A., Mishra, M., Feng, L., Sze, S.K., Akatsu, H., and Heese, K. (2013). Brain site-specific proteome changes in aging-related dementia. Experimental \& Molecular Medicine 45, e39.

Mitchell, R.A., Herrmann, N., and Lanctot, K.L. (2011). The role of dopamine in symptoms and treatment of apathy in Alzheimer's disease. CNS Neuroscience \& Therapeutics 17, 411427.

Mobley, A.S., Rodriguez-Gil, D.J., Imamura, F., and Greer, C.A. (2014). Aging in the olfactory system. Trends Neurosci 37, 77-84.

Sierksma, A.S., Van Den Hove, D.L., Steinbusch, H.W., and Prickaerts, J. (2010). Major depression, cognitive dysfunction and Alzheimer's disease: is there a link? European Journal of Pharmacology 626, 72-82. 
Sinha, U.K., Hollen, K.M., Rodriguez, R., and Miller, C.A. (1993). Auditory system degeneration in Alzheimer's disease. Neurology 43, 779-785.

Wilson, R.S., Mendes De Leon, C.F., Bennett, D.A., Bienias, J.L., and Evans, D.A. (2004). Depressive symptoms and cognitive decline in a community population of older persons. Journal of Neurology, Neurosurgery, and Psychiatry 75, 126-129. 


\section{List of abbreviations}


IV

5-HT

$10 \mathrm{~N}$

$12 \mathrm{~N}$

$A \beta$

$A B C$

$A D$

AICD

Aif1

$\mathrm{aMCl}$

AP

$\mathrm{APH}-1$

APOE

APP

APP-CTF

APP-FL

APPS

Arg

CA

CAD

CamKIla

CFU

CTF

Cytc

D

DAB

DAergic

DG

DHE

DS

DUB fourth ventricle

serotonin

dorsal motor nucleus of the vagus nerve

hypoglossal nucleus

amyloid $\beta$

avidin-biotin complex

Alzheimer's disease

APP intracellular domain

apoptosis-inducing factor 1

amnestic mild cognitive impairment

area postrema

ATP-dependent proteolytic factor 1

apolipoprotein E

amyloid precursor protein

amyloid precursor protein C-terminal fragment

amyloid precursor protein full-length

soluble N-terminal part of APP

arginine

cornu ammonis

central auditory dysfunction

calmodulin kinase Ila

colony forming units

C-terminal fraction

cytochrome c

density

3,3'-diaminobenzidine tetrahydrochloride

dopaminergic

dentate gyrus

dihydroethidium

Down syndrome

deubiquitinating enzyme 
ECL

EEG

EF1a

EGFR

EOAD

ER

ERAD

FAD

FC

FENIB

FITC

G

GAMPO

GAPDH

GARPO

GFAP

GFP

GWAS

HD

HECT

HRD1

Hsp25

HTh

I

IC

IDE

IGAP

IOD

ir

IRt

JAMM enhanced chemiluminescence

electroencephalography

elongation factor 1-alpha 1

epidermal growth factor receptor

early-onset $A D$

endoplasmatic reticulum

endoplasmic reticulum associated degradation

familial Alzheimer's disease

fear conditioning

familial encephalopathy with neuroserpin inclusion bodies

fluorescein isothiocyanate

glycine

goat anti-mouse horseradish peroxidase-conjugated antibody

glyceraldehyde 3-phosphate dehydrogenase

goat anti-rabbit horseradish peroxidase-conjugated antibody

glial fibrillary acidic protein

green fluorescent protein

genome wide association studies

Huntington's disease

homologous to E6-associated protein C-terminus

HMG-CoA reductase degradation 1

heat shock protein 25

hypothalamus

intensity

inferior colliculus

insulin degrading enzyme

International Genomics of Alzheimer's disease Project

integrated optical density

immunoreactivity

intermediate reticular tract

JAB1/MPN/Mov34 metalloenzyme 
K

॥

lysine

LC

lateral lemniscus

locus coeruleus

LOAD

late-onset $A D$

LRt

lateral reticular nucleus

LRtS5

subtrigeminal part of the lateral reticular nucleus

Lys

lysine

$\mathrm{MCl}$

mild cognitive impairment

MJD

Machado-Joseph Disease protein domain protease

MRI magnetic resonance imaging

MWM

Morris water maze

NA

noradrenaline

NBM

nucleus basalis of Meynert

NFkB

nuclear factor $\mathrm{k} \beta$

NFT

neurofibrillary tangle

NMD

nonsense-mediated RNA decay

NMDA

$\mathrm{N}$-methyl-D-aspartate

NP

neuritic plaque

NPY

neuropeptide $Y$

NSC

neural stem cell

NT

neuropil thread

NTS

nucleus of the solitary tract

Nuc1

endonuclease G

OB

olfactory bulb

OD

optical density

OTU

ovarian tumor proteases

PBN

parabrachial nucleus

PBNcau

parabrachial nucleus, caudal part

$\mathrm{PBNCl}$

parabrachial nucleus, central lateral part

PBNdl

parabrachial nucleus, dorsal lateral part

PBNel

parabrachial nucleus, external lateral part 
PBNem

PBNI

PBNm

PBNvl

PBS

PD

PEI

Pen2

PHF

PI

PQC

PS1

PS1-FL

PS1-NTF

PS2

RDD

RER1

RFU

RING

Rip1

RLU

ROS

Rpt

Rs27a

RT

SAD

SAG

scp

SILAC

sol

SolDL parabrachial nucleus, external medial part

parabrachial nucleus, lateral part

parabrachial nucleus, medial part

parabrachial nucleus, ventrolateral part

phosphate-buffered saline

Parkinson's disease

polyethyleneimine

presenilin enhancer 2

paired helical filament

propidium iodide

protein quality control

presenilin 1

presenilin 1 full-length

presenilin $1 \mathrm{~N}$-terminal fragment

presenilin 2

RNA-DNA sequence differences

retention in endoplasmic reticulum sorting receptor 1

relative fluorescence units

real interesting new gene

Rieske iron-sulfur protein

relative luminescence unit

reactive oxygen species

regulatory particle triple

ubiquitin-40S ribosomal protein S27a

room temperature

sporadic Alzheimer's disease

sagulum

superior cerebellar peduncle

stable isotope labeling by amino acids in cell culture

solitary tract

nucleus of the solitary tract, dorsolateral part 
SolIM

SolM

SPECT

Sp5

SPP

SWD

TBS

TDP-43

$\operatorname{tg}$

Ti/TT

TMRM

TREM2

TUNEL

UBB

$\mathrm{UBB}^{+1}$

$\mathrm{UBB}^{+1}$ |

$\mathrm{UBB}^{+1} \mathrm{D}$

UBC

$\mathrm{UCH}$

UCHL-1

UFD

UPR

UPS

USP

USP14

VDAC1

VMS1

WT, wt

Ybh3 nucleus of the solitary tract, intermediate part

nucleus of the solitary tract, medial part

single-photon emission computed tomography

spinal 5 nucleus

subpeduncular pigmented nucleus

sharp wave discharges

Tris-buffered saline

transactive response DNA binding protein 43

transgenic

relative duration of inspiration

tetramethylrhodamine methyl ester

triggering receptor expressed on myeloid cells 2

terminal deoxynucleotidyl transferase dUTP nick end labeling

ubiquitin $B$

ubiquitin $\mathrm{B}^{+1}$

intensity of $\mathrm{UBB}^{+1}$ immunoreactivity

density of $\mathrm{UBB}^{+1}$ immunoreacitivity

ubiquitin $\mathrm{C}$

ubiquitin C-terminal hydrolase

ubiquitin carboxy-terminal hydrolase isozyme L1

ubiquitin fusion degradation

unfolded protein response

ubiquitin proteasome system

ubiquitin specific proteases

ubiquitin specific peptidase 14

voltage-dependent anion channel 1

VCP/Cdc48-associated mitochondrial stress-responsive

wild type

yeast $\mathrm{BH} 3$-only protein 
Publication list 


\section{Scientific publications in international peer-reviewed journals}

a. R.J. Gentier and F.W. van Leeuwen. Misframed ubiquitin and lack of protein quality control; an early event in Alzheimer's disease. Frontiers in Molecular Neuroscience.2015;8:1-12

b. R.J. Gentier, B.M. Verheijen, M. Zamboni, M.M. Stroeken, D.J.H.P. Hermes, B. Küsters, H.W.M. Steinbusch, D.A. Hopkins, F.W. Van Leeuwen. Localization of mutant ubiquitin in the brain of transgenic mouse line with proteasomal inhibition and its validation at specific sites in Alzheimer's disease, Frontiers in Neuroanatomy.2015;9(26):1-21.

c. M. Irmler*, R.J. Gentier*, F.J. Dennissen, H. Schulz, I. Bolle, S.M. Hölter, M. Kallnik, J.J. Cheng, M. Klingenspor, J. Rozman, N. Ehrhardt, D.J.H.P. Hermes, V. Gailus-Durner, H. Fuchs, M. Hrabě de Angelis, H.E. Meyer, D.A. Hopkins, F.W. Van Leeuwen, J. Beckers. Long-term proteasomal inhibition in transgenic mice by UBB+1 expression results in dysfunction of central respiration control reminiscent of brainstem neuropathology in Alzheimer patients, Acta Neuropathologica.2012; 124(2):187-197. * Co-first author

d. R.J. Braun*, C. Sommer*, C. Leibiger*, R.J. Gentier*, V.I. Dumit, K. Paduch, T. Eisenberg, L. Habernig, G. Trausinger, C. Magnes, T. Pieber, F. Sinner, J. Dengjel, F.W. Van Leeuwen, G. Kroemer, F. Madeo. Accumulation of basic amino acids at mitochondria dictates the cytotoxicity of aberrant ubiquitin, Cell Reports.2015; 10(9):1557-1571. * Co-first author

e. P. van Tijn*, F.J. Dennissen*, R.J. Gentier, B. Hobo, D.J.H.P. Hermes, H.W.M. Steinbusch, F.W. Van Leeuwen. Mutant ubiquitin transiently decreases amyloid $\beta$ plaque formation in a transgenic mouse model of Alzheimer's disease, Neurochemistry International.2012;61(5):739-748.* contributed equally. 


\section{To be submitted}

f. R.J. Gentier, J.Stevens, L.M. Verheijen, C.D. van 't Hekke, D. van den Hove, D.H.J.P. Hermes, H.W.M. Steinbusch, J.J. Cheng, M.O. Grimm, V. J. Haupenthal, W. Annaert, T. Hartmann and F.W. van Leeuwen. Paradoxical effects of mutant ubiquitin and $y$-secretase during modelled Alzheimer's disease neuropathogenesis. in preparation 



\section{Curriculum Vitae}


Romina Gentier werd geboren op 6 juli 1987 in Bilzen, België. In 2005 behaalde ze haar diploma secundair onderwijs, Latijn-Wiskunde aan het Heilig-Graf Instituut te Bilzen. Hierna begon ze haar universitaire opleiding Biomedische Wetenschappen aan de Universiteit Hasselt. In 2008 behaalde ze haar bachelor diploma en vervolgens begon zij aan haar master Clinical and Molecular Life Sciences. Ze deed haar junior en senior stage aan de School for Mental Health and Neurosciences (MHeNS), divisie Neurosciences onder de supervisie van Dr. Fred van Leeuwen. In 2010 behaalde ze haar masterdiploma met vermelding grote onderscheiding. Aansluitend startte ze haar promotie-onderzoek bij MHeNS aan de Universiteit Maastricht onder begeleiding van Dr. Fred van Leeuwen als co-promotor en Prof. Dr. Harry Steinbusch en Prof. Dr. David Hopkins als promotoren. Het uitgevoerde onderzoek over $\mathrm{UBB}^{+1}$ staat beschreven in dit proefschrift.

Romina Gentier was born on July 61987 in Bilzen, Belgium. In 2005, she graduated from high school where she followed Latin-Mathematics at Heilig-Graf Institute in Bilzen. Thereafter, she started to study Biomedical Sciences at the University of Hasselt. In 2008, she received her bachelor's degree and in the same year she started her master in Clinical and Molecular Life Sciences. She performed her junior and senior internship at the School for Mental Health and Neurosciences (MHeNS), a school of Maastricht University under the supervision of Dr. Fred van Leeuwen. In 2010, she graduated and she received her master's degree with the mention great honors. Subsequently, she started her PhD trajectory at MHeNS with Dr. Fred van Leeuwen as co-promotor and Prof. Dr. Harry Steinbusch and Prof. Dr. David Hopkins as promotors. The research performed about the $\mathrm{UBB}^{+1}$ protein during the 4-year $\mathrm{PhD}$ is described in this thesis. 


\section{Acknowledgements}

\section{Dankwoord}


Het dankwoord, de paragraaf die volgens mij het meest gelezen wordt in een PhD thesis. Toegegeven dit is ook één van de eerste paragrafen die ik zelf altijd gelezen heb in een thesis. Nu ben ik degene die hieraan toe is om dit deel te schrijven en af en toe leek dit deel een ver-van-mijn-bed-show. Maar mijn dankwoord is er en ik ben nu blij dat veel mensen dit zullen lezen. Want deze thesis en alles daaromheen was nooit mogelijk geweest zonder de hulp en kennis van heel wat mensen. Deze verdienen dan ook een oprechte dankjewel in dit boekje.

Allereerst wil ik Prof. Dr. Harry Steinbusch bedanken om me de kans te geven een PhD te doen binnen MHeNS en me bij te staan bij het schrijven van deze thesis. Vervolgens Fred, mijn supervisor, je hebt me veel geleerd en ik ben enorm blij dat ik je laatste PhD-student mocht zijn. Altijd stond je deur open en binnenlopen mocht en deed ik dan ook wanneer het mezelf uitkwam. Je hebt me de kans gegeven om naar Society for Neuroscience te gaan en dat was een super ervaring. Er was gedurende die 5 jaar heel wat wetenschappelijk overleg, vaak werd er gelachen, soms gediscussieerd. Maar bovenal onthoud ik dat je naast een zeer goede wetenschapper ook een goed mens bent die respect heeft voor mens en omgeving. Bedankt Fred!

David Hopkins, onze Canadese visiting-professor. Ik leerde je kennen tijdens mijn master stage bij Fred en toen had je me al belangeloos geholpen met mijn master thesis. Elk jaar kwam je terug en je hebt een zeer grote bijdrage gedaan aan deze PhD thesis. Aan jou anatomisch inzicht kan niemand tippen en het is echt iets waar ik stiekem een beetje jaloers op ben. Maar ik durf met trots te zeggen: David, je hebt me echt heel wat over de anatomie van de hersenen bijgebracht. Daarnaast ben je regelmatig met je rode pen druk aan het werk geweest om de eerste versies van de artikels te verbeteren. David, thanks for everything!

Denise en Marjan, mijn paranimfen en onze superanalysisten. Ik heb heel vaak gedacht hoe blij ik wel niet was dat jullie er waren op het lab. Altijd mocht ik jullie storen met mijn vragen en jullie hielpen me altijd met een grote lach. Ik heb doorheen de jaren alleen maar meer respect gekregen voor jullie kennis en kunde en vooral voor jullie geduld :). Ook een leuke babbel of eens klagen over bepaalde situaties stonden regelmatig op ons programma. Ze mogen in Maastricht blij zijn met analysisten zoals jullie. Denise en Marjan, 
bedankt om mijn bij te staan bij mijn PhD promotie, bedankt voor jullie praktische hulp en voor jullie vriendschap.

Hellen, bedankt dat je me zeer regelmatig bijgestaan hebt met raad en daad over immunohistochemische kleuringen en het snijden van weefsel. Ook wil ik hier een oprechte "Dank je wel“ zeggen tegen Veerle Baert, analysist aan het VIB, Center for the Biology of Disease. Je hebt mij en mijn studenten enorm goed geholpen met het Western Blot verhaal. Als er iemand een professional is in Western Blot ben jij het wel :). De werkdagen in Leuven waren super leuk. Je was er altijd voor een toffe Vlaamse babbel. Even wist ik zeker dat als ik iets zei in het Vlaams dat ik ook gewoon begrepen werd :).

Daniel van den Hove, ook jou moet ik oprecht bedanken. Opeens werd het analyseren van gedragsdata een deel van mijn thesis en als leek was dit niet zo simpel voor mij. Je hebt me dan ook spontaan geholpen met de analyse en de interpretatie van deze data. Also Ralf Braun of the University of Bayreuth, thanks for giving me the opportunity to work together with you and your team in the yeast-story. I really enjoyed working together with you at the immunohistochemical lab, making long days to reach good results. Very recently, I was a guest at your "Yeast-group" at the University of Bayreuth. I had a wonderful week with you and your friendly colleagues. I really appreciate your hospitality and my mother and Artuur enjoyed the beautiful city of Bayreuth. Thanks for everything!

Prof. Dr. Wim Annaert van de VIB, Center for the Biology of Disease, enorm bedankt om me de kans te geven om onze Western Blot experimenten uit te voeren in jouw lab en voor de leerrijke gesprekken die we op regelmatige basis gevoerd hebben. Ik heb veel geleerd en genoten van mijn tijd in jullie lab.

Also thanks to the group of Martin Irmler and the German Mouse Clinic in Munchen. You performed an amazing screening of our $\mathrm{UBB}^{+1}$ transgenic mice and we could perfectly combine your data with our immunohistochemical stainings in these mice and in human brain tissue. We published together a nice article in ANP. Thanks for working together in such a nice and friendly way.

I am also grateful to the group of Prof. Dr. Tobias Hartmann and Dr. Markus Grimm because they performed a lot of secretase measurements in our mice. 
Een PhD is een hele ontdekkingsreis die voor mij begonnen is op 1 september 2010. Als braaf Belgisch meisje daar in Nederland gaan werken tussen de volmondige Nederlanders :). Wat was ik enorm zenuwachtig! Maar ik was dan ook zo blij dat jij, Ine Rayen, op dezelfde dag als mij begon aan je PhD. Ik ken je al van $1^{\mathrm{e}}$ bachelor jaar Biomedische wetenschappen aan de Universiteit Hasselt. Daar zaten we al van dag 1 in dezelfde groep en we hebben samen ons PhD traject doorlopen in Maastricht. Bedankt voor de leuke gesprekken, de gezellige lunches en het helpen met allerlei zaken. Ondertussen spreken we heel regelmatig af voor een etentje met onze kindjes en dit is altijd super gezellig! Nog iemand die heel veel voor me gedaan heeft is Ramona Hohen. Je zorgde altijd voor leuke lunches, je stond klaar om naar mijn geklaag te luisteren en gaf me dan ook goede raad. ledereen weet dat je de beste kokkin van de afdeling bent en je deelde vaak je lekkernijen. Af en toe ontsnapten we even naar de UNS60 en even genieten van een wandeling of een ijsje. Buiten dit heb je me ook altijd geholpen met mijn praktische vragen. Bedankt daarvoor! Ook Elke Kuypers bedankt voor de ontspannende gesprekjes tussendoor op het lab. Ook jou ken ik al van het $1^{\mathrm{e}}$ bachelor jaar aan de UHasselt. Wat was het af en toe toch even leuk om tegen iemand meer vertrouwd een zegje te kunnen doen. Ondertussen zijn onze zoontjes Emiel en jouw Seppe ook vriendjes geworden :). Liesbeth Knaepen, ook jij zorgde voor een aangename lunchtijd en bij warm weer gezellig buiten. De leuke babbels hielpen om weer even te ontspannen alvorens weer verder te werken. Veel succes met je gezinnetje :).

Ook Jo Stevens, een andere mede-Belg onder de Nederlanders :). Bedankt voor de leuke babbels en voor je wijsheid. Als ik iets niet wist, was vaak het antwoord: „Vraag het eens aan, Jo“. Bedankt voor je hulp bij het gedrag en het Western Blot verhaal. Frank Dennissen, mijn kamergenoot en PhD-collega binnen ons klein $\mathrm{UBB}^{+1}$ groepje. Bedankt voor de leuke tijd in onze kamer, de interessante babbels over de toch wel aanwezige verschillen tussen Belgen en Nederlanders :) en het delen van je kennis en inzicht over het UPS systeem. Also Sarah Hescham thanks for the great time at Society for Neuroscience 2012 in New Orleans. You were a great roommate sharing stories and experiences. Also special thanks to Yuan Tian, my Chinese roommate. I really liked my talks with you about China, traveling, etc. You were always very interested in Emiel and Artuur. Thanks for the great time together and I wish you luck with your new projects in life. Also Anna, my last roommate at the 
UNS40, thanks for the great time together and our talks about children and their problems. Wish you the best for you, Ben and your family. Ook Jochen de Vry en Caroline Hammels, weer mede-Belgjes, bedankt voor de toffe babbels en veel geluk met jullie kleine Lars. Nog andere mensen die zorgden voor een aangename sfeer op de werkvloer: Marlien, Annerieke, Marlies, Jodi, Nicole, Sandra, Nienke, Joao, Ehsan, Julie, Sven, Nick, Celine en alle andere collega's van departement Neurosciences.

Deze thesis zou er ook niet geweest zijn door de hulp van verschillende Bachelor en Master studenten die hun Major, Junior of Senior stage volgden bij ons. Elk heeft op zijn manier een bijdrage geleverd aan deze thesis. Aron, Bart, Lara, Maartje, Margherita, Mark en Stephanie bedankt voor jullie hulp en ik vond het heel leuk om jullie te begeleiden tijdens jullie stage.

Vervolgens zijn we aangekomen bij het thuisfront. Jullie zijn allemaal belangrijk geweest in de mentale ondersteuning van dit project door te zorgen voor de nodige ontspanning of door het bieden van een luisterend oor. Zonder vrienden kan een mens geen geluk vinden en laat het nu net mijn vrienden zijn die heel belangrijk zijn voor mij.

Sabine, mijn beste dinneke en buurmeisje al vanaf ons $10^{\mathrm{e}}$ levensjaar. Ik kan al niet meer tellen hoe vaak we er al met ons twee op uit getrokken zijn: festivals, concerten, sightseeing en vooral "people watching" :), terrasjes, cinemake, shoppen, af en toe een vakantietje tussendoor en als het geen van deze is dan is het gewoon lui in de zetel hangen en brainless tv kijken. Maar al deze dingen hebben ervoor gezorgd dat ik mij naar behoren kon ontspannen en dat maakt natuurlijk een gelukkiger mens. Bieni, bedankt om er te zijn.

Wim oftewel Wimpie, ook jij behoort bij mijn beste vrienden en bent er altijd geweest wanneer dit nodig was. Voor leuke momenten maar evenzeer ook eens voor minder leuke momenten. Ik apprecieer enorm dat ik zo goed met je kan babbelen en ik kan je altijd in vetrouwen nemen. Ook stond je altijd op de eerste rij om mee de wijde wereld in te trekken. Bieni en Wimpie jullie zijn niet voor niets de meter en de peter van onze lieve Emiel. Bedankt voor alles! PS: die uitstappen en dergelijke gaan in de toekomst gewoon verder samen met Brenda en Gustavo! 
De andere leden van onze "Bilzen clan" en dan heb ik het over: Frank en Dorien, Wout en Lisa, Jan en Ine, Michiel en Carolin, Jordan, Stijn, Sofie en Kim. We kennen elkaar allemaal al behoorlijk lang en het is fijn om zo een toffe vriendengroep te hebben waar we regelmatig eens iets mee gaan drinken, een concertje of een filmpje mee pikken. Een leuke gevarieerde groep waar iedereen zichzelf kan en mag zijn. Bedankt voor jullie steun en vriendschap doorheen de jaren.

Ook Dorien, An-Sofie en Evy+Jeffrey, vriendinnetjes van de lagere school en het middelbaar. Jullie waren en zijn er ook altijd als ik jullie nodig heb. Toffe uitstapjes en gezellige etentjes maken het leven weer een stuk aangenamer. Dank jullie wel!

Ook voor Joris is er een speciaal plaatsje gereserveerd in dit dankwoord. In 2005 zijn we beiden begonnen aan de opleiding Biomedische Wetenschappen aan de UHasselt, eigenlijk toen nog het LUC. Ik startte dag 1 zenuwachtig en zonder ook maar iemand te kennen. In de aula ging ik dan ook maar lukraak ergens zitten. Dit was toevallig naast jou en toen wist ik nog niet dat je een hele goede vriend zou worden. Doorheen de bachelor hebben we heel wat toffe momenten beleefd: people watching op de Agora, lessen skippen en dan maar naar de Quick wandelen of toch ook maar eens studeren in park van Hasselt. Een belangrijk ding heb ik geleerd, wij samen in het lab tijdens pratica was een ramp: dampende mengels, ninhydrine gebruiken buiten de afzuigkast en tritreren met het hele flesje in plaats van 3 druppels. Na de bachelor heb je beslist om aan de Universiteit Maastricht geneeskunde te gaan studeren en ik vond het zo erg dat ik nu zonder jou aan de Master moest beginnen. Gelukkig kwam ik je nog heel regelmatig tegen in Maastricht tijdens mijn PhD. Vandaag verdedig ik mijn PhD en jij bent bezig aan je specialisatie als arts Internist-Intensivist. Dus Joris, slecht hebben we het er niet van afgebracht toch :). Bedankt voor alle bijzondere momenten en ik wens je alle geluk toe in je opleiding en in je prive-leven met Pieter-Jan.

Verder zijn er nog verschillende vrienden die oprecht een plaatsje krijgen in dit boekje: Karen en Thomas, Laura en Dennis, Will en Sara, Leen en Lore. Bedankt voor de steun en de gezellige avonden. Catherine en Gert-Jan, verschillende gezellige avonden hebben me geleerd dat ik niet alleen ben met de kleine zorgen van een PhD student. Catherine, Artuur heeft een super meter gekozen :) en veel geluk met jullie gezinnetje! 
Een ander heel belangrijk deel van mijn leven is mijn familie die me altijd gesteund hebben in de zaken die ik wilde bereiken in mijn leven. Daarom dank je wel aan mijn grootouders, mijn nichten en neven (Tessie, Silke, Jeffrey, Dorothy, Joshua, Lindy, Lindsay, Liesbeth en Michael), mijn tantes (Colette, Lut, Grit, Carine Hoeselt en Carine Munsterbilzen), en nonkels (Jan Munsterbilzen (2x), Lode, en Jean), alsook schoonbroer en schoonzusje en partners Tony en Sim, Christina en Glenn. Ook mijn broer Berny en zijn vriendin Wendy, bedankt om er te zijn voor mij als ik jullie nodig heb. Hoe verschillend onze karakters ook zijn Berny, uiteindelijk zijn we er voor elkaar als het nodig is en dit is de belangrijkste eigenschap in het leven. Artuur heeft een goede peter aan je gevonden.

Ook Marleen en Patrick, mijn schoonmama en schoonpapa, bedankt voor jullie vertrouwen in mijn kunnen en voor het helpen om alles georganiseerd te krijgen met de kindjes. Ze kunnen geen betere Oma en Opa Koekentaart wensen :).

Dan nog iemand die voor mij een van de belangrijkste personen in mijn leven is; mama. Mama, ik kan me geen betere mama inbeelden. Jij bent degene die me in elke stap onvoorwaardelijk gesteund heeft en vertrouwde in mijn kunnen. Ik ben je dan ook uiterst dankbaar voor alle kansen die je me al van kindsbeen af hebt gegeven en die me mee gevormd hebben tot de persoon die ik ben. Naast een supermama ben je ook een super Omi.

Ook een dank je wel aan mijn hond Louis. Ook al spreek je niet, je kwam altijd bij me liggen als ik me door moeilijke artikels worstelde of als ik moeilijke teksten aan het schrijven was. Bedankt voor je warmte en vertrouwen.

En mijn dankwoord afsluiten wil ik doen met de personen die ik onvoorwaardelijk graag zie. Niko, al 12 jaar zijn we samen en al 12 jaar delen we dan ook lief en leed. Jij was degene die altijd zei dat ik zeker de capaciteit had om universitaire studies te doen, om een PhD te doen, om avondschool te volgen en noem maar op. Bedankt om blindelings je vertrouwen in mij te leggen. Je hebt me niet alleen mentaal maar ook praktisch enorm geholpen bij dit project: de kaft, de figuren, de lay-out van het boekje... Bedankt Giraffie ;). Samen hebben we 2 prachtige kinderen: Emiel en Artuur en dit is het mooiste wat je me hebt kunnen geven. 
Emiel en Artuur, mijn 2 prachtige zoontjes, jullie zijn het beste dat me ooit kon overkomen en jullie hebben me geleerd wat bovennatuurlijke liefde betekent. Jullie toveren elke dag een glimlach op mijn gezicht en ik ben dan ook trots dat ik jullie mama mag zijn. Ik hou van jullie!!! 